\title{
Effects of Atom-Laser Interaction on Ultra-Cold Atoms
}

\author{
Dissertation \\ zur Erlangung des Doktorgrades \\ der Mathematisch-Naturwissenschaftlichen Fakultäten \\ der Georg-August-Universität zu Göttingen \\ vorgelegt von \\ Volker Martin Hannstein \\ aus Freiburg
}

Göttingen 2006 
D7

Referent: Prof. Dr. G. C. Hegerfeldt

Korreferent: Prof. Dr. K. Schönhammer

Tag der mündlichen Prüfung: 03.04.2006 


\section{Contents}

1 Introduction 3

I Dipole-dipole interacting atoms in a trap 5

2 Quantum jumps in the fluorescence of single atoms 10

2.1 The quantum jump approach . . . . . . . . . . . . . . 10

2.1.1 The conditional Hamiltonian . . . . . . . . . . . . . . . . . 10

2.1 .2 The reset state . . . . . . . . . . . . . . . . 12

2.1.3 The optical Bloch equation . . . . . . . . . . . . . . . . 13

2.1.4 Application to three-level systems . . . . . . . . . . . . . . . 14

2.2 Description of the fluorescence properties . . . . . . . . . . . . . . 16

2.3 Experimentally relevant level structures . . . . . . . . . . . . . . . . 18

2.3.1 The D system . . . . . . . . . . . . . . . 19

2.3.2 The four-level system for the description of the $\mathrm{Ba}^{+}$ions . . . 19

3 Cooperative quantum jumps for two and three dipole-interacting $\begin{array}{ll}\text { atoms } & 21\end{array}$

3.1 Quantum jump approach for dipole-interacting atoms . . . . . . . . . 22

3.1.1 The conditional Hamiltonian for dipole-interacting three-level systems . . . . . . . . . . . . . . . . . . . 23

3.1 .2 The reset state . . . . . . . . . . . . . . . 25

3.2 Two dipole-interacting atoms . . . . . . . . . . . . . 25

3.2.1 Two V systems . . . . . . . . . . . . . . . . . 26

3.2 .2 Two D systems . . . . . . . . . . . . . . . . . . . 33

3.2.3 Two four-level systems as a model for two $\mathrm{Ba}^{+}$-ions . . . . . . 37

3.3 Three dipole-interacting atoms . . . . . . . . . . . . . . . . . . . 42

3.3.1 Conditional Hamiltonian and reset state . . . . . . . . . . . . 42

3.3.2 Group theoretical methods . . . . . . . . . . . . . . . . . 44

3.3 .3 Transition rates . . . . . . . . . . . . . . . . . . 47

3.3.4 Double and triple jump rate . . . . . . . . . . . . 50

3.3.5 Simplified approach: Treatment of three four-level systems . . 53 
II Detection of ultra-cold moving atoms

4 Atoms in evanescent laser fields 64

4.1 Description of the experiment . . . . . . . . . . . . . . 64

4.2 Evanescent wave atomic mirrors . . . . . . . . . . . . . . . 67

4.2 .1 The dipole force . . . . . . . . . . . . . . . . . . 68

4.2 .2 Atoms in exponential potentials . . . . . . . . . . . . 71

4.3 Arrival-times in quantum mechanics . . . . . . . . . . . . . . 73

4.3 .1 Classical arrival-time . . . . . . . . . . . . . . . . 73

4.3.2 Quantum arrival-times ... . . . . . . . . . . 75

5 The atomic mirror as a measuring device 78

5.1 Calculation of the reflected norm . . . . . . . . . 78

5.1.1 Momentum representation point of view . . . . . . . . 79

5.1 .2 Green's function point of view . . . . . . . . . . . . 83

5.2 Behavior for short switch-on times of the mirror . . . . . . . 85

5.3 Numerical analysis of the model . . . . . . . . . . . . . . . . . 91

5.3.1 Measurement of the spatial distribution of the wave function . 93

5.3.2 Measurement of temporal distributions . . . . . . . . . . 97

5.3.3 Reflected and transmitted wave packet in position space . . . 100

5.4 3D Model: Tomography of the wave packet . . . . . . . . . . . 103

6 Conclusion 107

A Quantum jump approach for two D systems 112

B Exact transition rates for three four-level systems 114

$\begin{array}{ll}\text { C Hilbert transforms } & 115\end{array}$ 


\section{Chapter 1}

\section{Introduction}

The control and manipulation of the atomic translational degrees of freedom via atom-laser interaction is an essential tool in modern quantum optical experiments. The underlying basic mechanism for this is the transfer of the photon's momentum when it is absorbed or emitted by the atom. The sophisticated implementation of these basic processes allows for a large variety of applications.

For example it has become possible in the last decades to slow down the atomic motion to velocities of the order of centimeters or even millimeters per second. These cooling techniques allow to trap atoms or ions in suitable laser fields or radiofrequency electromagnetic fields, respectively. The atoms in these traps can be cooled down to their motional ground state and can be stored for practically arbitrarily long times. Atom traps permit many interesting experiments concerning the foundations of quantum mechanics and allowed the realization of rudimentary quantum computers. Today they are one of the most promising candidates for the development of quantum computers of practical use.

Another application of atom-laser interaction is atom optics. The goal of this area of research is to make the basic devices known from classical optics available for atoms and molecules. This has led to experimental realizations of atomic mirrors, lenses, interferometers, and even waveguides. Again, foundational aspects as well as very promising technical applications are the motivation for this field. Examples for the latter are atom lithography, in which atomic interference patterns are deposited on a substrate in order to create a periodic structure, or integrated atom optics devices on a chip for quantum information processing.

The present work is concerned with theoretical investigations which may shed some light on certain experiments in this field.

In the first part of this work cooperative effects in the fluorescence of dipole-dipole interacting atoms in a trap are studied. When illuminated by lasers, single trapped atoms which possess a metastable state in their effective level scheme, may, under certain conditions, exhibit macroscopic dark periods in their fluorescence signal. The spontaneous emission abruptly ceases for a certain period of time. In a simplified picture, this can be explained as a shelving of the electron in the metastable state. If 
two or three atoms showing this effect are stored simultaneously in a trap this leads to three or four periods of different intensity, namely a dark period and periods with once, twice or three times the intensity of a single atom. The statistics of these periods is influenced by the dipole-dipole interaction between the atoms in a sensitive manner. This work is concerned with a theoretical description of these effects, including a discussion of the seemingly contradictory experimental results which have been obtained in this context. The main result of this section indicates that the huge rate of double and triple jumps reported for three trapped $\mathrm{Ba}^{+}$ions can in fact not be attributed to the dipole-dipole interaction.

In the second part, the possible use of an evanescent wave atomic mirror as a measuring device for the properties of an atomic wave packet is investigated. The idea is taken from an experiment, in which such a mirror was used for the temporal diffraction of a cloud of laser cooled atoms dropping out of a magneto-optical trap. This was achieved by letting the atoms bounce off the mirror twice where each time the mirror was switched on for only a short time span. The switch-on time of the mirror can thereby be seen as the analogue of the width of a slit in the usual spatial diffraction experiments. The resulting distribution was analyzed by a third bounce and subsequent determination of the number of reflected atoms by a fluorescence measurement. In this work, the focus is set on the measurement procedure whereas the first two bounces are merely regarded as a way to prepare a sufficiently broad wave packet and are not further analyzed. A model for the investigation of the atomic wave function with such a mirror is given, and the outcomes of different setups are investigated. The main result of this section shows that with such a mirror one can sample the mod-squared of a spread-out wave function, and this opens the way for a realistic possibility for measuring this otherwise not easily accessible quantity.

More detailed introductions into the subjects of this work as well as outlines will be given at the beginning of each of the two parts. 


\section{Part I}

\section{Dipole-dipole interacting atoms in a trap}





\section{Introduction}

With the development of atom and ion traps it has become possible to store single atoms or ions for practically arbitrarily long times at temperatures in the sub-Kelvin regime. This allows to perform experiments, which give new insight into the foundations of quantum mechanics, and to observe effects which can not be seen in ensembles of particles.

One such effect is the occurrence of so-called macroscopic quantum jumps or macroscopic bright and dark periods in the spontaneous fluorescence of single atoms which are subject to laser radiation. The idea to this goes back to Dehmelt who, in 1975, proposed the following scheme in the context of high-precision spectroscopy [1]. Consider a three level atom in the so-called V configuration. The ground state of this atom is strongly coupled by a laser to an excited level. A second laser weakly couples the ground state to a third meta-stable level. The excitation by the strong laser leads to the emission of photons at a constant rate, typically of the order of $10^{8}$ photons per second. In a simplified picture, the weak laser pumps the electron to the meta-stable state where it is shelved for a certain period of time. This causes an intermission of the fluorescence signal until the electron returns to the ground state. About a decade after Dehmelt's proposal such macroscopic quantum jumps were indeed observed in experiments with single trapped $\mathrm{Ba}^{+}$ions $[2,3]$ and $\mathrm{Hg}^{+}$ions [4]. First theoretical work on this topic for incoherent [5] and coherent $[6,7,8,9,10]$ excitation showed that the fluorescence of such systems can be viewed as a two step telegraph process with exponentially distributed lengths of the different phases. Further research eventually led to the development of the quantum jump approach by Hegerfeldt and Wilser $[11,12,13,14]$ and the similar methods of quantum trajectories by Carmicheal [15] and the Monte Carlo wave function approach by Dalibard, Castin, and Mølmer [16].

The quantum jump approach describes the time development of a single quantum system interacting with the free radiation field and additional laser fields. It is well suited both for analytical and numerical analysis of such systems and provides a profound understanding of the effect described above.

Apart from single atoms or ions, it is also interesting to store small numbers of atoms in traps. In this case one has to take into account the dipole-dipole interaction between the atoms which may lead to cooperative effects in their radiative behavior. This topic is of great importance in many fields, most recently in the study of possible quantum computers based on trapped ions or atoms, and has therefore 
attracted considerable interest in the literature (see [17, 18] and references therein).

If there are two atoms in the trap, each exhibiting macroscopic quantum jumps, one expects three periods of different intensity in the overall fluorescence signal: a dark period, a period with the intensity of a single atom's bright period, and a period with twice this intensity. Accordingly, four different periods are to be expected for three atoms and so on. In addition to the usual jumps between periods whose intensities differ by once the intensity of a single atom there is also the possibility of multiple jumps. These occur if two or more consecutive upward or downward jumps happen within a short enough time interval, so that they cannot be resolved experimentally. The statistics of these periods may be altered by the dipole-dipole interaction between the atoms. There are a number of experiments with seemingly contradictory results on this question. For two and three $\mathrm{Ba}^{+}$ions a large number of double and triple jumps have been observed, two orders of magnitude more than expected for independent atoms [19, 20, 21]. Experiments with other ions showed no such effects [22]. In particular, none were seen for two $\mathrm{Hg}^{+}$ions [23]. More recently, effects similar to that of reference [19] were found in an experiment with $\mathrm{Ca}^{+}$ions in a linear Paul trap [24], in contrast to other experiments with the same ion species $[25,26]$. Neither were cooperative effects found in an extensive analysis of two trapped $\mathrm{Sr}^{+}$ions [27].

Theoretically, the qualitative explanation of such large cooperative effects for distances of the order of ten wavelengths of the strong transition proved to be difficult $[28,29,30,31]$. In particular, Lewenstein and Javanainen showed that for incoherent population of the meta-stable state and in the limit of infinitely strong driving cooperative effects occur only for inter-atomic distances smaller than a wavelength of the strong transition $[32,33]$. Skornia et al. recently put forward a new proposal for observing the dipole-dipole interaction of two V systems, based on the individual detection of the light emitted from each atom [34].

For two V systems numerical [35] and analytical [17] investigations of the effect of the dipole-dipole interaction showed an increase of up to $30 \%$ in the double jump rate when compared to independent systems. However, the level systems used in the experimental setups of References $[3,19,23]$ were not $\mathrm{V}$ systems so that a direct comparison between theory and experiment was not possible.

In this part of the present work, the main goal is to develop a theoretical description of the relevant experimental level systems. Explicit experimental data for the high double jump rate of Reference [19] was given for three $\mathrm{Ba}^{+}$ions only. In order to decide whether this can be explained by the dipole-dipole interaction, it is particularly important to achieve a realistic description for this case. The main result of this part is the analytical calculation of the transition rates between the different intensity periods for two and three atoms and different experimentally relevant level schemes. The cooperative effects are shown to be noticeable for suitable parameters but negligible in the case of the experiments.

The structure of this part is as follows: In Chapter 2, the theoretical description of macroscopic quantum jumps is explained. In Section 2.1 the quantum jump 
approach is introduced. Furthermore, the connection to the optical Bloch equations is established and explicit expressions for a general three-level system are given. In Section 2.2 the theoretical description of macroscopic bright and dark periods for a single atom is reviewed. Chapter 3 contains the treatment of two and three dipoledipole interacting atoms with different relevant level configurations. Following the derivation in Reference [36] the generalization of the quantum jump approach to dipole-interacting multi-level systems is presented in Section 3.1. In Section 3.2 the results for two atoms are presented. First, the derivation of the transition rates for two V systems as given in Reference [17] is explained. This approach is extended first to the case of two D systems for the description of the $\mathrm{Hg}^{+}$ions and then to the case of two four level systems for $\mathrm{Ba}^{+}$ions. In each case a discussion of the results is given including a comparison with the results of reference [37]. In Section 3.3 the results for three dipole-interacting atoms are presented. First, the conditional Hamiltonian and the reset state for this case are derived. Due to the increasing complexity group theoretical methods have to be applied exploiting symmetries inherent in the system in order to reduce the dimensionality of the equations involved. For the three $\mathrm{V}$ systems and three $\mathrm{D}$ systems the transition rates and the double and triple jump rates are calculated and discussed. For increasing level numbers the method becomes increasingly laborious, but fortunately insights stemming from a structural analysis allowed a surprising simplification which led to explicit results for this case. 


\section{Chapter 2}

\section{Quantum jumps in the fluorescence of single atoms}

\subsection{The quantum jump approach}

The quantum jump approach by Hegerfeldt and Wilser $[12,13,11,14]$ has been developed to describe quantum optical experiments with single or few quantum systems. It is equivalent to the Monte Carlo-wave function method of Dalibard, Castin and Mølmer [16] and the method of quantum trajectories by Carmichael [15]. A detailed review about this method and major applications can be found in Reference [38].

\subsubsection{The conditional Hamiltonian}

In the following a laser-driven single atom subject to the free quantized radiation field will be considered. Let $H$ be the Hamiltonian of the whole system, atom and radiation field. It consists of the free part $H_{0}=H_{\mathrm{A}}+H_{\mathrm{F}}$ for atom and field and an interaction term $H_{1}$ including the coupling to the laser, which is treated classically, and the free field. In the atomic Hamiltonian $H_{\mathrm{A}}$ the translational degrees of freedom are disregarded since the atoms are assumed to be located at a fixed positions in the trap in this part of the work. The state of the system is given by the product of the atomic state $|\psi\rangle$ and the state of the radiation field in Fock basis $\left|n_{\boldsymbol{k}, \lambda}\right\rangle$, with wave vector $\boldsymbol{k}$ and polarization $\lambda$. The time development is described by the time development operator $U\left(t^{\prime}, t\right)$.

For this system the emission of photons shall be observed. In order to avoid the problems of continuous measurements, discrete gedanken measurements in time intervals $\Delta t$ are being performed. For this purpose an ideal $4 \pi$ detector is assumed which collects every emitted photon. The time interval $\Delta t$ has to fulfill certain bounds. On the one hand it has to be short compared to the lifetimes of the atomic states involved. These are of the order of $10^{-8} \mathrm{~s}$. On the other hand $\Delta t$ has to be 
large enough to avoid a freezing of the atom's time evolution due to the quantum Zeno effect $[36,39]$. For optical transitions this leads to

$$
\Delta t \approx 10^{-12} \mathrm{~s} .
$$

At time $t=0$ the system is in the state $\left|0_{\mathrm{ph}}\right\rangle\left|\psi_{0}\right\rangle$. After a time $\Delta t$ it has evolved to the state $U(\Delta t, 0)\left|0_{\mathrm{ph}}\right\rangle\left|\psi_{0}\right\rangle$. Now the first gedanken measurement is performed. If no photon has been detected this corresponds to a projection onto the zero photon subspace with the projector $\mathbb{P}_{0}=\left|0_{\mathrm{ph}}\right\rangle\left\langle 0_{\mathrm{ph}}\right|[40]$. After the measurement the system is therefore in the state

$$
\mathbb{P}_{0} U(\Delta t, 0)\left|0_{\mathrm{ph}}\right\rangle\left|\psi_{0}\right\rangle=\left|0_{\mathrm{ph}}\right\rangle\left\langle 0_{\mathrm{ph}}|U(\Delta t, 0)| 0_{\mathrm{ph}}\right\rangle\left|\psi_{0}\right\rangle
$$

in this case.

Accordingly, the state of the system at time $t_{n}=n \Delta t$ is given by

$$
\begin{aligned}
\left|0_{\mathrm{ph}}\right\rangle\left|\psi_{\text {cond }}\left(t_{n}\right)\right\rangle & =\mathbb{P}_{0} U\left(t_{n}, t_{n-1}\right) \mathbb{P}_{0} \ldots \mathbb{P}_{0} U\left(t_{2}, t_{1}\right) \mathbb{P}_{0} U\left(t_{1}, t_{0}\right)\left|0_{\mathrm{ph}}\right\rangle\left|\psi_{0}\right\rangle \\
& =\left|0_{\mathrm{ph}}\right\rangle U_{\text {cond }}^{\mathrm{S}}\left(t_{n}, t_{0}\right)\left|\psi_{0}\right\rangle
\end{aligned}
$$

if no photon has been detected up to this time. Here the conditional time-development operator in the Schrödinger picture

$$
U_{\text {cond }}^{\mathrm{S}}\left(t_{n}, t_{0}\right)=\left\langle 0_{\mathrm{ph}}\left|U\left(t_{n}, t_{n-1}\right)\right| 0_{\mathrm{ph}}\right\rangle\left\langle 0_{\mathrm{ph}}|\ldots| 0_{\mathrm{ph}}\right\rangle\left\langle 0_{\mathrm{ph}}\left|U\left(t_{1}, t_{0}\right)\right| 0_{\mathrm{ph}}\right\rangle
$$

has been defined. It describes the time development of the atomic state under the condition that no photon has been detected. In the interaction picture with respect to $H_{1}$ it can be written as

$$
\begin{aligned}
U_{\text {cond }}\left(t_{i}, t_{i-1}\right) & =\left\langle 0_{\mathrm{ph}}\left|U_{\mathrm{I}}\left(t_{i}, t_{i-1}\right)\right| 0_{\mathrm{ph}}\right\rangle \\
& =\mathrm{e}^{\mathrm{i} H_{\mathrm{A}} t_{i} / \hbar}\left\langle 0_{\mathrm{ph}}\left|U\left(t_{i}, t_{i-1}\right)\right| 0_{\mathrm{ph}}\right\rangle \mathrm{e}^{-\mathrm{i} H_{\mathrm{A}} t_{i-1} / \hbar} .
\end{aligned}
$$

Using the Hamiltonian in the interaction picture $H_{\mathrm{I}}(t)=\mathrm{e}^{\mathrm{i} H_{0} t / \hbar} H_{1} \mathrm{e}^{-\mathrm{i} H_{0} t / \hbar}$ the conditional time-development operator $U_{\text {cond }}\left(t_{i}, t_{i-1}\right)$ can be calculated by expanding $U_{\mathrm{I}}$ into its Dyson series yielding

$$
\begin{aligned}
U_{\text {cond }}\left(t_{i}, t_{i-1}\right)= & \mathbb{1}_{\mathrm{A}}-\frac{\mathrm{i}}{\hbar} \int_{t_{i-1}}^{t_{i}} \mathrm{~d} t^{\prime}\left\langle 0_{\mathrm{ph}}\left|H_{\mathrm{I}}\left(t^{\prime}\right)\right| 0_{\mathrm{ph}}\right\rangle \\
& -\frac{1}{\hbar^{2}} \int_{t_{i-1}}^{t_{i}} \mathrm{~d} t^{\prime} \int_{t_{i-1}}^{t^{\prime}} \mathrm{d} t^{\prime \prime}\left\langle 0_{\mathrm{ph}}\left|H_{\mathrm{I}}\left(t^{\prime}\right) H_{\mathrm{I}}\left(t^{\prime \prime}\right)\right| 0_{\mathrm{ph}}\right\rangle+\mathcal{O}\left(\Delta t^{2}\right),
\end{aligned}
$$

where $\mathbb{1}_{\mathrm{A}}$ is the unity operator on the atomic subspace. The conditional time development is generated by a conditional Hamiltonian $H_{\text {cond }}$, so that

$$
U_{\text {cond }}\left(t, t_{0}\right)=\mathrm{e}^{-\mathrm{i} H_{\text {cond }}\left(t-t_{0}\right) / \hbar} .
$$


The conditional Hamiltonian is non-Hermitian since the norm squared of the conditionally time-developed state is diminishing. It gives the probability of detecting no photon up to time $t$

$$
P_{0}\left(t ; \psi_{0}\right)=\|\left|\psi_{\text {cond }}(t)\right\rangle\left\|^{2}=\right\| \mathrm{e}^{-\mathrm{i} H_{\text {cond }} t / \hbar}\left|\psi_{0}\right\rangle \|^{2} .
$$

The extension of this formalism to density matrices is obvious. If $\rho_{0}$ denotes the state of the atom at time $t=0$ then

$$
\rho_{\text {cond }}(t)=U_{\text {cond }}(t, 0) \rho_{0} U_{\text {cond }}^{\dagger}(t, 0)
$$

gives the conditionally developed density matrix at time $t$ and

$$
P_{0}\left(t ; \rho_{0}\right)=\operatorname{Tr} \rho_{\text {cond }}(t) .
$$

Besides the zero-photon probability $P_{0}\left(t ; \psi_{0}\right)$ another quantity which is often needed is the probability distribution of the first photon $w_{1}\left(t ; \psi_{0}\right)$. It is given by the negative derivative of the no-photon probability

$$
w_{1}\left(t ; \psi_{0}\right)=-\frac{\mathrm{d}}{\mathrm{d} t} P_{0}\left(t ; \psi_{0}\right) .
$$

\subsubsection{The reset state}

In the previous section the time development of the atom under the condition that no photon has been detected was addressed. Now the state of the atom after the detection of a photon will be derived. This question is discussed in detail in Reference [13].

Let the state of the whole system at time $t$ be in the state $\rho_{\mathrm{AF}}(t)=\left|0_{\mathrm{ph}}\right\rangle \rho(t)\left\langle 0_{\mathrm{ph}}\right|$, where $\rho(t)$ is the density matrix of the atomic state. Then at time $t+\Delta t$ it will be in the state

$$
\rho_{\mathrm{AF}}(t+\Delta t)=U_{\mathrm{I}}(t+\Delta t, t)\left|0_{\mathrm{ph}}\right\rangle \rho(t)\left\langle 0_{\mathrm{ph}}\right| U_{\mathrm{I}}^{\dagger}(t+\Delta t, t) .
$$

Now, again, a gedanken measurement is carried out. Since $\Delta t$ is short compared to the lifetimes of the energy levels involved, it is very unlikely to find more than one photon in a measurement. So, if a photon was detected, one has to project onto the one-photon subspace and the state after the measurement is

$$
\mathbb{P}_{1} U_{\mathrm{I}}(t+\Delta t, t)\left|0_{\mathrm{ph}}\right\rangle \rho(t)\left\langle 0_{\mathrm{ph}}\right| U_{\mathrm{I}}^{\dagger}(t+\Delta t, t) \mathbb{P}_{1},
$$

with $\mathbb{P}_{1}:=\sum_{\boldsymbol{k}, \lambda}\left|1_{\boldsymbol{k}, \lambda}\right\rangle\left\langle 1_{\boldsymbol{k}, \lambda}\right|$. Here it is assumed that a photon does not interact with the atom anymore when it is absorbed in the detector. The state of the atom after a photon detection arises by taking the partial trace with respect to the radiation field

$$
\operatorname{Tr}_{\mathrm{ph}}\left(\mathbb{P}_{1} \rho_{\mathrm{AF}}(t+\Delta t) \mathbb{P}_{1}\right)=\sum_{\boldsymbol{k}, \lambda}\left\langle 1_{\boldsymbol{k}, \lambda}\left|U_{\mathrm{I}}(t+\Delta t, t)\right| 0_{\mathrm{ph}}\right\rangle \rho(t)\left\langle 0_{\mathrm{ph}}\left|U_{\mathrm{I}}^{\dagger}(t+\Delta t, t)\right| 1_{\boldsymbol{k}, \lambda}\right\rangle .
$$


Expanding the time development operators into a Dyson series and keeping only first order terms in $\Delta t$ gives the unnormalized reset state $\mathscr{R}(\rho)$

$$
\begin{aligned}
& \operatorname{Tr}_{\mathrm{ph}}\left(\mathbb{P}_{1} \rho(t+\Delta t) \mathbb{P}_{1}\right) \\
&=\frac{1}{\hbar^{2}} \int_{t}^{t+\Delta t} \mathrm{~d} t^{\prime} \int_{t}^{t+\Delta t} \mathrm{~d} t^{\prime \prime} \sum_{\boldsymbol{k}, \lambda}\left\langle 1_{\boldsymbol{k}, \lambda}\left|H_{\mathrm{I}}\left(t^{\prime}\right)\right| 0_{\mathrm{ph}}\right\rangle \rho(t)\left\langle 0_{\mathrm{ph}}\left|H_{\mathrm{I}}\left(t^{\prime \prime}\right)\right| 1_{\boldsymbol{k}, \lambda}\right\rangle+\mathcal{O}\left(\Delta t^{2}\right) \\
&=\mathscr{R}(\rho) \Delta t+\mathcal{O}\left(\Delta t^{2}\right) .
\end{aligned}
$$

The normalized reset state is given by

$$
\widehat{\mathscr{R}}(\rho):=\frac{\mathscr{R}(\rho)}{\operatorname{Tr}(\mathscr{R}(\rho))},
$$

where $\operatorname{Tr}(\mathscr{R}(\rho))$ is the probability of detecting a photon after the time interval $\Delta t$. It can be used again as an initial state for a subsequent time development with $U_{\text {cond }}$. By generating random photon emissions in accordance with the first photon distribution $w_{1}\left(t ; \psi_{0}\right)$ the quantum jump approach thus allows to simulate fluorescence trajectories of single atoms. An efficient algorithm for this purpose is given, for example in Reference [36].

\subsubsection{The optical Bloch equation}

A given ensemble of atoms which at time $t$ is described by $\rho(t)$ can be divided at time $t+\Delta t$ into two sub-ensembles. One contains all atoms which have emitted a photon and the other contains the atoms which have not emitted a photon. The former is described by the reset state, and the latter is given by $\rho_{\text {cond }}(t+\Delta t)$. For the whole ensemble one therefore has

$$
\begin{aligned}
\rho(t+\Delta t) & =U_{\text {cond }}(t+\Delta t, t) \rho(t) U_{\text {cond }}^{\dagger}(t+\Delta t, t)+\mathscr{R}(\rho(t)) \Delta t \\
& =\left(\mathbb{1}_{\mathrm{A}}-\frac{\mathrm{i}}{\hbar} H_{\text {cond }} \Delta t\right) \rho(t)\left(\mathbb{1}_{\mathrm{A}}+\frac{\mathrm{i}}{\hbar} H_{\text {cond }}^{\dagger} \Delta t\right)+\mathscr{R}(\rho(t)) \Delta t \\
& =\rho(t)-\frac{\mathrm{i}}{\hbar}\left[H_{\text {cond }} \rho(t)-\rho(t) H_{\text {cond }}^{\dagger}\right] \Delta t+\mathscr{R}(\rho(t)) \Delta t
\end{aligned}
$$

up to first order in $\Delta t$. Since $\Delta t$ is much smaller than the time scale on which $\rho(t)$ evolves one has

$$
\dot{\rho}(t)=-\frac{\mathrm{i}}{\hbar}\left[H_{\text {cond }} \rho(t)-\rho(t) H_{\text {cond }}^{\dagger}\right]+\mathscr{R}(\rho(t))
$$

This is the master equation for the atomic state of an atom interacting with the radiation field also known as the optical Bloch equation. It is usually derived in a different way without the use of gedanken measurements [41]. It can be shown that both approaches yield the same result [13]. 


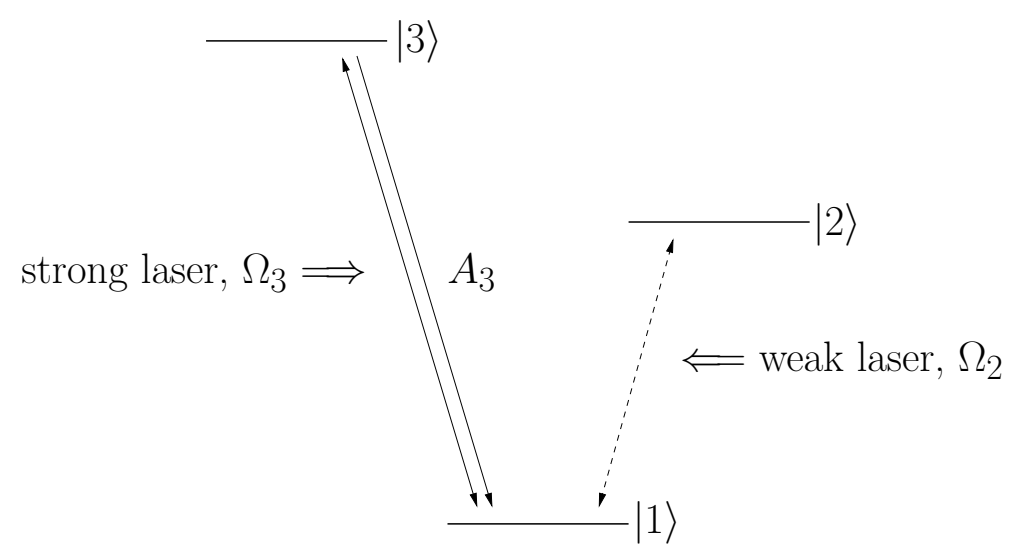

Figure 2.1: Three-level system in V configuration.

The right hand side of equation (2.18) can be used as a definition for the Liouville super-operator $\mathscr{L}$

$$
\dot{\rho}=\mathscr{L} \rho,
$$

which immediately gives the formal solution

$$
\rho(t)=\mathrm{e}^{\mathscr{L} t} \rho\left(t_{0}\right) .
$$

\subsubsection{Application to three-level systems}

In the following sections the conditional Hamiltonian and the reset state for threelevel systems in different configurations are needed. The corresponding calculations will be given here, as an example, for the $\mathrm{V}$-system as shown in Figure 2.1, in order to illustrate the necessary steps.

The free Hamiltonian for atom and radiation field is given by

$$
H_{0}=\hbar \omega_{3}|3\rangle\left\langle 3\left|+\hbar \omega_{2}\right| 2\right\rangle\langle 2|+\sum_{\boldsymbol{k} \lambda} \hbar \omega_{k} a_{\boldsymbol{k} \lambda}^{\dagger} a_{\boldsymbol{k} \lambda}
$$

where $\omega_{2}$ and $\omega_{3}$ are the transition frequencies between the ground state and the excited states, $a_{\boldsymbol{k} \lambda}^{\dagger}$ and $a_{\boldsymbol{k} \lambda}$ are the raising and lowering operators for the field mode with wave vector $\boldsymbol{k}$, polarization $\lambda$, and angular frequency $\omega_{k}=c|\boldsymbol{k}|$. The interaction Hamiltonian in dipole approximation reads

$$
H_{1}=\boldsymbol{D} \cdot\left(\boldsymbol{E}(\boldsymbol{r})+\boldsymbol{E}_{\mathrm{L}}(\boldsymbol{r}, t)\right) .
$$

with the dipole operator

$$
\boldsymbol{D}=\boldsymbol{D}_{12}|1\rangle\left\langle 2\left|+\boldsymbol{D}_{13}\right| 1\right\rangle\langle 3|+\text { h.c. }
$$

using the dipole transition matrix elements $\boldsymbol{D}_{i j}=e\langle i|\hat{\boldsymbol{x}}| j\rangle$. The field terms are given by

$$
\boldsymbol{E}(\boldsymbol{r})=\mathrm{i} \sum_{\boldsymbol{k} \lambda} \sqrt{\frac{\hbar \omega_{k}}{2 \epsilon_{0} V}}\left(\boldsymbol{\epsilon}_{\boldsymbol{k} \lambda} a_{\boldsymbol{k} \lambda} \mathrm{e}^{\mathrm{i} \boldsymbol{k} \cdot \boldsymbol{r}}+\boldsymbol{\epsilon}_{\boldsymbol{k} \lambda}^{*} a_{\boldsymbol{k} \lambda}^{\dagger} \mathrm{e}^{-\mathrm{i} \boldsymbol{k} \cdot \boldsymbol{r}}\right)
$$


for the free radiation field and

$$
\boldsymbol{E}_{\mathrm{L}}(\boldsymbol{r}, t)=\operatorname{Re}\left(\boldsymbol{E}_{0, \mathrm{~L}_{2}} \mathrm{e}^{\mathrm{i}\left(\boldsymbol{k}_{\mathrm{L}_{2}} \cdot \boldsymbol{r}-\omega_{\mathrm{L}_{2}} t\right)}\right)+\operatorname{Re}\left(\boldsymbol{E}_{0, \mathrm{~L}_{3}} \mathrm{e}^{\mathrm{i}\left(\boldsymbol{k}_{\mathrm{L}_{3}} \cdot \boldsymbol{r}-\omega_{\mathrm{L}_{3}} t\right)}\right) .
$$

for the laser fields. Defining the Rabi frequencies as

$$
\Omega_{j}=\frac{e}{\hbar}\left(\boldsymbol{E}_{0, \mathrm{~L}_{j}} \cdot \boldsymbol{D}_{1 j}\right)
$$

and the coupling constants for the interaction between the radiation field and the atom as

$$
g_{\boldsymbol{k \lambda}}^{(j)}=\mathrm{i} e \sqrt{\frac{\omega_{j}}{2 \epsilon_{0} \hbar V}}\left(\boldsymbol{D}_{1 j} \cdot \boldsymbol{\epsilon}_{\boldsymbol{k} \lambda}\right)
$$

one has for the Hamiltonian

$$
\begin{aligned}
H= & H_{0}+H_{1} \\
= & \sum_{j=2}^{3} \hbar \omega_{j}|j\rangle\langle j|+\sum_{\boldsymbol{k} \lambda} \hbar \omega_{k} a_{\boldsymbol{k} \lambda}^{\dagger} a_{\boldsymbol{k} \lambda}+\sum_{j=2}^{3} \sum_{\boldsymbol{k} \lambda}\left[\hbar g_{\boldsymbol{k} \lambda}^{(j)} a_{\boldsymbol{k} \lambda}|j\rangle\langle 1|+\text { h.c. }\right] \\
& +\sum_{j=2}^{3} \frac{\hbar \Omega_{j}}{2}\left[|1\rangle\langle j| \mathrm{e}^{\mathrm{i} \omega_{\mathrm{L}_{j}} t}+\text { h.c. }\right] .
\end{aligned}
$$

In the laser adapted interaction picture with respect to $H_{0}^{\mathrm{L}}=\hbar \omega_{\mathrm{L}_{2}}|2\rangle\left\langle 2\left|+\hbar \omega_{\mathrm{L}_{3}}\right| 3\right\rangle\langle 3|+$ $\sum_{\boldsymbol{k} \lambda} \hbar \omega_{k} a_{\boldsymbol{k} \lambda}^{\dagger} a_{\boldsymbol{k} \lambda}$ the Hamiltonian can be written as

$$
\begin{aligned}
H_{\mathrm{I}}= & \mathrm{e}^{\mathrm{i} H_{0}^{\mathrm{L}} t / \hbar} H_{1}^{\mathrm{L}} \mathrm{e}^{-\mathrm{i} H_{0}^{\mathrm{L}} t / \hbar} \\
= & \sum_{j=1}^{2} \sum_{\boldsymbol{k} \lambda} \hbar\left[g_{\boldsymbol{k} \lambda}^{(j)} a_{\boldsymbol{k} \lambda} \mathrm{e}^{-\mathrm{i}\left(\omega_{k}-\omega_{j}\right) t}|j\rangle\langle 1|+\text { h.c. }\right] \\
& +\sum_{j=2}^{3} \frac{\hbar}{2} \Omega_{j}[|1\rangle\langle j|+| j\rangle\langle 1|]-\sum_{j=2}^{3} \hbar \Delta_{j}|j\rangle\langle j|,
\end{aligned}
$$

where the laser detunings $\Delta_{j}=\omega_{\mathrm{L}_{j}}-\omega_{j}$ have been introduced. For the calculation of the conditional Hamiltonian the time development operator $U_{\text {cond }}\left(t_{i}, t_{i-1}\right)$ has to be determined to first order in $\Delta t$, according to equation (2.6). In first order in $H_{\mathrm{I}}$, the atom-field interaction term drops out due to the field operators. One has

$$
-\frac{\mathrm{i}}{\hbar} \int_{t_{i-1}}^{t_{i}} \mathrm{~d} t^{\prime}\left\langle 0_{\mathrm{ph}}\left|H_{\mathrm{I}}\left(t^{\prime}\right)\right| 0_{\mathrm{ph}}\right\rangle=-\frac{\mathrm{i}}{\hbar} \sum_{j=2}^{3}\left[\frac{\hbar \Omega_{j}}{2}[|1\rangle\langle j|+| j\rangle\langle 1|]-\hbar \Delta_{j}|j\rangle\langle j|\right] \Delta t .
$$

The corresponding term in second order is discarded since it is proportional to $\Delta t^{2}$. After some calculation, the residual terms lead to (see, e.g., Reference [12])

$$
-\frac{1}{\hbar^{2}} \int_{t_{i-1}}^{t_{i}} \mathrm{~d} t^{\prime} \int_{t_{i-1}}^{t^{\prime}} \mathrm{d} t^{\prime \prime}\left\langle 0_{\mathrm{ph}}\left|H_{\mathrm{I}}\left(t^{\prime}\right) H_{\mathrm{I}}\left(t^{\prime \prime}\right)\right| 0_{\mathrm{ph}}\right\rangle=-\sum_{j=2}^{3} \frac{A_{j}}{2}|j\rangle\langle j| \Delta t+\mathcal{O}\left(\Delta t^{2}\right) .
$$


Here the Einstein coefficients

$$
A_{j}=\frac{e^{2} \omega_{j}^{3}\left|\boldsymbol{D}_{1 j}\right|^{2}}{3 \pi \epsilon_{0} \hbar c^{3}}
$$

have been defined. Overall, the conditional Hamiltonian for the V system reads

$$
H_{\text {cond }}=\sum_{j=2}^{3} \frac{\hbar \Omega_{j}}{2}[|1\rangle\langle j|+\text { h.c. }]-\sum_{j=2}^{3} \frac{\hbar}{2}\left(\mathrm{i} A_{j}+2 \Delta_{j}\right)|j\rangle\langle j| \text {. }
$$

\section{The reset state}

The reset state for a single $\mathrm{V}$-system can be determined without calculation. After a photon emission the system is always in the ground state. The normalized reset state is therefore

$$
\hat{\mathscr{R}}(\rho)=|1\rangle\langle 1| \text {. }
$$

The probability for an emission in a particular state is given by the population of the state multiplied with the corresponding Einstein coefficient, which implies the non-normalized reset state

$$
\mathscr{R}(\rho)=A_{2}|1\rangle\langle 2|\rho| 2\rangle\left\langle 1\left|+A_{3}\right| 1\right\rangle\langle 3|\rho| 3\rangle\langle 1|
$$

\subsection{Description of the fluorescence properties}

The quantum jump approach allows the description of the fluorescence process of single quantum systems. In this work particular interest is put in the effect of macroscopic bright and dark periods which can only be observed in single quantum systems. In this section the mechanism which leads to this effect is explained in more detail.

The occurrence of macroscopic bright and dark periods in the fluorescence of a single quantum system can be explained by means of the quantum jump approach on the grounds of quantum mechanics. This approach also allows to describe the statistics of these periods. In the following a brief explanation of the basic principles of these calculations will be given. A more detailed discussion can be found in Reference [12].

A three-level system shows macroscopic quantum jumps if one of the excited states is meta-stable and driven by a weak laser ${ }^{1}$. For the $\mathrm{V}$ system this means

$$
\Omega_{2}, A_{2} \ll \Omega_{3}, A_{3}
$$

\footnotetext{
${ }^{1}$ For macroscopic quantum jumps without a meta-stable state see Reference [42].
} 
In the case of the $\mathrm{V}$ system the atom is in the ground state after each photon emission. Hence, the probability that no photon is detected until time $t$ after this emission is given by the zero-photon probability

$$
P_{0}(t ;|1\rangle)=\| \mathrm{e}^{-\mathrm{i} H_{\text {cond }} t / \hbar}|1\rangle \|^{2} .
$$

With $M \equiv \mathrm{i} H_{\text {cond }} / \hbar$ this can be written as

$$
P_{0}(t ;|1\rangle)=\| \sum_{j=1}^{3} \sum_{k=1}^{3}|j\rangle a_{j k} \mathrm{e}^{-\mu_{k} t} \|^{2}=\sum_{j=1}^{3}\left|\sum_{k=1}^{3} a_{j k} \mathrm{e}^{-\mu_{k} t}\right|^{2},
$$

where

$$
a_{j k}:=\left\langle j\left|\prod_{l(\neq k)} \frac{M-\mu_{l}}{\mu_{k}-\mu_{l}}\right| 1\right\rangle .
$$

The $\mu_{j}$ are the eigenvalues of $M$. They are given, to good approximation, by

$$
\mu_{1,2}=\frac{1}{4}\left(A_{3}-2 \mathrm{i} \Delta_{3} \pm \sqrt{\left(A_{3}-2 \mathrm{i} \Delta_{3}\right)^{2}-4 \Omega_{3}^{2}}\right), \quad \mu_{3}=\frac{A_{2}}{2}+\Omega_{2}^{2} \frac{A_{3}-2 \mathrm{i} \Delta_{3}}{2 \Omega_{3}^{2}} .
$$

For simplicity, resonant excitation of the weak transition $\left(\Delta_{2}=0\right)$ has been assumed here. Since $\operatorname{Re} \mu_{3} \ll \operatorname{Re} \mu_{1,2}$ the probability $P_{0}(t,|1\rangle)$ can be divided into a fastly decreasing part $P_{0}^{(1)}(t)$ and a slowly decreasing part $P_{0}^{(2)}(t)$. This bi-exponential form is the reason for the occurrence of macroscopic dark periods. Due to $P_{0}^{(1)}(t)$ it is very likely that the time between two successive photons is very small. But from time to time it may happen that no photon has been emitted up to a time where $P_{0}^{(1)}(t)$ has already vanished. Then only $P_{0}^{(2)}(t)$ plays a role leading to a long dark period. The state of the system is given by the corresponding eigenstate $\left|\mu_{3}\right\rangle$. It can be shown that this state is given to good approximation by $|2\rangle$, with small contributions of the other two states [39, 43].

Consequently, the dark periods can be defined by introducing a time constant $t_{\mathrm{D}}$ for which the condition

$$
\frac{1}{2 \operatorname{Re} \mu_{1,2}} \ll t_{\mathrm{D}} \ll \frac{1}{2 \operatorname{Re} \mu_{3}}
$$

is posed. A dark period is then defined as period without photon emission which lasts longer than $t_{\mathrm{D}}$. Accordingly, the probability that after a given photon emission there is a dark period is given by $p=P_{0}\left(t_{\mathrm{D}}\right)$. As long as $(2.41)$ holds this probability is independent of the actual (specific) choice of $t_{\mathrm{D}}$. For the $\mathrm{V}$ system it is given by

$$
p=\Omega_{2}^{2} \frac{A_{3}^{2}+4 \Delta_{3}^{2}}{\Omega_{3}^{4}}+A_{2} .
$$

Splitting the first photon distribution $w_{1}(t)$ into a part for $t<t_{\mathrm{D}}$ and a part for $t \geq t_{\mathrm{D}}$ one can define a probability density

$$
w_{1 \mathrm{~L}}(t)=\frac{1}{1-p} \chi_{\left[0, t_{\mathrm{D}}\right)}(t) w_{1}(t)
$$


describing the distribution of the time between two emissions during a bright period and a density

$$
w_{\mathrm{D}}(t)=\frac{1}{p} \chi_{\left[t_{\mathrm{D}}, \infty\right)}(t) w_{1}(t)
$$

describing the distribution of the dark periods. The latter is approximately given by

$$
\begin{aligned}
w_{\mathrm{D}}(t) & =-\chi_{\left[t_{\mathrm{D}}, \infty\right)}(t) \frac{1}{p} \frac{\mathrm{d}}{\mathrm{d} t} P_{0}^{(2)}(t)=-\chi_{\left[t_{\mathrm{D}}, \infty\right)}(t) \frac{1}{p} \frac{\mathrm{d}}{\mathrm{d} t} p \mathrm{e}^{-2 \operatorname{Re} \mu_{3} t} \\
& =\chi_{\left[t_{\mathrm{D}}, \infty\right)}(t) 2 \operatorname{Re} \mu_{3} \mathrm{e}^{-2 \operatorname{Re} \mu_{3} t} .
\end{aligned}
$$

Neglecting $A_{2}$, the mean duration of a dark period is thus given by

$$
T_{\mathrm{D}}=\int_{0}^{\infty} \mathrm{d} t w_{\mathrm{D}}(t) t=\frac{1}{2 \operatorname{Re} \mu_{3}}=\frac{\Omega_{3}^{2}}{A_{3} \Omega_{2}^{2}}
$$

and its inverse can be interpreted as the transition rate from dark to bright period

$$
p_{01}=\frac{A_{3} \Omega_{2}^{2}}{\Omega_{3}^{2}} .
$$

A similar, though slightly more complicated, calculation using $w_{1 \mathrm{~L}}$ gives the transition rate from bright to dark period $p_{10}[12]$. More heuristically, this transition rate should be given by multiplying $p$ with the mean photon density in a bright period. Since during a bright period mainly the states $|1\rangle$ and $|3\rangle$ are populated and the system basically behaves like a two-level system one can use to good approximation the steady state photon rate of the two level system [12]

$$
I_{\mathrm{ss}}^{2 \mathrm{~L}}=\frac{A_{3} \Omega_{3}^{2}}{A_{3}^{2}+2 \Omega_{3}^{2}+4 \Delta_{3}^{2}}
$$

The transition rate from bright to dark period is given by

$$
p_{10}=p I_{\mathrm{ss}}^{2 \mathrm{~L}}=\frac{\Omega_{2}^{2} A_{3}^{3}}{\Omega_{3}^{2}\left(A_{3}^{2}+2 \Omega_{3}^{2}+4 \Delta_{3}^{2}\right)}=T_{\mathrm{L}}^{-1},
$$

where $T_{\mathrm{L}}$ is the mean duration of a bright period. The statistics of the bright and dark periods is described completely by the transition rates $p_{01}$ and $p_{10}$.

\subsection{Experimentally relevant level structures}

The level structures of the ions used in most of the relevant experiments cannot be described by the $\mathrm{V}$ system. In this section alternative level systems are presented which are better suited for the description of the experiments. 


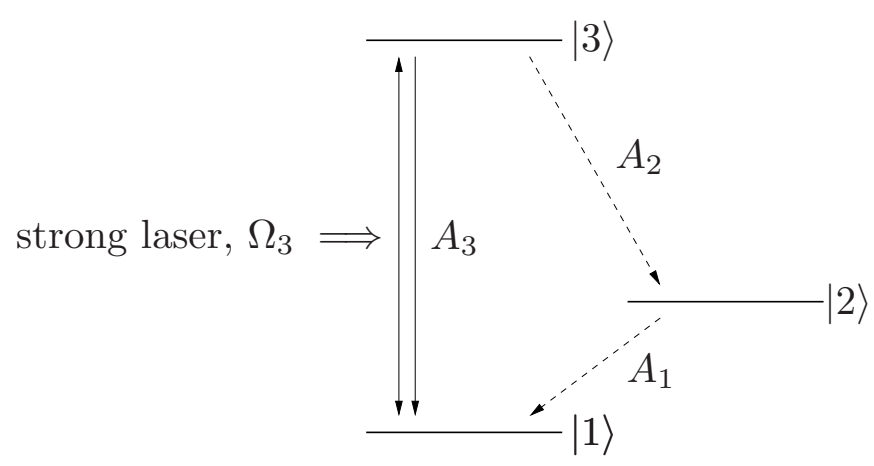

Figure 2.2: Three-level system in $\mathrm{D}$ configuration for the description of $\mathrm{Hg}^{+}$, with fast transitions (solid lines) and slow transitions (dashed lines).

\subsubsection{The D system}

The $\mathrm{Hg}^{+}$ions used in the experiments of References $[4,23]$ have a level structure in which the meta-stable state is populated via spontaneous decay and is not coupled by a laser. It can be modeled by the three-level system in a $\mathrm{D}$ configuration depicted in Figure 2.2. The transition $|1\rangle-|3\rangle$ is driven by a strong laser with Rabi frequency $\Omega_{3}$. The state $|3\rangle$ can decay via a fast transition, with Einstein coefficient $A_{3}$, back to the ground state but also via a slow decay with $A_{2}$ to the meta-stable level $|2\rangle$. For simplicity all transitions are treated as dipole transitions. This level itself can decay slowly back to the ground state. The condition

$$
A_{3}, \Omega_{3} \gg A_{1}, A_{2}
$$

enables the occurrence of macroscopic dark periods.

The level scheme of $\mathrm{Ca}^{+}$used in the experiments of references $[24,25,26]$ is a bit more complicated but the D system can also be used in this case.

\subsubsection{The four-level system for the description of the $\mathrm{Ba}^{+}$ ions}

The effective level scheme of the $\mathrm{Ba}^{+}$ions used in the experiment of References $[19,20]$ is more complicated. It is given by the 5-level system shown in Figure 2.3(a). The ground state $6{ }^{2} \mathrm{~S}_{1 / 2}$ and the two upper states $6{ }^{2} \mathrm{P}_{1 / 2}$ and $5^{2} \mathrm{D}_{5 / 2}$ constitute a strongly driven fluorescing $\Lambda$ system. The $\mathrm{P}_{1 / 2}$ level decays with a rate of about $3 \times 10^{7} \mathrm{~s}^{-1}$ to the $\mathrm{D}_{3 / 2}$ level and $10^{8} \mathrm{~s}^{-1}$ to the ground state. The photons from the transition of the ground state to the $\mathrm{P}_{1 / 2}$ state are recorded constituting the signal of the bright periods. An excitation by the weak incoherent driving of the $6{ }^{2} \mathrm{~S}_{1 / 2}$ - $6{ }^{2} \mathrm{P}_{3 / 2}$ transition and a subsequent decay to the meta-stable $5^{2} \mathrm{D}_{5 / 2}$ state leads to a dark period. This happens with a branching of about $1: 3$ since rate to the 

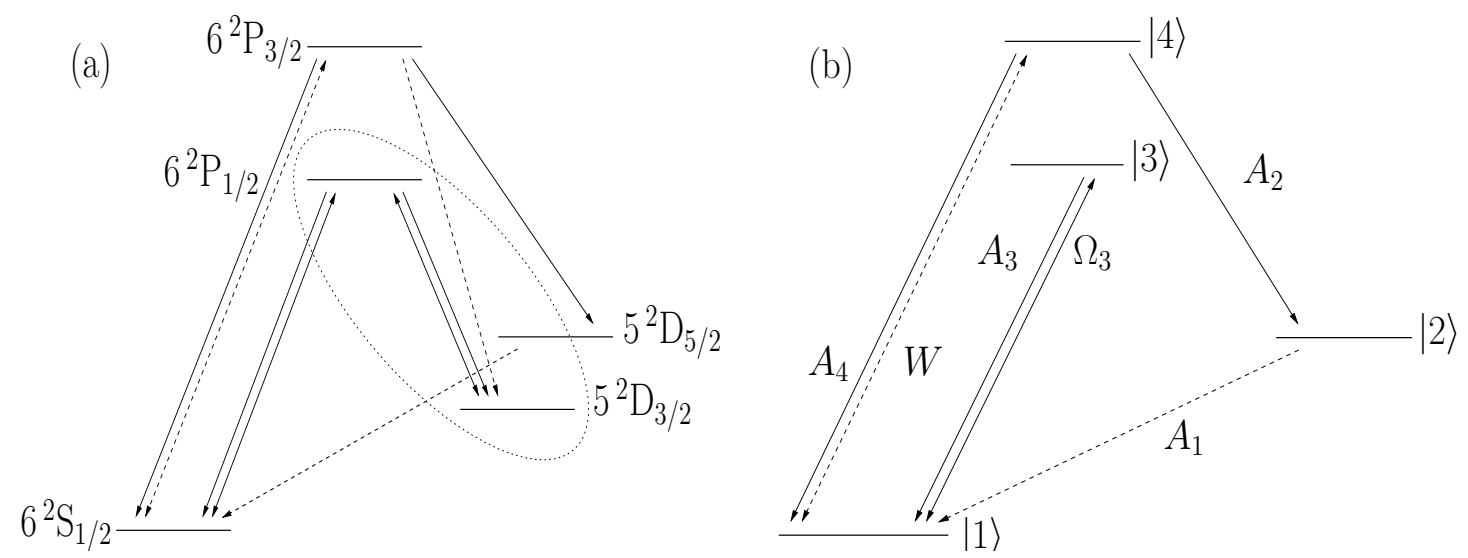

Figure 2.3: (a) Relevant level scheme of $\mathrm{Ba}^{+}$[19, 20]. For circled levels see text. (b) Effective four-level system for $B a^{+}$. Strong coherent driving of the $|1\rangle-|3\rangle$ transition by a laser, weak incoherent driving of the $|1\rangle-|4\rangle$ transition by a lamp, weak decay of level $|2\rangle$.

ground state is $1.2 \times 10^{8} \mathrm{~s}^{-1}$ and the rate to the meta-stable state is $4 \times 10^{7} \mathrm{~s}^{-1}$. The dark period ends when the ion decays back to the ground state which happens with a rate of $0.02 \mathrm{~s}^{-1}$. The laser driving the $\mathrm{S}_{1 / 2}-\mathrm{P}_{1 / 2}$ transition was detuned in the experiment by $\Delta=-1.5 \times 10^{8} \mathrm{~s}^{-1}$.

The transition to the dark state $D_{5 / 2}$ is only possible when the system is in the ground state. Therefore the details of the two upper states of the $\Lambda$ system, marked by the circle in Figure 2.3(a), play no significant role for the transition to a dark period, and therefore these two states are here replaced by an effective single level. This leads to the four-level configuration of Figure 2.3(b). 


\section{Chapter 3}

\section{Cooperative quantum jumps for two and three dipole-interacting atoms}

If more than one atom or ion is stored in a trap the dipole-dipole interaction between them may alter their radiative behavior. Such cooperative effects have attracted considerable interest in the literature (see $[17,18]$ and references therein), and they may play a role for possible quantum computers based on trapped ions or atoms. In the case of atoms showing macroscopic bright and dark periods in their fluorescence the dipole-dipole interaction may change the statistics of the jump process. In this context, different experimental results have been obtained which at first view seem to be contradictory. In an experiment with two and three $\mathrm{Ba}^{+}$ions the observation of a large number of double and triple jumps, exceeding by far the expected value for independent atoms, was reported [19]. These are jumps by two or three intensity steps within a short resolution time. Experiments with $\mathrm{Hg}^{+}$ions showed no such effects [23]. More recently, effects similar to Reference [19] were found in an experiment with $\mathrm{Ca}^{+}$ions [24], in contrast to another, comparable, experiment [25]. Neither were cooperative effects found experimentally in an extensive analysis of the quantum jump statistics of two trapped $\mathrm{Sr}^{+}$ions [27].

In this chapter the dipole-dipole interaction between two and three of the threeand four-level systems presented in the last chapter will be investigated. The aim is to describe the relevant experiments and to find out if cooperative effects as observed in the experiment of Reference [19] can be explained by the dipole-dipole interaction.

In Section 3.1 the extension of the quantum jump approach is presented. In Section 3.2 methods for the calculation of the transition rates between the different intensity periods for two $\mathrm{V}$ and $\mathrm{D}$ systems are presented and carried over to the fourlevel system for $\mathrm{Ba}^{+}$. In Section 3.3 these methods are used for the description of the three of the named three-level schemes. Finally a simplified approach is presented which also allows to gain analytical results for three four-level systems. 


\subsection{Quantum jump approach for dipole-interac- ting atoms}

The Hamiltonian for $n$ general three-level systems in dipole- and rotating-waveapproximation and in the interaction picture is given by

$$
\begin{aligned}
H_{\mathrm{I}}=\sum_{i=1}^{n} H_{\mathrm{I} i}= & \sum_{i=1}^{n} \sum_{j=2}^{3} \sum_{\boldsymbol{k} \lambda} \hbar\left[g_{j, \boldsymbol{k} \lambda}^{(i)} a_{\boldsymbol{k} \lambda} S_{i, j}^{+} \mathrm{e}^{\mathrm{i} \boldsymbol{k} \cdot \boldsymbol{r}_{i}} \mathrm{e}^{-\mathrm{i}\left(\omega_{k}-\omega_{j}\right) t}+\text { h.c. }\right] \\
& +\sum_{i=1}^{n} \sum_{j=2}^{3} \frac{\hbar}{2}\left[\Omega_{i j} S_{i j}^{-}+\text {h.c. }\right]-\sum_{i=1}^{n} \sum_{j=2}^{3} \hbar \Delta_{j} S_{i j}^{+} S_{i j}^{-},
\end{aligned}
$$

where $\boldsymbol{r}_{i}$ is the position of the $i$ th atom and

$$
S_{i 1}^{+}=|3\rangle_{i i}\left\langle 2\left|, \quad S_{i 2}^{+}=\right| 2\right\rangle_{i i}\left\langle 1\left|, \quad S_{i 3}^{+}=\right| 3\right\rangle_{i i}\langle 1|, \quad \text { and } \quad S_{i j}^{-}=S_{i j}^{+\dagger}
$$

are the raising and lowering operators for the different transitions of the $i$ th atom ${ }^{1}$. In the following the transition connected to the operators $S_{i j}^{ \pm}$will be referred to as transition $j$. Note that for two D systems the definitions of $S_{i 1}^{+}$and $S_{i 2}^{+}$have to be interchanged in order to retain the suggestive notation introduced for a single $\mathrm{D}$ system in the last chapter. The coupling constants between the atom and the free radiation field are given by

$$
g_{j, \boldsymbol{k} \lambda}^{(i)}:=\mathrm{i} e \sqrt{\frac{\omega_{k}}{2 \epsilon_{0} \hbar V}}\left(\boldsymbol{D}_{j}^{(i)} \cdot \boldsymbol{\epsilon}_{\boldsymbol{k} \lambda}\right)
$$

where $\boldsymbol{D}_{j}^{(i)}$ is the dipole transition matrix element for the transition $j$. The Rabi frequencies are defined as

$$
\Omega_{i j}:=\frac{e}{\hbar}\left(\boldsymbol{E}_{0, \mathrm{~L}_{j}} \cdot \boldsymbol{D}_{j}^{(i)}\right) \mathrm{e}^{-\mathrm{i} \boldsymbol{k}_{\mathrm{L}_{j}} \cdot \boldsymbol{r}_{i}}
$$

with $\boldsymbol{E}_{0, \mathrm{~L}_{j}}$ being the field amplitude of a laser field tuned to match the frequency of the transition $j$.

\footnotetext{
${ }^{1}$ For simplicity, the unity operators on the subspaces of the other atoms and of the radiation field are suppressed in the following.
} 


\subsubsection{The conditional Hamiltonian for dipole-interacting three-level systems}

The conditional Hamiltonian can be calculated as in section 2.1.1 using the Hamiltonian (3.1) and expanding the conditional time development operator

$$
\begin{aligned}
U_{\text {cond }}\left(t_{i}, t_{i-1}\right)= & \mathbb{1}_{\mathrm{A}}-\frac{\mathrm{i}}{\hbar} \int_{t_{i-1}}^{t_{i}} \mathrm{~d} t^{\prime}\left\langle 0_{\mathrm{ph}}\left|H_{\mathrm{I}}\left(t^{\prime}\right)\right| 0_{\mathrm{ph}}\right\rangle \\
& -\frac{1}{\hbar^{2}} \int_{t_{i-1}}^{t_{i}} \mathrm{~d} t^{\prime} \int_{t_{i-1}}^{t^{\prime}} \mathrm{d} t^{\prime \prime} \sum_{i, i^{\prime}=1}^{n}\left\langle 0_{\mathrm{ph}}\left|H_{\mathrm{I} i}\left(t^{\prime}\right) H_{\mathrm{I} i^{\prime}}\left(t^{\prime \prime}\right)\right| 0_{\mathrm{ph}}\right\rangle+\mathcal{O}\left(\Delta t^{2}\right) .
\end{aligned}
$$

The first order terms

$$
-\frac{\mathrm{i}}{\hbar} \int_{t_{i-1}}^{t_{i}} \mathrm{~d} t^{\prime}\left\langle 0_{\mathrm{ph}}\left|H_{\mathrm{I}}\left(t^{\prime}\right)\right| 0_{\mathrm{ph}}\right\rangle=-\sum_{i=1}^{n} \sum_{j=1}^{3}\left[\frac{\hbar}{2}\left[\Omega_{i j} S_{i j}^{-}+\text {h.c. }\right]-\Delta_{j} S_{i j}^{+} S_{i j}^{-}\right] \Delta t
$$

and the second order terms with $i=i^{\prime}$

$$
-\frac{1}{\hbar^{2}} \int_{t_{i-1}}^{t_{i}} \mathrm{~d} t^{\prime} \int_{t_{i-1}}^{t^{\prime}} \mathrm{d} t^{\prime \prime} \sum_{i=1}^{n}\left\langle 0_{\mathrm{ph}}\left|H_{\mathrm{I} i}\left(t^{\prime}\right) H_{\mathrm{I} i}\left(t^{\prime \prime}\right)\right| 0_{\mathrm{ph}}\right\rangle=-\sum_{i=1}^{n} \sum_{j=1}^{3}\left[\frac{A_{j}}{2} S_{i j}^{+} S_{i j}^{-}\right] \Delta t
$$

yield contributions corresponding to the sum of $n$ independent atoms. The second order terms with $i \neq i^{\prime}$ represent the dipole-dipole interaction between the atoms. The complete calculation of these terms is given in [44]. It yields

$$
\frac{1}{\hbar^{2}} \int_{t_{i-1}}^{t_{i}} \mathrm{~d} t^{\prime} \int_{t_{i-1}}^{t^{\prime}} \mathrm{d} t^{\prime \prime}\left\langle 0_{\mathrm{ph}}\left|H_{\mathrm{I} k}\left(t^{\prime}\right) H_{\mathrm{Il}}\left(t^{\prime \prime}\right)\right| 0_{\mathrm{ph}}\right\rangle=-\sum_{j=1}^{3} \frac{C_{k l}^{(j)}}{2} S_{k j}^{+} S_{l j}^{-} \Delta t
$$

with the complex dipole-dipole coupling parameters given by

$$
\begin{aligned}
C_{k l}^{(j)}=\frac{3 A_{j}}{2} \mathrm{e}^{\mathrm{i} a_{k l}^{(j)}}[ & \frac{1}{\mathrm{i} a_{k l}^{(j)}}\left(\left(\widehat{\boldsymbol{D}}_{j}^{(k)} \cdot \widehat{\boldsymbol{D}}_{j}^{(l)}\right)-\left(\widehat{\boldsymbol{D}}_{j}^{(k)} \cdot \widehat{\boldsymbol{r}}_{k l}\right)\left(\widehat{\boldsymbol{r}}_{k l} \cdot \widehat{\boldsymbol{D}}_{j}^{(l)}\right)\right) \\
& \left.+\left(\frac{1}{a_{k l}^{(j) 2}}-\frac{1}{\mathrm{i} a_{k l}^{(j) 3}}\right)\left(\left(\widehat{\boldsymbol{D}}_{j}^{(k)} \cdot \widehat{\boldsymbol{D}}_{j}^{(l)}\right)-3\left(\widehat{\boldsymbol{D}}_{j}^{(k)} \cdot \widehat{\boldsymbol{r}}_{k l}\right)\left(\widehat{\boldsymbol{r}}_{k l} \cdot \widehat{\boldsymbol{D}}_{j}^{(l)}\right)\right)\right]
\end{aligned}
$$

where the hats denote the normalized versions of the corresponding vectors. Here the quantity $a_{k l}^{(j)}$ denotes the distance between atoms $k$ and $l$ with respect to the wavelength of the corresponding transition

$$
a_{k l}^{(j)}=\frac{2 \pi r_{k l}}{\lambda_{j}}=\frac{\omega_{j} r_{k l}}{c} .
$$



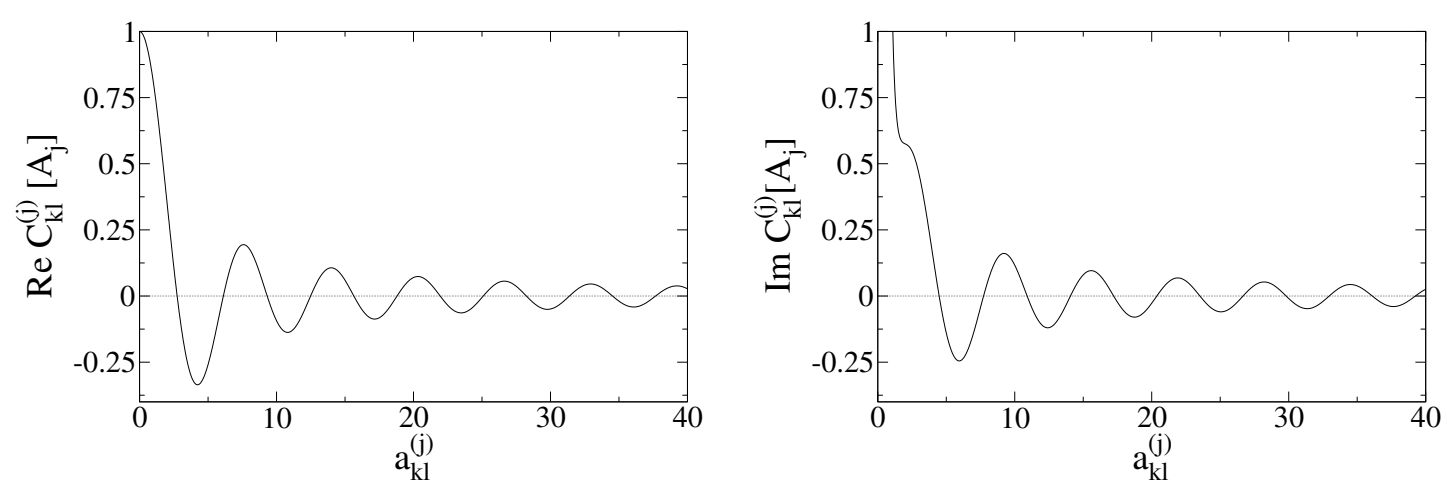

Figure 3.1: Distance dependence of the real and imaginary part of the coupling coefficients $C_{k l}^{(j)}$ with $\vartheta_{k l}=\pi / 2$ following equation (3.11).

In experiments, usually a homogeneous magnetic field is applied to the atoms in order to align the dipole moments. In this case all dipole moments connected with a specific transition are the same $\boldsymbol{D}_{j}^{(i)}=\boldsymbol{D}_{j}$ and the dipole-dipole coupling parameters can be written as

$$
C_{k l}^{(j)}=\frac{3 A_{j}}{2} \mathrm{e}^{\mathrm{i} a_{k l}^{(j)}}\left[\frac{1}{\mathrm{i} a_{k l}^{(j)}}\left(1-\cos ^{2} \vartheta_{k l}\right)+\left(\frac{1}{a_{k l}^{(j) 2}}-\frac{1}{\mathrm{i} a_{k l}^{(j) 3}}\right)\left(1-3 \cos ^{2} \vartheta_{k l}\right)\right],
$$

with the angle $\vartheta_{k l}$ defined as

$$
\cos ^{2} \vartheta_{k l}=\left|\hat{\boldsymbol{D}}_{j} \cdot \hat{\boldsymbol{r}}_{k l}\right|^{2}
$$

which for real dipole moments can be interpreted as the angle between the dipole moment and the line connecting atoms $k$ and $l$. Obviously $C_{k l}^{(j)}=C_{l k}^{(j)}$ in this case. Furthermore the Rabi frequencies $\Omega_{i j}$ now only depend on the atom number via the phase factor $\mathrm{e}^{-\mathrm{i} \boldsymbol{k}_{\mathrm{L}_{j}} \cdot \boldsymbol{r}_{i}}$.

As Figure 3.1 shows, the coupling constants exhibit distance dependent oscillations. These oscillations have a maximum amplitude for $\vartheta_{k l}=\pi / 2$. For $a_{k l}^{(j)} \rightarrow 0$ the imaginary part diverges whereas the real part reaches the value of the corresponding Einstein coefficient.

The conditional Hamiltonian is then given by

$$
\begin{aligned}
H_{\text {cond }}= & \sum_{i=1}^{n} \sum_{j=1}^{3} \frac{\hbar}{2 \mathrm{i}}\left(A_{j}-2 \mathrm{i} \Delta_{3}\right) S_{i j}^{+} S_{i j}^{-}+\sum_{i=1}^{n} \sum_{j=1}^{3} \frac{\hbar}{2}\left[\Omega_{i j} S_{i j}^{-}+\text {h.c. }\right] \\
& +\sum_{\substack{k, l=1 \\
k<l}}^{n} \sum_{j=1}^{3} \frac{\hbar}{2 \mathrm{i}} C_{k l}^{(j)}\left(S_{k j}^{+} S_{l j}^{-}+S_{l j}^{+} S_{k j}^{-}\right) .
\end{aligned}
$$

The special cases for further calculations with a specific level system and a specific number of atoms are given in the respective sections. 


\subsubsection{The reset state}

In addition to the conditional Hamiltonian also the reset state is needed for the following. The situation is more complicated than for a single $\mathrm{V}$ system which makes a calculation necessary. According to equation (2.15) one has to evaluate

$$
\begin{aligned}
\mathscr{R}(\rho) & \Delta t+\mathcal{O}\left(\Delta t^{2}\right) \\
= & \frac{1}{\hbar^{2}} \int_{t}^{t+\Delta t} \mathrm{~d} t^{\prime} \int_{t}^{t+\Delta t} \mathrm{~d} t^{\prime \prime} \sum_{\boldsymbol{k}, \lambda}\left\langle 1_{\boldsymbol{k}, \lambda}\left|H_{\mathrm{I}}\left(t^{\prime}\right)\right| 0_{\mathrm{ph}}\right\rangle \rho(t)\left\langle 0_{\mathrm{ph}}\left|H_{\mathrm{I}}\left(t^{\prime \prime}\right)\right| 1_{\boldsymbol{k}, \lambda}\right\rangle+\mathcal{O}\left(\Delta t^{2}\right) .
\end{aligned}
$$

Analogously to the calculation of the conditional Hamiltonian, there are terms which give the reset state for each atom individually

$$
\begin{aligned}
\frac{1}{\hbar^{2}} \int_{t}^{t+\Delta t} \mathrm{~d} t^{\prime} \int_{t}^{t+\Delta t} \mathrm{~d} t^{\prime \prime} \sum_{\boldsymbol{k}, \lambda}\left\langle 1_{\boldsymbol{k}, \lambda}\left|H_{\mathrm{I} i}\left(t^{\prime}\right)\right| 0_{\mathrm{ph}}\right\rangle \rho\left\langle 0_{\mathrm{ph}}\left|H_{\mathrm{I} i}\left(t^{\prime \prime}\right)\right| 1_{\boldsymbol{k}, \lambda}\right\rangle & \\
& =A_{j} S_{i j}^{-} \rho S_{i j}^{+} \Delta t+\mathcal{O}\left(\Delta t^{2}\right)
\end{aligned}
$$

and terms which correspond to the dipole-dipole interaction between a pair of atoms

$$
\begin{aligned}
\frac{1}{\hbar^{2}} \int_{t}^{t+\Delta t} \mathrm{~d} t^{\prime} \int_{t}^{t+\Delta t} \mathrm{~d} t^{\prime \prime} \sum_{\boldsymbol{k}, \lambda}\left\langle 1_{\boldsymbol{k}, \lambda}\left|H_{\mathrm{I} k}\left(t^{\prime}\right)\right| 0_{\mathrm{ph}}\right\rangle \rho\left\langle 0_{\mathrm{ph}}\right| & H_{\mathrm{I} l}\left(t^{\prime \prime}\right)\left|1_{\boldsymbol{k}, \lambda}\right\rangle \\
& =\operatorname{Re} C_{k l}^{(j)} S_{k j}^{-} \rho S_{l j}^{+} \Delta t+\mathcal{O}\left(\Delta t^{2}\right) .
\end{aligned}
$$

As above, equal dipole moments $\boldsymbol{D}_{j}^{(i)}=\boldsymbol{D}_{j}$ have been assumed. This leads to the reset state

$$
\mathscr{R}(\rho)=\sum_{i=1}^{n} \sum_{j=1}^{3} A_{j} S_{i j}^{-} \rho S_{i j}^{+}+\sum_{\substack{k, l=1 \\ k<l}}^{n} \sum_{j=1}^{3} \operatorname{Re} C_{k l}^{(j)}\left(S_{k j}^{-} \rho S_{l j}^{+}+S_{l j}^{-} \rho S_{k j}^{+}\right) .
$$

For the full calculation Reference [44] can be consulted.

\subsection{Two dipole-interacting atoms}

In this section cooperative effects of two dipole-interacting atoms are investigated. First the necessary methods are presented for two V systems. Afterwards two threelevel systems in a D configuration modeling the level scheme of $\mathrm{Hg}^{+}$-ions [23] and two four-level systems for the experiments with $\mathrm{Ba}^{+}$-ions $[3,19,20]$ are studied. 


\subsubsection{Two V systems}

The conditional Hamiltonian for two dipole-interacting $\mathrm{V}$ systems can be read off from equation (3.13) by setting $n=2, \Omega_{1}=0$, and $A_{1}=A_{2}=0$. For $\Delta_{2}=0$, this results in

$$
\begin{aligned}
H_{\text {cond }}= & \frac{\hbar}{2 \mathrm{i}}\left[\left(A_{3}-2 \mathrm{i} \Delta_{3}\right)\left[S_{13}^{+} S_{13}^{-}+S_{23}^{+} S_{23}^{-}\right]+C_{3}\left[S_{13}^{+} S_{23}^{-}+S_{23}^{+} S_{13}^{-}\right]\right] \\
& +\sum_{i=1}^{2} \sum_{j=2}^{3} \frac{\hbar}{2}\left[\Omega_{i j} S_{i j}^{-}+\text {h.c. }\right]
\end{aligned}
$$

with $C_{3}=C_{12}^{(3)}=C_{21}^{(3)}$. For further simplification it is assumed in the following that the lasers are directed orthogonal to the line connecting the two atoms. Then the Rabi frequencies connected with a specific laser are equal and can be assumed to be real

$$
\Omega_{13}=\Omega_{23}=\Omega_{3}, \quad \Omega_{12}=\Omega_{22}=\Omega_{2} .
$$

With the same assumptions the reset state of equation (3.17) becomes

$$
\mathscr{R}(\rho)=A_{3}\left[S_{13}^{-} \rho S_{13}^{+}+S_{23}^{-} \rho S_{23}^{+}\right]+\operatorname{Re} C_{3}\left[S_{13}^{-} \rho S_{23}^{+}+S_{23}^{-} \rho S_{13}^{+}\right] .
$$

For a single $\mathrm{V}$ system the reset state was a density matrix of a pure state. This has the advantage that a single fluorescence trajectory can always be described by a pure state. For two interacting $\mathrm{V}$ systems this is not possible anymore. However, with the reset operators

$$
R_{+}:=\left(S_{13}^{-}+S_{23}^{-}\right) / \sqrt{2}, \quad \quad R_{-}:=\left(S_{13}^{-}-S_{23}^{-}\right) / \sqrt{2}
$$

it is possible to write the reset state as a sum of two density matrices of pure states

$$
\mathscr{R}(\rho)=\left(A_{3}+\operatorname{Re} C_{3}\right) R_{+} \rho R_{+}^{\dagger}+\left(A_{3}-\operatorname{Re} C_{3}\right) R_{-} \rho R_{-}^{\dagger} .
$$

\section{Dicke basis}

For further calculations it is convenient to use instead of the basis of product states a basis which is adapted to the symmetry of the system under the exchange of the atoms. This so-called Dicke basis is given by

$$
|g\rangle=|1\rangle|1\rangle, \quad\left|e_{2}\right\rangle=|2\rangle|2\rangle, \quad\left|e_{3}\right\rangle=|3\rangle|3\rangle,
$$

and

$$
\left|s_{i j}\right\rangle=(|i\rangle|j\rangle+|j\rangle|i\rangle) / \sqrt{2}, \quad\left|a_{i j}\right\rangle=(|i\rangle|j\rangle-|j\rangle|i\rangle) / \sqrt{2}
$$




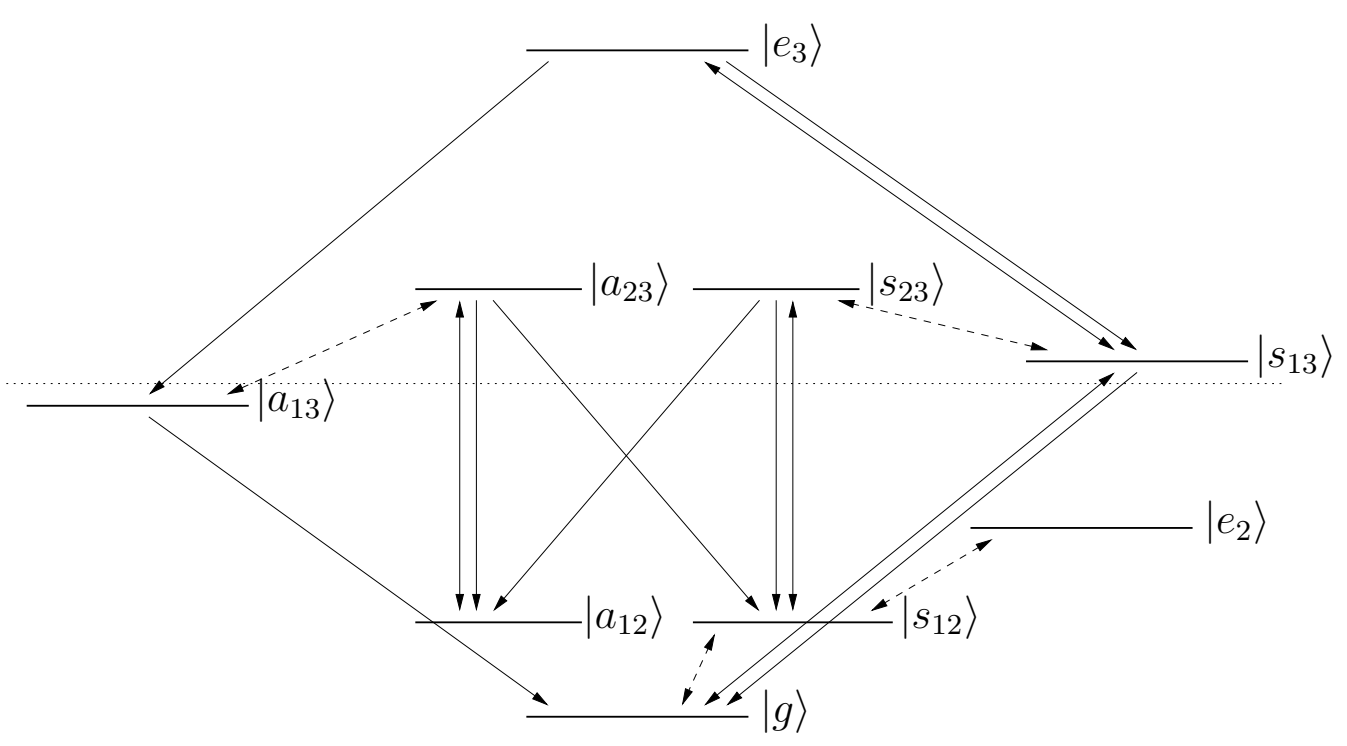

Figure 3.2: Level scheme of two $V$ systems in Dicke basis. Arrows with solid lines represent decays with $A_{3} \pm R e C_{3}$, solid lined double-sided arrows represent laser coupling with $\Omega_{3}$, dashed double-sided represent laser coupling with $\Omega_{2}$. The levels $\left|s_{13}\right\rangle$ and $\left|a_{13}\right\rangle$ are shifted by $\pm \operatorname{Im} C_{3}$ with respect to their energy without interaction (dotted line).

In this basis the conditional Hamiltonian takes the form

$$
\begin{aligned}
H_{\text {cond }}= & \frac{\hbar}{2 \mathrm{i}}\left(A_{3}-2 \mathrm{i} \Delta_{3}\right)\left[2\left|e_{3}\right\rangle\left\langle e_{3}|+| s_{23}\right\rangle\left\langle s_{23}|+| a_{23}\right\rangle\left\langle a_{23}|+| s_{13}\right\rangle\left\langle s_{13}|+| a_{13}\right\rangle\left\langle a_{13}\right|\right] \\
& +\frac{\hbar}{2 \mathrm{i}} C_{3}\left[\left|s_{13}\right\rangle\left\langle s_{13}|-| a_{13}\right\rangle\left\langle a_{13}\right|\right] \\
& +\frac{\hbar}{2} \Omega_{3}\left[\sqrt{2}\left(|g\rangle\left\langle s_{13}|+| s_{13}\right\rangle\left\langle e_{3}\right|\right)+\left|s_{12}\right\rangle\left\langle s_{23}|-| a_{12}\right\rangle\left\langle a_{23}\right|+\text { h.c. }\right] \\
& +\frac{\hbar}{2} \Omega_{2}\left[\sqrt{2}\left(|g\rangle\left\langle s_{12}|+| s_{12}\right\rangle\left\langle e_{2}\right|\right)+\left|s_{13}\right\rangle\left\langle s_{23}|+| a_{13}\right\rangle\left\langle a_{23}\right|+\text { h.c. }\right]
\end{aligned}
$$

and the reset operators of (3.21) are given by

$$
\begin{aligned}
& R_{+}=|g\rangle\left\langle s_{13}|+| s_{13}\right\rangle\left\langle e_{3}\right|+\left(\left|s_{12}\right\rangle\left\langle s_{23}|-| a_{12}\right\rangle\left\langle a_{23}\right|\right) / \sqrt{2}, \\
& R_{-}=|g\rangle\left\langle a_{13}|+| a_{13}\right\rangle\left\langle e_{3}\right|+\left(\left|s_{12}\right\rangle\left\langle a_{23}|+| a_{12}\right\rangle\left\langle s_{23}\right|\right) / \sqrt{2} .
\end{aligned}
$$

Figure 3.2 shows the level scheme arising from the conditional Hamiltonian and the reset state in Dicke basis. The coupling between the atoms due to the dipoledipole interaction has two distinguished effects on this level scheme. On the one hand the energy of the states $\left|s_{13}\right\rangle$ and $\left|a_{13}\right\rangle$ is shifted by $\pm \operatorname{Im} C_{3}$, respectively. On the other hand the decay rate of transitions between states with the same symmetry is enhanced by $\operatorname{Re} C_{3}$ whereas it is reduced by $\operatorname{Re} C_{3}$ for transitions between states of 
different symmetry. Furthermore, one can see that the lasers only couple states with the same symmetry. This arises from the assumption that the lasers are directed perpendicular to the axis of the atoms.

\section{Transition rates via Bloch equations}

In contrast to a single $\mathrm{V}$ system, the reset state of two such systems is not independent of the state before the emission of a photon. This is due to the fact that there are many different possible transitions on which the decay may occur, as can be seen in figure 3.2. Therefore it is much more difficult to apply the quantum jump approach for the calculation of the transition rates. Therefore another method is used here which is based on a perturbation theoretical approach applied to the Bloch equations of this system [17]. This method will be presented in this section.

In principal, a system of two atoms which exhibit bright and dark periods in their fluorescence should show three periods of different intensity. A dark period $I_{0}$ in which both atoms do not emit any photons, a period $I_{1}$ with the intensity of the bright period of a single atom where one atom is dark and the other one emits photons, and a period of double intensity $I_{2}$ in which both atoms emit photons. If the weak Rabi frequency is neglected the system decouples into three independent subsystems as can be seen in Figure 3.2

$$
\begin{aligned}
& \mathscr{S}_{0}=\left\{\left|e_{2}\right\rangle\right\}, \\
& \mathscr{S}_{1}=\left\{\left|s_{12}\right\rangle,\left|a_{12}\right\rangle,\left|s_{23}\right\rangle,\left|a_{23}\right\rangle\right\}, \\
& \mathscr{S}_{2}=\left\{|g\rangle,\left|s_{13}\right\rangle,\left|a_{13}\right\rangle,\left|e_{3}\right\rangle\right\} .
\end{aligned}
$$

As in the one atom case where one can assume to good approximation that the population is mainly in state $|2\rangle$ in a dark period and mostly in $\{|1\rangle,|3\rangle\}$ in a bright period, the different subspaces $\mathscr{S}_{0}, \mathscr{S}_{1}, \mathscr{S}_{2}$ can be associated with the different intensity periods $I_{0}, I_{1}, I_{2}$. The transition rates will thus be calculated by starting with a density matrix in one particular subspace. Then the rate of build-up of population in the other subspaces will be determined. Taking a density matrix $\rho_{0, i} \in \mathscr{S}_{i}$ at a time $t_{0}$ the state after a time $t_{0}+\Delta t$ is calculated in perturbation theory with respect to the small parameters. The time interval $\Delta t$ used here should be long in comparison to the mean time between the emission of two photons but short compared to the duration of the intensity periods

$$
\Omega_{3}^{-1}, A_{3}^{-1} \ll \Delta t \ll \Omega_{2}^{-1} .
$$

For the calculation the Bloch equation is written in a Liouvillean form

$$
\dot{\rho}=\mathscr{L} \rho=\left\{\mathscr{L}_{0}\left(A_{3}, C_{3}, \Omega_{3}, \Delta_{3}\right)+\mathscr{L}_{1}\left(\Omega_{2}\right)\right\} \rho,
$$

where $\mathscr{L}_{1}$ serves as the perturbation. For the state at time $t+\Delta t$ one then has to first order in perturbation theory

$$
\rho\left(t_{0}+\Delta t ; \rho_{0, i}\right)=\mathrm{e}^{\mathscr{L}_{0} \Delta t} \rho_{0, i}+\int_{0}^{\Delta t} \mathrm{~d} \tau \mathrm{e}^{\mathscr{L}_{0}(\Delta t-\tau)} \mathscr{L}_{1} \mathrm{e}^{\mathscr{L}_{0} \tau} \rho_{0, i} .
$$


Since $\Delta t$ satisfies the condition (3.27), the first term on the right hand side gives the equilibrium state $\rho_{\mathrm{ss}, i}$ of $\mathscr{L}_{1}$ in subsystem $\mathscr{S}_{i}$. The term $\mathrm{e}^{\mathscr{L}_{0} \tau} \rho_{0, i}$ under the integrand also rapidly reaches this equilibrium state. After the substitution $\tau \rightarrow \Delta t-\tau$ one therefore has

$$
\rho\left(t_{0}+\Delta t ; \rho_{0, i}\right)=\rho_{\mathrm{ss}, i}+\int_{0}^{\Delta t} \mathrm{~d} \tau \mathrm{e}^{\mathscr{L}_{0} \tau} \mathscr{L}_{1} \rho_{\mathrm{ss}, i}
$$

Since for two $\mathrm{V}$ system $\mathscr{L}_{1}$ corresponds to the driving with the weak laser, the term $\mathscr{L}_{1} \rho_{\mathrm{ss}, i}$ only consists of coherences between subspace $\mathscr{S}_{i}$ and the neighboring subspaces. The zero-eigenvalue subspace of $\mathscr{L}_{0}$, on the other hand, is spanned by the quasi-steady states $\rho_{\mathrm{ss}, i}$. Therefore, $\mathscr{L}_{1} \rho_{\mathrm{ss}, i}$ has no components in the zero eigenvalue subspace of $\mathscr{L}_{0}$. The other eigenvalues all have negative real parts of the order of $A_{3}$ and $\Omega_{3}$. Therefore the integrand in equation (3.30) is rapidly damped which allows to extend the upper integration limit to infinity. This yields

$$
\rho\left(t_{0}+\Delta t ; \rho_{0, i}\right)=\rho_{\mathrm{ss}, i}+\left(\epsilon-\mathscr{L}_{0}\right)^{-1} \mathscr{L}_{1} \rho_{\mathrm{ss}, i}
$$

independent of $\Delta t$ [17], where the limit $\epsilon \searrow 0$ is implied.

Let $\left|x_{i}\right\rangle, i=1 \ldots 9$ denote a numbering of the Dicke states. From the Bloch equations one gets the exact relations

$$
\begin{aligned}
\frac{\mathrm{d}}{\mathrm{d} t}\left\langle e_{2}|\rho| e_{2}\right\rangle & =\sqrt{2} \Omega_{2} \operatorname{Im}\left\langle s_{12}|\rho| e_{2}\right\rangle, \\
\frac{\mathrm{d}}{\mathrm{d} t} \sum_{x_{i} \in \mathscr{S}_{2}}\left\langle x_{i}|\rho| x_{i}\right\rangle & =\Omega_{2} \operatorname{Im}\left[\sqrt{2}\left\langle s_{12}|\rho| g\right\rangle+\left\langle s_{23}|\rho| s_{13}\right\rangle+\left\langle a_{23}|\rho| a_{13}\right\rangle\right], \\
\frac{\mathrm{d}}{\mathrm{d} t} \sum_{x_{i} \in \mathscr{S}_{1}}\left\langle x_{i}|\rho| x_{i}\right\rangle & =-\frac{\mathrm{d}}{\mathrm{d} t}\left[\left\langle e_{2}|\rho| e_{2}\right\rangle+\sum_{x_{i} \in \mathscr{S}_{2}}\left\langle x_{i}|\rho| x_{i}\right\rangle\right] .
\end{aligned}
$$

Together with equation (3.31) this allows to calculate the transition rates as

$$
p_{i j}=\left.\frac{\mathrm{d}}{\mathrm{d} t} \sum_{x_{k} \in \mathscr{S}_{j}}\left\langle x_{k}|\rho| x_{k}\right\rangle\right|_{\rho=\rho\left(t_{0}+\Delta t ; \rho_{0, i}\right)} .
$$

Note that $p_{02}=p_{20}=0$ so that no direct, i.e. instantaneous, double jumps occur.

\section{Transition rates}

The stationary states in the different subsystems are determined by $\mathscr{L}_{0} \rho_{\mathrm{ss}, i}=0$, where $\mathscr{L}_{0}$ is restricted to the subspace $\left\{\left|x_{k}\right\rangle\left\langle x_{k}\right|\right\},\left|x_{k}\right\rangle \in \mathscr{S}_{i}$. Since $\mathscr{S}_{0}$ is onedimensional, the corresponding stationary state is simply given by

$$
\rho_{\mathrm{ss}, 0}=\left|e_{2}\right\rangle\left\langle e_{2}\right|
$$


By symmetry the steady state for $\mathscr{S}_{1}$ is given by

$$
\begin{aligned}
\rho_{\mathrm{ss}, 1}= & \frac{1}{2}\left[\rho_{\mathrm{ss}}^{2 \mathrm{~N}} \otimes|2\rangle\langle 2|+| 2\rangle\langle 2| \otimes \rho_{\mathrm{ss}}^{2 \mathrm{~N}}\right] \\
= & \frac{1}{2} \frac{A_{3}^{2}+\Omega_{3}^{2}+4 \Delta_{3}^{2}}{A_{3}^{2}+2 \Omega_{3}^{2}+4 \Delta_{3}^{2}}\left(\left|s_{12}\right\rangle\left\langle s_{12}|+| a_{12}\right\rangle\left\langle a_{12}\right|\right) \\
& +\frac{1}{2} \frac{\Omega_{3}^{2}}{A_{3}^{2}+2 \Omega_{3}^{2}+4 \Delta_{3}^{2}}\left(\left|s_{23}\right\rangle\left\langle s_{23}|+| a_{23}\right\rangle\left\langle a_{23}\right|\right) \\
& +\left\{\frac{1}{2} \frac{\left(\mathrm{i} A_{3}+2 \Delta_{3}\right) \Omega_{3}}{A_{3}^{2}+2 \Omega_{3}^{2}+4 \Delta_{3}^{2}}\left(\left|s_{12}\right\rangle\left\langle s_{23}|-| a_{12}\right\rangle\left\langle a_{23}\right|\right)+\text { h.c. }\right\},
\end{aligned}
$$

independent of $C_{3}$. For subspace $\mathscr{S}_{2}$ one calculates

$$
\begin{aligned}
\rho_{\mathrm{ss}, 2}=\frac{1}{N}[ & \left\{\left(A_{3}^{2}+\Omega_{3}^{2}+4 \Delta_{3}^{2}\right)^{2}+\left(A_{3}^{2}+4 \Delta_{3}^{2}\right)\left(\left|C_{3}\right|^{2}+2 A_{3} \operatorname{Re} C_{3}-4 \Delta_{3} \operatorname{Im} C_{3}\right)\right\}|g\rangle\langle g| \\
& +\Omega_{3}^{2}\left(2 A_{3}^{2}+\Omega_{3}^{2}+8 \Delta_{3}^{2}\right)\left|s_{13}\right\rangle\left\langle s_{13}\right|+\Omega_{3}^{4}\left\{\left|e_{3}\right\rangle\left\langle e_{3}|+| a_{13}\right\rangle\left\langle a_{13}\right|\right\} \\
+ & \left\{\Omega _ { 3 } ( \mathrm { i } A _ { 3 } + 2 \Delta _ { 3 } ) \left[\sqrt{2}\left(A_{3}^{2}+\Omega_{3}^{2}+4 \Delta_{3}^{2}\left(A_{3}+2 \mathrm{i} \Delta_{3}\right) C_{3}\right)|g\rangle\left\langle s_{13}\right|\right.\right. \\
& \left.\left.+\mathrm{i} \Omega_{3}\left(A_{3}+C_{3}-2 \mathrm{i} \Delta_{3}\right)|g\rangle\left\langle e_{3}\left|+\sqrt{2} \Omega_{3}^{2}\right| s_{13}\right\rangle\left\langle e_{3}\right|+\text { h.c. }\right\}\right]
\end{aligned}
$$

with

$$
N=\left(A_{3}^{2}+2 \Omega_{3}^{2}+4 \Delta_{3}^{2}\right)^{2}+\left(A_{3}^{2}+4 \Delta_{3}^{2}\right)\left(\left|C_{3}\right|^{2}+2 A_{3} \operatorname{Re} C_{3}-4 \Delta_{3} \operatorname{Im} C_{3}\right) .
$$

The transition rates for the $\mathrm{V}$ systems can now be calculated according to equations (3.31), (3.32), and (3.33) using these steady states. The result is

$$
\begin{aligned}
p_{01}= & 2 \frac{A_{3} \Omega_{2}^{2}}{\Omega_{3}^{2}}, \\
p_{10}= & \frac{A_{3}^{3} \Omega_{2}^{2}}{\Omega_{3}^{2}\left(A_{3}^{2}+2 \Omega_{3}^{2}+4 \Delta_{3}^{2}\right)}, \\
p_{12}= & \frac{A_{3} \Omega_{2}^{2}}{\Omega_{3}^{2}}\left[1+2 \operatorname{Re} C_{3} \frac{A_{3}}{A_{3}^{2}+2 \Omega_{3}^{2}+4 \Delta_{3}^{2}}-4 \operatorname{Im} C_{3} \frac{A_{3}}{A_{3}^{2}+2 \Omega_{3}^{2}+4 \Delta_{3}^{2}}\right], \\
p_{21}=2 \frac{A_{3} \Omega_{2}^{2}\left(A_{3}^{2}+4 \Delta_{3}^{2}\right)}{\Omega_{3}^{2}\left(A_{3}^{2}+2 \Omega_{3}^{2}+4 \Delta_{3}^{2}\right)}\left[1+2 \operatorname{Re} C_{3} \frac{A_{3}\left(A_{3}^{2}+4 \Omega_{3}^{2}+4 \Delta_{3}^{2}\right)}{\left(A_{3}^{2}+2 \Omega_{3}^{2}+4 \Delta_{3}^{2}\right)^{2}}\right. & \left.\quad-4 \operatorname{Im} C_{3} \frac{\Delta_{3}\left(A_{3}^{2}+4 \Omega_{3}^{2}+4 \Delta_{3}^{2}\right)}{\left(A_{3}^{2}+2 \Omega_{3}^{2}+4 \Delta_{3}^{2}\right)^{2}}\right],
\end{aligned}
$$

to first order in $C_{3}$. For $p_{01}$ and $p_{10}$ this is also the exact result, to all orders in $C_{3}$. For the other two transition rates exact expressions in $C_{3}$ can in principal be evaluated, but will not be given here since they are too complicated. For usual 


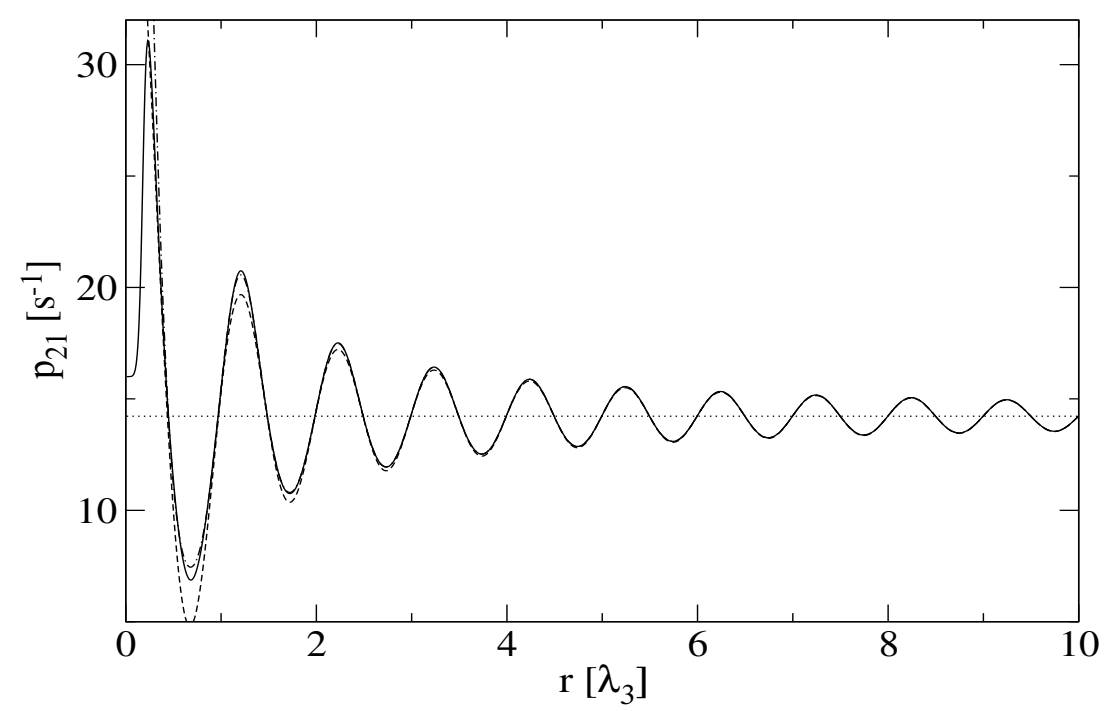

Figure 3.3: Transition rate $p_{21}$ for two dipole-interacting $V$ systems. Dotted line: independent atoms. Dashed line: up to first order. Dash-dotted line: up to second order. Solid line: exact expression. Parameter values: $A_{3}=2 \times 10^{8} \mathrm{~s}^{-1}, \Omega_{3}=$ $5 \times 10^{7} s^{-1}, \Omega_{2}=10^{4} s^{-1}$.

parameter values the second order corrections are small at distances of more than a wavelength $\lambda_{3}[17]$.

As can be seen from equations (3.35) the cooperative effects are maximal if $\Omega_{3}$ is small compared to $A_{3}$, i.e. for weak driving of the strong transition. In Figure 3.3 this case is illustrated for the rate $p_{21}$. At a distance of one wavelength of the strong transition deviation from the rate without interaction is about $40 \%$. At usual experimental distances of about $10 \lambda_{3}$ the cooperative effects have decreased to around $5 \%$.

\section{The double jump rate}

The physical quantity investigated in the experiments of References [19, 20, 23, 22, 27 is the double jump rate. This is the rate at which jumps between periods of intensities that differ by twice the intensity of a single system occur within a small time interval $T_{\mathrm{W}}$, which cannot be resolved experimentally. It can be expressed through the transition rates $p_{i j}$ as follows [17].

The full double jump rate $n_{\mathrm{DJ}}$ is a sum of the rate for upward and downward double jumps

$$
n_{\mathrm{DJ}}=n_{\mathrm{DJ}}^{20}+n_{\mathrm{DJ}}^{02} .
$$

First the downward rate is calculated. Each period of double intensity $I_{2}$ is followed by one with single intensity $I_{1}$. The probability that the latter period is shorter than 


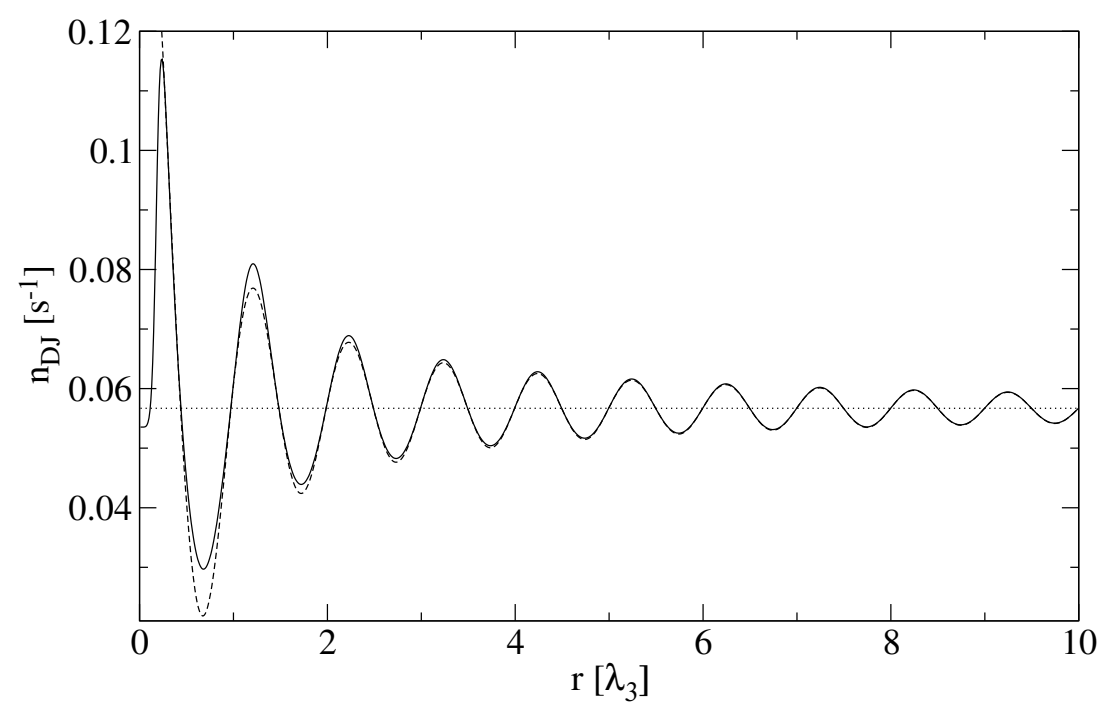

Figure 3.4: Double jump rate $n_{D J}$ for two dipole-interacting V systems. Dotted line: independent atoms. Dashed line: up to first order. Solid line: exact expression. Parameter values: $T_{W}=10^{-3} \mathrm{~s}$, other parameters like in Figure 3.3.

$T_{\mathrm{W}}$ is given by

$$
p_{T_{1}<T_{\mathrm{W}}}=1-\mathrm{e}^{-\left(p_{10}+p_{12}\right) T_{\mathrm{W}}} .
$$

The branching ratio for the following period to be of zero intensity is $p_{10} /\left(p_{10}+p_{12}\right)$. With the mean number of intensity periods $I_{i}$ per unit time denoted by $n_{i}$ the rate $n_{\mathrm{DJ}}^{02}$ is given by

$$
n_{\mathrm{DJ}}^{20}=n_{2} \frac{p_{10}}{p_{10}+p_{12}}\left(1-\mathrm{e}^{-\left(p_{10}+p_{12}\right) T_{\mathrm{W}}}\right) .
$$

Analogously, one finds

$$
n_{\mathrm{DJ}}^{02}=n_{0} \frac{p_{12}}{p_{10}+p_{12}}\left(1-\mathrm{e}^{-\left(p_{10}+p_{12}\right) T_{\mathrm{W}}}\right) .
$$

It remains to determine $n_{0}$ and $n_{2}$. Since a period of intensity $I_{1}$ is either followed by a period of intensity $I_{0}$ or by a period of intensity $I_{2}$ one has, with the respective branching ratios

$$
n_{0}=\frac{p_{10}}{p_{10}+p_{12}} n_{1}, \quad n_{2}=\frac{p_{12}}{p_{10}+p_{12}} n_{1} .
$$

Thus $n_{\mathrm{DJ}}^{20}$ and $n_{\mathrm{DJ}}^{02}$ are equal and the total double jump rate is given by

$$
n_{\mathrm{DJ}}=2 n_{1} \frac{p_{10} p_{12}}{\left(p_{10}+p_{12}\right)^{2}}\left(1-\mathrm{e}^{-\left(p_{10}+p_{12}\right) T_{\mathrm{W}}}\right) .
$$

The mean durations of the intensity periods $T_{i}$ are given by

$$
T_{0}=\frac{1}{p_{01}}, \quad T_{1}=\frac{1}{p_{10}+p_{12}}, \quad T_{2}=\frac{1}{p_{21}} .
$$


In addition they fulfill

$$
\sum_{i=0}^{2} n_{i} T_{i}=1
$$

which leads to

$$
n_{1}=\frac{p_{01} p_{21}\left(p_{10}+p_{12}\right)}{p_{01} p_{12}+p_{01} p_{21}+p_{10} p_{21}} .
$$

For $T_{\mathrm{W}} \ll T_{1}$ the exponential can be expanded, finally leading to

$$
n_{\mathrm{DJ}}=2 \frac{p_{01} p_{10} p_{12} p_{21}}{p_{01} p_{12}+p_{01} p_{21}+p_{10} p_{21}} T_{\mathrm{W}} .
$$

Figure 3.4 shows the double jump rate for parameter values maximizing cooperative effects. The results are similar to those of the transition rate $p_{21}$.

\subsubsection{Two D systems}

The D configuration, as displayed in Figure 2.2, can be seen as a model of the level system of $\mathrm{Hg}^{+}$used in the experiments of References [23, 45]. In the following two dipole-interacting $\mathrm{D}$ systems will be investigated. The transition rates between the three different intensity periods and the double jump rate will be calculated. Due to the incoherent population of the dark state $|2\rangle$ the method presented for the $\mathrm{V}$ systems has to be modified.

\section{Conditional Hamiltonian and reset state}

Using the Dicke basis of equation (3.23) the conditional Hamiltonian for two dipoleinteracting D systems can be written as

$$
\begin{aligned}
H_{\text {cond }}= & \frac{\hbar}{2 \mathrm{i}}\left\{A_{1}\left[2\left|e_{2}\right\rangle\left\langle e_{2}\left|+\sum_{j=1}^{2}\right| s_{j j+1}\right\rangle\left\langle s_{j j+1}|+| a_{j j+1}\right\rangle\left\langle a_{j j+1}\right|\right]\right. \\
& +\left(A_{2}+A_{3}-2 \mathrm{i} \Delta_{3}\right)\left[2\left|e_{3}\right\rangle\left\langle e_{3}\left|+\sum_{j=1}^{2}\right| s_{j 3}\right\rangle\left\langle s_{j 3}|+| a_{j 3}\right\rangle\left\langle a_{j 3}\right|\right] \\
& \left.+\sum_{j=1}^{2} C_{j}\left[\left|s_{j j+1}\right\rangle\left\langle s_{j j+1}|-| a_{j j+1}\right\rangle\left\langle a_{j j+1}\right|\right]+C_{3}\left[\left|s_{13}\right\rangle\left\langle s_{13}|-| a_{13}\right\rangle\left\langle a_{13}\right|\right]\right\} \\
& +\frac{\hbar}{2} \Omega_{3}\left\{\sqrt{2}\left(|g\rangle\left\langle s_{13}|+| s_{13}\right\rangle\left\langle e_{3}\right|\right)+\left|s_{12}\right\rangle\left\langle s_{23}|-| a_{12}\right\rangle\left\langle a_{23}\right|+\text { h.c. }\right\} .
\end{aligned}
$$

The reset state is given by

$$
\mathscr{R}(\rho)=\sum_{j=1}^{3}\left[\left(A_{j}+\operatorname{Re} C_{j}\right) R_{+}^{(j)} \rho R_{+}^{(j)^{\dagger}}+\left(A_{j}-\operatorname{Re} C_{j}\right) R_{-}^{(j)} \rho R_{-}^{(j)^{\dagger}}\right],
$$




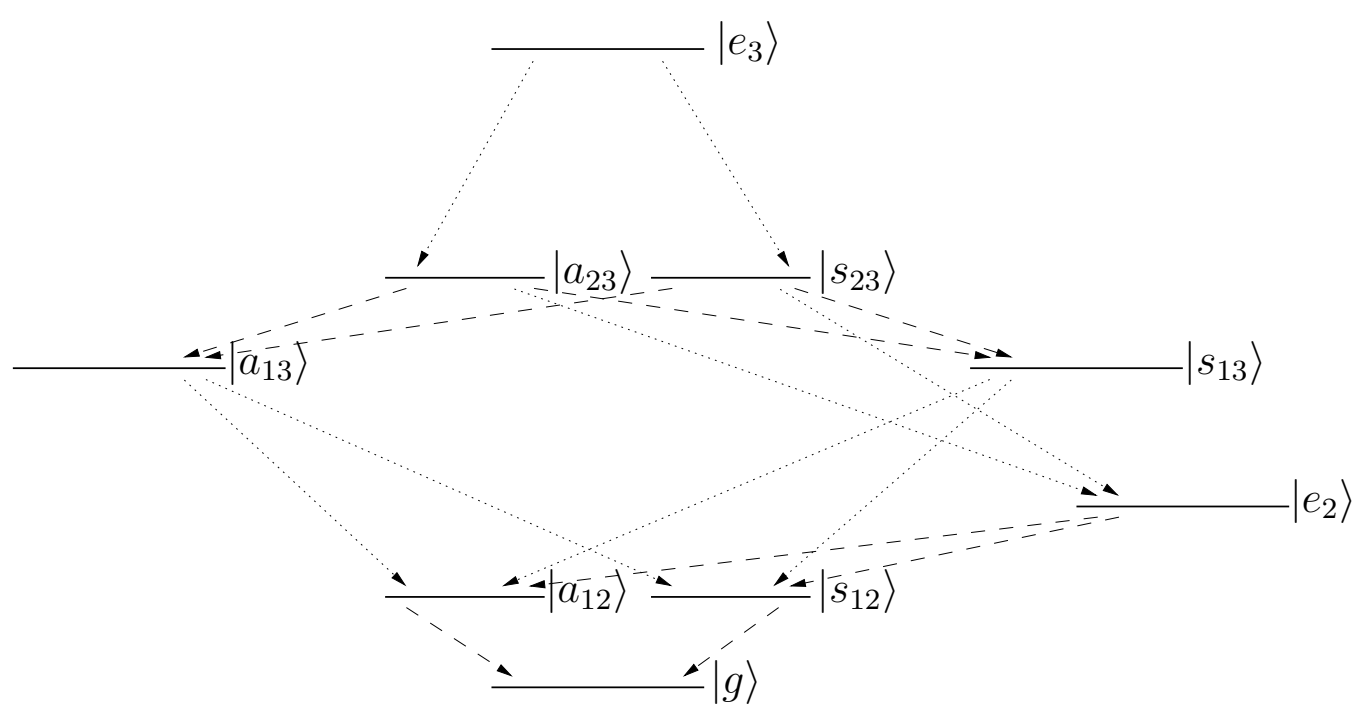

Figure 3.5: Level configuration of two D systems in the Dicke basis. Only the weak transitions with the rates $A_{2} \pm R e C_{2}$ (dotted arrows) and $A_{1} \pm R e C_{1}$ (dashed arrows). Line shifts due to detuning and to $\operatorname{Im} C_{i}$ are omitted.

where the reset operators

$$
\begin{aligned}
& R_{+}^{(1)}=|g\rangle\left\langle s_{12}|+| s_{12}\right\rangle\left\langle e_{2}\right|+\frac{1}{\sqrt{2}}\left(\left|s_{13}\right\rangle\left\langle s_{23}|+| a_{13}\right\rangle\left\langle a_{23}\right|\right), \\
& R_{-}^{(1)}=|g\rangle\left\langle a_{12}|+| a_{12}\right\rangle\left\langle e_{2}\right|+\frac{1}{\sqrt{2}}\left(\left|a_{13}\right\rangle\left\langle s_{23}|-| s_{13}\right\rangle\left\langle a_{23}\right|\right), \\
& R_{+}^{(2)}=\left|e_{2}\right\rangle\left\langle s_{23}|+| s_{23}\right\rangle\left\langle e_{3}\right|+\frac{1}{\sqrt{2}}\left(\left|s_{12}\right\rangle\left\langle s_{13}|+| a_{12}\right\rangle\left\langle a_{13}\right|\right), \\
& R_{-}^{(2)}=\left|e_{2}\right\rangle\left\langle a_{23}|+| a_{23}\right\rangle\left\langle e_{3}\right|+\frac{1}{\sqrt{2}}\left(\left|s_{12}\right\rangle\left\langle a_{13}|-| a_{12}\right\rangle\left\langle s_{13}\right|\right), \\
& R_{+}^{(3)}=|g\rangle\left\langle s_{13}|+| s_{13}\right\rangle\left\langle e_{3}\right|+\frac{1}{\sqrt{2}}\left(\left|s_{12}\right\rangle\left\langle s_{23}|-| a_{12}\right\rangle\left\langle a_{23}\right|\right), \\
& R_{-}^{(3)}=|g\rangle\left\langle a_{13}|+| a_{13}\right\rangle\left\langle e_{3}\right|+\frac{1}{\sqrt{2}}\left(\left|s_{12}\right\rangle\left\langle a_{23}|+| a_{12}\right\rangle\left\langle s_{23}\right|\right) .
\end{aligned}
$$

have been used. As one can see in Figure 3.5 the level scheme without the weak parameters $A_{1}, A_{2}$ is identical to the corresponding level scheme for two $\mathrm{V}$ systems. Accordingly, one has the same independent subspaces $\mathscr{S}_{0}, \mathscr{S}_{1}, \mathscr{S}_{2}$ given by equation (3.26). The transitions between the subsystems occur via spontaneous decay in this case. The corresponding rates are given by $A_{1} \pm \operatorname{Re} C_{1}$ for the transition to a period with higher intensity and with $A_{2} \pm \operatorname{Re} C_{2}$ for a transition to a period with lower intensity. 


\section{Transition rates}

For the calculation of the transition rates a perturbation theoretical approach similar to that for the $\mathrm{V}$ systems can be used. The Bloch equation is written in a Liouvillean form as in the $\mathrm{V}$ system case

$$
\begin{aligned}
\dot{\rho} & =-\frac{\mathrm{i}}{\hbar}\left[H_{\text {cond }} \rho-\rho H_{\text {cond }}^{\dagger}\right]+\mathscr{R}(\rho) \\
& =\left\{\mathscr{L}_{0}\left(A_{3}, C_{3}, \Omega_{3}, \Delta_{3}\right)+\mathscr{L}_{1}\left(A_{1}, A_{2}, C_{1}, C_{2}\right)\right\} \rho .
\end{aligned}
$$

The perturbation part of the Liouvillean depends on the weak decay rates and the corresponding dipole-dipole interaction parameters. For a given state $\rho_{0, i}$ in one of the subsystems at time $t_{0}$ the resulting state at time $t+\Delta t$ can again be calculated in perturbation theory to first order by

$$
\rho\left(t_{0}+\Delta t ; \rho_{0, i}\right)=\rho_{\mathrm{ss}, i}+\int_{0}^{\Delta t} \mathrm{~d} \tau \mathrm{e}^{\mathscr{L}_{0} \tau} \mathscr{L}_{1} \rho_{\mathrm{ss}, i},
$$

where the time interval $\Delta t$ has to fulfill the condition

$$
A_{3}^{-1}, \Omega_{3}^{-1} \ll \Delta t \ll A_{1}^{-1}, A_{2}^{-1} .
$$

While $\mathscr{L}_{1} \rho_{\mathrm{ss}, i}$ is a superposition of just the eigenstates for nonzero eigenvalues of $\mathscr{L}_{0}$ in the case of the $\mathrm{V}$ systems this is not true for the $\mathrm{D}$ systems. It is thus necessary to modify the calculation at this point. In the case of $\mathrm{D}$ systems $\mathscr{L}_{1}$ describes spontaneous emission due to the Einstein coefficients $A_{1}$ and $A_{2}$. Therefore $\mathscr{L}_{1} \rho_{\mathrm{ss}, i}$ consists of density matrix elements $\left\langle x_{k}|\rho| x_{l}\right\rangle$ where both states $\left|x_{k}\right\rangle$ and $\left|x_{l}\right\rangle$ lie in the same subspace $\mathscr{S}_{i}$. It is thus a superposition of eigenstates of $\mathscr{L}_{0}$ with zero as well as non-zero eigenvalues

$$
\mathscr{L}_{1} \rho_{\mathrm{ss}, i}=\sum_{j=0}^{2} \alpha_{i j} \rho_{\mathrm{ss}, j}+\tilde{\rho},
$$

where $\tilde{\rho}$ contains the contributions from the eigenstates for nonzero eigenvalues of $\mathscr{L}_{0}$. These eigenvalues are negative and of the order of $A_{3}$ and $\Omega_{3}$. The coefficients $\alpha_{i j}$ are calculated by means of the dual eigenstates $\rho_{\mathrm{ss}}^{i}[46]$

$$
\alpha_{i j}=\operatorname{Tr}\left(\rho_{\mathrm{ss}}^{j \dagger} \mathscr{L}_{1} \rho_{\mathrm{ss}, i}\right) .
$$

Inserting equation (3.51) into equation (3.49) one obtains

$$
\begin{aligned}
\rho\left(t_{0}+\Delta t ; \rho_{0, i}\right) & =\rho_{\mathrm{ss}, i}+\int_{0}^{\Delta t} \mathrm{~d} \tau\left(\sum_{j=0}^{2} \alpha_{i j} \rho_{\mathrm{ss}, j}+\mathrm{e}^{\mathscr{L}_{0} \tau} \tilde{\rho}\right) \\
& =\rho_{\mathrm{ss}, i}+\sum_{j=0}^{2} \alpha_{i j} \rho_{\mathrm{ss}, j} \Delta t+\left(\epsilon-\mathscr{L}_{0}\right)^{-1} \tilde{\rho} .
\end{aligned}
$$


For the $\tilde{\rho}$ term the upper integration limit can be extended to infinity since $\tilde{\rho}$ belongs to nonzero eigenvalues of $\mathscr{L}_{0}$ and is therefore rapidly damped. Now, $\mathscr{L}_{0}^{-1}$ is of the order of $A_{3}^{-1}$ and $\Omega_{3}^{-1}$ on $\tilde{\rho}$, and thus the last term in equation (3.53) is of the order of $\mathscr{L}_{1} /\left(A_{3}, \Omega_{3}\right)$ which is much smaller than $\alpha_{i j} \Delta t \sim \mathscr{L}_{1} \Delta t$, by equation (3.50). Therefore the last term in equation (3.53) can be neglected, and this equation then reveals that the $\alpha_{i j}$ 's have the meaning of transition rates between the subspaces $\mathcal{S}_{i}$ and $\mathcal{S}_{j}$

$$
p_{i j}=\alpha_{i j}
$$

As already mentioned above, the unperturbed Liouvillean $\mathscr{L}_{0}$ is identical to the one for two $\mathrm{V}$ systems. Therefore also the stationary states $\rho_{\mathrm{ss}, i}$ of the three subsystems are the same as those of the $\mathrm{V}$ systems given by equations (3.34a), (3.34b), and (3.34c). The last remaining problem in the calculation of the transition rates is the determination of the dual eigenstates $\rho_{\mathrm{ss}}^{i}$. Only those for eigenvalue 0 of $\mathscr{L}_{0}$ are needed. They are defined through

$$
\begin{gathered}
\operatorname{Tr}\left(\rho_{\mathrm{ss}}^{i \dagger} \rho_{\mathrm{ss}, j}\right)=\delta_{i j}, \quad i, j=0,1,2 \\
\operatorname{Tr}\left(\rho_{\mathrm{ss}}^{i \dagger} \mathscr{L}_{0} A\right)=0 \quad \text { for any matrix } A .
\end{gathered}
$$

The latter means $\mathscr{L}_{0}^{\dagger} \rho_{\mathrm{ss}}^{i}=0$, with the adjoint $\mathscr{L}_{0}^{\dagger}$ defined with respect to a scalar product given by $\operatorname{Tr}\left(A^{\dagger} B\right)$. The reciprocals $\rho_{\mathrm{ss}}^{i}$ are easily determined as follows. Since the Bloch equations conserve the trace one has

$$
0=\operatorname{Tr} \dot{\rho}=\operatorname{Tr} \mathscr{L}_{0} \rho
$$

for any $\rho$. Thus

$$
0=\operatorname{Tr}\left(\mathbb{1} \mathscr{L}_{0} \rho\right)=\operatorname{Tr}\left(\left(\mathscr{L}_{0}^{\dagger} \mathbb{1}\right) \rho\right)
$$

for any $\rho$ and therefore $\mathscr{L}_{0}^{\dagger} \mathbb{1}=0$. Now $\mathbb{1}$ can be written as a sum of terms purely from $\mathscr{S}_{0}, \mathscr{S}_{1}$, and $\mathscr{S}_{2}$ and, since the subspaces are invariant under $\mathscr{L}_{0}$, these terms must be annihilated by $\mathscr{L}_{0}^{\dagger}$ individually. This yields

$$
\begin{aligned}
& \rho_{\mathrm{ss}}^{0}=\left|e_{2}\right\rangle\left\langle e_{2}\right|, \\
& \rho_{\mathrm{ss}}^{1}=\left|s_{12}\right\rangle\left\langle s_{12}|+| a_{12}\right\rangle\left\langle a_{12}|+| s_{23}\right\rangle\left\langle s_{23}|+| a_{23}\right\rangle\left\langle a_{23}\right|, \\
& \rho_{\mathrm{ss}}^{2}=|g\rangle\left\langle g|+| s_{13}\right\rangle\left\langle s_{13}|+| a_{13}\right\rangle\left\langle a_{13}|+| e_{3}\right\rangle\left\langle e_{3}\right|,
\end{aligned}
$$

since the sum of the right hand sides indeed gives $\mathbb{1}$ and the normalization condition of equation (3.55) is fulfilled.

The transition rates are now obtained from equations (3.52), (3.54) and (3.34a)$(3.34 \mathrm{c}))$ as

$$
p_{01}=2 A_{1}, \quad p_{10}=\frac{A_{2} \Omega_{3}^{2}}{A_{3}^{2}+2 \Omega_{3}^{2}+4 \Delta_{3}^{2}}, \quad p_{12}=A_{1},
$$


and

$$
\begin{array}{r}
p_{21}=2 \frac{A_{2} \Omega_{3}^{2}\left(A_{3}^{2}+2 \Omega_{3}^{2}+4 \Delta_{3}^{2}\right)}{\left(A_{3}^{2}+2 \Omega_{3}^{2}+4 \Delta_{3}^{2}\right)^{2}+\left(A_{3}^{2}+4 \Delta_{3}^{2}\right)\left(\left|C_{3}\right|^{2}+2 A_{3} \operatorname{Re} C_{3}-4 \Delta_{3} \operatorname{Im} C_{3}\right)} \\
=\frac{2 A_{2} \Omega_{3}^{2}}{A_{3}^{2}+2 \Omega_{3}^{2}+4 \Delta_{3}^{2}}\left[1-2 \operatorname{Re} C_{3} \frac{A_{3}\left(A_{3}^{2}+4 \Delta_{3}^{2}\right)}{\left(A_{3}^{2}+2 \Omega_{3}^{2}+4 \Delta_{3}^{2}\right)^{2}}\right. \\
\left.+4 \operatorname{Im} C_{3} \frac{\Delta_{3}\left(A_{3}^{2}+4 \Delta_{3}^{2}\right)}{\left(A_{3}^{2}+2 \Omega_{3}^{2}+4 \Delta_{3}^{2}\right)^{2}}\right]+\mathcal{O}\left(C_{3}^{2}\right) .
\end{array}
$$

One sees that, for two D systems, only $p_{21}$ depends on the dipole coupling constants $C_{3}$ in first order. This contrasts with two $\mathrm{V}$ systems where both $p_{21}$ and $p_{12}$ depend on $C_{3}$. The physical reason for this is that transitions between bright periods for two D systems are due to decays and not due to the laser. Hence the transition rates should essentially be governed by the Einstein coefficients of these decays on the one hand and by the population of the states in the initial subsystem on the other hand. Therefore the parameter $C_{3}$ enters only through the quasi-stationary state $\rho_{\mathrm{ss}, i}$ of the initial subsystem. The absence of a linear $C_{1}$ and $C_{2}$ dependence can be understood from Figure 3.5(b) as follows. For most slow transitions between two subspaces there are two channels with rates $A_{j} \pm \operatorname{Re} C_{j}$ so that $\operatorname{Re} C_{j}$ cancels. States with a single decay channel lie in $\mathscr{S}_{1}$ and, by symmetry, they appear in pairs with different sign of $\operatorname{Re} C_{j}$.

As for the $\mathrm{V}$ systems one obtains the double jump rate $n_{\mathrm{DJ}}$ from the transition rates $p_{i j}$ by equation (3.45). Significant cooperative effects occur only as long as $\Omega_{3}$ and $\Delta_{3}$ are at least an order of magnitude smaller than $A_{3}$. When compared to non interacting systems, the cooperative effects are up to $30 \%$ for distances between one and two wave lengths and $5 \%$ around ten wave lengths, similar as for two $\mathrm{V}$ systems. For longer distances they are practically absent and this is consistent with the experimental results of Reference [23]. Figure 3.6 shows $p_{21}$ and $n_{\text {DJ versus the }}$ relative distance $r / \lambda_{3}$ for typical parameters, where $\lambda_{3}$ is the wave length of the strong transition.

The explicit results for arbitrary $r$ given here confirm the large-distance result of Reference [37] where it is argued that for $\lambda_{1}, \lambda_{3} \ll r \ll \lambda_{2}$ cooperative effects are "suppressed by the rapid decay on the fast transition". In fact, these effects are only to first order independent of the coupling parameter $C_{2}$; the second order contributions in $C_{2}$ are, however, negligible for the experimental values of reference [23]. The corresponding calculation can be found in Appendix A.

\subsubsection{Two dipole-interacting four-level systems as a model for two $\mathrm{Ba}^{+}$-ions}

The experiments of References [20, 19] used $\mathrm{Ba}^{+}$ions. As explained in Section 2.3 the relevant level scheme is modeled by the effective four-level configuration shown in Figure 2.3 (b). The $|1\rangle-|4\rangle$ transition is driven weakly and incoherently by a 

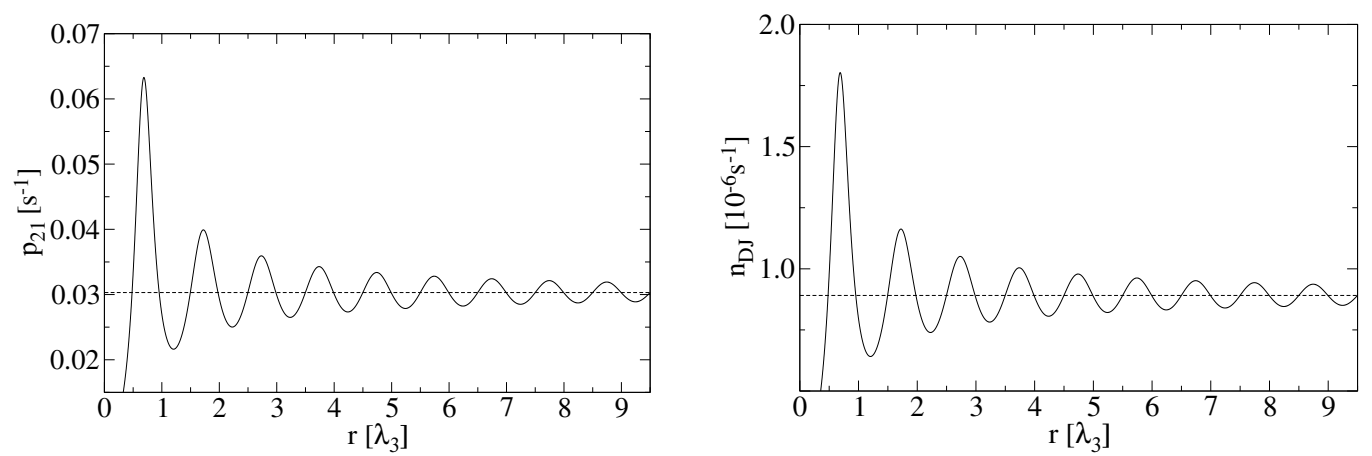

Figure 3.6: Transition rate $p_{21}$ and double jump rate $n_{D J}$ for two dipole-interacting $D$ systems. The dashed lines show the case of independent systems. Parameter values are $A_{1}=1 \mathrm{~s}^{-1}, A_{2}=1 \mathrm{~s}^{-1}, A_{3}=4 \times 10^{8} \mathrm{~s}^{-1}, \Omega_{3}=5 \times 10^{7} \mathrm{~s}^{-1}$, and $\Delta_{3}=0$.

lamp, while the $|1\rangle-|3\rangle$ transition is driven coherently by a strong laser. The Bloch equation can therefore be written as [47]

$$
\begin{aligned}
\dot{\rho} & =-\frac{\mathrm{i}}{\hbar}\left[H_{\text {cond }} \rho-\rho H_{\text {cond }}^{\dagger}\right]+\mathscr{R}_{W}(\rho)+\mathscr{R}(\rho) \\
& =\left\{\mathscr{L}_{0}\left(A_{2}, A_{3}, A_{4}, \Omega_{3}, \Delta_{3}, C_{2}, C_{3}, C_{4}\right)+\mathscr{L}_{1}\left(A_{1}, C_{1}, W\right)\right\} \rho
\end{aligned}
$$

where $\mathscr{R}_{W}(\rho)$ describes the incoherent driving as in Reference [47] and is given explicitly below. The Dicke states are defined in analogy to equation (3.23), and $H_{\text {cond }}$ and $\mathscr{R}(\rho)$ can be calculated as for the $\mathrm{V}$ and $\mathrm{D}$ systems and are given by

$$
\begin{gathered}
H_{\text {cond }}=\frac{\hbar}{2 \mathrm{i}}\left\{A_{1}\left[2\left|e_{2}\right\rangle\left\langle e_{2}|+| s_{12}\right\rangle\left\langle s_{12}|+| a_{12}\right\rangle\left\langle a_{12}\right|+\sum_{j=3}^{4}\left\{\left|s_{2 j}\right\rangle\left\langle s_{2 j}|+| a_{2 j}\right\rangle\left\langle a_{2 j}\right|\right\}\right]\right. \\
+\left(A_{2}+A_{4}\right)\left[2\left|e_{4}\right\rangle\left\langle e_{4}\right|+\sum_{j=1}^{3}\left\{\left|s_{j 4}\right\rangle\left\langle s_{j 4}|+| a_{j 4}\right\rangle\left\langle a_{j 4}\right|\right\}\right] \\
+\left(A_{3}-2 \mathrm{i} \Delta_{3}\right)\left[2\left|e_{3}\right\rangle\left\langle e_{3}\left|+\sum_{j=1}^{2}\left\{\left|s_{j 3}\right\rangle\left\langle s_{j 3}|+| a_{j 3}\right\rangle\left\langle a_{j 3}\right|\right\}+\right| s_{34}\right\rangle\left\langle s_{34}|+| a_{34}\right\rangle\left\langle a_{34}\right|\right] \\
+W\left[2|g\rangle\left\langle g|+2| e_{4}\right\rangle\left\langle e_{4}\right|+\sum_{j=1}^{3}\left\{\left|s_{j 4}\right\rangle\left\langle s_{j 4}|+| a_{j 4}\right\rangle\left\langle a_{j 4}\right|\right\}\right. \\
\left.\left.+\sum_{j=2}^{4}\left\{\left|s_{1 j}\right\rangle\left\langle s_{1 j}|+| a_{1 j}\right\rangle\left\langle a_{1 j}\right|\right\}\right]\right\} \\
+\frac{\hbar}{2 \mathrm{i}}\left\{C_{1}\left(\left|s_{12}\right\rangle\left\langle s_{12}|-| a_{12}\right\rangle\left\langle a_{12}\right|\right)+C_{2}\left(\left|s_{23}\right\rangle\left\langle s_{23}|-| a_{23}\right\rangle\left\langle a_{23}\right|\right)\right. \\
\left.+\sum_{j=3}^{4} C_{j}\left(\left|s_{1 j}\right\rangle\left\langle s_{1 j}|-| a_{1 j}\right\rangle\left\langle a_{1 j}\right|\right)\right\}
\end{gathered}
$$




$$
\begin{aligned}
+\frac{\hbar}{2} \Omega_{3}\left\{\sqrt{2}\left(|g\rangle\left\langle s_{13}|+| s_{13}\right\rangle\left\langle e_{3}\right|\right)\right. & +\left|s_{12}\right\rangle\left\langle s_{23}|-| a_{12}\right\rangle\left\langle a_{23}\right| \\
+ & \left.\left|s_{14}\right\rangle\left\langle s_{34}|+| a_{14}\right\rangle\left\langle a_{34}\right|+\text { h.c. }\right\}
\end{aligned}
$$

and

$$
\mathscr{R}(\rho)=\sum_{j=1}^{4}\left[\left(A_{j}+\operatorname{Re} C_{j}\right) R_{+}^{(j)} \rho R_{+}^{(j)^{\dagger}}+\left(A_{j}-\operatorname{Re} C_{j}\right) R_{-}^{(j)} \rho R_{-}^{(j)^{\dagger}}\right],
$$

with

$$
\begin{aligned}
& R_{+}^{(1)}=|g\rangle\left\langle s_{12}|+| s_{12}\right\rangle\left\langle e_{2}\right|+\frac{1}{\sqrt{2}}\left(\left|s_{13}\right\rangle\left\langle s_{23}|+| a_{13}\right\rangle\left\langle a_{23}|+| s_{14}\right\rangle\left\langle s_{24}|+| a_{14}\right\rangle\left\langle a_{24}\right|\right), \\
& R_{-}^{(1)}=|g\rangle\left\langle a_{12}|+| a_{12}\right\rangle\left\langle e_{2}\right|+\frac{1}{\sqrt{2}}\left(\left|a_{13}\right\rangle\left\langle s_{23}|-| s_{13}\right\rangle\left\langle a_{23}|+| a_{14}\right\rangle\left\langle s_{24}|-| s_{14}\right\rangle\left\langle a_{24}\right|\right), \\
& R_{+}^{(2)}=\left|e_{2}\right\rangle\left\langle s_{24}|+| s_{24}\right\rangle\left\langle e_{4}\right|+\frac{1}{\sqrt{2}}\left(\left|s_{12}\right\rangle\left\langle s_{14}|+| a_{12}\right\rangle\left\langle a_{14}|+| s_{23}\right\rangle\left\langle s_{34}|-| a_{23}\right\rangle\left\langle a_{34}\right|\right), \\
& R_{-}^{(2)}=\left|e_{2}\right\rangle\left\langle a_{24}|+| a_{24}\right\rangle\left\langle e_{4}\right|+\frac{1}{\sqrt{2}}\left(\left|s_{12}\right\rangle\left\langle a_{14}|-| a_{12}\right\rangle\left\langle s_{14}|+| s_{23}\right\rangle\left\langle a_{34}|+| a_{23}\right\rangle\left\langle s_{34}\right|\right), \\
& R_{+}^{(3)}=|g\rangle\left\langle s_{13}|+| s_{13}\right\rangle\left\langle e_{3}\right|+\frac{1}{\sqrt{2}}\left(\left|s_{12}\right\rangle\left\langle s_{23}|-| a_{12}\right\rangle\left\langle a_{23}|+| s_{14}\right\rangle\left\langle s_{34}|+| a_{14}\right\rangle\left\langle a_{34}\right|\right), \\
& R_{-}^{(3)}=|g\rangle\left\langle a_{13}|+| a_{13}\right\rangle\left\langle e_{3}\right|+\frac{1}{\sqrt{2}}\left(\left|s_{12}\right\rangle\left\langle a_{23}|+| a_{12}\right\rangle\left\langle s_{23}|+| s_{14}\right\rangle\left\langle a_{34}|-| a_{14}\right\rangle\left\langle s_{34}\right|\right), \\
& R_{+}^{(4)}=|g\rangle\left\langle s_{14}|+| s_{14}\right\rangle\left\langle e_{4}\right|+\frac{1}{\sqrt{2}}\left(\left|s_{12}\right\rangle\left\langle s_{24}|-| a_{12}\right\rangle\left\langle a_{24}|+| s_{13}\right\rangle\left\langle s_{34}|-| a_{13}\right\rangle\left\langle a_{34}\right|\right), \\
& R_{-}^{(4)}=|g\rangle\left\langle a_{14}|+| a_{14}\right\rangle\left\langle e_{4}\right|+\frac{1}{\sqrt{2}}\left(\left|s_{12}\right\rangle\left\langle a_{24}|+| a_{12}\right\rangle\left\langle s_{24}|+| s_{13}\right\rangle\left\langle a_{34}|+| a_{13}\right\rangle\left\langle s_{34}\right|\right) .
\end{aligned}
$$

The lamp term is obtained as in Reference [47] as

$$
\mathscr{R}_{W}(\rho)=W\left(R_{+}^{(4)} \rho R_{+}^{(4) \dagger}+R_{-}^{(4)} \rho R_{-}^{(4) \dagger}+R_{+}^{(4) \dagger} \rho R_{+}^{(4)}+R_{-}^{(4) \dagger} \rho R_{-}^{(4)}\right)
$$

where $W$ is the product of the spectral energy density of the lamp $W\left(\omega_{4}\right)$ and the Einstein $B$ coefficient of the $|1\rangle-|4\rangle$ transition

$$
W=\left(\boldsymbol{D}_{14} \cdot \boldsymbol{E}_{0}\right)^{2} \frac{W\left(\omega_{4}\right) \pi \mathrm{e}^{2}}{2 \epsilon_{0} \hbar^{2}} .
$$

Now the procedure is similar as for the D system. The Liouvillean $\mathscr{L}_{0}$ possesses three (quasi-) stationary states $\rho_{\mathrm{ss}, 0}, \rho_{\mathrm{ss}, 1}$, and $\rho_{\mathrm{ss}, 2}$ which coincide with those for the $\mathrm{V}$ systems in equations (3.34a)-(3.34c) and which are associated with the dark and the two bright periods. As before, one calculates $\rho\left(t_{0}+\Delta t\right)$ as in equation (3.49) and decomposes $\mathscr{L}_{1} \rho_{\mathrm{ss}, i}$ as in equation (3.51). Now, however, the reciprocals $\rho_{\mathrm{ss}}^{i}$ are 
more difficult to determine since $|4\rangle$ can decay into $|1\rangle$ as well as $|2\rangle$. An exact solution of $\mathscr{L}_{0}^{\dagger} \rho_{\mathrm{ss}}^{i}=0$ is rather elaborate. Therefore the decomposition

$$
\mathscr{L}_{0}^{\dagger}=\mathscr{L}_{0}^{\dagger(0)}\left(A_{2}, A_{3}, A_{4}, \Omega_{3}, \Delta_{3}, C_{3}\right)+\mathscr{L}_{0}^{\dagger(1)}\left(C_{2}, C_{4}\right)
$$

is applied and, by the computer algebra software Maple, $\rho_{\mathrm{ss}}^{i}$ is calculated to first order in perturbation theory with respect to $C_{2}$ and $C_{4}$, with the same constraint as in equation (3.55). In zeroth order $\left(C_{2}=C_{4}=0\right)$ the result is given by

$$
\begin{aligned}
\rho_{\mathrm{ss}}^{(0), 0}= & \left|e_{2}\right\rangle\left\langle e_{2}\left|+\frac{A_{2}}{A_{2}+A_{4}}\left[\left|s_{24}\right\rangle\left\langle s_{24}|+| a_{24}\right\rangle\left\langle a_{24}\right|\right]+\frac{A_{2}^{2}}{\left(A_{2}+A_{4}\right)^{2}}\right| e_{4}\right\rangle\left\langle e_{4}\right|, \\
\rho_{\mathrm{ss}}^{(0), 1}= & \left|s_{12}\right\rangle\left\langle s_{12}|+| a_{12}\right\rangle\left\langle a_{12}|+| s_{23}\right\rangle\left\langle s_{23}|+| a_{23}\right\rangle\left\langle a_{23}\right| \\
& +\frac{2 A_{2} A_{4}}{\left(A_{2}+A_{4}\right)^{2}}\left|e_{4}\right\rangle\left\langle e_{4}\right|+\frac{A_{4}}{A_{2}+A_{4}}\left[\left|s_{24}\right\rangle\left\langle s_{24}|+| a_{24}\right\rangle\left\langle a_{24}\right|\right] \\
& +\frac{A_{2}}{A_{2}+A_{4}}\left[\left|s_{14}\right\rangle\left\langle s_{14}|+| a_{14}\right\rangle\left\langle a_{14}|+| s_{34}\right\rangle\left\langle s_{34}|+| a_{34}\right\rangle\left\langle a_{34}\right|\right], \\
\rho_{\mathrm{ss}}^{(0), 2}= & |g\rangle\left\langle g|+| s_{13}\right\rangle\left\langle s_{13}|+| a_{13}\right\rangle\left\langle a_{13}|+| e_{3}\right\rangle\left\langle e_{3}\left|+\frac{A_{4}^{2}}{\left(A_{2}+A_{4}\right)^{2}}\right| e_{4}\right\rangle\left\langle e_{4}\right| \\
& +\frac{A_{4}}{A_{2}+A_{4}}\left[\left|s_{14}\right\rangle\left\langle s_{14}|+| a_{14}\right\rangle\left\langle a_{14}|+| s_{34}\right\rangle\left\langle s_{34}|+| a_{34}\right\rangle\left\langle a_{34}\right|\right],
\end{aligned}
$$

which is independent of $C_{3}$. The first order corrections are given by

$$
\begin{aligned}
\rho_{\mathrm{ss}}^{(1), 0}= & \frac{A_{4} \operatorname{Re} C_{2}}{A^{2}}\left[\left|s_{24}\right\rangle\left\langle s_{24}|-| a_{24}\right\rangle\left\langle a_{24}\right|\right], \\
\rho_{\mathrm{ss}}^{(1), 2}= & \frac{A_{2} \operatorname{Re} C_{4}}{A}\left[\frac{\Omega_{3}^{2}}{\left(A_{3}+2 A\right)\left[A\left(A+A_{3}\right)+\Omega_{3}^{2}+4 \Delta_{3}^{2}\right]}\left[\left|s_{34}\right\rangle\left\langle s_{34}|-| a_{34}\right\rangle\left\langle a_{34}\right|\right]\right. \\
& +\frac{\left(A+A_{3}\right)\left[\left(A_{3}+2 A\right)^{2}+4 \Delta_{3}^{2}\right]+\left(A_{3}+2 A\right) \Omega_{3}^{2}}{\left(A_{3}+2 A\right)^{2}\left[A\left(A+A_{3}\right)+\Omega_{3}^{2}+4 \Delta_{3}^{2}\right]}\left[\left|s_{14}\right\rangle\left\langle s_{14}|-| a_{14}\right\rangle\left\langle a_{14}\right|\right] \\
& -\mathrm{i} \frac{\Omega_{3}\left(A+A_{3}\right)\left(A_{3}+2 A+2 \mathrm{i} \Delta_{3}\right)}{\left(A_{3}+2 A\right)^{2}\left[A\left(A+A_{3}\right)+\Omega_{3}^{2}+4 \Delta_{3}^{2}\right]}\left[\left|s_{14}\right\rangle\left\langle s_{34}|-| a_{14}\right\rangle\left\langle a_{34}\right|\right. \\
& \left.\left.\quad+\left|s_{34}\right\rangle\left\langle s_{14}|-| a_{34}\right\rangle\left\langle a_{14}\right|\right]\right] \\
\rho_{\mathrm{ss}}^{(1), 1}= & -\rho_{\mathrm{ss}}^{(1), 0}-\rho_{\mathrm{ss}}^{(1), 2},
\end{aligned}
$$

with $A=A_{2}+A_{4}$.

As for the D systems the transition rates are given by

$$
p_{i j}=\operatorname{Tr}\left(\rho_{\mathrm{ss}}^{j \dagger} \mathscr{L}_{1} \rho_{\mathrm{ss}, i}\right)
$$

and one obtains for two dipole-interacting four-level systems of Figure 2.3 (b) to first order in $C_{2}$ and $C_{4}$

$$
p_{01}=2 A_{1} \quad p_{12}=A_{1} \quad p_{10}=\frac{A_{2} W}{A_{2}+A_{4}} \frac{A_{3}^{2}+\Omega_{3}^{2}+4 \Delta_{3}^{2}}{A_{3}^{2}+2 \Omega_{3}^{2}+4 \Delta_{3}^{2}}
$$



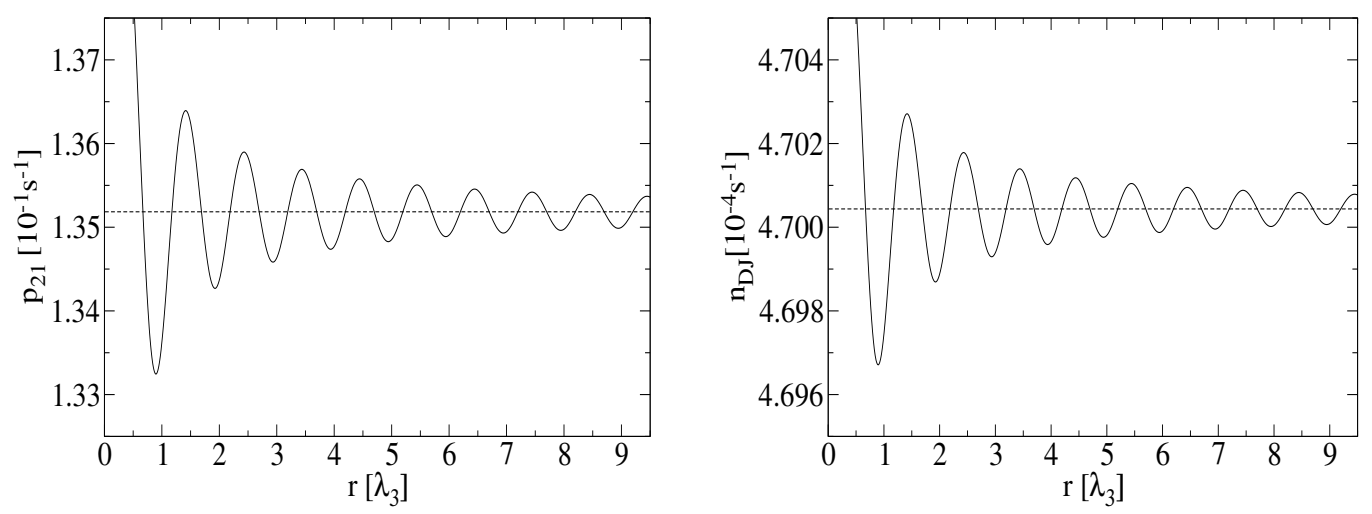

Figure 3.7: Transition rate $p_{21}$ and double-jump rate $n_{D J}$ for two dipole-interacting four-level systems, with optimal $\Omega_{3}=\frac{1}{2} \sqrt{\sqrt{5}-1} \sqrt{A_{3}^{2}+4 \Delta_{3}^{2}}$ and all other parameters as in the experiment [19]. The dashed lines show the case of independent systems.

and

$$
\begin{gathered}
p_{21}=2 A_{2} W \frac{\left(A_{3}^{2}+\Omega_{3}^{2}+4 \Delta_{3}^{2}\right)\left(A_{3}^{2}+2 \Omega_{3}^{2}+4 \Delta_{3}^{2}\right)+\left(A_{3}^{2}+4 \Delta_{3}^{2}\right) B}{\left(A_{2}+A_{4}\right)\left(A_{3}^{2}+2 \Omega_{3}^{2}+4 \Delta_{3}^{2}\right)^{2}+\left(A_{3}^{2}+4 \Delta_{3}^{2}\right) B} \\
=\frac{2 A_{2} W}{A_{2}+A_{4}}\left[\frac{A_{3}^{2}+\Omega_{3}^{2}+4 \Delta_{3}^{2}}{A_{3}^{2}+2 \Omega_{3}^{2}+4 \Delta_{3}^{2}}+2 \operatorname{Re} C_{3} \frac{A_{3}^{3} \Omega_{3}^{2}}{\left[A_{3}^{2}+2 \Omega_{3}^{2}+4 \Delta_{3}^{2}\right]^{3}}\right. \\
\left.\quad-4 \operatorname{Im} C_{3} \frac{\Delta_{3} A_{3}^{2} \Omega_{3}^{2}}{\left[A_{3}^{2}+2 \Omega_{3}^{2}+4 \Delta_{3}^{2}\right]^{3}}\right]+\mathcal{O}\left(C_{3}^{2}\right),
\end{gathered}
$$

with $B=\left|C_{3}\right|^{2}+2 A_{3} \operatorname{Re} C_{3}-4 \Delta_{3} \operatorname{Im} C_{3}$. It is seen that $p_{01}, p_{10}$, and $p_{12}$ are independent of the coupling parameters and are thus the same as for non-interacting systems.

These results for two four-level systems show great similarity with those for the two D systems of the preceding section. In both cases only $p_{21}$ depends to first order on $C_{3}$, the coupling parameter associated with the laser-driven transition. However, cooperative effects are significantly smaller for the two four-level systems. For fixed laser detuning, the effect of $C_{3}$ becomes maximal for $\Omega_{3}=\frac{1}{2} \sqrt{\sqrt{5}-1} \sqrt{A_{3}+\Delta_{3}^{2}}$. For this value of $\Omega_{3}$, Figure 3.7 shows the transition rate $p_{21}$ from a double intensity period to a unit-intensity period and the double jump rate $n_{\mathrm{DJ}}$ over the relative distance $r / \lambda_{3}$, with the other parameters as in the experiment $[19,20]$. Despite the optimal choice of the Rabi frequency, $\Omega_{3}$, the deviations from the value for noninteracting systems are very small. Already for a distance of about a wave length $\lambda_{3}$, they are not more than $1 \%$ for $p_{21}$ when compared to non-interacting systems, while for $n_{\mathrm{DJ}}$ they are less than $1 \%$. 


\subsection{Three dipole-interacting atoms}

Although the results of the previous section seem to contradict the findings of References $[19,20]$ a direct quantitative comparison with the experiments is not possible since explicit experimental data was only provided for three $\mathrm{Ba}^{+}$ions.

The aim of this section is to narrow this gap by investigating three dipoleinteracting three-level systems in V configuration and in D configuration (see Figures 2.1 and 2.2), respectively, and to compare the results with those for two such systems. For three systems this becomes much more complicated since one has to deal with $729 \times 729$ density matrices, and in order to do this we use group theoretical methods to exploit the symmetry of the problem.

A full description of the $\mathrm{Ba}^{+}$experiment $[19,20]$ requires the treatment of three of the four-level systems of Reference [46]. This is rather complex with the methods presented so far, due to the larger number levels involved. This section is therefore completed by the presentation of a simplified model which reduces the computational effort and allows the analytical treatment of three four-level systems.

\subsubsection{Conditional Hamiltonian and reset state}

In the following we investigate three dipole-interacting three-level systems both in a V-type and in a D-type configuration as shown in Figures 2.1 and 2.2. As before the conditions

$$
\Omega_{3}, A_{3} \gg \Omega_{2}
$$

for the $\mathrm{V}$ systems and

$$
\Omega_{3}, A_{3} \gg A_{1}, A_{2}
$$

for the D systems should hold in order to have macroscopic bright and dark periods. From the general result (3.13) in section 3.1 one can read off the conditional Hamiltonian for three dipole-interacting $\mathrm{V}$ systems as

$$
\begin{aligned}
H_{\text {cond }}= & \sum_{i=1}^{3} \frac{\hbar}{2 \mathrm{i}}\left(A_{3}-2 \mathrm{i} \Delta_{3}\right) S_{i 3}^{+} S_{i 3}^{-}+\sum_{i=1}^{3} \sum_{j=2}^{3} \frac{\hbar}{2}\left[\Omega_{j} S_{i j}^{-}+\text {h.c. }\right] \\
& +\sum_{\substack{k, l=1 \\
k<l}}^{3} \frac{\hbar}{2 \mathrm{i}} C_{k l}^{(3)}\left(S_{k 3}^{+} S_{l 3}^{-}+S_{l 3}^{+} S_{k 3}^{-}\right)
\end{aligned}
$$

and for three dipole-interacting D systems as

$$
\begin{aligned}
H_{\text {cond }}= & \sum_{i=1}^{3} \sum_{j=1}^{3} \frac{\hbar}{2 \mathrm{i}} A_{j} S_{i j}^{+} S_{i j}^{-}+\sum_{i=1}^{3} \frac{\hbar}{2}\left[\left[\Omega_{3} S_{i 3}^{-}+\text {h.c. }\right]-2 \Delta_{3} S_{i 3}^{+} S_{i 3}^{-}\right] \\
& +\sum_{\substack{k, l=1 \\
k<l}}^{3} \sum_{j=1}^{3} \frac{\hbar}{2 \mathrm{i}} C_{k l}^{(j)}\left(S_{k j}^{+} S_{l j}^{-}+S_{l j}^{+} S_{k j}^{-}\right)
\end{aligned}
$$


with $S_{i j}^{ \pm}$defined as in the treatment of two atoms. The reset state is given by

$$
\mathscr{R}(\rho)=\sum_{i=1}^{3} \sum_{j=1}^{3} A_{j} S_{i j}^{-} \rho S_{i j}^{+}+\sum_{\substack{k, l=1 \\ k<l}}^{3} \sum_{j=1}^{3} \operatorname{Re} C_{k l}^{(j)}\left(S_{k j}^{-} \rho S_{l j}^{+}+S_{l j}^{-} \rho S_{k j}^{+}\right),
$$

where for the $\mathrm{V}$ systems only the terms with $A_{3}$ and $\operatorname{Re} C_{k l}^{(3)}$ have to be taken into account. In contrast to the case of two atoms there are now three dipole-dipole interaction parameters for each transition

$$
C_{k l}^{(j)}=\frac{3 A_{j}}{2} \mathrm{e}^{\mathrm{i} a_{k l}^{(j)}}\left[\frac{1}{\mathrm{i} a_{k l}^{(j)}}\left(1-\cos ^{2} \theta_{k l}\right)+\left(\frac{1}{a_{k l}^{(j) 2}}-\frac{1}{\mathrm{i} a_{k l}^{(j) 3}}\right)\left(1-3 \cos ^{2} \theta_{k l}\right)\right],
$$

one for each pair of atoms. They depend on the inter-atomic distances and the angles between the dipole moments and the line connecting each pair of atoms. In general all these quantities can assume different values. They are basically determined by the geometrical arrangement of the atoms in the trap, the direction of the applied magnetic field used to align the dipole-moments of the atoms, and the direction of incidence of the lasers. Depending on the kind of ion trap used in an experiment there are two possible geometric configurations. In the usual ion traps utilized in the experiments of references $[19,20]$ and [23] the atoms form an equilateral triangle. Since the inter-atomic distance is, in this case, the same for all pairs of atoms, $r=r_{k l}$, one has $a_{k l}^{(j)}=a_{j}=2 \pi r / \lambda_{j}$, so that the coupling parameters only depend on the atom number via the angles $\vartheta_{k l}$. In the linear ion traps used in the newer experiments $[22,24,25,27]$, however, the atoms are positioned on a line giving $r_{12}=r_{23}=r_{13} / 2$. In following only the first case will be discussed.

For simplicity it would be preferable to have identical coupling parameters for each pair of atoms (i.e. $C_{k l}^{(j)} \equiv C_{j}$ ). This would be the case if the angles between the dipole moments and the line connecting two atoms were the same for all pairs of atoms. The dipole moments are usually aligned in the experiments by applying a homogeneous magnetic field. In addition, it has already been assumed implicitly that the Rabi frequencies for a given transition have the same phase $\mathrm{e}^{\mathrm{i} \boldsymbol{k}_{\mathrm{L}} \cdot \boldsymbol{r}_{i}}$ for each atom. The latter is achieved most easily by setting the laser direction perpendicular to the plane of the triangle, which gives $\boldsymbol{k}_{\mathrm{L}} \cdot \boldsymbol{r}_{i}=0$. With the dipole moments perpendicular to the laser direction, as in the experiment of Reference [19], it is in this case impossible to obtain the same value of $\cos \vartheta_{k l}$ for the three angles $\vartheta_{k l}$ as illustrated in Figure 3.8. If on the other hand, by choosing a laser direction in the plane of the triangle, one fixes the $\vartheta_{k l}=\pi / 2$ which gives maximal values for $C_{k l}^{(j)}$, one has to adjust the inter-atomic distance $r$ to the right value in order to have $\mathrm{e}^{\mathrm{i} \boldsymbol{k}_{\mathrm{L}} \cdot \boldsymbol{r}_{i}}=1$ for all atoms. This is difficult to achieve experimentally.

However, in spite of this, it will be assumed $C_{k l}^{(j)} \equiv C_{j}$ and $\boldsymbol{k}_{\mathrm{L}} \cdot \boldsymbol{r}_{i}=0$ in the following because this case leads to maximal cooperative effects and can be seen as a limiting case for all other possible configurations. 


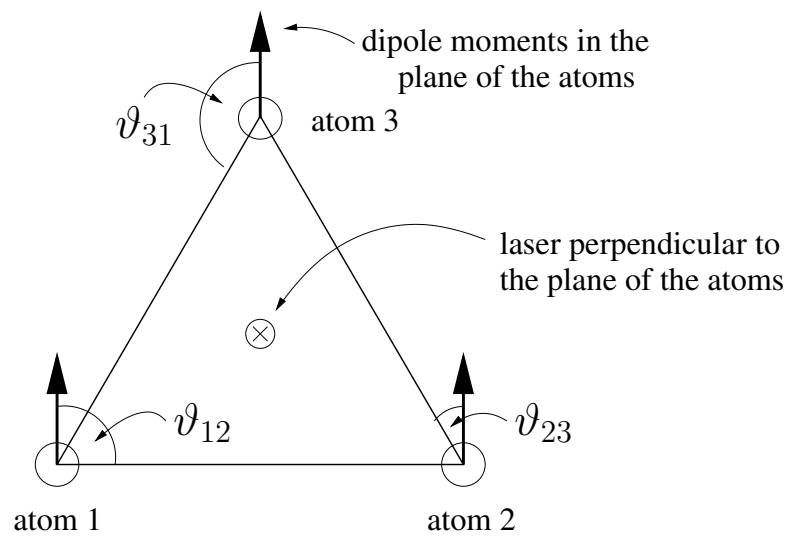

Figure 3.8: Geometry of the atoms in the trap. The arrows symbolize the dipole moments. In the picture the angles have the values $\vartheta_{12}=\pi / 2, \vartheta_{23}=\pi / 6$, and $\vartheta_{31}=5 \pi / 6$ leading to $\cos ^{2} \vartheta_{12}=0$, and $\cos ^{2} \vartheta_{23}=\cos ^{2} \vartheta_{31}=3 / 4$.

\subsubsection{Group theoretical methods}

As in the case of two atoms it is necessary for the calculation of the transition rates for both $\mathrm{V}$ systems and $\mathrm{D}$ systems to compute the quasi-steady states $\rho_{\mathrm{ss}, i}$, i.e. to solve the linear equation

$$
\mathscr{L}_{0} \rho_{\mathrm{ss}, i}=0 .
$$

In addition, for $\mathrm{V}$ systems the first order term

$$
\rho_{i}^{(1)}=\left(\epsilon-\mathscr{L}_{0}\right)^{-1} \mathscr{L}_{1} \rho_{\mathrm{ss}, i}
$$

of equation (3.31) has to be calculated, which was done by solving

$$
\mathscr{L}_{0} \rho_{i}^{(1)}=\mathscr{L}_{1} \rho_{\mathrm{ss}, i}
$$

Equations (3.74) and (3.75) are linear equations for the 729 matrix elements of $\rho_{\mathrm{ss}, i}$ and $\rho_{i}^{(1)}$, respectively. It would be therefore advantageous to restrict the problem to smaller subspaces. This is indeed possible with the use of group theoretical methods which exploit the symmetries of the system.

In the case of two atoms it was convenient to use the Dicke basis, i.e., the basis consisting of the symmetric and antisymmetric linear combinations of the product states. Generally speaking, this means using a basis which is adapted with respect to the symmetry group $\mathcal{S}_{2}$ of permutations of two atoms. The symmetric and antisymmetric states correspond to the irreducible representations of this group. Hence, for three three-level systems, a basis that is adapted to the symmetry group $\mathcal{S}_{3}$ of permutations of three particles is used. On the subspace spanned by the product states with all three atoms in different states the irreducible representations of the $\mathcal{S}_{3}$ are the two one-dimensional representations mentioned above and another 
two equivalent two-dimensional representations. This leads to the states

$$
\begin{aligned}
& \left|s_{123}\right\rangle=\frac{1}{\sqrt{6}}(|1\rangle|2\rangle|3\rangle+|2\rangle|3\rangle|1\rangle+|3\rangle|1\rangle|2\rangle+|1\rangle|3\rangle|2\rangle+|2\rangle|1\rangle|3\rangle+|3\rangle|2\rangle|1\rangle), \\
& \left|a_{123}\right\rangle=\frac{1}{\sqrt{6}}(|1\rangle|2\rangle|3\rangle+|2\rangle|3\rangle|1\rangle+|3\rangle|1\rangle|2\rangle-|1\rangle|3\rangle|2\rangle-|2\rangle|1\rangle|3\rangle-|3\rangle|2\rangle|1\rangle), \\
& \left|b_{123}\right\rangle=\frac{1}{\sqrt{12}}(2|1\rangle|2\rangle|3\rangle-|2\rangle|3\rangle|1\rangle-|3\rangle|1\rangle|2\rangle+2|1\rangle|3\rangle|2\rangle-|2\rangle|1\rangle|3\rangle-|3\rangle|2\rangle|1\rangle), \\
& \left|c_{123}\right\rangle=\frac{1}{2}(|2\rangle|3\rangle|1\rangle-|3\rangle|1\rangle|2\rangle-|2\rangle|1\rangle|3\rangle+|3\rangle|2\rangle|1\rangle), \\
& \left|d_{123}\right\rangle=\frac{1}{\sqrt{12}}(2|1\rangle|2\rangle|3\rangle-|2\rangle|3\rangle|1\rangle-|3\rangle|1\rangle|2\rangle-2|1\rangle|3\rangle|2\rangle+|2\rangle|1\rangle|3\rangle+|3\rangle|2\rangle|1\rangle), \\
& \left|e_{123}\right\rangle=\frac{1}{2}(|2\rangle|3\rangle|1\rangle-|3\rangle|1\rangle|2\rangle+|2\rangle|1\rangle|3\rangle-|3\rangle|2\rangle|1\rangle)
\end{aligned}
$$

in the case where all three atoms are in different states. For the remaining states one then easily gets for $i, j=1,2,3, i \neq j$,

$$
\begin{aligned}
\left|s_{i j j}\right\rangle & =\frac{1}{\sqrt{3}}(|i\rangle|j\rangle|j\rangle+|j\rangle|j\rangle|i\rangle+|j\rangle|i\rangle|j\rangle), \\
\left|b_{i j j}\right\rangle & =\frac{1}{\sqrt{6}}(2|i\rangle|j\rangle|j\rangle-|j\rangle|j\rangle|i\rangle-|j\rangle|i\rangle|j\rangle), \\
\left|c_{i j j}\right\rangle & =\frac{1}{\sqrt{2}}(|j\rangle|j\rangle|i\rangle-|j\rangle|i\rangle|j\rangle)
\end{aligned}
$$

if two atoms are in the same state and

$$
|g\rangle=|1\rangle|1\rangle|1\rangle, \quad\left|e_{2}\right\rangle=|2\rangle|2\rangle|2\rangle, \quad\left|e_{3}\right\rangle=|3\rangle|3\rangle|3\rangle
$$

if all three atoms are in the same state. Similarly to the situation with two atoms, the reset state can then be written as a sum of three density matrices of pure states

$$
\mathscr{R}(\rho)=\sum_{j=1}^{3}\left\{\left(A_{j}+2 \operatorname{Re} C_{j}\right) R_{1}^{(j)} \rho R_{1}^{(j)^{\dagger}}+\left(A_{j}-\operatorname{Re} C_{j}\right)\left[R_{2}^{(j)} \rho R_{2}^{(j)^{\dagger}}+R_{3}^{(j)} \rho R_{3}^{(j)^{\dagger}}\right]\right\}
$$

with

$$
\begin{aligned}
& R_{1}^{(j)}=\frac{1}{\sqrt{3}}\left(S_{1 j}^{-}+S_{2 j}^{-}+S_{3 j}^{-}\right), \\
& R_{2}^{(j)}=\frac{1}{\sqrt{6}}\left(2 S_{1 j}^{-}-S_{2 j}^{-}-S_{3 j}^{-}\right),
\end{aligned}
$$




$$
R_{3}^{(j)}=\frac{1}{\sqrt{2}}\left(S_{2 j}^{-}-S_{3 j}^{-}\right)
$$

For the solution of equations (3.74) and (3.75) there are two properties of $\mathscr{L}_{0}$ which allow the restriction of the equations to smaller subspaces. Analogous to two atoms there are four subspaces

$$
\begin{aligned}
& \mathscr{S}_{0}=\left\{\left|e_{2}\right\rangle\right\}, \\
& \mathscr{S}_{1}=\left\{\left|s_{122}\right\rangle,\left|b_{122}\right\rangle,\left|c_{122}\right\rangle,\left|s_{322}\right\rangle,\left|b_{322}\right\rangle,\left|c_{322}\right\rangle\right\}, \\
& \mathscr{S}_{2}=\left\{\left|s_{211}\right\rangle,\left|b_{211}\right\rangle,\left|c_{211}\right\rangle,\left|s_{123}\right\rangle,\left|a_{123}\right\rangle,\left|b_{123}\right\rangle,\left|c_{123}\right\rangle,\right. \\
&\left.\left|d_{123}\right\rangle,\left|e_{123}\right\rangle,\left|s_{233}\right\rangle,\left|b_{233}\right\rangle,\left|c_{233}\right\rangle\right\}, \\
&\left.\mathscr{S}_{3}=\left\{|g\rangle,\left|s_{311}\right\rangle,\left|b_{311}\right\rangle,\left|c_{311}\right\rangle,\left|s_{133}\right\rangle,\left|b_{133}\right\rangle,\left|c_{133},\right| e_{3}\right\rangle\right\}
\end{aligned}
$$

which are only coupled by the weak parameters, $\Omega_{2}$ for the $\mathrm{V}$ systems and $A_{1}, A_{2}$ for the $\mathrm{D}$ systems. Consequently, the space of density matrix elements can be divided into 16 subspaces $\mathscr{S}_{i j}$ with the same property. Let $\left|x_{i}\right\rangle, i=1 \ldots 27$ be a numbering of the states (3.76). Then these subspaces are given by

$$
\left.\mathscr{S}_{i j}=\left\{\left\langle x_{k}|\rho| x_{l}\right\rangle|| x_{k}\right\rangle \in \mathscr{S}_{i},\left|x_{l}\right\rangle \in \mathscr{S}_{j}\right\} \text {. }
$$

Since $\mathscr{L}_{0}$ is independent of $\Omega_{2}$ and $A_{1}, A_{2}$ respectively they are invariant with respect to $\mathscr{L}_{0}$.

In addition the conditional Hamiltonian $H_{\text {cond }}$ and the reset state $\mathscr{R}(\rho)$ and therefore also $\mathscr{L}_{0}$ are invariant under the exchange of atoms, as can be seen from equations (3.71), (3.72), and (3.73). Hence, subspaces which consist of all density matrix elements which belong to a particular irreducible representation of $\mathcal{S}_{3}$ are also invariant with respect to $\mathscr{L}_{0}$. Since the density matrix elements form a representation of $\mathcal{S}_{3}$ which is a tensor product of twice the representation spanned by the Dicke basis of equation (3.76) the new irreducible representations are easily found. The density matrix elements

$$
\begin{array}{cc}
\left|s_{\alpha}\right\rangle\left\langle s_{\beta}|, \quad| a_{\alpha}\right\rangle\left\langle a_{\beta}\right|, & \\
\frac{1}{2}\left(\left|b_{\alpha}\right\rangle\left\langle b_{\beta}|+| c_{\alpha}\right\rangle\left\langle c_{\beta}\right|\right), & \frac{1}{2}\left(\left|d_{\alpha}\right\rangle\left\langle d_{\beta}|+| e_{\alpha}\right\rangle\left\langle e_{\beta}\right|\right), \\
\frac{1}{2}\left(\left|b_{\alpha}\right\rangle\left\langle e_{\beta}|-| c_{\alpha}\right\rangle\left\langle d_{\beta}\right|\right), & \frac{1}{2}\left(\left|e_{\alpha}\right\rangle\left\langle b_{\beta}|-| d_{\alpha}\right\rangle\left\langle c_{\beta}\right|\right)
\end{array}
$$

belong to the symmetric representation, the elements

$$
\begin{array}{cc}
\left|s_{\alpha}\right\rangle\left\langle a_{\beta}|, \quad| a_{\alpha}\right\rangle\left\langle s_{\beta}\right|, & \\
\frac{1}{2}\left(\left|b_{\alpha}\right\rangle\left\langle c_{\beta}|-| c_{\alpha}\right\rangle\left\langle b_{\beta}\right|\right), & \frac{1}{2}\left(\left|d_{\alpha}\right\rangle\left\langle e_{\beta}|-| e_{\alpha}\right\rangle\left\langle d_{\beta}\right|\right), \\
\frac{1}{2}\left(\left|b_{\alpha}\right\rangle\left\langle d_{\beta}|+| c_{\alpha}\right\rangle\left\langle e_{\beta}\right|\right), & \frac{1}{2}\left(\left|d_{\alpha}\right\rangle\left\langle b_{\beta}|+| e_{\alpha}\right\rangle\left\langle c_{\beta}\right|\right)
\end{array}
$$


belong to the antisymmetric representation, and the remaining 24 possible linear combinations form two-dimensional representations. Here $\alpha$ and $\beta$ stand for one of the subscripts of the Dicke states. By transforming the Liouvillean $\mathscr{L}_{0}$ into this new basis each of the 16 invariant subspaces $\mathscr{S}_{i j}$ is in itself decomposed into four invariant subspaces connected to the elements belonging to the symmetric, antisymmetric, and the two dimensional representations, respectively. For the calculation of both the quasi-steady states $\rho_{\mathrm{ss}, i}$ and the transition rates for the $\mathrm{V}$ systems only the symmetric subspaces are needed. With these two simplifications the dimension of the system of linear equations needed for the calculation reduces considerably (namely to a maximum of 20 for the calculation of $p_{23}$ and $p_{32}$ ).

\subsubsection{Transition rates}

The transition rates can then be calculated as explained in section 3.2. For three $\mathrm{V}$ systems the equivalent of equation (3.32) is given by

$$
\begin{aligned}
\frac{\mathrm{d}}{\mathrm{d} t}\left\langle e_{2}|\rho| e_{2}\right\rangle= & \sqrt{3} \Omega_{2} \operatorname{Im}\left\langle s_{122}|\rho| e_{2}\right\rangle, \\
\frac{\mathrm{d}}{\mathrm{d} t} \sum_{x_{i} \in \mathscr{S}_{1}}\left\langle x_{i}|\rho| x_{i}\right\rangle= & \Omega_{3} \operatorname{Im}\left[2\left\langle s_{112}|\rho| s_{122}\right\rangle-\left\langle b_{112}|\rho| b_{122}\right\rangle-\left\langle c_{112}|\rho| c_{122}\right\rangle\right. \\
- & \sqrt{3}\left\langle s_{122}|\rho| e_{2}\right\rangle+\sqrt{2}\left\langle s_{123}|\rho| s_{223}\right\rangle-\frac{1}{\sqrt{2}}\left(\left\langle b_{123}|\rho| b_{223}\right\rangle+\left\langle c_{123}|\rho| c_{223}\right\rangle\right) \\
& \left.+\sqrt{\frac{3}{2}}\left(\left\langle d_{123}|\rho| c_{223}\right\rangle-\left\langle e_{123}|\rho| b_{223}\right\rangle\right)\right]-\frac{\mathrm{d}}{\mathrm{d} t}\left\langle e_{2}|\rho| e_{2}\right\rangle, \\
- & \sqrt{3}\left\langle s_{122}|\rho| e_{2}\right\rangle+\sqrt{2}\left\langle s_{123}|\rho| s_{223}\right\rangle-\frac{1}{\sqrt{2}}\left(\left\langle b_{123}|\rho| b_{223}\right\rangle+\left\langle c_{123}|\rho| c_{223}\right\rangle\right) \\
\frac{\mathrm{d} t}{\mathrm{~d} t} \sum_{x_{i} \in \mathscr{S}_{2}}\left\langle x_{i}|\rho| x_{i}\right\rangle= & -\Omega_{3} \operatorname{Im}\left[2\left\langle s_{112}|\rho| s_{122}\right\rangle-\left\langle b_{112}|\rho| b_{122}\right\rangle-\left\langle c_{112}|\rho| c_{122}\right\rangle\right. \\
& \left.+\sqrt{\frac{3}{2}}\left(\left\langle d_{123}|\rho| c_{223}\right\rangle-\left\langle e_{123}|\rho| b_{223}\right\rangle\right)\right]-\frac{\mathrm{d}}{\mathrm{d} t} \sum_{x_{i} \in \mathscr{S}_{3}}\left\langle x_{i}|\rho| x_{i}\right\rangle \\
\frac{\mathrm{d}}{\mathrm{d} t} \sum_{x_{i} \in \mathcal{S}_{3}}\left\langle x_{i}|\rho| x_{i}\right\rangle= & \Omega_{2} \operatorname{Im}\left[\frac{1}{\sqrt{2}}\left(\left\langle b_{113}|\rho| b_{123}\right\rangle+\left\langle c_{311}|\rho| c_{123}\right\rangle\right)\right. \\
& +\sqrt{\frac{3}{2}}\left(\left\langle b_{311}|\rho| e_{123}\right\rangle-\left\langle c_{311}|\rho| d_{123}\right\rangle\right)-\sqrt{3}\left\langle g|\rho| s_{211}\right\rangle \\
& \left.-\sqrt{2}\left\langle s_{311}|\rho| s_{233}\right\rangle-\left(\left\langle s_{133}|\rho| s_{233}\right\rangle+\left\langle b_{133}|\rho| b_{233}\right\rangle+\left\langle c_{133}|\rho| c_{233}\right\rangle\right)\right] .
\end{aligned}
$$

The resulting transition rates are

$$
p_{01}=3 \frac{A_{3} \Omega_{2}^{2}}{\Omega_{3}^{2}},
$$




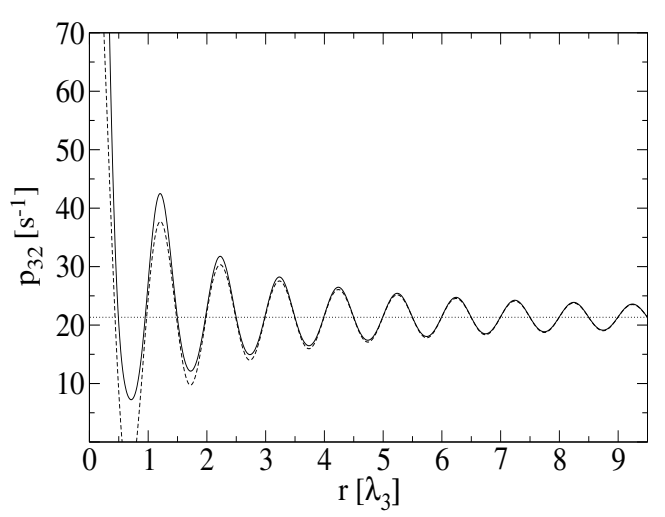

Figure 3.9: Transition rate $p_{32}$ for three dipole-interacting $V$ systems plotted versus the inter-atomic distance $r$ in units of the wavelength $\lambda_{3}$ of the strong transition. Solid line: $p_{32}$ up to second order in $C_{3}$. Dashed line: first order. Dotted line: independent systems. Parameter values are $A_{3}=2 \times 10^{8} \mathrm{~s}^{-1}$, $\Omega_{3}=5 \times 10^{7} \mathrm{~s}^{-1}$, and $\Omega_{2}=10^{4} \mathrm{~s}^{-1}$.

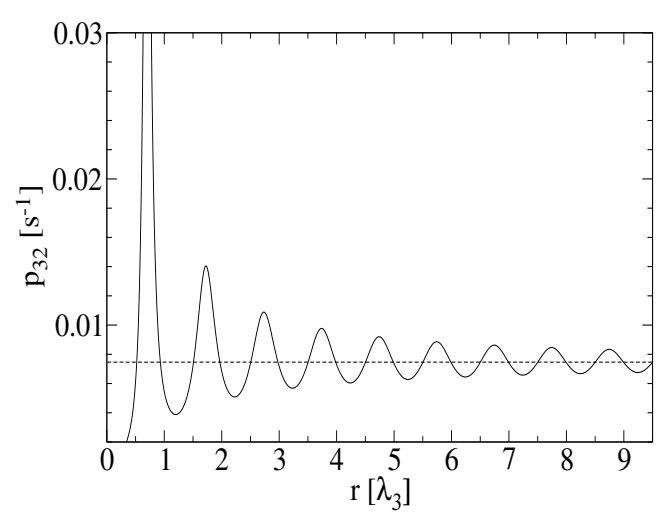

Figure 3.10: Transition rate $p_{32}$ for two dipole-interacting $D$ systems. Dashed line: independent systems. Parameter values are $A_{1}=1 \mathrm{~s}^{-1}, A_{2}=1 \mathrm{~s}^{-1}$, $A_{3}=2 \times 10^{8} \mathrm{~s}^{-1}$, and $\Omega_{3}=10^{7} \mathrm{~s}^{-1}$.

$$
\begin{aligned}
& p_{10}=\frac{A_{3} \Omega_{2}^{2}\left(A_{3}^{2}+4 \Delta_{3}^{2}\right)}{\Omega_{3}^{2}\left(A_{3}^{2}+2 \Omega_{3}^{2}+4 \Delta_{3}^{2}\right)}, \\
& p_{12}=2 \frac{A_{3} \Omega_{2}^{2}}{\Omega_{3}^{2}}\left[1+2 \operatorname{Re} C_{3} \frac{A_{3}}{A_{3}^{2}+2 \Omega_{3}^{2}+4 \Delta_{3}^{2}}-4 \operatorname{Im} C_{3} \frac{\Delta_{3}}{A_{3}^{2}+2 \Omega_{3}^{2}+4 \Delta_{3}^{2}}\right] \\
& p_{21}=2 \frac{A_{3} \Omega_{2}^{2}\left(A_{3}^{2}+4 \Delta_{3}^{2}\right)}{\Omega_{3}^{2}\left(A_{3}^{2}+2 \Omega_{3}^{2}+4 \Delta_{3}^{2}\right)}\left[1+2 \operatorname{Re} C_{3} \frac{A_{3}\left(A_{3}^{2}+4 \Omega_{3}^{2}+4 \Delta_{3}^{2}\right)}{\left(A_{3}^{2}+2 \Omega_{3}^{2}+4 \Delta_{3}^{2}\right)^{2}}\right. \\
& \left.\quad-4 \operatorname{Im} C_{3} \frac{\Delta_{3}\left(A_{3}^{2}+4 \Omega_{3}^{2}+4 \Delta_{3}^{2}\right)}{\left(A_{3}^{2}+2 \Omega_{3}^{2}+4 \Delta_{3}^{2}\right)^{2}}\right] \\
& p_{23}=\frac{A_{3} \Omega_{2}^{2}}{\Omega_{3}^{2}}\left[1+4 \operatorname{Re} C_{3} \frac{\Delta_{3}}{A_{3}^{2}+2 \Omega_{3}^{2}+4 \Delta_{3}^{2}}-4 \operatorname{Im} C_{3} \frac{\Delta_{3}^{2}+2 \Omega_{3}^{2}+4 \Delta_{3}^{2}}{A_{3}^{2}}\right] \\
& p_{32}=3 \frac{A_{3} \Omega_{2}^{2}\left(A_{3}^{2}+4 \Delta_{3}^{2}\right)}{\Omega_{3}^{2}\left(A_{3}^{2}+2 \Omega_{3}^{2}+4 \Delta_{3}^{2}\right)}\left[1+4 \operatorname{Re} C_{3} \frac{A_{3}\left(A_{3}^{2}+4 \Omega_{3}^{2}+4 \Delta_{3}^{2}\right)}{\left(A_{3}^{2}+2 \Omega_{3}^{2}+4 \Delta_{3}^{2}\right)^{2}}\right. \\
& \left.-4 \operatorname{Im} C_{3} \frac{\Delta_{3}\left(A_{3}^{2}+4 \Omega_{3}^{2}+4 \Delta_{3}^{2}\right)}{\left(A_{3}^{2}+2 \Omega_{3}^{2}+4 \Delta_{3}^{2}\right)^{2}}\right]
\end{aligned}
$$

to first order in $C_{3}$. While for $p_{01}$ and $p_{10}$ this is also the exact result to all orders, the higher order terms for the other four transitions are too complicated to be given here. The zeroth order terms in equations (3.84) are those one would expect for independent atoms (namely the rates $p_{10}$ and $p_{01}$ for a single $\mathrm{V}$ system multiplied by a factor 1, 2, or 3). For the first order terms it is important to note that the single systems interact via $C_{3}$ only if they are in a bright period. Therefore the rates $p_{01}$ 
and $p_{10}$ are independent of $C_{3}$ while $p_{12}$ and $p_{21}$ have the same first order term as the corresponding rates for two $\mathrm{V}$ systems (in the intensity period $I_{2}$ the three $\mathrm{V}$ systems behave like two $\mathrm{V}$ systems in the period $I_{2}$ plus an additional non-interacting system). In the rates $p_{23}$ and $p_{32}$ the first order term is just twice the first order term of $p_{21}$ and $p_{12}$. This surprising property is due to the simplicity of the quasisteady state $\rho_{\mathrm{ss}, 3}$, namely all diagonal elements of this state have the same first order dependence. Figure 3.9 shows the transition rate $p_{32}$ for three $\mathrm{V}$ systems to first and to second order in $C_{3}$. The first order rate becomes negative for distances of about one half to three quarters of a wavelength of the strong transition. By looking at the second order rate one can see that this is an artifact of the approximation. The rate with the dipole interaction included shows deviations of up to $100 \%$ from the rate for non-interacting atoms for distances of somewhat more than a wavelength $\lambda_{3}$ and still about $10 \%$ for interatomic distances of about $10 \lambda_{3}$. It is realistic that an effect of this size could be observed experimentally, although one has to keep in mind that it refers to an ideal configuration of the ions in the trap and is smaller for other configurations.

The transition rates for three dipole interacting D systems are given by

$$
\begin{aligned}
& p_{01}=3 A_{1}, \quad p_{12}=2 A_{1}, \quad p_{23}=A_{1}, \\
& p_{10}=\frac{A_{2} \Omega_{3}^{2}}{A_{3}^{2}+2 \Omega_{3}^{2}}
\end{aligned}
$$

and

$$
\begin{aligned}
p_{21} & =2 \frac{A_{2} \Omega_{3}^{2}\left(A_{3}^{2}+2 \Omega_{3}^{2}\right)}{\left(A_{3}^{2}+2 \Omega_{3}^{2}\right)^{2}+A_{3}^{2} B} \\
& =\frac{2 A_{2} \Omega_{3}^{2}}{A_{3}^{2}+2 \Omega_{3}^{2}}\left[1-2 \operatorname{Re} C_{3} \frac{A_{3}^{3}}{\left(A_{3}^{2}+2 \Omega_{3}^{2}\right)^{2}}\right]+\mathcal{O}\left(C_{3}^{2}\right), \\
p_{32} & =\frac{3 A_{2} \Omega_{3}^{2}\left[\left(A_{3}^{2}+2 \Omega_{3}^{2}\right)^{2}+A_{3}^{2} B\right]}{\left(A_{3}^{2}+2 \Omega_{3}^{2}\right)\left[\left(A_{3}^{2}+2 \Omega_{3}^{2}\right)^{2}+3 A_{3}^{2} B\right]+2\left(A_{3}^{2}\right)\left[\left|C_{3}\right|^{2}\left|A_{3}+C_{3}\right|^{2}+B^{2}\right]} \\
& =\frac{3 A_{2} \Omega_{3}^{2}}{A_{3}^{2}+2 \Omega_{3}^{2}}\left[1-4 \operatorname{Re} C_{3} \frac{A_{3}^{3}}{\left(A_{3}^{2}+2 \Omega_{3}^{2}\right)^{2}}\right]+\mathcal{O}\left(C_{3}^{2}\right),
\end{aligned}
$$

with $B=\left|C_{3}\right|^{2}+2 A_{3} \operatorname{Re} C_{3}$. For simplicity, only the rates with $\Delta_{3}=0$ are given. The transition rates with detuning are completely analogous to the case of two atoms.

Compared to two D systems the transition rates show the same behavior as explained above for the three $\mathrm{V}$ systems. This is not surprising as the quasi-steady states are identical and as the $\mathrm{D}$ systems also only interact via $C_{3}$ when they are in a bright period. Figure 3.10 shows the exact transition rate $p_{32}$ compared to the interaction free case. For distances of about a wavelength, $p_{32}$ deviates up to $75 \%$ from the rate without interaction. The first peak at about 0.7 wavelengths even reaches a maximum of seven times the rate for independent atoms. For such small distances, however, one would have to check the validity of the model (namely, that 
in a particular intensity period most of the population is in a specific subspace). Also one has to keep in mind that all the experiments cited here were performed at greater ion distances. As for two D systems large cooperative effects occur for weak driving and decrease with increasing Rabi frequency. For strong driving $\left(\Omega_{3}>A_{3}\right)$ the effects are negligible in accordance with the experimental results for the $\mathrm{Hg}^{+}$ ions.

\subsubsection{Double and triple jump rate}

As for two atoms one can evaluate the double jump rate from the transition rates given above. Since for three atoms there are now four periods of different intensity it consists of four contributions

$$
n_{\mathrm{DJ}}=n_{\mathrm{DJ}}^{20}+n_{\mathrm{DJ}}^{31}+n_{\mathrm{DJ}}^{13}+n_{\mathrm{DJ}}^{02} .
$$

In addition there is also the possibility of triple jumps, i.e. a change in intensity within the time window $T_{\mathrm{W}}$ from zero to the maximum value or vice versa.

$$
n_{\mathrm{TJ}}=n_{\mathrm{TJ}}^{30}+n_{\mathrm{TJ}}^{03} .
$$

The calculation of the double jump rate is analogous to the two atom case. The contributions starting in periods $I_{0}$ and $I_{3}$ are given by

$$
n_{\mathrm{DJ}}^{02}=n_{0} \frac{p_{12}}{p_{10}+p_{12}}\left(1-\mathrm{e}^{-\left(p_{10}+p_{12}\right) T_{\mathrm{W}}}\right), \quad n_{\mathrm{DJ}}^{31}=n_{3} \frac{p_{21}}{p_{21}+p_{23}}\left(1-\mathrm{e}^{-\left(p_{21}+p_{23}\right) T_{\mathrm{W}}}\right) .
$$

The other two contributions

$$
n_{\mathrm{DJ}}^{13}=n_{1} \frac{p_{12}}{p_{10}+p_{12}} \frac{p_{23}}{p_{21}+p_{23}}\left(1-\mathrm{e}^{-\left(p_{21}+p_{23}\right) T_{\mathrm{W}}}\right)
$$

and

$$
n_{\mathrm{DJ}}^{20}=n_{2} \frac{p_{21}}{p_{21}+p_{23}} \frac{p_{10}}{p_{10}+p_{12}}\left(1-\mathrm{e}^{-\left(p_{10}+p_{12}\right) T_{\mathrm{W}}}\right)
$$

each have an additional branching ratio since the initial intensity periods can be followed by periods with higher or lower intensity. With the relations

$$
\begin{aligned}
& n_{0}=\frac{p_{10}}{p_{10}+p_{12}} n_{1}, \quad n_{3}=\frac{p_{23}}{p_{21}+p_{23}} n_{2}, \\
& n_{2}=\frac{p_{12}}{p_{10}+p_{12}} n_{1}+n_{3}, \quad \text { and } \quad n_{1}=n_{0}+\frac{p_{21}}{p_{21}+p_{23}} n_{2} \text {, }
\end{aligned}
$$

the rates can be simplified to

$$
n_{\mathrm{DJ}}^{02}=n_{\mathrm{DJ}}^{20}=n_{1} \frac{p_{10} p_{12}}{\left(p_{10}+p_{12}\right)^{2}}\left(1-\mathrm{e}^{-\left(p_{10}+p_{12}\right) T_{\mathrm{W}}}\right)
$$



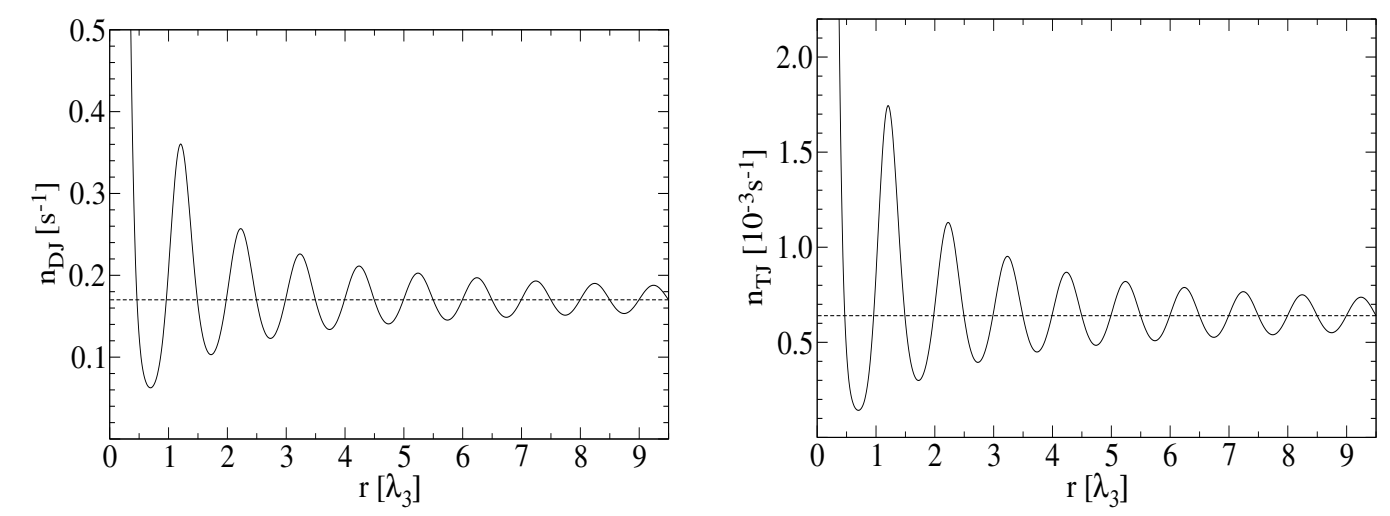

Figure 3.11: Double jump rate $n_{D J}$ and triple jump rate $n_{T J}$ for three dipoleinteracting $V$ systems. Solid line: up to second order in $C_{3}$. Dashed line: independent systems. Time window $T_{W}=10^{-3} \mathrm{~s}$. Other parameter values as in Figure 3.9.

and

$$
n_{\mathrm{DJ}}^{13}=n_{\mathrm{DJ}}^{31}=n_{1} \frac{p_{12} p_{23}}{\left(p_{21}+p_{23}\right)\left(p_{10}+p_{12}\right)}\left(1-\mathrm{e}^{-\left(p_{21}+p_{23}\right) T_{\mathrm{W}}}\right) .
$$

Using the relations

$$
T_{0}=\frac{1}{p_{01}}, \quad T_{1}=\frac{1}{p_{10}+p_{12}}, \quad T_{2}=\frac{1}{p_{21}+p_{23}}, \quad T_{3}=\frac{1}{p_{32}}, \quad \sum_{i=0}^{3} n_{i} T_{i}=1
$$

for the mean durations of the intensity periods and expanding the exponential one finally obtains

$$
n_{\mathrm{DJ}}=2 \frac{p_{01} p_{21} p_{32}\left(p_{01}+p_{12}\right)}{p_{21} p_{32}\left(p_{01}+p_{10}\right)+P_{01} p_{12}\left(p_{23}+p_{32}\right)} T_{\mathrm{W}}
$$

as the double jump rate for three of either three-level systems.

The calculation of the triple jump rate is similar. The rate for a jump from period $I_{3}$ to period $I_{0}$ is given by

$$
n_{\mathrm{TJ}}^{30}=n_{3} \frac{p_{10} p_{21}}{\left(p_{10}+p_{12}\right)\left(p_{21}+p_{23}\right)}\left(1-\mathrm{e}^{-\left(p_{21}+p_{23}\right) T_{\mathrm{W}}}\right)\left(1-\mathrm{e}^{-\left(p_{10}+p_{12}\right) T_{\mathrm{W}}}\right)
$$

with the appropriate branching ratios for downward jumps and with the probabilities for the periods $I_{2}$ and $I_{1}$ to be shorter than $T_{\mathrm{W}}$ given by

$$
p_{T_{2}<T_{\mathrm{W}}}=1-\mathrm{e}^{-\left(p_{21}+p_{23}\right) T_{\mathrm{W}}} \quad p_{T_{1}<T_{\mathrm{W}}}=1-\mathrm{e}^{-\left(p_{10}+p_{12}\right) T_{\mathrm{W}}} .
$$

Analogously one has

$$
n_{\mathrm{TJ}}^{03}=n_{0} \frac{p_{12} p_{23}}{\left(p_{10}+p_{12}\right)\left(p_{21}+p_{23}\right)}\left(1-\mathrm{e}^{-\left(p_{21}+p_{23}\right) T_{\mathrm{W}}}\right)\left(1-\mathrm{e}^{-\left(p_{10}+p_{12}\right) T_{\mathrm{W}}}\right) .
$$



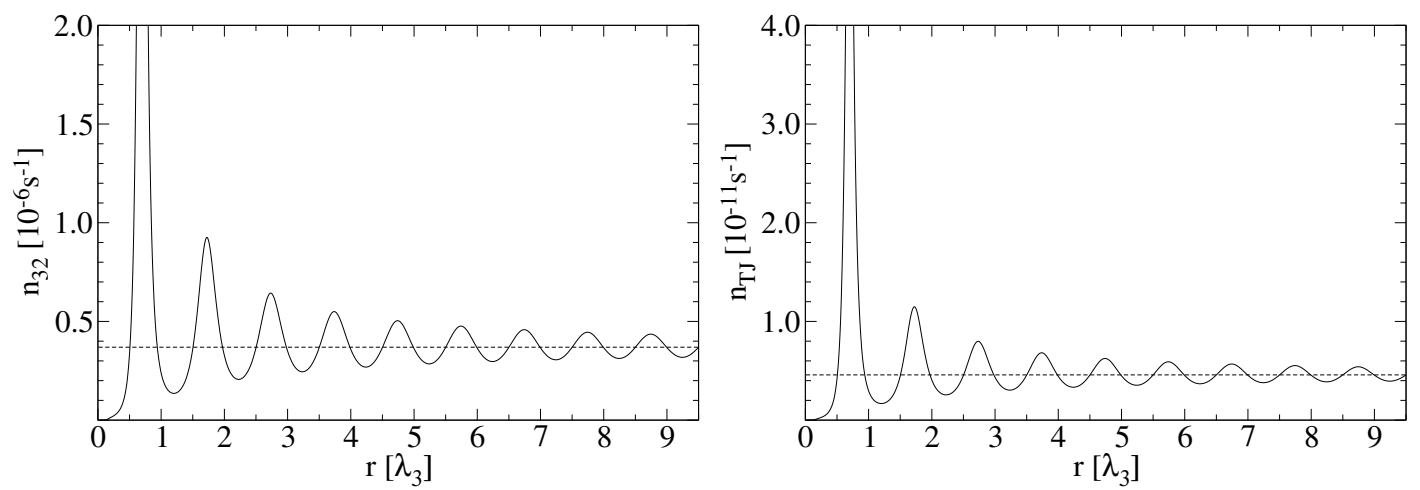

Figure 3.12: Double jump rate $n_{D J}$ and triple jump rate $n_{T J}$ for three dipoleinteracting $D$ systems. Dashed line: independent systems. Time window $T_{W}=$ $5 \times 10^{-3}$ s. Other parameter values as in Figure 3.10.

Expanding the exponentials in both contributions yields

$$
n_{\mathrm{TJ}}^{30}=n_{3} p_{10} p_{21} T_{\mathrm{W}}^{2}, \quad \text { and } \quad n_{\mathrm{TJ}}^{03}=n_{0} p_{12} p_{23} T_{\mathrm{W}}^{2} .
$$

Using the relations (3.89) and (3.91) one finds that

$$
n_{\mathrm{TJ}}^{30}=n_{\mathrm{TJ}}^{03}=n_{1} \frac{p_{10} p_{12} p_{23}}{p_{10}+p_{12}} T_{\mathrm{W}}^{2}
$$

and finally

$$
n_{\mathrm{TJ}}=2 \frac{p_{01} p_{10} p_{12} p_{21} p_{23} p_{32}}{p_{21} p_{32}\left(p_{01}+p_{10}\right)+p_{01} p_{12}\left(p_{23}+p_{32}\right)} T_{\mathrm{W}}^{2}
$$

Note that the defining time window $T_{\mathrm{W}}$ enters quadratically in this case. Figures 3.11 and 3.12 show plots of $n_{\mathrm{DJ}}$ and $n_{\mathrm{TJ}}$ for the $\mathrm{V}$ systems and the $\mathrm{D}$ systems, respectively. For the $\mathrm{D}$ systems the exact values for the $p_{i j}$ are used whereas for the $\mathrm{V}$ systems only the expanded expressions up to second order in $C_{3}$ are used since $p_{23}$ and $p_{23}$ could not be calculated exactly for the $\mathrm{V}$ systems. For the $\mathrm{V}$ systems there are cooperative effects of up to $110 \%$ for the double jump rate $n_{\text {DJ }}$ and $170 \%$ for the triple jump rate $n_{\mathrm{TJ}}$ for distances of somewhat more than a wavelength of the strong transition. For the same distance range the D system shows cooperative effects of up to $150 \%$ for both $n_{\mathrm{DJ}}$ and $n_{\mathrm{TJ}}$. The first peak at three quarters of a wavelength reaches 16 times the value for independent systems for both rates. For distances of about 10 wavelengths cooperative effects of $15 \%$ are still present for both systems. In the case of the D system, which models the level configuration of the $\mathrm{Hg}^{+}$ions used in the experiments of Reference [23, 45], large cooperative effects only appear if the Rabi frequency $\Omega_{3}$ is smaller than the Einstein coefficient $A_{3}$. So, for the experimental parameters (i.e. $\Omega_{3}>A_{3}$ and $r / \lambda_{3} \approx 15$ ) the effects are negligible, in agreement with the experimental results. 


\subsubsection{Simplified approach: Treatment of three four-level systems}

Due to the increased number of levels involved, a calculation of a the transition rates for three dipole-interacting four-level systems would be, although in principal possible with the methods introduced above, even more laborious than for three three-level systems. It is however possible to read off the transition rates without having to carry out the full calculation. One only needs the steady states of the corresponding subsystems. In the following this simpler approach will be presented and verified by applying it to examples for which the transition rates have already been calculated in the previous sections.

In order to illustrate the underlying mechanism the Bloch equation approach for a single D system will now be recapitulated and given explicitly. The conditional Hamiltonian of this system is given by

$$
H_{\text {cond }}=\frac{\hbar}{2 \mathrm{i}}\left[\left(A_{2}+A_{3}\right)|3\rangle\left\langle 3\left|+A_{1}\right| 2\right\rangle\langle 2|\right]+\frac{\hbar \Omega_{3}}{2}[|1\rangle\langle 3|+| 3\rangle\langle 1|],
$$

where the detuning has been neglected for simplicity. The reset state is given by

$$
\mathscr{R}(\rho)=A_{1}|1\rangle\langle 2|\rho| 2\rangle\left\langle 1\left|+A_{2}\right| 2\right\rangle\langle 3|\rho| 3\rangle\left\langle 2\left|+A_{3}\right| 1\right\rangle\langle 3|\rho| 3\rangle\langle 1| .
$$

Defining, as before, the Liouvillean of the system as

$$
\dot{\rho}=\mathscr{L} \rho=-\frac{\mathrm{i}}{\hbar}\left[H_{\text {cond }} \rho-\rho H_{\text {cond }}^{\dagger}\right]+\mathscr{R}(\rho)
$$

and splitting it into two parts as

$$
\mathscr{L}=\mathscr{L}_{0}\left(A_{3}, \Omega_{3}\right)+\mathscr{L}_{1}\left(A_{1}, A_{2}\right)
$$

the density matrix at time $t+\Delta t$ is given by

$$
\begin{aligned}
\rho\left(t+\Delta t, \rho_{i}\right) & =\rho_{\mathrm{ss}, i}+\int_{0}^{\Delta t} \mathrm{~d} \tau \mathrm{e}^{\mathscr{L}_{0} \tau} \mathscr{L}_{1} \rho_{\mathrm{ss}, i} \\
& =\rho_{\mathrm{ss}, i}+\sum_{j=1}^{2} \alpha_{i j} \rho_{\mathrm{ss}, j} \Delta t+\int_{0}^{\Delta t} \mathrm{~d} \tau \mathrm{e}^{\mathscr{L}_{0} \tau} \mathscr{L}_{1} \tilde{\rho} .
\end{aligned}
$$

If the small optical parameters $A_{1}, A_{2}$ are neglected the system splits into independent subspaces. They are given by

$$
\mathscr{S}_{0}=\{|2\rangle\}, \quad \mathscr{S}_{1}=\{|1\rangle,|3\rangle\}
$$

The steady states in these subspaces are given by

$$
\rho_{\mathrm{ss}, 0}=|2\rangle\langle 2|,
$$




$$
\rho_{\mathrm{ss}, 1}=\frac{1}{A_{3}^{2}+2 \Omega_{3}^{2}}\left[\left(A_{3}^{2}+\Omega_{3}^{2}\right)|1\rangle\left\langle 1\left|+\Omega_{3}^{2}\right| 3\right\rangle\langle 3|+\mathrm{i} A_{3} \Omega_{3}(|1\rangle\langle 3|-| 3\rangle\langle 1|)\right]
$$

for the dark and the bright period respectively. One therefore has

$$
\begin{aligned}
& \mathscr{L}_{1} \rho_{\mathrm{ss}, 0}=-A_{1}|2\rangle\left\langle 2\left|+A_{1}\right| 1\right\rangle\langle 1| \\
& \mathscr{L}_{1} \rho_{\mathrm{ss}, 1}=A_{2} \frac{\Omega_{3}^{2}}{A_{3}^{2}+2 \Omega_{3}^{2}}(|2\rangle\langle 2|-| 3\rangle\langle 3|)-\frac{\mathrm{i} A_{2}}{2} \frac{A_{3} \Omega_{3}}{A_{3}^{2}+2 \Omega_{3}^{2}}(|1\rangle\langle 3|-| 3\rangle\langle 1|)
\end{aligned}
$$

With the dual steady states

$$
\rho_{\mathrm{ss}}^{0}=|2\rangle\langle 2|, \quad \text { and } \quad \rho_{\mathrm{ss}}^{1}=|1\rangle\langle 1|+| 3\rangle\langle 3|
$$

the transition rates are then calculated to be

$$
p_{01}=\alpha_{01}=\operatorname{Tr}\left(\rho_{\mathrm{ss}}^{1 \dagger} \mathscr{L}_{1} \rho_{\mathrm{ss}, 0}\right)=A_{1}
$$

and

$$
p_{10}=\alpha_{10}=\operatorname{Tr}\left(\rho_{\mathrm{ss}}^{0 \dagger} \mathscr{L}_{1} \rho_{\mathrm{ss}, 1}\right)=\frac{A_{2} \Omega_{3}^{2}}{A_{3}^{2}+2 \Omega_{3}^{2}}
$$

By looking at equation (3.104) one realizes that this latter step in the calculation although formally more satisfactory was actually not necessary in order to gain the final result. The transition rates are already present as prefactors to some of the density matrix elements. In fact, $\mathscr{L}_{1}$ can be interpreted as a transition operator which gives the transitions due to the weak Einstein coefficients. Multiplying it to some state of the system yields the density matrix elements which are modified by the weak decays multiplied by the corresponding decay rates, positive for density matrix element which gain population and negative for those which loose population due to the decay. In the case in which one started with $\rho_{\mathrm{ss}, 0}=|2\rangle\langle 2|$ one therefore has a term $-A_{1}|2\rangle\langle 2|$ taking into account the loss of population of level $|2\rangle$ and a term $A_{1}|1\rangle\langle 1|$ for the corresponding gain of population in the ground state. When starting with $\rho_{\mathrm{ss}, 1}$ the Einstein coefficient $A_{2}$ for the decay from $|3\rangle$ to $|2\rangle$ has to be multiplied with the additional factor $\frac{\Omega_{3}^{2}}{A_{3}^{2}+2 \Omega_{3}^{2}}$ for the steady state population of level $|3\rangle$. The last two terms in (3.104b) are due to the decay of the coherences between $|1\rangle$ and $|3\rangle$.

From these considerations one is lead to a simple scheme for the evaluation of the transition rates. First one has to identify the different independent subspaces for vanishing weak decay rates and calculate the steady states in these subspaces. For a single D system these are the states $\rho_{\mathrm{ss}, 0}$ and $\rho_{\mathrm{ss}, 1}$ for the subsystems associated with the dark and bright period, respectively. By looking at the level scheme one can then determine the possible decay channels between the subsystems, a decay by $A_{2}$ from $|3\rangle$ to $|2\rangle$ and a decay by $A_{1}$ from $|2\rangle$ to $|1\rangle$ in the case of one $\mathrm{D}$ system. 
The transition rates are then given by the decay rate multiplied by the steady state population of the decaying level.

Physically this is quite intuitive. The transition rates are given by the corresponding decay rates multiplied by the mean occupation probabilities of the levels involved.

The question is now if this approach can be extended to more complicated systems, especially to dipole-interacting D systems and to the four-level system for the description of $\mathrm{Ba}^{+}$. This is indeed possible. For two dipole-interacting D systems for example the possible decays can be read off in Figure 3.5(b) and the steady states are given by equations (3.34). The easiest case is the transition rate $p_{01}$. Here the transitions involved are from $\left|e_{2}\right\rangle$ to $\left|s_{12}\right\rangle$ and $\left|a_{12}\right\rangle$. The corresponding decay rates are $A_{1}+\operatorname{Re} C_{1}$ and $A_{1}-\operatorname{Re} C_{1}$, respectively. The steady state population of $\left|e_{2}\right\rangle$ is unity, so the transition rate is $p_{01}=2 A_{1}$ in agreement with the previous result. The other transition rates are a bit more complicated. For $p_{10}$ one has to take into account the decays from $\left|s_{23}\right\rangle$ and $\left|a_{23}\right\rangle$ to $\left|e_{2}\right\rangle$, for $p_{12}$ the decays from $\left|s_{23}\right\rangle$ and $\left|a_{23}\right\rangle$ to $\left|s_{13}\right\rangle$ and from $\left|s_{12}\right\rangle$ and $\left|a_{12}\right\rangle$ to $|g\rangle$, and for $p_{21}$ the decays from $\left|e_{3}\right\rangle$ to $\left|s_{23}\right\rangle$ and $\left|a_{23}\right\rangle$ and from $\left|s_{13}\right\rangle$ and $\left|a_{13}\right\rangle$ to $\left|s_{12}\right\rangle$ and $\left|a_{12}\right\rangle$. By multiplying for each decay the decay rate by the steady state population of the initial level and adding up the different contributions one obtains the results for the transition rates calculated in Section 3.2.2. The same is also true for three dipole-interacting D systems.

An application of this method to the four-level system describing $\mathrm{Ba}^{+}$is also possible. For the transition from a bright to a dark period one has to use the product of the incoherent transition rate $W$ with the branching ratio $A_{2} /\left(A_{2}+A_{4}\right)$ for a decay from state $|4\rangle$ to state $|2\rangle$ instead of a single Einstein coefficient. Then everything works as in the case of the D systems and one confirms the results for one and two four-level systems already known from the Bloch equation approach.

Consequently it is also possible to obtain the transition rates for three four-level systems which would be rather involved to do with the Bloch equation approach. The steady states are already known from the calculations for three three-level systems. As for three three-level systems one can use a symmetrized basis analogous to the Dicke basis for two atoms. This leads to the states

$$
\begin{aligned}
\left|s_{i j k}\right\rangle=\frac{1}{\sqrt{6}} & (|i\rangle|j\rangle|k\rangle+|j\rangle|k\rangle|i\rangle+|k\rangle|i\rangle|j\rangle \\
& +|i\rangle|k\rangle|j\rangle+|j\rangle|i\rangle|k\rangle+|k\rangle|j\rangle|i\rangle), \\
\left|a_{i j k}\right\rangle=\frac{1}{\sqrt{6}}( & (|i\rangle|j\rangle|k\rangle+|j\rangle|k\rangle|i\rangle+|k\rangle|i\rangle|j\rangle \\
& -|i\rangle|k\rangle|j\rangle-|j\rangle|i\rangle|k\rangle-|k\rangle|j\rangle|i\rangle),
\end{aligned}
$$




$$
\begin{aligned}
&\left|b_{i j k}\right\rangle= \frac{1}{\sqrt{12}}(2|i\rangle|j\rangle|k\rangle-|j\rangle|k\rangle|i\rangle-|k\rangle|i\rangle|j\rangle \\
&+2|i\rangle|k\rangle|j\rangle-|j\rangle|i\rangle|k\rangle-|k\rangle|j\rangle|i\rangle), \\
&\left|c_{i j k}\right\rangle= \frac{1}{2}(|j\rangle|k\rangle|i\rangle-|k\rangle|i\rangle|j\rangle \\
&-|j\rangle|i\rangle|k\rangle+|k\rangle|j\rangle|i\rangle), \\
&\left|d_{i j k}\right\rangle= \frac{1}{\sqrt{12}}(2|i\rangle|j\rangle|k\rangle-|j\rangle|k\rangle|i\rangle-|k\rangle|i\rangle|j\rangle \\
&\quad-2|i\rangle|k\rangle|j\rangle+|j\rangle|i\rangle|k\rangle+|k\rangle|j\rangle|i\rangle), \\
&\left|e_{i j k}\right\rangle=\frac{1}{2}(|j\rangle|k\rangle|i\rangle-|k\rangle|i\rangle|j\rangle \\
&+|j\rangle|i\rangle|k\rangle-|k\rangle|j\rangle|i\rangle),
\end{aligned}
$$

$i<j<k ; i, j, k=1, \ldots, 4$, in the case where all three atoms are in different states. For the remaining states one gets for $i, j=1, \ldots, 4, i \neq j$,

$$
\begin{aligned}
\left|s_{i j j}\right\rangle & =\frac{1}{\sqrt{3}}(|i\rangle|j\rangle|j\rangle+|j\rangle|j\rangle|i\rangle+|j\rangle|i\rangle|j\rangle), \\
\left|b_{i j j}\right\rangle & =\frac{1}{\sqrt{6}}(2|i\rangle|j\rangle|j\rangle-|j\rangle|j\rangle|i\rangle-|j\rangle|i\rangle|j\rangle), \\
\left|c_{i j j}\right\rangle & =\frac{1}{\sqrt{2}}(|j\rangle|j\rangle|i\rangle-|j\rangle|i\rangle|j\rangle),
\end{aligned}
$$

if two atoms are in the same state and

$$
|g\rangle=|1\rangle|1\rangle|1\rangle, \quad\left|e_{i}\right\rangle=|i\rangle|i\rangle|i\rangle \quad \text { for } \quad i=2,3,4
$$

if all three atoms are in the same state. The quasi-steady states for intensity periods $I_{0}$ to $I_{2}$ are, by symmetry, given by

$$
\begin{aligned}
\rho_{\mathrm{ss}, 0}= & \left|e_{2}\right\rangle\left\langle e_{2}\right|, \\
\rho_{\mathrm{ss}, 1}= & \frac{1}{3}\left\{\rho_{\mathrm{ss}}^{1 \mathrm{D}} \otimes|2\rangle_{22}\langle 2|\otimes| 2\rangle_{33}\langle 2|\right. \\
& \left.\quad+|2\rangle_{11}\left\langle 2\left|\otimes \rho_{\mathrm{ss}}^{1 \mathrm{D}} \otimes\right| 2\right\rangle_{33}\langle 2|+| 2\rangle_{11}\langle 2|\otimes| 2\rangle_{22}\langle 2| \otimes \rho_{\mathrm{ss}}^{1 \mathrm{D}}\right\}, \\
\rho_{\mathrm{ss}, 2}= & \frac{1}{3} \sum_{i=1}^{3} \rho_{\mathrm{ss}, 2}^{2 \mathrm{D}} \otimes|2\rangle_{i i}\langle 2|,
\end{aligned}
$$

where $\rho_{\mathrm{ss}}^{1 \mathrm{D}}$ is the quasi-steady state of one D system in the $\{|1\rangle,|3\rangle\}$ subspace and $\rho_{\mathrm{ss}, 2}^{2 \mathrm{D}}$ is the quasi-steady state in the subspace corresponding to double intensity of 
two D systems. The state $\rho_{\mathrm{ss}, 3}$ is rather complicated. Therefore only the populations of the relevant levels will be given, i.e.

$$
\begin{aligned}
\left\langle g\left|\rho_{\mathrm{ss}, 3}\right| g\right\rangle=\frac{1}{N}[ & \left(A_{3}^{2}+\Omega_{3}^{2}+4 \Delta_{3}^{2}\right)\left[\left(A_{3}^{2}+\Omega_{3}^{2}+4 \Delta_{3}^{2}\right)^{2}+3\left(A_{3}^{2}+4 \Delta_{3}^{2}\right) B\right] \\
& \left.+2\left(A_{3}+4 \Delta_{3}^{2}\right)\left[\left|C_{3}\right|^{2}\left|A_{3}-2 \mathrm{i} \Delta_{3}+C_{3}\right|^{2}+B^{2}\right]\right], \\
\left\langle s_{113}\left|\rho_{\mathrm{ss}, 3}\right| s_{113}\right\rangle= & \frac{\Omega_{3}^{2}}{N}\left[\left(A_{3}^{2}+\Omega_{3}^{2}+4 \Delta_{3}^{2}\right)\left(3 A_{3}^{2}+\Omega_{3}^{2}+12 \Delta_{3}^{2}\right)+3\left(A_{3}^{2}+4 \Delta_{3}^{2}\right) B\right] \\
\left\langle b_{113}\left|\rho_{\mathrm{ss}, 3}\right| b_{113}\right\rangle= & \left\langle c_{113}\left|\rho_{\mathrm{ss}, 3}\right| c_{113}\right\rangle=\frac{\Omega_{3}^{4}}{N}\left(A_{3}^{2}+\Omega_{3}^{2}+4 \Delta_{3}^{2}\right) \\
\left\langle s_{133}\left|\rho_{\mathrm{ss}, 3}\right| s_{133}\right\rangle= & \frac{\Omega_{3}^{4}}{N}\left(3 A_{3}^{2}+\Omega_{3}^{2}+12 \Delta_{3}^{2}\right) \\
\left\langle e_{3}\left|\rho_{\mathrm{ss}, 3}\right| e_{3}\right\rangle= & \left\langle b_{133}\left|\rho_{\mathrm{ss}, 3}\right| b_{133}\right\rangle=\left\langle c_{133}\left|\rho_{\mathrm{ss}, 3}\right| c_{133}\right\rangle=\frac{\Omega_{3}^{6}}{N}
\end{aligned}
$$

with

$$
\begin{aligned}
N=\left\{( A _ { 3 } ^ { 2 } + 2 \Omega _ { 3 } ^ { 2 } + 4 \Delta _ { 3 } ^ { 2 } ) \left[\left(A_{3}^{2}+\right.\right.\right. & \left.\left.2 \Omega_{3}^{2}+4 \Delta_{3}^{2}\right)^{2}+3\left(A_{3}^{2}+4 \Delta_{3}^{2}\right) B\right] \\
& \left.+2\left(A_{3}+4 \Delta_{3}^{2}\right)\left[\left|C_{3}\right|^{2}\left|A_{3}-2 \mathrm{i} \Delta_{3}+C_{3}\right|^{2}+B^{2}\right]\right\}
\end{aligned}
$$

and

$$
B=\left|C_{3}\right|^{2}+2 A_{3} \operatorname{Re} C_{3}-4 \Delta_{3} \operatorname{Im} C_{3} .
$$

Now the procedure is the same as described above for two D systems and one obtains

$$
p_{01}=3 A_{1}, \quad p_{12}=2 A_{1}, \quad p_{23}=A_{1},
$$

and

$$
\begin{aligned}
p_{10} & =\frac{A_{2} W\left(A_{3}^{2}+\Omega_{3}^{2}\right)}{\left(A_{2}+A_{4}\right)\left[A_{3}^{2}+2 \Omega_{3}^{2}\right]}, \\
p_{21} & =2 \frac{A_{2} W}{A_{2}+A_{4}} \frac{\left(A_{3}^{2}+\Omega_{3}^{2}\right)\left(A_{3}^{2}+2 \Omega_{3}^{2}\right)+A_{3}^{2}\left(\left|C_{3}\right|^{2}+2 A_{3} \operatorname{Re} C_{3}\right)}{\left(A_{3}^{2}+2 \Omega_{3}^{2}\right)^{2}+A_{3}^{2}\left(\left|C_{3}\right|^{2}+2 A_{3} \operatorname{Re} C_{3}\right)} \\
& =2 \frac{A_{2} W}{A_{2}+A_{4}}\left[\frac{A_{3}^{2}+\Omega_{3}^{2}}{A_{3}^{2}+2 \Omega_{3}^{2}}+2 \operatorname{Re} C_{3} \frac{A_{3}^{3} \Omega_{3}^{2}}{\left[A_{3}^{2}+2 \Omega_{3}^{2}\right]^{3}}\right]+\mathcal{O}\left(C_{3}^{2}\right) . \\
p_{32} & =3 \frac{A_{2} W}{A_{2}+A_{4}} \frac{\left(A_{3}^{2}+\Omega_{3}^{2}\right)\left[\left(A_{3}^{2}+2 \Omega_{3}^{2}\right)^{2}+3 A_{3}^{2} B\right]+2 A_{3}^{2}\left[\left|C_{3}\right|^{2}\left|A_{3}+C_{3}\right|^{2}+B \Omega^{2}+B^{2}\right]}{\left(A_{3}^{2}+2 \Omega_{3}^{2}\right)\left[\left(A_{3}^{2}+2 \Omega_{3}^{2}\right)^{2}+3 A_{3}^{2} B\right]+2 A_{3}^{2}\left[\left|C_{3}\right|^{2}\left|A_{3}+C_{3}\right|^{2}+B^{2}\right]} \\
& =3 \frac{A_{2} W}{A_{2}+A_{4}}\left[\frac{A_{3}^{2}+\Omega_{3}^{2}}{A_{3}^{2}+2 \Omega_{3}^{2}}+4 \operatorname{Re} C_{3} \frac{A_{3}^{3} \Omega_{3}^{2}}{\left[A_{3}^{2}+2 \Omega_{3}^{2}\right]^{3}}\right]+\mathcal{O}\left(C_{3}^{2}\right)
\end{aligned}
$$



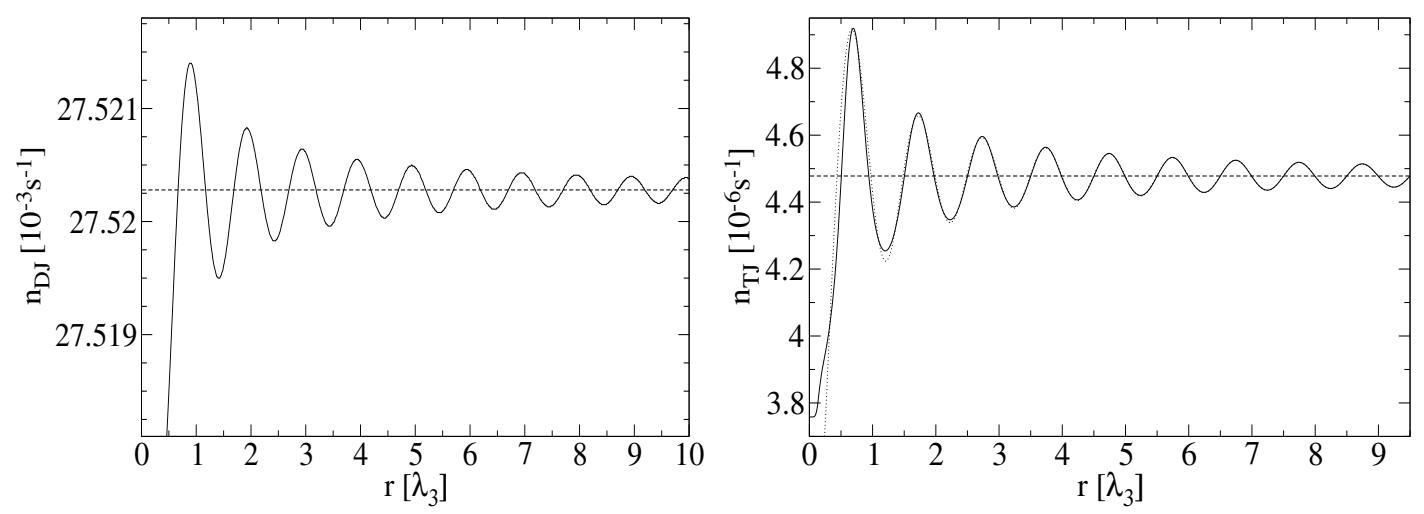

Figure 3.13: Double jump rate $n_{D J}$ and triple jump rate $n_{T J}$ for three dipoleinteracting four-level systems with experimental parameter values of reference [3]. Solid line: exact results, dashed line: independent systems. In the graph for $n_{T J}$ $\Delta_{3}=0$ and $\Omega_{3}=\frac{1}{2} \sqrt{\sqrt{5}-1} A_{3}$ are chosen for maximal effects and the dotted line shows the result up to first order in $C_{3}$.

as transition rates up to first order in $C_{3}$. The exact results including detuning are given in Appendix B. The approximations to first order in $C_{3}$ have the same structure as in the other cases. In Figure 3.13 plots of $n_{\mathrm{DJ}}$ and $n_{\mathrm{TJ}}$ are shown. The double jump rate is displayed for the experimental parameter values of Reference [3]. The effects of the dipole-dipole interaction are negligibly small in particular for experimental distances of about ten times the wavelength $\lambda_{3}$ of the strong transition. Without detuning $\Delta_{3}$, maximal cooperative effects are obtained for $\Omega_{3}=\frac{1}{2} \sqrt{\sqrt{5}-1} A_{3}$. This case is shown in Figure 3.13 for the triple jump rate $n_{\mathrm{TJ}}$. For inter-atomic distances larger than one wavelength $\lambda_{3}$ of the strong transition cooperative effects are less than $5 \%$ and as before they are rapidly decreasing for larger distances. For nonzero detuning the maximally achievable effects have about the same value. Also one has to bear in mind that, as in Reference [48], this result has to be seen as an upper limit for all possible configurations in the trap. Large cooperative effects, i.e. enhancements of the double and triple jump rate by several orders of magnitude, can therefore not be explained by the dipole-dipole interaction. Furthermore one sees that the first order results of equation (3.113) are a very good approximation to the exact transition rates given in Appendix B.

\section{V system and similar level schemes}

From the previous results the question arises whether the method presented here is also applicable to level systems like the $\mathrm{V}$ system, i.e. systems in which the transition between different bright and dark periods results from a coherent excitation. It turns out that for these systems the situation is much more complicated. For a single $\mathrm{V}$ system for example $\mathscr{L}_{1}\left(\Omega_{2}\right)$ contains coherences between the ground state $|1\rangle$ and the meta-stable state $|2\rangle$. Therefore $\mathscr{L}_{1} \rho_{i}$ has no component in the subspace of 
eigenstates of $\mathscr{L}_{0}$ for eigenvalue zero. According to equations (3.30) and (3.31) the state at time $t+\Delta t$ in the Bloch equation approach is thus given by

$$
\rho\left(t+\Delta t, \rho_{0, i}\right)=\rho_{\mathrm{ss}, i}+\left(\epsilon-\mathscr{L}_{0}\right)^{-1} \mathscr{L}_{1} \rho_{\mathrm{ss}, i}
$$

An explicit evaluation of this expression for a single $\mathrm{V}$ system starting with $\rho_{1}$ not only leads to terms proportional to the quasi-steady state population of the ground state but also to terms proportional to the quasi-steady state coherence between ground state and excited state.

The situation gets even more involved for dipole-interacting V systems. Here the term $\left(\epsilon-\mathscr{L}_{0}\right)^{-1}$ gives rise to additional factors which depend in a very complicated way on $C_{3}$. This is in contrast to the $\mathrm{D}$ and the four-level system, for which the $C_{3}$ dependence in the transition rates is solely due to $C_{3}$ dependence of the quasisteady states. The physical reason is that the efficiency of the laser driving is influenced by the dipole interaction, for example via additional detunings. Therefore the mechanism of the quantum jumps is much more complex than for jumps based on spontaneous decay so that the method outlined above is only applicable in the latter case. 


\section{Part II}

\section{Detection of ultra-cold moving atoms}




\section{Introduction}

Atom optics is a recent and growing sub-field of atomic, molecular, and optical physics which aims at exploiting the wave nature of matter. In this vain, one has realized conventional optics experiments with atoms instead of light. Often the role of light and matter with respect to their usual use in conventional optics is simply reversed. More specifically, the influence of laser light on the translational motion of atoms can be utilized to construct optical elements like beam splitters, diffraction gratings, lenses, and so on based on light.

In the second part of this work a model for the measurement of properties of the wave function of laser cooled atoms is developed. The idea to this model is taken from an atom optical experiment by Szriftgiser et al. $[49,50]$. It was part of a series of experiments performed in the group of Dalibard in which an evanescent wave atomic mirror was used to demonstrate a number of atom optical devices like a lens and a phase modulator $[51,52]$. In the particular experiment of interest, the realization of the temporal equivalent of a diffraction experiment was demonstrated. The basic principle of the experiment is the following. A cloud of laser cooled cesium atoms is released from a magneto-optical trap located above the mirror. After two rebounds during which the mirror is switched on for only a short period of time the atoms show a diffraction pattern. This is detected by measuring the number of atoms reflected in a third rebound, again with a short switch-on time of the mirror. Since the atomic gas is very dilute the atoms do not interact with each other and the experiment can be seen as an experiment with single atoms.

The part of the experiment described above most interesting for the present work is the measurement procedure given by the third reflection. It can be used to infer interesting quantum features from the wave functions of the atoms impinging on the mirror. When switched on, the mirror basically cuts out a short part of the atomic wave function, which is reflected. This small section contains information about the value of the wave function at the specific point which hit the mirror at the moment it was switched on. The shorter the switch-on time is the more precise this information is. A model for this procedure will be developed in this work which allows the detailed analysis of the process. The question of how to prepare a suitable wave packet to be detected by the mirror is not considered in this work. The above diffraction experiment can be seen as a way of achieving this technically.

Two distinct measurement set-ups are investigated in the following. First, if the switch-on time of the mirror is kept fixed and the position of the mirror is moved it 
will be shown that one can measure the probability density, i.e. the modulus squared, of the wave function. This can be seen as an interesting result in the field of quantum state estimation. In this area of research one tries to deduce the full information about a given state of a quantum mechanical system from a series of measurements (for a recent review see reference [53]). This has already been achieved for different quantum states of the radiation field [54], for the motional state of an atom in a trap [55], the vibrational state of a molecule [56], and especially has become a standard technique in quantum information processing with trapped ions for the reconstruction of the internal states of the ions $[57,58]$. A partial reconstruction of the motional state of unbound atoms has been achieved experimentally for atomic beams with interferometric methods, namely for the transversal degrees of freedom $[59,60]$ and for the longitudinal degree of freedom [61, 62]. However, for laser cooled atoms released from a trap a quantum state reconstruction of the translational degrees of freedom has not been realized so far. The result presented here can be seen as a step in this direction.

Secondly, if the position of the mirror is kept fixed and the reflection window is shifted in time one may expect from considerations based on classical mechanics to measure the arrival-time distribution of the atoms. In quantum mechanics, however, the notion of arrival-time and of time observables in general is not unambiguous and a still controversially discussed question [63]. In this context it is important to find connections between ideal or axiomatically defined quantities and actual measurements. Therefore, attempts have been undertaken to propose experimental schemes how to measure time observables. For the measurement of the time of arrival a quantum optical model has been proposed in which the detection time of the first fluorescence photon of a two-level atom impinging on a laser illuminated region is taken as the arrival-time of the atom [64]. This first photon distribution can be linked to ideal quantities like Kijowski's distribution or the quantum mechanical flux [65].

For the experimental realization of such a quantum optical model, a major technical challenge is that it is necessary to achieve a laser profile which is sharp compared to the width of the wave packet in order have a well defined arrival point. At the same time, the atoms for which the arrival-time is to be observed have to be sufficiently slow so that quantum effects can be observed. These conditions are fulfilled by the present measurement scheme. The question arises whether one of the named ideal quantities is measured. As a major result of this part of the work, it will be shown that in fact none of the ideal arrival-time distributions is measured but rather a semi-classical distribution.

This part of the work is organized as follows: Chapter 4 gives background information on a few topics important for the following investigations. In Section 4.1 the experiment under investigation is described in more detail and relevant experimental data is given. In Section 4.2 the principle of the evanescent wave atomic mirror is explained and some theoretical background on the dipole force and quantum mechanical motion in exponential potentials is given. Section 4.3 contains a 
short review of the problem of quantum time of arrival. The theoretical concepts which are important in the context of the experiment in question are presented. In the following Chapter 5, the model for the description of the measurement procedure is presented and an expansion of the measured reflected norm in terms of the length of the time slit is given. Furthermore numerical calculations are presented which illustrate the different possible measuring set-ups. Finally a three-dimensional generalization of the model is presented.

In Appendix $\mathrm{C}$ a review of some basic properties of the Hilbert transform are given, together with the calculation of Hilbert transforms for two classes of functions needed in this work. 


\section{Chapter 4}

\section{Atoms in evanescent laser fields}

In this chapter some background information on the experiment is presented from which the basic idea of the detection scheme is taken which will be analyzed in this part of the work. First, in Section 4.1 a short description of the experiment itself is given. This includes information about the values of the physical parameters in the experiment which are of some importance for the model which will be developed later. Following this, Section 4.2 is concerned with the theoretical description of the evanescent wave atomic mirror. This is the principal component of the experiment and of the detection scheme to be developed. For one specific application of this scheme which will be discussed later a consideration based on classical mechanics suggests that it may be possible to measure a quantum arrival-time distribution. Section 4.3 therefore reviews certain aspects of this controversially discussed topic.

\subsection{Description of the experiment}

A schematic illustration of the experimental set-up is shown in Figure 4.1. A cloud of laser cooled Cesium atoms which has been cooled down to about $5 \mu \mathrm{K}$ is released from a magneto-optical trap and dropped onto an atomic mirror located $3 \mathrm{~mm}$ below the trap. This mirror consists of a prism illuminated from underneath by laser light as shown in Figure 4.2. The laser is totally reflected at the prism surface giving rise to an exponentially decreasing field above the surface. This so-called evanescent light field has a decay length on the sub-micrometer scale and forms the actual mirror. More precisely the decay length $\kappa^{-1}$ of the evanescent wave field is given by

$$
\kappa=\frac{2 \pi}{\lambda} \sqrt{n^{2} \sin ^{2} \theta-1},
$$

where $\lambda$ is the wave length of the laser, $n$ is the refractive index of the dielectric forming the prism, and $\theta$ is the angle of incidence of the laser beam. For the experiment the estimation $\kappa^{-1}=0.19 \mu \mathrm{m}$ was given [50].

The laser is blue detuned from the $6 S_{1 / 2} \leftrightarrow 6 P_{3 / 2}$ resonance line of the cesium atoms. This results in a repulsive force acting on the atoms which is called dipole 


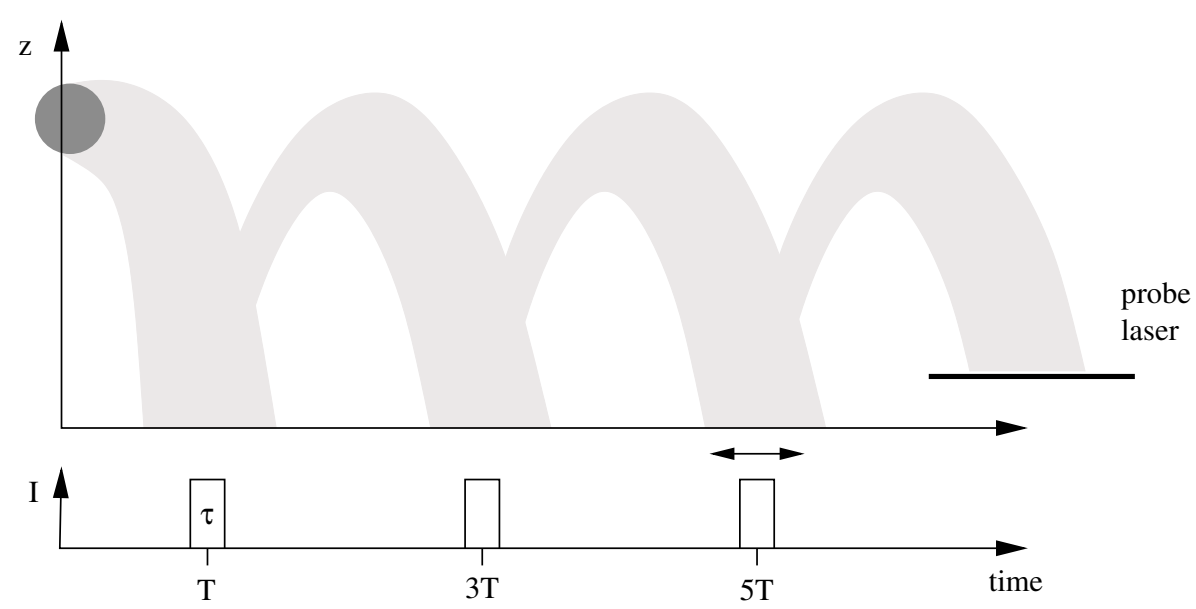

Figure 4.1: Sketch of the temporal diffraction experiment of Reference [49].

force [66]. By switching the laser field on and off the mirror can be switched on and off very quickly, in about $0.5 \mu \mathrm{s}$. With the laser switched off the atoms stick to the surface of the prism and are lost for the experiment. At the time $t=0$ when the atoms are released from the trap the mirror is in the off-state. It is turned on for a short time window, or pulse, of length $\tau$ centered at time $T=25 \mathrm{~ms}$ which corresponds to the classical falling time of the atoms $T=\sqrt{2 z_{0} / g}$, with $z_{0}$ the distance between trap and mirror and $g$ the gravitational acceleration. The mirror is switched on again at time $t=3 T$ later, again for a pulse of length $\tau$.

The resulting wave packet is measured by a third pulse which is shifted in time around $t=5 T$. This is done by determining the number of atoms that have been reflected by this pulse in a fluorescence experiment using probe laser. The integrated intensity of the fluorescence induced by this probe laser is proportional to the number of atoms that have been reflected. From a more quantum mechanical point of view the mirror cuts out a short section of the wave packet which impinges onto the mirror. The norm of this reflected part of the wave packet contains information about the value of the wave function at the position of the mirror. The shorter the duration of this measurement pulse is the more accurate this information will be. These simple considerations show that it might be interesting to study this detection process in more detail which will be the aim of the following chapters of this work. Since the atomic gas is very dilute the atoms do not interact with each other and the experiment can be seen as an experiment with single atoms.

For sufficiently small values of $\tau$ the wave packet describing the atom after the second pulse exhibits sidebands due to temporal diffraction effects. In the experiments this was seen for approximately $\tau \leq 35 \mu \mathrm{s}$. The concept of diffraction in time in quantum mechanics goes back to Moshinsky, who considered the question of what happens to a monochromatic beam of particles interrupted by a shutter if this shutter is suddenly removed $[67,68]$. This concept was then further investi- 


\begin{tabular}{|l|c|l|}
\hline physical parameter & Notation & exp. value \\
\hline \hline wavelength of the atomic transition & $\lambda$ & $852 \mathrm{~nm}$ \\
\hline decay rate of the transition & $\Gamma$ & $2 \pi \times 5.3 \mathrm{MHz}$ \\
\hline saturation intensity & $I_{\text {sat }}$ & $2.2 \mathrm{~mW} / \mathrm{cm}^{2}$ \\
\hline laser intensity & $I$ & $\approx 35 \mathrm{~W} / \mathrm{cm}^{2}$ \\
\hline Rabi frequency & $\Omega_{0}=\sqrt{\Gamma^{2} I_{\text {sat }} / I}$ & $\approx 4.2 \mathrm{~s}^{-1}$ \\
\hline laser detuning & $\Delta$ & $9.5 \mathrm{GHz}$ \\
\hline inverse decay length of the laser field & $\kappa$ & $5.3 \times 10^{6} \mathrm{~m}^{-1}$ \\
\hline cesium mass & $m_{\mathrm{Cs}}$ & $2.2 \times 10^{-25} \mathrm{~kg}$ \\
\hline mean velocity of the atoms & $v_{0}$ & $0.25 \mathrm{~m} / \mathrm{s}^{2}$ \\
\hline mean wave number of the atoms & $k_{0}=m_{\mathrm{Cs}} v_{0} / \hbar$ & $5.3 \times 10^{8} \mathrm{~m}^{-1}$ \\
\hline kinetic energy in units of $\hbar$ & $E_{\mathrm{kin}} / \hbar$ & $65.3 \times 10^{6} \mathrm{~s}^{-1}$ \\
\hline
\end{tabular}

Table 4.1: A compilation of the experimental data relevant for the investigations in this work. The Rabi frequency given here is an estimation based on the intensity of the laser outside the prism and on experimental values given in reference [50]. It should therefore merely be seen as an upper boundary.

gated theoretically and generalized by several authors [69, 70, 71]. The experiment of Reference [49] is a first realization of this concept for atoms.

For practical purposes connected with the detection scheme which will be developed in the following it is an important point that the wave packets produced by the first two pulses are broad compared to the decay length of the mirror. This allows to resolve the wave packet by a measurement with the mirror. For example, for $\tau=30 \mu$ s the measured distribution had a full width at half maximum (FWHM) of about $\Delta t=80 \mu \mathrm{s}$. This corresponds, via $\Delta x=v_{0} \Delta t$, to $\Delta x=20 \mu \mathrm{m}$. Here, $v_{0}$ is the classical velocity at the position of the mirror for atoms initially at rest.

The mechanism for the formation of the wave packets will now be shortly illustrated in a simple classical picture. The first two pulses select atoms with a certain range of velocities. Let the surface of the mirror be positioned at $z=0$. The classical trajectories of the atoms between the first and second rebound are given by

$$
z(t)=v_{0} t-\frac{g}{2} t^{2}
$$

where now $t=0$ is the time at which the atoms leave the mirror surface after the first reflection. In order to be reflected, the atoms have to reach the mirror again 
after a time $2 T-\tau \leq t \leq 2 T+\tau$. This yields

$$
g\left(T-\frac{\tau}{2}\right) \leq v_{0} \leq g\left(T+\frac{\tau}{2}\right)
$$

as the range of possible velocities just before the first reflection and thus just after the second reflection. Assuming a broad, practically uniformly distributed, initial velocity distribution, one arrives at a triangular velocity distribution centered around $v_{0}=g T$ with a FWHM of $\Delta v=g \tau / 2$ just after the second rebound. At the same time the spatial distribution of the atomic cloud is limited by $\Delta x=v_{0} \tau$. For $\tau=30 \mu$ s one has $\Delta v=0.15 \mathrm{~mm} / \mathrm{s}$ and $\Delta x=7.5 \mu \mathrm{m}$. Although the velocity distribution is quite sharp there is still some spreading in the spatial distribution until the atoms reach the measurement pulse. Atoms with velocity $v=g T$ reach the mirror again after a time $t=2 T$ whereas atoms with velocity $v \pm \Delta v$ arrive after a time $t=2 T \mp \tau$. Thus the atoms arrive at the mirror for the third rebound with a width of roughly $\Delta x=3 v_{0} \tau=22.5 \mu \mathrm{m}$ which is in reasonably good agreement with the experimental result. In the following the preparation process itself disregarded. It is simply assumed that a preparation of a wave packet with the above properties is possible.

\subsection{Evanescent wave atomic mirrors}

The main component of the experiment of Reference [49] is an atomic mirror based on an evanescent light field on the surface of a dielectric prism. It is used in the following as a measuring device to extract information from the wave packet. This chapter gives some theoretical background on the basic mechanism of such a mirror.

The original idea of using an evanescent wave light field as a mirror for atoms is due to Cook and Hill [72]. The first experimental realization of such a mirror was achieved by Balykin et al. [73, 74] for a beam of thermal atoms at grazing incidence and by Kasevitch et al. [75] for the so-called "trampoline" configuration in which laser cooled atoms are released from an atomic trap and being dropped onto the mirror in perpendicular incidence.

The basic principle of the evanescent wave mirror is this. If an electromagnetic wave propagating in a dielectric undergoes total reflection at the surface of the material then on the outside of the medium there is an evanescent wave, i.e. a field with an amplitude which is exponentially decreasing with the distance to the surface [76]. The decay length $\kappa^{-1}$ as given by Equation (4.1) in the previous section is typically of the order of a wave length and $\kappa^{-1}=0.19 \mu \mathrm{m}$ in the example given above. Due to this intensity gradient, an atom which is placed into the evanescent field experiences a force, the so-called dipole force. If the frequency of the light field is tuned above an atomic resonant frequency this force is repulsive and a sufficiently slow atom is reflected without reaching the surface. A sketch of the basic design of such a mirror is given in Figure 4.2. 


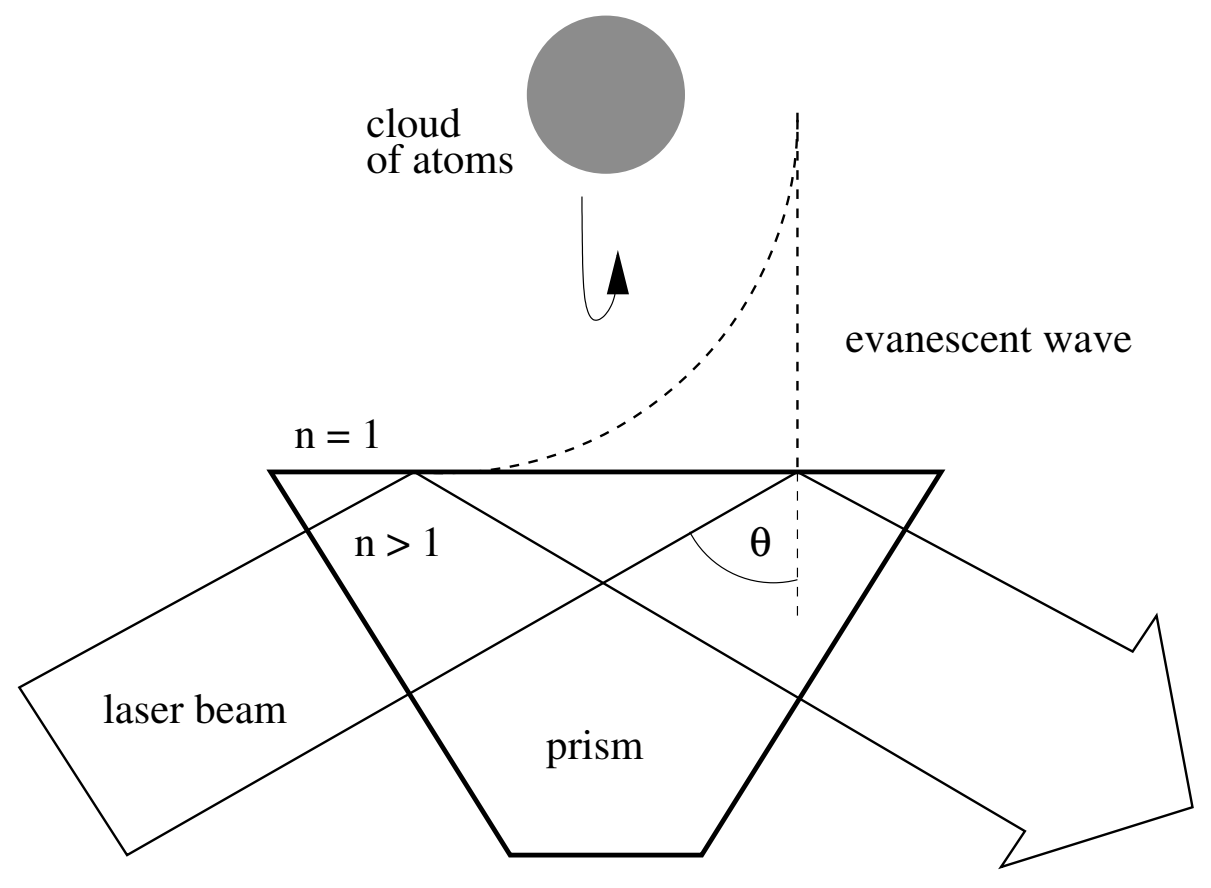

Figure 4.2: Sketch of the principle of an evanescent wave mirror.

In order to set forth this mechanism in more detail the following two sections deal with the dipole force and the quantum mechanical motion of atoms in exponential potentials.

\subsubsection{The dipole force}

Usually the derivation of the dipole force is done via a semi-classical approach using Bloch equations [66]. In this approach the atom is considered to be point-like and the internal states of the atom are assumed to follow the laser field applied at any given point adiabatically. However, the assumption of the atom to be point-like is not fulfilled in the set-up to be discussed here. In contrary, a very broad wave packet is being prepared. Therefore a different approach is chosen here which is based on the partitioning technique due to Zwanzig and Mori [77, 78, 79].

The calculation starts with the conditional Hamiltonian for a moving atom with internal two-level structure interacting with a laser field

$$
H_{\mathrm{c}}=\frac{\hat{p}^{2}}{2 m}-\hbar(\Delta+\mathrm{i} \gamma / 2)|2\rangle\langle 2|+\frac{\hbar \Omega(\hat{x})}{2}(|1\rangle\langle 2|+| 2\rangle\langle 1|)
$$

describing the time evolution until the emission of the first photon in the framework of the quantum jump approach [80]. Here, $\gamma$ is the decay rate of the excited level and $\Delta$ is the detuning of the laser. For the present problem of an evanescent wave mirror the position dependence of the Rabi frequency is given by

$$
\Omega(\hat{x})=\Omega_{0} \mathrm{e}^{\kappa \hat{x}}
$$


although the following calculation is valid for more general laser profiles. In contrast to the experiment the atom is now assumed to be moving horizontally and coming in from the far left. Gravitation is discarded in the following. The prism surface is assumed to be at $x=0$. Therefore, only the region $x \leq 0$ is of interest. The initial state of the system is given by

$$
\boldsymbol{\psi}_{0}=\psi_{0}|1\rangle
$$

where $\psi_{0}$ is the initial wave function for the center of mass motion. Using the projectors on the ground and excited states

$$
P=|1\rangle\langle 1|, \quad Q=| 2\rangle\langle 2|, \quad Q+P=\mathbb{1}
$$

the Schrödinger equation for the conditional time development can be written as

$$
\dot{\boldsymbol{\psi}}_{t}=-\frac{\mathrm{i}}{\hbar} H_{\mathrm{c}} \boldsymbol{\psi}_{t}=-\frac{\mathrm{i}}{\hbar}(P+Q) H_{\mathrm{c}}(P+Q) \boldsymbol{\psi}_{t}
$$

The projections of this equation onto the ground state and the excited state are given by

$$
P \dot{\boldsymbol{\psi}}_{t}=-\frac{\mathrm{i}}{\hbar} P H_{\mathrm{c}} P P \boldsymbol{\psi}_{t}-\frac{\mathrm{i}}{\hbar} P H_{\mathrm{c}} Q Q \boldsymbol{\psi}_{t}
$$

and

$$
Q \dot{\boldsymbol{\psi}}_{t}=-\frac{\mathrm{i}}{\hbar} Q H_{\mathrm{c}} Q Q \boldsymbol{\psi}_{t}-\frac{\mathrm{i}}{\hbar} Q H_{\mathrm{c}} P P \boldsymbol{\psi}_{t}
$$

respectively. Defining $f(t)=-\frac{\mathrm{i}}{\hbar} Q H_{\mathrm{c}} P P \boldsymbol{\psi}_{t}$ the formal solution of Equation (4.10) is given by

$$
\begin{aligned}
Q \boldsymbol{\psi}_{t} & =\mathrm{e}^{-\frac{\mathrm{i}}{\hbar} Q H_{\mathrm{c}} Q t} Q \boldsymbol{\psi}_{0}+\int_{0}^{t} \mathrm{~d} s \mathrm{e}^{-\frac{\mathrm{i}}{\hbar} Q H_{\mathrm{c}} Q(t-s)} f(s) \\
& =-\frac{\mathrm{i}}{\hbar} \int_{0}^{t} \mathrm{~d} s \mathrm{e}^{-\frac{\mathrm{i}}{\hbar} Q H_{\mathrm{c}} Q(t-s)} Q H_{\mathrm{c}} P P \boldsymbol{\psi}_{s},
\end{aligned}
$$

since $Q \boldsymbol{\psi}_{0}=0$. Inserting this into equation (4.9) gives

$$
P \dot{\boldsymbol{\psi}}_{t}=-\frac{\mathrm{i}}{\hbar} P H_{\mathrm{c}} P P \boldsymbol{\psi}_{t}-\frac{1}{\hbar^{2}} P H_{\mathrm{c}} Q \int_{0}^{t} \mathrm{~d} s \mathrm{e}^{-\frac{\mathrm{i}}{\hbar} Q H_{\mathrm{c}} Q(t-s)} Q H_{\mathrm{c}} P P \boldsymbol{\psi}_{s}
$$

as an integro-differential equation for the time development of the state $P \boldsymbol{\psi}_{t}$. Using the explicit expressions

$$
P H_{\mathrm{c}} P=\frac{\hat{p}^{2}}{2 m}, \quad Q H_{\mathrm{c}} Q=\frac{\hat{p}^{2}}{2 m}-\hbar(\Delta+\mathrm{i} \gamma / 2), \quad P H_{\mathrm{c}} Q=Q H_{\mathrm{c}} P=\frac{\hbar \Omega(\hat{x})}{2}
$$

and applying the substitution $\tau=t-s$ yields

$$
P \dot{\boldsymbol{\psi}}_{t}=-\frac{\mathrm{i}}{\hbar} \frac{\hat{p}^{2}}{2 m} P \boldsymbol{\psi}_{t}-\frac{\Omega(\hat{x})}{4} \int_{0}^{t} \mathrm{~d} \tau \mathrm{e}^{-\frac{\mathrm{i}}{\hbar}\left(\frac{\hat{p}^{2}}{2 m}-\hbar(\Delta+\mathrm{i} \gamma / 2)\right) \tau} \Omega(\hat{x}) P \boldsymbol{\psi}_{t-\tau}
$$


In order to simplify this expression it is possible to apply a kind of Markov approximation to the integrand. If $|\hbar(\Delta+\mathrm{i} \gamma / 2)| \gg\left|\frac{\hat{p}^{2}}{2 m}\right|$ the integrand is fastly oscillating (if $\Delta \gg \gamma$ ) or rapidly damped so that only the contribution from $\tau=0$ plays a significant role in the integral. Therefore, to good approximation, one can put $\tau=0$ in $P \boldsymbol{\psi}_{t-\tau}$ and the upper integration limit can be extended to infinity. Then one has

$$
\begin{aligned}
P \dot{\boldsymbol{\psi}}_{t} & =-\frac{\mathrm{i}}{\hbar} \frac{\hat{p}^{2}}{2 m} P \boldsymbol{\psi}_{t}-\frac{\Omega(\hat{x})}{4} \int_{0}^{\infty} \mathrm{d} \tau \mathrm{e}^{-\frac{\mathrm{i}}{\hbar}\left(\frac{\hat{p}^{2}}{2 m}-\hbar(\Delta+\mathrm{i} \gamma / 2)\right) \tau} \Omega(\hat{x}) P \boldsymbol{\psi}_{t} \\
& =-\frac{\mathrm{i}}{\hbar} \frac{\hat{p}^{2}}{2 m} P \boldsymbol{\psi}_{t}-\frac{\hbar \Omega(\hat{x})}{4} \frac{1}{\mathrm{i}\left(\frac{\hat{p}^{2}}{2 m}-\hbar(\Delta+\mathrm{i} \gamma / 2)\right)} \Omega(\hat{x}) P \boldsymbol{\psi}_{t} .
\end{aligned}
$$

When the kinetic energy term in the integrand is neglected the Rabi frequency commutates with the integral yielding

$P \dot{\boldsymbol{\psi}}_{t}=-\frac{\mathrm{i}}{\hbar} \frac{\hat{p}^{2}}{2 m} P \boldsymbol{\psi}_{t}+\frac{\Omega^{2}(\hat{x})}{4 \mathrm{i}(\Delta+\mathrm{i} \gamma / 2)} P \boldsymbol{\psi}_{t}=-\frac{\mathrm{i}}{\hbar} \frac{\hat{p}^{2}}{2 m} P \boldsymbol{\psi}_{t}-\mathrm{i} \frac{\Omega^{2}(\hat{x})}{4\left(\Delta^{2}+\gamma^{2} / 4\right)}(\Delta-\mathrm{i} \gamma / 2) P \boldsymbol{\psi}_{t}$.

If, in addition, the relation $\Delta \gg \gamma$ is fulfilled one finally gets

$$
P \dot{\boldsymbol{\psi}}_{t}=-\frac{\mathrm{i}}{\hbar}\left[\frac{\hat{p}^{2}}{2 m}+\frac{\hbar \Omega^{2}(\hat{x})}{4 \Delta}\right] P \boldsymbol{\psi}_{t} .
$$

as an effective Schrödinger equation for the ground state. The second term on the right hand side can be regarded as a potential, namely the potential of the dipole force. It is identical to the term obtained by the usual derivation. In the case investigated here, it is given by

$$
V(\hat{x})=\frac{\hbar \Omega^{2}(\hat{x})}{4 \Delta}=\frac{\hbar \Omega_{0}^{2}}{4 \Delta} \mathrm{e}^{2 \kappa \hat{x}} .
$$

Note that in contrast to the first part of the work only the translational degrees of freedom have to be considered whereas the internal degrees of freedom can be disregarded.

The approximation of Equation (4.16) is only valid for $\Omega \ll \Delta$ since else the state $P \boldsymbol{\psi}_{t-\tau}$ can be fastly oscillating itself. This can be seen in the simplified case, when $\Omega$ is a number instead of an operator. This is the case, for example, when $\Omega$ is constant over the whole real axis. Then Equation (4.15) can be solved by applying a Laplace transform. The solution is given by

$$
P \boldsymbol{\psi}_{t}=\frac{\Omega_{\mathrm{eff}}-\Delta_{\gamma}}{2 \Omega_{\mathrm{eff}}} \mathrm{e}^{-\frac{\mathrm{i}}{\hbar}\left(\frac{\hat{p}^{2}}{2 m}-\frac{\hbar}{2}\left(\Delta_{\gamma}+\Omega_{\mathrm{eff}}\right)\right) t}+\frac{\Omega_{\mathrm{eff}}+\Delta_{\gamma}}{2 \Omega_{\mathrm{eff}}} \mathrm{e}^{-\frac{\mathrm{i}}{\hbar}\left(\frac{\hat{p}^{2}}{2 m}-\frac{\hbar}{2}\left(\Delta_{\gamma}-\Omega_{\mathrm{eff}}\right)\right) t},
$$

with $\Delta_{\gamma}=\Delta+\mathrm{i} \gamma / 2$ and $\Omega_{\text {eff }}=\sqrt{\Omega^{2}+\Delta_{\gamma}^{2}}$. The first term of this solution oscillates with a frequency larger than $2 \Delta$ even if $\Omega \ll \Delta$. If the condition $\Omega \ll \Delta$ (and also $\Delta \gg \gamma$ as above) is fulfilled the solution (4.21) can be approximated by

$$
P \boldsymbol{\psi}_{t}=\frac{\Omega^{2}}{4 \Delta^{2}} \mathrm{e}^{-\frac{\mathrm{i}}{\hbar}\left(\frac{\hat{p}^{2}}{2 m}-\hbar \Delta\right) t}+\mathrm{e}^{-\frac{\mathrm{i}}{\hbar}\left(\frac{\hat{p}^{2}}{2 m}-\frac{\hbar \Omega^{2}}{4 \Delta}\right) t}
$$


so the fastly oscillating term can be neglected.

The experimental values given in Table 4.1 do not strictly fulfill the condition $\Omega \ll \Delta$ since $\Delta / \Omega \approx 2$. The kinetic energy of the atoms in the experiment, however is much smaller than the maximal value of the potential given by Equation (4.20). Thus, the atoms will be already reflected at a position at which $\Delta / \Omega \gg 1$ indeed is fulfilled. In addition one can show that the mean number of photons to be emitted by an atom during its flight through the evanescent wave field is smaller than one for the given parameter values. This is another indication for the validity of the approximation applied in the calculation of the optical potential.

\subsubsection{Atoms in exponential potentials}

In this section the behavior of a quantum particle in an exponential potential is discussed in order to illustrate the simplifications applied to the model for the detection scheme given further below. The argument mainly follows Reference [81].

The Hamiltonian of the problem is given by

$$
H=\frac{\hat{p}^{2}}{2 m}+\frac{\hbar^{2} k_{\max }^{2}}{2 m} \mathrm{e}^{2 \kappa \hat{x}}
$$

By defining $k_{\max }=\sqrt{\frac{m \Omega_{0}^{2}}{2 \hbar \Delta}}$ one obtains the optical potential of the previous section. In order to describe the atomic reflection from this exponential potential the stationary Schrödinger equation

$$
\left[-\frac{\mathrm{d}^{2}}{\mathrm{~d} x^{2}}+k_{\max }^{2} \mathrm{e}^{2 \kappa x}\right] \psi(x)=k^{2} \psi(x)
$$

will now be solved exactly. This done by applying the transformation

$$
x \rightarrow u(x)=\alpha \mathrm{e}^{\kappa x},
$$

with $\alpha=k / \kappa$. Equation (4.24) then takes the form

$$
\left[u^{2} \frac{\mathrm{d}^{2}}{\mathrm{~d} u^{2}}+u \frac{\mathrm{d}}{\mathrm{d} u}-\left(u^{2}-\alpha^{2}\right)\right] \psi(u)=0 .
$$

The solution to this equation is given by linear combinations of the modified Bessel functions $I_{ \pm \mathrm{i} \alpha}(u)$ defined by [82]

$$
I_{ \pm \mathrm{i} \alpha}(u)=\left(\frac{u}{2}\right)^{ \pm \mathrm{i} \alpha} \sum_{k=0}^{\infty} \frac{\left(\frac{u}{2}\right)^{2 k}}{k ! \Gamma(k+1 \pm \mathrm{i} \alpha)},
$$

where $\Gamma(z)$ is the Euler gamma function. In order to find a unique solution one has to consider the behavior of this function for $x \rightarrow \pm \infty$ which is equivalent to $u \rightarrow \infty$ and $u \rightarrow 0$, respectively. The former limit can be computed by using an asymptotic 
expansion [82] and the latter limit by using the first term of the expansion (4.27). This gives

$$
\begin{aligned}
x \rightarrow-\infty: & I_{ \pm \mathrm{i} \alpha}(u(x)) & \simeq \sqrt{\frac{\sinh (\pi \alpha)}{\pi \alpha}} \mathrm{e}^{ \pm[\alpha(\kappa x+\ln \beta / 2)-\arg \Gamma(1+\mathrm{i} \alpha)]} \\
u \rightarrow+\infty: & I_{ \pm \mathrm{i} \alpha}(u) & \sim \frac{1}{\sqrt{2 \pi u}} \mathrm{e}^{u}(1+\mathcal{O}(1 / u)),
\end{aligned}
$$

with $\beta=k_{\max } / \kappa$. Since physically reasonable solutions have to vanish for $x \rightarrow+\infty$ the eigenstate with wave number $k$ is given by

$$
\phi_{k}=\frac{1}{2 \mathrm{i}} \sqrt{\frac{\pi \alpha}{\sinh \pi \alpha}}\left[I_{\mathrm{i} \alpha}(u(x))-I_{-\mathrm{i} \alpha}(u(x))\right]=-\sqrt{\frac{\alpha}{\pi} \sinh (\pi \alpha)} K_{\mathrm{i} \alpha}(u(x)),
$$

with the modified Bessel function of the second kind $K_{\mathrm{i} \alpha}(z)$. It has the asymptotic expansion

$$
u \rightarrow \infty: K_{\mathrm{i} \alpha}(u) \sim \sqrt{\frac{\pi}{2 u}} \mathrm{e}^{-u}(1+\mathcal{O}(1 / u))
$$

Thus, the eigenstates of Equation (4.30) are exponentially damped for $x \rightarrow \infty$ and equation (4.28) shows that it asymptotically describes a plane wave with initial momentum $\hbar k$ coming in from the far left which is totally reflected and is experiencing a phase shift

$$
\Delta \phi(k)=2 \alpha \ln (\beta / 2)-2 \arg \Gamma(1+\mathrm{i} \alpha)
$$

in the reflection process. If one considers an atomic wave packet

$$
\psi(x, t)=\int_{0}^{\infty} \mathrm{d} k \widetilde{\psi}(k) \phi_{k} \mathrm{e}^{-\mathrm{i} \frac{\hbar k^{2}}{2 m} t}
$$

with a momentum amplitude $\widetilde{\psi}(k)$ peaked around some average wave number $k_{0}$ one can use the method of stationary phase to calculate the position $x_{\mathrm{wp}}(t)$ of the center of the wave packet. For the asymptotic incoming and outgoing state on finds

$$
\begin{aligned}
& x_{\mathrm{wp}}^{\text {in }}(t)=\frac{\hbar k_{0}}{m} t-\frac{1}{2}\left(\frac{\partial \Delta \phi(k)}{\partial k}\right)_{k_{0}} \\
& x_{\mathrm{wp}}^{\text {out }}(t)=-\frac{\hbar k_{0}}{m} t-\frac{1}{2}\left(\frac{\partial \Delta \phi(k)}{\partial k}\right)_{k_{0}} .
\end{aligned}
$$

Thus, as far as the asymptotic behavior is concerned the exponential potential behaves as an infinitely steep step potential located at the position

$$
\xi_{0}=-\frac{1}{2}\left(\frac{\partial \Delta \phi(k)}{\partial k}\right)_{k_{0}}=-\frac{1}{\kappa}(\ln (\beta / 2)-\operatorname{Re} \Psi(1+\mathrm{i} \bar{\alpha})),
$$


where $\Psi(z)$ is the digamma function defined by $\Psi(z)=\partial \ln \Gamma(z) / \partial z$ and $\bar{\alpha}=k_{0} / \kappa$. For $\bar{\alpha} \gg 1$, as is the case in the experiment in question, this position simplifies to

$$
\xi_{0}=-\frac{1}{\kappa} \ln \left(\frac{\beta}{2 \bar{\alpha}}\right) .
$$

Of course, the actual optical potential is not growing to infinity for $x \rightarrow \infty$ but reaches a maximum value $\hbar \Omega_{0}^{2} / 4 \Delta$ at the surface of the prism. Therefore it would be more realistic to use a potential which is cut at $x=0$. This is described by the Hamiltonian

$$
H=\frac{\hat{p}^{2}}{2 m}+\frac{\hbar^{2} k_{\max }^{2}}{2 m} \mathrm{e}^{2 \kappa x} \theta(-x)
$$

with the Heaviside step function $\theta(x)$. The eigenfunctions for an atom incoming from the left can be written in the form

$$
\begin{array}{ll}
x<0: & \phi(x)=\sqrt{\frac{\pi \alpha}{\sinh (\pi \alpha)}}\left[I_{\mathrm{i} \alpha}(u(x))+A I_{-\mathrm{i} \alpha}(u(x))\right] \\
x \geq 0: & \phi(x)=B \mathrm{e}^{\mathrm{i} k_{0} x}=B \mathrm{e}^{\mathrm{i} \alpha \kappa x} .
\end{array}
$$

From the matching conditions at $x=0$ one then obtains the prefactors

$$
A=-\frac{I_{\mathrm{i} \alpha+1}(\beta)}{I_{-(\mathrm{i} \alpha+1)}(\beta)} \quad B=-2 \mathrm{i} \sqrt{\frac{\alpha}{\beta}} \sqrt{\frac{\sinh (\pi \alpha)}{\pi \beta}} \frac{1}{I_{-\mathrm{i} \alpha-1}(\beta)},
$$

which obey the relation $A^{2}+B^{2}=1$, as they should. This can be shown using properties of the Bessel functions [82]. Numerical analysis using the experimental values shows that for $\beta / \alpha=1.05$ the transmission probability is already smaller than $|B|^{2}=0.5 \%$. For the typical kinetic energies of the experiment the optical potential can be regarded as completely reflecting.

\subsection{Arrival-times in quantum mechanics}

In the analysis of the detection scheme motivated by the experiment presented in Section 4.1 the question arises, whether the distribution obtained in a particular set-up can be interpreted as an arrival-time distribution. In order to provide the necessary background, in this section a few important concepts and definitions of arrival-times are presented. Since quantum arrival-times are not the main issue of this work only a short overview is given. More detailed discussions can be found in $[63,83]$.

\subsubsection{Classical arrival-time}

Before discussing the quantum case it is useful to review the main properties of classical arrival-times. In the following only the straightforward situation of arrivaltimes of free particles will be treated. For the discussion of the case of interacting 
particles, where topics like multiple crossings have to be considered, see Reference [63].

In classical mechanics the arrival-time at $x=x_{\mathrm{A}}$ of a freely moving particle with initial position $x_{0}<x_{\mathrm{A}}$ and initial momentum $p_{0}>0$ is given by

$$
t_{\mathrm{A}}=\frac{m\left(x_{\mathrm{A}}-x_{0}\right)}{p_{0}} .
$$

Since the particle crosses the point $x_{0}$ once and only once, no distinction has to be made between arrival-time and first arrival-time. For an ensemble of classical particles the distribution of arrival-times is given by the probability flux or current density $J^{\mathrm{cl}}\left(t, x_{\mathrm{A}}\right)$. It can be written as the average of the phase-space function $\mathscr{J}\left(x, p ; x_{\mathrm{A}}\right)=\frac{p}{m} \delta\left(x-x_{\mathrm{A}}\right)$ evaluated with the phase space distribution function $\rho_{t}(x, p)$

$$
J^{\mathrm{cl}}\left(t, x_{\mathrm{A}}\right)=\int_{-\infty}^{\infty} \mathrm{d} x \int_{0}^{\infty} \mathrm{d} p \rho_{t}(x, p) \frac{p}{m} \delta\left(x-x_{\mathrm{A}}\right)
$$

where $\rho_{t}(x, p \leq 0)=0$. This is the classical probability flux or current density. The average arrival-time is given by the first moment of $J^{\text {cl }}$

$$
\left\langle t_{\mathrm{A}}\right\rangle=\int_{-\infty}^{\infty} t J^{\mathrm{cl}}\left(t, x_{\mathrm{A}}\right)
$$

By use of the trajectory equation $x=x_{0}+\frac{p_{0}}{m} t$ and Liouville's theorem this can be rewritten as the average of the times $t_{\mathrm{A}}$ with respect to the phase-space distribution function

$$
\left\langle t_{\mathrm{A}}\right\rangle=\int_{-\infty}^{\infty} \mathrm{d} x_{0} \int_{0}^{\infty} \mathrm{d} p_{0} \rho_{0}\left(x_{0}, p_{0}\right) \frac{m\left(x_{\mathrm{A}}-x_{0}\right)}{p_{0}} .
$$

For the more general case of arbitrary initial momentum the flux $J^{\mathrm{cl}}\left(t, x_{\mathrm{A}}\right)$ itself is not the arrival-time distribution anymore. However, the positive flux

$$
J_{+}^{\mathrm{cl}}\left(t, x_{\mathrm{A}}\right)=\int_{-\infty}^{\infty} \mathrm{d} x \int_{0}^{\infty} \mathrm{d} p \rho_{t}(x, p) \frac{p}{m} \delta\left(x-x_{\mathrm{A}}\right)
$$

and minus the negative flux

$$
J_{-}^{\mathrm{cl}}\left(t, x_{\mathrm{A}}\right)=\int_{-\infty}^{\infty} \mathrm{d} x \int_{-\infty}^{0} \mathrm{~d} p \rho_{t}(x, p) \frac{p}{m} \delta\left(x-x_{\mathrm{A}}\right)
$$

are unnormalized arrival-time distributions for particles moving rightwards and leftwards, respectively. As a consequence the sum of these two expressions

$$
J_{\text {tot }}^{\mathrm{cl}}\left(t, x_{\mathrm{A}}\right)=J_{+}^{\mathrm{cl}}\left(t, x_{\mathrm{A}}\right)-J_{-}^{\mathrm{cl}}\left(t, x_{\mathrm{A}}\right)=\int_{-\infty}^{\infty} \mathrm{d} x \int_{-\infty}^{\infty} \mathrm{d} p \rho_{t}(x, p) \frac{|p|}{m} \delta\left(x-x_{\mathrm{A}}\right)
$$

gives the total arrival-time distribution in this case. 


\subsubsection{Quantum arrival-times}

The question of how to incorporate the time of arrival as an observable into standard quantum mechanics is a controversially discussed topic with a long history. In principal, one has to distinguish clearly between attempts of defining arrival-time operators and approaches which deal with the proposal of arrival-time distributions and mean arrival-times. In this section only the latter approach will be discussed.

\section{Quantum mechanical flux}

Since for particles with only positive momenta the classical arrival-time distribution is given by the current density $J^{\mathrm{cl}}\left(t, x_{\mathrm{A}}\right)$ it seems obvious to use the analogous quantity, namely the quantum mechanical flux

$$
J\left(x_{\mathrm{A}}, t\right)=\frac{\hbar}{m} \operatorname{Im}\left(\overline{\psi\left(x_{\mathrm{A}}, t\right)} \psi^{\prime}\left(x_{\mathrm{A}}, t\right)\right)
$$

as the arrival-time distribution for the quantum mechanical state $\psi(x, t)$ at the arrival point $x_{\mathrm{A}}$. However, this quantity is not necessarily positive. For wave packets with purely positive wave number contributions $J(x, t)$ can become negative on arbitrarily long, but finite, time intervals [84]. This is the so-called back-flow effect [85]. In many cases, nevertheless, the flux provides a well-defined expression for an arrival-time distribution.

The situation becomes more difficult when one tries to carry over the general classical result (4.47) to quantum mechanics in the same way. The quantum mechanical flux can be seen as a quantization of the phase-space function $\mathscr{J}=\frac{p}{m} \delta\left(x-x_{\mathrm{A}}\right)$. In the general case one analogously has to consider the corresponding functions $\mathscr{J}_{+}$and $\mathscr{J}_{-}$. The problem is here that for example the quantization of $\mathscr{J}_{+}=\frac{p}{m} \delta\left(x-x_{\mathrm{A}}\right) \theta(p)$ is non-unique because the flux operator does not commute with $\theta(\hat{p})$. The same applies for $\mathscr{J}_{-}$and there are infinitely many symmetrization possibilities [63].

\section{Kijowski's distribution}

A different quantum arrival-time distribution based on classical correspondence has been given by Kijowski $[86,87]$. He defined a number of axioms which in the classical case lead to the right distribution, namely that of Equation (4.42). Carried over to the quantum case these axioms lead to a uniquely defined distribution. For free particles with only positive momentum components this distribution is given by

$$
\begin{aligned}
\Pi_{\mathrm{K}}\left(t, x_{\mathrm{A}}\right) & =\left\langle\psi_{t}\left|\frac{1}{m} \hat{p}^{1 / 2} \delta\left(\hat{x}-x_{\mathrm{A}}\right) \hat{p}^{1 / 2}\right| \psi_{t}\right\rangle \\
& =\frac{\hbar}{2 \pi m}\left|\int_{0}^{\infty} \mathrm{d} k \widetilde{\psi}(k) \sqrt{k} \mathrm{e}^{-\mathrm{i} \frac{\hbar k^{2}}{2 m} t} \mathrm{e}^{\mathrm{i} k x_{\mathrm{A}}}\right|^{2}
\end{aligned}
$$

for the arrival at $x=x_{\mathrm{A}}$. This distribution is called Kijowski's distribution. Note that the operator in Equation (4.49) can be seen as a positive quantization of the 
classical expression (4.42). For positive momentum components $\Pi_{K}$ is identical to the distribution later obtained [88] by means of positive operator valued measures (POVMs) from the so-called Aharonov-Bohm arrival-time operator [89].

For interacting particles and also for wave packets with arbitrary momentum components the axiomatic approach of Kijowski does not seem to be directly applicable. Generalizations for particles in external potentials are still subject of research $[90,91]$.

Recently a quantum optical scheme for measuring arrival-times has been proposed [64]. It could be shown that the arrival-time distribution resulting from this operational scheme can be linked to the quantum mechanical flux as well as to Kijowski's distribution in certain limits of the parameters involved [64, 65]. This approach also allows for the generalization of Kijowski's distribution to the case of particles with arbitrary momentum components and to the case of particles in an external potential [92].

\section{Expansion of Kijowski's distribution}

As mentioned in the last section Kijowski's distribution (4.49) can be written in the form

$$
\Pi_{\mathrm{K}}\left(t, x_{\mathrm{A}}\right)=\left\langle\psi_{t}\left|\frac{1}{m} \hat{p}^{1 / 2} \delta\left(\hat{x}-x_{\mathrm{A}}\right) \hat{p}^{1 / 2}\right| \psi_{t}\right\rangle .
$$

For wave packets which have a sharply peaked momentum distribution around some momentum $p_{0}$ the operator $\hat{p}^{1 / 2}$ can be expanded in terms of powers of $\left(\hat{p}-p_{0}\right)$ [93],

$$
\hat{p}^{1 / 2}=p_{0}^{1 / 2}+\frac{1}{2} p_{0}^{-1 / 2}\left(\hat{p}-p_{0}\right)+\mathcal{O}\left(\left(\hat{p}-p_{0}\right)^{2}\right) .
$$

In the following $p_{0}$ is taken to be the first moment $p_{0} / \hbar=\left.\int \mathrm{d} k k \widetilde{\psi(k)}\right|^{2}$ of the momentum distribution of the wave packet. Then, inserting the expansion into Equation (4.50) gives to zeroth order the particle's density at the arrival point $x_{\mathrm{A}}$ times the mean velocity

$$
\Pi_{\mathrm{K}}\left(t, x_{\mathrm{A}}\right)=v_{0}\left|\psi\left(x_{\mathrm{A}}, t\right)\right|^{2}+\mathcal{O}\left(\left(\hat{p}-p_{0}\right)\right) .
$$

this is also a possible time of arrival distribution which has been studied for example in Reference [94] which can be seen as a semi-classical distribution since it can be obtained from the classical term by replacing the classical spatial distribution function by the quantum mechanical probability density. More accurately the normalized distribution should be written as

$$
\Pi_{\mathrm{sc}}\left(t, x_{\mathrm{A}}\right)=\left|\psi\left(x_{\mathrm{A}}, t\right)\right|^{2} / \int \mathrm{d} t\left|\psi\left(x_{\mathrm{A}}, t\right)\right|^{2}
$$

because for wave packets with only positive momenta one has $\int \mathrm{d} t\left|\psi\left(x_{\mathrm{A}}, t\right)\right|^{2}=$ $1 /\left\langle v^{-1}\right\rangle$. The expansion of $\Pi_{\mathrm{K}}$ to second order in $\left(\hat{p}-p_{0}\right)$ yields the flux at $x=x_{\mathrm{A}}$

$$
\Pi_{\mathrm{K}}\left(t, x_{\mathrm{A}}\right)=J\left(x_{\mathrm{A}}, t\right)+\mathcal{O}\left(\left(\hat{p}-p_{0}\right)^{2}\right) .
$$


So, the quantum mechanical flux and the quite intuitive expression of Equation (4.52) can be seen as approximations to the ideal distribution of Kijowski for wave packets peaked sharply in momentum space. 


\section{Chapter 5}

\section{The atomic mirror as a measuring device}

In the following the detection procedure used in the experiment described in Section 4.1 is analyzed in detail. This is done in a simplified one dimensional model without gravitation as depicted in Figure 5.1. Consider a freely moving wave packet prepared at time $t=0$ at position $x_{0}$ coming in from the left. At time $T$ a perfectly reflecting mirror is switched on at the position $x=0$ and switched off again at time $T+\tau$. Then the norm of the reflected part of wave packet is measured. This measurement is done sufficiently far away from the position of the mirror so that the reflected and the transmitted part of the wave packet can be distinguished clearly. For simplicity the mirror will be modeled in the following by applying the boundary condition $\psi(x=0)=0$. This situation can be seen as the limiting case of a square potential of the form

$$
V(x)=V_{0} \chi_{[-a, a]}(x),
$$

where $\chi_{\left[\xi_{1}, \xi_{2}\right]}(x)$ is the characteristic function of the interval $\left[\xi_{1}, \xi_{2}\right]$ and, in the limit $a \rightarrow 0, V_{0}$ has to fulfill $2 V_{0} a \rightarrow \infty$. For example, one could use $V_{0}=U / 2 a^{2}$ with a constant $U$. In other words one needs a potential which is stronger than a deltatype potential. The truncated exponential potential of equation (4.37) approaches the situation described by the above boundary condition for $\kappa \rightarrow \infty$ with $\beta=k_{\max } / \kappa$ kept constant. The approximation is good if the wave packet is prepared such that it is much broader than the decay length $\kappa^{-1}$ of the actual mirror.

\subsection{Calculation of the reflected norm}

In order to investigate which quantities connected with the wave function can be obtained by the measurement scheme described above one has to calculate the norm of the reflected part of the wave packet. There are two different ways of achieving this. In the first method one calculates the momentum amplitude of the wave packet after the mirror has been switched off by projecting onto the corresponding 

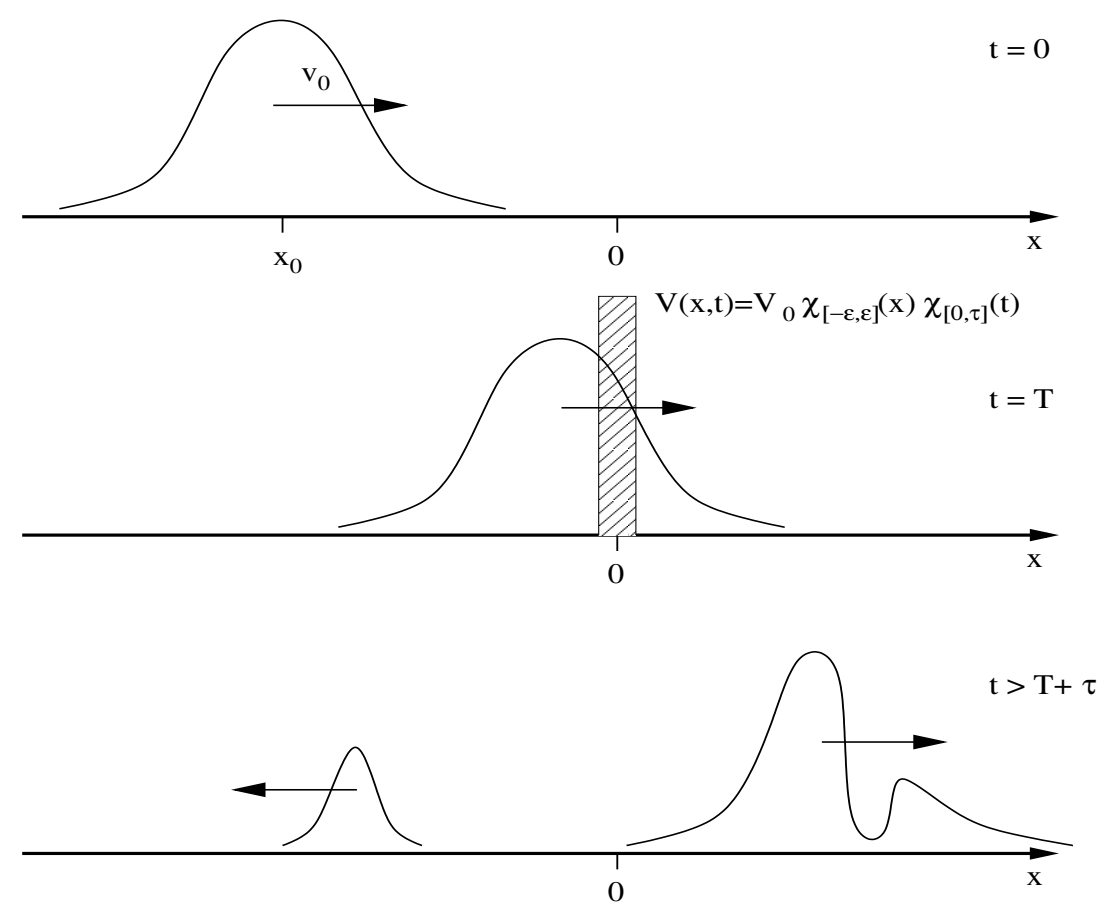

Figure 5.1: Simplified model for the detection scheme of the experiment of Reference [49]. A wave packet prepared at some point $x_{0}<0$ and moving to the right is considered. At $t=T$ a mirror consisting of a thin square well potential with large $V_{0}$ is switched on for a time $\tau$. Afterwards the wave packet again evolves freely and the norm of the reflected part is measured.

momentum eigenstates. The second method uses the Green's function of the problem in order to derive an expression for the wave function in position space after switching off the mirror. Both methods shed light on different aspects of the procedure so they will be presented both.

\subsubsection{Momentum representation point of view}

The momentum amplitude of the incoming wave packet is supposed to have support only for positive momenta. The general case of a wave packet with arbitrary momentum components is discussed later. For a freely moving wave packet the eigenstates can be divided into right and left moving plane waves

$$
\phi_{k}^{+}(x)=\frac{1}{\sqrt{2 \pi}} \mathrm{e}^{\mathrm{i} k x}, \quad \phi_{k}^{-}(x)=\frac{1}{\sqrt{2 \pi}} \mathrm{e}^{-\mathrm{i} k x} .
$$

After the mirror has been switched on one needs new eigenfunctions which account for the boundary condition $\psi=0$ at $x=0$. These are

$$
\phi_{k}^{\mathrm{R}}(x)=\sqrt{\frac{2}{\pi}} \sin (k x) \theta(x), \quad \phi_{k}^{\mathrm{L}}(x)=\sqrt{\frac{2}{\pi}} \sin (k x) \theta(-x) .
$$


for the left and the right hand side. The scalar products between these functions can be easily calculated yielding

$$
\begin{aligned}
\left\langle\phi_{k}^{\mathrm{L}}, \phi_{k^{\prime}}^{+}\right\rangle & =\frac{\mathrm{i}}{2} \delta\left(k-k^{\prime}\right)-\frac{1}{\pi} \mathscr{P} \frac{k}{k^{2}-k^{2}}, \\
\left\langle\phi_{k}^{\mathrm{R}}, \phi_{k^{\prime}}^{+}\right\rangle & =\frac{\mathrm{i}}{2} \delta\left(k-k^{\prime}\right)+\frac{1}{\pi} \mathscr{P} \frac{k}{k^{2}-k^{\prime 2}}, \\
\left\langle\phi_{k}^{-}, \phi_{k^{\prime}}^{\mathrm{L}}\right\rangle & =\frac{\mathrm{i}}{2} \delta\left(k-k^{\prime}\right)+\frac{1}{\pi} \mathscr{P} \frac{k^{\prime}}{k^{2}-k^{\prime 2}}, \\
\left\langle\phi_{k}^{-}, \phi_{k^{\prime}}^{\mathrm{R}}\right\rangle & =-\frac{\mathrm{i}}{2} \delta\left(k-k^{\prime}\right)+\frac{1}{\pi} \mathscr{P} \frac{k^{\prime}}{k^{2}-k^{\prime 2}},
\end{aligned}
$$

where $\mathscr{P}$. denotes the Cauchy principal value. One now starts with a wave function

$$
\psi(x, t)=\int_{0}^{\infty} \mathrm{d} k \widetilde{\psi}(k) \phi_{k}^{+} \mathrm{e}^{-\mathrm{i} \frac{\hbar k^{2}}{2 m} t}
$$

which has been prepared at time $t=0$ at a position $x_{0}$ far away from the mirror. At time $t=T$ the mirror is switched on. Subsequently the parts of the wave packet on the left hand side and on the right hand side of the mirror evolve independently according the eigenstates $\phi_{k}^{\mathrm{R}}(x)$ and $\phi_{k}^{\mathrm{L}}(x)$, with the new momentum amplitudes given by the projections onto these eigenstates, respectively. Therefore, when the mirror is switched off again at a time $T+\tau$, the state at that moment is given by

$$
\psi_{\text {mirr. }}^{\mathrm{L}}(x, T+\tau)=\int_{0}^{\infty} \mathrm{d} k \phi_{k}^{\mathrm{L}} \mathrm{e}^{-\mathrm{i} \frac{\hbar k^{2}}{2 m} \tau} \int_{0}^{\infty} \mathrm{d} k^{\prime}\left\langle\phi_{k}^{\mathrm{L}}, \phi_{k^{\prime}}^{+}\right\rangle \widetilde{\psi}\left(k^{\prime}\right) \mathrm{e}^{-\mathrm{i} \frac{\hbar k^{\prime 2}}{2 m} T}
$$

and

$$
\psi_{\text {mirr. }}^{\mathrm{R}}(x, T+\tau)=\int_{0}^{\infty} \mathrm{d} k \phi_{k}^{\mathrm{R}} \mathrm{e}^{-\mathrm{i} \frac{\hbar k^{2}}{2 m} \tau} \int_{0}^{\infty} \mathrm{d} k^{\prime}\left\langle\phi_{k}^{\mathrm{R}}, \phi_{k^{\prime}}^{+}\right\rangle \widetilde{\psi}\left(k^{\prime}\right) \mathrm{e}^{-\mathrm{i} \frac{\hbar k^{\prime 2}}{2 m} T}
$$

for the left and right hand side of the mirror, respectively. The momentum amplitude of the reflected part of the wave function after switching off the mirror is then obtained by projection onto the free, left moving plane waves

$$
\begin{aligned}
& \widetilde{\psi}_{\text {refl. }}^{\mathrm{L}}(k)=\left\langle\phi_{k}^{-}, \psi_{\text {refl. }}^{\mathrm{L}}\right\rangle=\int_{0}^{\infty} \mathrm{d} k^{\prime}\left\langle\phi_{k}^{-}, \phi_{k^{\prime}}^{\mathrm{L}}\right\rangle \mathrm{e}^{-\mathrm{i} \frac{\hbar k^{\prime 2}}{2 m} \tau} \int_{0}^{\infty} \mathrm{d} k^{\prime \prime}\left\langle\phi_{k^{\prime}}^{\mathrm{L}}, \phi_{k^{\prime \prime}}^{+}\right\rangle \widetilde{\psi}\left(k^{\prime \prime}\right) \mathrm{e}^{-\mathrm{i} \frac{\hbar k^{\prime \prime 2}}{2 m} T}, \\
& \widetilde{\psi}_{\text {refl. }}^{\mathrm{R}}(k)=\left\langle\phi_{k}^{-}, \psi_{\text {refl. }}^{\mathrm{R}}\right\rangle=\int_{0}^{\infty} \mathrm{d} k^{\prime}\left\langle\phi_{k}^{-}, \phi_{k^{\prime}}^{\mathrm{R}}\right\rangle \mathrm{e}^{-\mathrm{i} \frac{\hbar k^{\prime 2}}{2 m} \tau} \int_{0}^{\infty} \mathrm{d} k^{\prime \prime}\left\langle\phi_{k^{\prime}}^{\mathrm{R}}, \phi_{k^{\prime \prime}}^{+}\right\rangle \widetilde{\psi}\left(k^{\prime \prime}\right) \mathrm{e}^{-\mathrm{i} \frac{\hbar k^{\prime \prime 2}}{2 m} T} .
\end{aligned}
$$

After insertion of the identities (5.4) equation (5.8) can be written as

$$
\begin{aligned}
& \widetilde{\psi}_{\text {refl. }}^{\mathrm{L}}(k)=-\frac{1}{4} \widetilde{\psi}(k) \mathrm{e}^{-\mathrm{i} \frac{\hbar k^{2}}{2 m}(T+\tau)}+\frac{\mathrm{i}}{2 \pi} \mathscr{P} \int_{0}^{\infty} \mathrm{d} k^{\prime} \widetilde{\psi}\left(k^{\prime}\right) \frac{k^{\prime}}{k^{2}-k^{\prime 2}} \mathrm{e}^{-\mathrm{i} \frac{\hbar k^{\prime 2}}{2 m}(T+\tau)} \\
& -\frac{\mathrm{i}}{2 \pi} \mathrm{e}^{-\mathrm{i} \frac{\hbar k^{2}}{2 m} \tau} \mathscr{P} \int_{0}^{\infty} \mathrm{d} k^{\prime} \widetilde{\psi}\left(k^{\prime}\right) \frac{k}{k^{2}-k^{\prime 2}} \mathrm{e}^{-\mathrm{i} \frac{\hbar k^{\prime 2}}{2 m} T} \\
& -\frac{1}{\pi^{2}} \mathscr{P} \int_{0}^{\infty} \mathrm{d} k^{\prime} \frac{k^{\prime}}{k^{2}-k^{2}} \mathrm{e}^{-\mathrm{i} \frac{\hbar k^{\prime 2}}{2 m} \tau} \mathscr{P} \int_{0}^{\infty} \mathrm{d} k^{\prime \prime} \widetilde{\psi}\left(k^{\prime \prime}\right) \frac{k^{\prime}}{k^{\prime 2}-k^{\prime \prime 2}} \mathrm{e}^{-\mathrm{i} \frac{\hbar k^{\prime \prime 2}}{2 m} T}
\end{aligned}
$$


for the part left of the mirror. Defining the functions $f_{T}(k)$ and $f_{T+\tau}(k)$ as

$$
f_{T}(k)=\widetilde{\psi}(k) \mathrm{e}^{-\mathrm{i} \frac{\hbar k^{2}}{2 m} T}, \quad \quad f_{T+\tau}(k)=\widetilde{\psi}(k) \mathrm{e}^{-\mathrm{i} \frac{\hbar k^{2}}{2 m}(T+\tau)}
$$

and their Hilbert transforms (see Appendix C) as

$$
\begin{gathered}
g_{T}(k)=H\left[f_{T}\right]=\frac{1}{\pi} \mathscr{P} \int_{-\infty}^{\infty} \mathrm{d} k^{\prime} \frac{f_{T}\left(k^{\prime}\right)}{k^{\prime}-k}, \\
g_{T+\tau}(k)=H\left[f_{T+\tau}\right]=\frac{1}{\pi} \mathscr{P} \int_{-\infty}^{\infty} \mathrm{d} k^{\prime} \frac{f_{T+\tau}\left(k^{\prime}\right)}{k^{\prime}-k}
\end{gathered}
$$

and expanding the fractions one can rewrite Equation (5.10) as

$$
\begin{aligned}
& \widetilde{\psi}_{\text {refl. }}^{\mathrm{L}}(k)=-\frac{1}{4} f_{T+\tau}(k)-\frac{\mathrm{i}}{4 \pi} \mathscr{P} \int_{0}^{\infty} \mathrm{d} k^{\prime} f_{T+\tau}\left(k^{\prime}\right)\left[\frac{1}{k^{\prime}-k}+\frac{1}{k^{\prime}+k}\right] \\
& +\frac{\mathrm{i}}{4 \pi} \mathrm{e}^{-\mathrm{i} \frac{\hbar k^{2}}{2 m} \tau} \mathscr{P} \int_{0}^{\infty} \mathrm{d} k^{\prime} f_{T}\left(k^{\prime}\right)\left[\frac{1}{k^{\prime}-k}-\frac{1}{k^{\prime}+k}\right] \\
& -\frac{1}{4 \pi^{2}} \mathscr{P} \int_{0}^{\infty} \mathrm{d} k^{\prime}\left[\frac{1}{k^{\prime}-k}+\frac{1}{k^{\prime}+k}\right] \mathrm{e}^{-\mathrm{i} \frac{\hbar k^{\prime 2}}{2 m} \tau} \times \\
& \times \mathscr{P} \int_{0}^{\infty} \mathrm{d} k^{\prime \prime} f_{T}\left(k^{\prime \prime}\right)\left[\frac{1}{k^{\prime \prime}-k^{\prime}}-\frac{1}{k^{\prime \prime}+k^{\prime}}\right] .
\end{aligned}
$$

Since $f_{t}(k)=0$ for $k \leq 0$, the integrals containing $f$ can be expanded over the whole real axis. Therefore one has

$$
\begin{aligned}
\widetilde{\psi}_{\text {refl. }}^{\mathrm{L}}(k)= & -\frac{1}{4} f_{T+\tau}(k)-\frac{1}{4 \pi} \mathscr{P} \int_{-\infty}^{\infty} \mathrm{d} k^{\prime} \frac{g_{T}\left(k^{\prime}\right)-g_{T}\left(-k^{\prime}\right)}{k^{\prime}-k} \mathrm{e}^{-\mathrm{i} \frac{\hbar k^{\prime 2}}{2 m} \tau} \\
& -\frac{\mathrm{i}}{4}\left[g_{T+\tau}(k)+g_{T+\tau}(-k)\right]+\frac{\mathrm{i}}{4}\left[g_{T}(k)-g_{T}(-k)\right] \mathrm{e}^{-\mathrm{i} \frac{\hbar k^{2}}{2 m} \tau} .
\end{aligned}
$$

It is important to note here that $g_{t}(k)$ spreads over the whole momentum range, although $f_{t}(k)$ is only non-zero for positive values of $k$. In order to get some physical insight into this expression it is useful to consider the limit $\tau \rightarrow 0$. This corresponds to cutting off at time $T$ the part of the wave packet which is on the right hand side of the mirror. With the skew-reciprocity of the Hilbert transform $H[H[f]]=-f$ $[95,96]$, the second term in Equation $(5.15)$ becomes $1 / 4\left(f_{T}(k)+f_{T}(-k)\right)$ and one has

$$
\widetilde{\psi}_{\text {reff. }}^{\mathrm{L}}(k)=\frac{1}{4} f_{T}(-k)-\frac{\mathrm{i}}{2} g_{T}(-k) .
$$

The first term is zero for positive $k$ but the second term still remains. So, there is some reflection even though the mirror was not switched on. This is due to the fact that a sudden cut-off of a wave function leads to back flow even if the original wave packet only had positive momentum components. 
An analogous calculation for the part of the wave function on the right hand side of the mirror gives

$$
\begin{aligned}
\widetilde{\psi}_{\text {refl. }}^{\mathrm{R}}(k)= & -\frac{1}{4} f_{T+\tau}(k)-\frac{1}{4 \pi} \mathscr{P} \int_{-\infty}^{\infty} \mathrm{d} k^{\prime} \frac{g_{T}\left(k^{\prime}\right)-g_{T}\left(-k^{\prime}\right)}{k^{\prime}-k} \mathrm{e}^{-\mathrm{i} \frac{\hbar k^{\prime 2}}{2 m} \tau} \\
& +\frac{\mathrm{i}}{4}\left[g_{T+\tau}(k)+g_{T+\tau}(-k)\right]-\frac{\mathrm{i}}{4}\left[g_{T}(k)-g_{T}(-k)\right] \mathrm{e}^{-\mathrm{i} \frac{\hbar k^{2}}{2 m} \tau} .
\end{aligned}
$$

In this expression also a small reflected amplitude remains in the limit $\tau \rightarrow 0$. If one is interested in the overall reflected amplitude one has to add up Equations (5.15) and (5.17) which leads to

$$
\widetilde{\psi}_{\text {refl. }}(k)=-\frac{1}{2} f_{T+\tau}(k)-\frac{1}{2 \pi} \mathscr{P} \int_{-\infty}^{\infty} \mathrm{d} k^{\prime} \frac{g_{T}\left(k^{\prime}\right)-g_{T}\left(-k^{\prime}\right)}{k^{\prime}-k} \mathrm{e}^{-\mathrm{i} \frac{\hbar k^{\prime 2}}{2 m} \tau}
$$

In this expression the second lines of Equations (5.15) and (5.17) have canceled out and for $\tau \rightarrow 0$ one indeed has no reflection. This corresponds to the physical picture that no cut-off has been applied and the wave packet moves freely across the site of the mirror. By looking at equation (5.17) one might get the impression that the reflected amplitude from the right hand side of the mirror is similar to the reflected amplitude which originates from the left hand side since the corresponding expressions are quite similar. In fact, the important difference between the two expressions is the different sign of the second row. For the right hand side the first and the second line cancel each other out, so that only a very small amplitude remains which is due to the cut-off effect mentioned above.

One may ask whether Equation (5.15) or equation (5.18) provides the more realistic description of the experiment. If the mirror is switched off in the experiment the atoms hit the prism and stick to the surface or are scattered diffusively. In either case they are lost to the experiment and do not appear in the reflected amplitude. Therefore, considering the whole reflected amplitude as in Equation (5.18) seems more appropriate since only for this case there is no reflection in the limit $\tau \rightarrow 0$ which corresponds to the mirror never being switched on. Also, the actual reflection from the exponential potential happens at a distance of approximately $1 / \kappa$ in front of the prism surface. So one may argue that also in the experiment there is the possibility of having a small part of the reflected amplitude coming from the right hand side of the actual point of reflection.

For sake of completeness the expressions for the momentum amplitude of the transmitted part of the wave function are also given which are

$$
\begin{aligned}
\widetilde{\psi}_{\text {trans. }}^{\mathrm{L}}(k)= & \frac{1}{4} f_{T+\tau}(k)-\frac{1}{4 \pi} \mathscr{P} \int_{-\infty}^{\infty} \mathrm{d} k^{\prime} \frac{g_{T}\left(k^{\prime}\right)-g_{T}\left(-k^{\prime}\right)}{k^{\prime}-k} \mathrm{e}^{-\mathrm{i} \frac{\hbar k^{\prime 2}}{2 m} \tau} \\
& -\frac{\mathrm{i}}{4}\left[g_{T+\tau}(k)+g_{T+\tau}(-k)\right]-\frac{\mathrm{i}}{4}\left[g_{T}(k)-g_{T}(-k)\right] \mathrm{e}^{-\mathrm{i} \frac{\hbar k^{2}}{2 m} \tau} .
\end{aligned}
$$


for the part on the left hand side of the mirror and

$$
\begin{aligned}
\widetilde{\psi}_{\text {trans. }}^{\mathrm{R}}(k)= & \frac{1}{4} f_{T+\tau}(k)-\frac{1}{4 \pi} \mathscr{P} \int_{-\infty}^{\infty} \mathrm{d} k^{\prime} \frac{g_{T}\left(k^{\prime}\right)-g_{T}\left(-k^{\prime}\right)}{k^{\prime}-k} \mathrm{e}^{-\mathrm{i} \frac{\hbar k^{\prime 2}}{2 m} \tau} \\
& +\frac{\mathrm{i}}{4}\left[g_{T+\tau}(k)+g_{T+\tau}(-k)\right]+\frac{\mathrm{i}}{4}\left[g_{T}(k)-g_{T}(-k)\right] \mathrm{e}^{-\mathrm{i} \frac{\hbar k^{2}}{2 m} \tau}
\end{aligned}
$$

for the part on the right hand side of the mirror. This yields

$$
\widetilde{\psi}_{\text {trans. }}(k)=\frac{1}{2} f_{T+\tau}(k)-\frac{1}{2 \pi} \mathscr{P} \int_{-\infty}^{\infty} \mathrm{d} k^{\prime} \frac{g_{T}\left(k^{\prime}\right)-g_{T}\left(-k^{\prime}\right)}{k^{\prime}-k} \mathrm{e}^{-\mathrm{i} \frac{\hbar k^{\prime 2}}{2 m} \tau}
$$

for the whole transmitted wave packet. As expected this last expression tends to $f_{T}(k)$, i.e. the momentum amplitude of the free wave packet, for $\tau \rightarrow 0$.

\subsubsection{Green's function point of view}

The momentum amplitudes obtained in the previous section can also be calculated using the Green's function of the Schrödinger equation in position space. This allows a different view on the results of the last section.

The Green's function of the Schrödinger equation for a free particle is given by

$$
G_{t}^{\mathrm{F}}\left(x, x^{\prime}\right)=\sqrt{\frac{m}{2 \pi \mathrm{i} \hbar t}} \mathrm{e}^{-\frac{m}{2 \pi \mathrm{i} \hbar t}\left(x-x^{\prime}\right)^{2}} .
$$

From this one can easily calculate the Green's function for a free particle with a mirror at $x=0$, i.e., for the boundary condition $\psi(0, t)=0$

$$
G_{t}^{\mathrm{S}}\left(x, x^{\prime}\right)=\sqrt{\frac{m}{2 \pi \mathrm{i} \hbar t}}\left[\mathrm{e}^{-\frac{m}{2 \mathrm{i} \hbar t}\left(x-x^{\prime}\right)^{2}}-\mathrm{e}^{-\frac{m}{2 \mathrm{i} \hbar t}\left(x+x^{\prime}\right)^{2}}\right] .
$$

With this Green's function it is possible to calculate the time development of a wave function subject to the mirroring procedure described above. Namely, the wave function for $x>0$ at the switch-off time of the mirror is given by

$$
\begin{aligned}
\psi_{T+\tau}^{\mathrm{R}}(x) & =\theta(x) \int_{0}^{\infty} \mathrm{d} x^{\prime} G_{\tau}^{\mathrm{S}}\left(x, x^{\prime}\right) \psi_{T}\left(x^{\prime}\right) \\
& =\theta(x) \sqrt{\frac{m}{2 \pi \mathrm{i} \hbar t}}\left[\int_{0}^{\infty} \mathrm{d} x^{\prime} \mathrm{e}^{-\frac{m}{2 i \hbar t}\left(x-x^{\prime}\right)^{2}} \psi_{T}\left(x^{\prime}\right)-\int_{0}^{\infty} \mathrm{d} x^{\prime} \mathrm{e}^{-\frac{m}{2 i \hbar \hbar}\left(x+x^{\prime}\right)^{2}} \psi_{T}\left(x^{\prime}\right)\right],
\end{aligned}
$$

where again $\theta(x)$ denotes the Heaviside step function and $\psi_{T}\left(x^{\prime}\right)$ is the wave function at the switch-on time $T$ which is connected to the wave function at preparation time $t=0$ via

$$
\psi_{T}(x)=\int_{\infty}^{\infty} \mathrm{d} x^{\prime} G_{T}^{\mathrm{F}}\left(x, x^{\prime}\right) \psi\left(x^{\prime}, 0\right)
$$


Analogously, the wave function for $x<0$ is given by

$$
\psi_{T+\tau}^{\mathrm{L}}(x)=\theta(-x) \sqrt{\frac{m}{2 \pi \mathrm{i} \hbar t}}\left[\int_{-\infty}^{0} \mathrm{~d} x^{\prime} \mathrm{e}^{-\frac{m}{2 \mathrm{i} \hbar t}\left(x-x^{\prime}\right)^{2}} \psi_{T}\left(x^{\prime}\right)-\int_{-\infty}^{0} \mathrm{~d} x^{\prime} \mathrm{e}^{-\frac{m}{2 \mathrm{i} \hbar t}\left(x+x^{\prime}\right)^{2}} \psi_{T}\left(x^{\prime}\right)\right] .
$$

The above formulas can be rewritten in a compact form using the convolution formula

$$
f * g=\int_{-\infty}^{\infty} \mathrm{d} x^{\prime} f\left(x-x^{\prime}\right) g\left(x^{\prime}\right) .
$$

This yields

$$
\psi_{T+\tau}^{\mathrm{R}}(x)=\theta\left(G_{\tau}^{\mathrm{F}} *\left(\theta \psi_{T}\right)\right)(x)-\theta\left(G_{\tau}^{\mathrm{F}} * \pi\left(\theta \psi_{T}\right)\right)(x),
$$

where $\pi(\cdot)$ denotes the parity operation $x \rightarrow-x$. Analogously, the wave function on the left hand side can be written as

$$
\psi_{T+\tau}^{\mathrm{L}}(x)=\theta_{-}\left(G_{\tau}^{\mathrm{F}} *\left(\theta_{-} \psi_{T}\right)\right)(x)-\theta_{-}\left(G_{\tau}^{\mathrm{F}} * \pi\left(\theta_{-} \psi_{T}\right)\right)(x),
$$

with $\theta_{-}=\theta(-x)$. Finally, the overall wave function at switch-off time is given by the sum of the two terms

$$
\psi_{T+\tau}(x)=\psi_{T+\tau}^{\mathrm{L}}(x)+\psi_{T+\tau}^{\mathrm{R}}(x) .
$$

As in the previous section one can take the limit $\tau \rightarrow 0$ which implies $G_{\tau}^{\mathrm{F}} \rightarrow \delta$. In this limit one has

$$
\begin{aligned}
& \theta\left(\delta *\left(\theta \psi_{T}\right)\right)(x)-\theta\left(\delta * \pi\left(\theta \psi_{T}\right)\right)(x)+\theta_{-}\left(\delta *\left(\theta_{-} \psi_{T}\right)\right)(x)-\theta_{-}\left(\delta * \pi\left(\theta_{-} \psi_{T}\right)\right)(x) \\
& =\left(\theta \psi_{T}\right)(x)-\left(\theta \theta_{-} \psi_{T}\right)(x)+\left(\theta_{-} \psi_{T}\right)(x)-\left(\theta_{-} \theta \psi_{T}\right)(x)=\psi_{T}(x)
\end{aligned}
$$

as expected.

In order to identify the left moving part of the wave packet one can now go to momentum space. With the use of the convolution formulas

$$
\widetilde{f * g}=\sqrt{2 \pi} \widetilde{f} \widetilde{g} \quad \text { and } \quad \widetilde{f} * \widetilde{g}=\sqrt{2 \pi \widetilde{f g}},
$$

where $\widetilde{\cdot}$ denotes the Fourier transform, one has

$$
\begin{aligned}
\widetilde{\psi}_{T+\tau}(k)=\frac{1}{\sqrt{2 \pi}}[ & {\left[\widetilde{\theta} *\left(\widetilde{G}_{\tau}^{\mathrm{F}}\left(\widetilde{\theta} * \widetilde{\psi}_{T}\right)\right)(k)-\widetilde{\theta} *\left(\widetilde{G}_{\tau}^{\mathrm{F}} \pi\left(\widetilde{\theta} * \widetilde{\psi}_{T}\right)\right)(k)\right.} \\
& \left.+\widetilde{\theta}_{-} *\left(\widetilde{G}_{\tau}^{\mathrm{F}}\left(\widetilde{\theta}_{-} * \widetilde{\psi}_{T}\right)\right)(k)-\widetilde{\theta}_{-} *\left(\widetilde{G}_{\tau}^{\mathrm{F}} \pi\left(\widetilde{\theta}_{-} * \widetilde{\psi}_{T}\right)\right)(k)\right] .
\end{aligned}
$$

The Fourier transforms of the functions involved in this formula can be easily calculated yielding

$$
\widetilde{G}_{\tau}^{\mathrm{F}}(k)=\frac{1}{\sqrt{2 \pi}} \mathrm{e}^{-\mathrm{i} \frac{\hbar k^{2}}{2 m} \tau}, \quad \widetilde{\theta}(k)=\frac{1}{\sqrt{2 \pi}} \frac{1}{\mathrm{i} k+\epsilon}, \quad \text { and } \quad \widetilde{\theta}_{-}(k)=-\frac{1}{\sqrt{2 \pi}} \frac{1}{\mathrm{i} k-\epsilon} .
$$


Inserting these Fourier transforms into Equation (5.31) yields

$$
\widetilde{\psi}_{T+\tau}^{\mathrm{L}}(k)=\frac{1}{4 \pi^{2}} \int_{-\infty}^{\infty} \mathrm{d} k^{\prime}\left[\frac{1}{\mathrm{i}\left(k-k^{\prime}\right)-\epsilon}-\frac{1}{\mathrm{i}\left(k+k^{\prime}\right)-\epsilon}\right] \mathrm{e}^{-\mathrm{i} \alpha k^{\prime 2}} \int_{-\infty}^{\infty} \mathrm{d} k^{\prime \prime} \frac{\widetilde{\psi}_{T}\left(k^{\prime \prime}\right)}{\mathrm{i}\left(k^{\prime}-k^{\prime \prime}\right)-\epsilon}
$$

for the momentum amplitude resulting from the left hand side and

$$
\widetilde{\psi}_{T+\tau}^{\mathrm{R}}(k)=\frac{1}{4 \pi^{2}} \int_{-\infty}^{\infty} \mathrm{d} k^{\prime}\left[\frac{1}{\mathrm{i}\left(k-k^{\prime}\right)+\epsilon}-\frac{1}{\mathrm{i}\left(k+k^{\prime}\right)+\epsilon}\right] \mathrm{e}^{-\mathrm{i} \alpha k^{\prime 2}} \int_{-\infty}^{\infty} \mathrm{d} k^{\prime \prime} \frac{\widetilde{\psi}_{T}\left(k^{\prime \prime}\right)}{\mathrm{i}\left(k^{\prime}-k^{\prime \prime}\right)+\epsilon}
$$

for the momentum amplitude resulting from the right hand side. Here the quantity $\alpha=\frac{\hbar \tau}{2 m}$ was used as a simplification. Note that these are the momentum amplitudes for the whole wave function and are therefore valid for both the reflected (negative $k$ ) and the transmitted (positive $k$ ) part. The expressions of equations $(5.15,5.17,5.18)$ and $(5.19,5.20,5.21)$ are obtained by inserting the identities

$$
\begin{aligned}
& \frac{1}{\mathrm{i}\left(k \pm k^{\prime}\right)-\epsilon}=-\mathrm{i} \mathscr{P} \frac{1}{k \pm k^{\prime}}+\pi \delta\left(k \pm k^{\prime}\right) \\
& \frac{1}{\mathrm{i}\left(k \pm k^{\prime}\right)+\epsilon}=-\mathrm{i} \mathscr{P} \frac{1}{k \pm k^{\prime}}-\pi \delta\left(k \pm k^{\prime}\right)
\end{aligned}
$$

and substituting $k \rightarrow-k$ for the reflected parts. Also note that at this point of the calculation the momentum amplitude at switch-on time $\widetilde{\psi}_{T}(k)$ is not restricted to positive $k$-values but allows for general amplitudes.

\subsection{Behavior for short switch-on times of the mir- ror}

In order to get more physical insight into the expressions obtained in the previous section it is helpful to look at the behavior of the norm of the reflected wave packet in the case when the mirror is switched on for only a very short time. This will be done by expanding the momentum amplitudes in $\alpha=\frac{\hbar \tau}{2 m}$ which occurs as $\alpha k^{2}$ in the exponential. Therefore the condition $\tau \ll 2 m / \hbar k_{0}^{2}$, where $p_{0}=\hbar k_{0}$ is the mean momentum of the wave packet, has to be fulfilled in order to have a valid expansion in $\alpha$.

The expansion is done for the model without a cut-off of the wave packet. One therefore has to consider the sum of equations (5.33) and (5.34). In addition, wave packets with only positive momentum components before the measurement, i.e. $\widetilde{\psi}_{T}(k)=0$ for $k<0$, are regarded now. The general case of wave packets with arbitrary momentum components is discussed further below. When adding up Equations (5.33) and (5.34) and inserting the identities (5.35) the mixed terms - the terms containing a principal value in the first integral and a delta function in the 
second or vice versa- drop out and one has

$$
\begin{aligned}
& \widetilde{\psi}_{T+\tau}(k)=\frac{1}{2} \int_{-\infty}^{\infty} \mathrm{d} k^{\prime}\left(\delta\left(k-k^{\prime}\right)-\delta\left(k+k^{\prime}\right)\right) \mathrm{e}^{-\mathrm{i} \alpha k^{\prime 2}} \int_{-\infty}^{\infty} \mathrm{d} k^{\prime \prime} \delta\left(k^{\prime}-k^{\prime \prime}\right) \widetilde{\psi}_{T}\left(k^{\prime \prime}\right) \\
& +\frac{1}{2 \pi^{2}} \int_{-\infty}^{\infty} \mathrm{d} k^{\prime}\left[\mathscr{P} \frac{1}{k^{\prime}-k}+\mathscr{P} \frac{1}{k^{\prime}+k}\right] \mathrm{e}^{-\mathrm{i} \alpha k^{\prime 2}} \mathscr{P} \int_{-\infty}^{\infty} \mathrm{d} k^{\prime \prime} \frac{\widetilde{\psi}_{T}\left(k^{\prime \prime}\right)}{k^{\prime}-k^{\prime \prime}} \\
& =\frac{1}{2}\left(\widetilde{\psi}_{T}(k)-\widetilde{\psi}_{T}(-k)\right) \mathrm{e}^{-\mathrm{i} \alpha k^{2}} \\
& +\frac{1}{2 \pi^{2}} \mathscr{P} \int_{-\infty}^{\infty} \mathrm{d} k^{\prime}\left[\frac{1}{k^{\prime}-k}+\frac{1}{k^{\prime}+k}\right] \mathrm{e}^{-\mathrm{i} \alpha k^{\prime 2}} \mathscr{P} \int_{-\infty}^{\infty} \mathrm{d} k^{\prime \prime} \frac{\widetilde{\psi}_{T}\left(k^{\prime \prime}\right)}{k^{\prime}-k^{\prime \prime}}
\end{aligned}
$$

as the total momentum amplitude after switching off the mirror. This result can already be used to calculate the first order term of the expansion. In the experiment the norm in position space of the reflected part of the wave packet is measured at a time $t_{\text {meas. }}$ at which it is again far away from the mirror. This is equivalent to measuring in momentum space the part of the norm of the wave packet with negative $k$ at switch-off time

$$
N_{\text {refl. }}(\alpha)=\int_{-\infty}^{\infty} \mathrm{d} x\left|\psi_{\text {refl. }}\left(x, T+\tau+t_{\text {meas. }}\right)\right|^{2}=\int_{-\infty}^{0} \mathrm{~d} k\left|\widetilde{\psi}_{T+\tau}(k)\right|^{2} .
$$

Note that for the momentum amplitude obtained from the Green's function picture the reflected part of the wave function indeed corresponds to negative values of $k$ whereas in the projection picture the same quantity was given with positive momenta since it was obtained by projecting on the left moving plane waves proportional to $\mathrm{e}^{-\mathrm{i} k x}$.

\section{Calculation of the lowest order term}

In order to compute the expansion in $\alpha$ of equation (5.37) one has to keep in mind that only the linear combination of the two terms in Equation (5.36) tends to zero for $\tau \rightarrow 0$ and not the individual terms themselves. Therefore it is useful to incorporate the free momentum distribution terms into the integral. This can be achieved my means of the identity

$$
\int_{-\infty}^{\infty} \mathrm{d} k^{\prime} \mathscr{P} \frac{1}{k^{\prime}-k} \mathscr{P} \frac{1}{k^{\prime}-k^{\prime \prime}}=\pi^{2} \delta\left(k-k^{\prime \prime}\right)
$$

which is a restatement of the skew-reciprocity of the Hilbert transform. Then the first term of Equation (5.36) can be rewritten in the form

$$
\begin{aligned}
& \frac{1}{2}\left(\widetilde{\psi}_{T}(k)-\widetilde{\psi}_{T}(-k)\right) \mathrm{e}^{-\mathrm{i} \alpha k^{2}}=\widetilde{\psi}_{T}(k) \mathrm{e}^{-\mathrm{i} \alpha k^{2}}-\frac{1}{2}\left(\widetilde{\psi}_{T}(k)+\widetilde{\psi}_{T}(-k)\right) \mathrm{e}^{-\mathrm{i} \alpha k^{2}} \\
= & \widetilde{\psi}_{T}(k) \mathrm{e}^{-\mathrm{i} \alpha k^{2}}-\frac{1}{2 \pi^{2}} \mathscr{P} \int_{-\infty}^{\infty} \mathrm{d} k^{\prime}\left[\frac{1}{k^{\prime}-k}+\frac{1}{k^{\prime}+k}\right] \mathscr{P} \int_{-\infty}^{\infty} \mathrm{d} k^{\prime \prime} \frac{\widetilde{\psi}_{T}\left(k^{\prime \prime}\right) \mathrm{e}^{-\mathrm{i} \alpha k^{\prime \prime 2}}}{k^{\prime}-k^{\prime \prime}} .
\end{aligned}
$$


The term $\widetilde{\psi}_{T}(k) \mathrm{e}^{-\mathrm{i} \alpha k^{2}}$ can be omitted since it vanishes on the interval in question. Now equations (5.36) and (5.39) can be combined and the substitution $k^{\prime} \rightarrow k^{\prime} / \sqrt{\alpha}$ can be made. This yields

$$
\begin{array}{r}
\widetilde{\psi}_{\text {refl. }}(k)=\frac{\sqrt{\alpha}}{2 \pi^{2}} \mathscr{P} \int_{-\infty}^{\infty} \mathrm{d} k^{\prime}\left[\frac{1}{k^{\prime}-\sqrt{\alpha} k}+\frac{1}{k^{\prime}+\sqrt{\alpha} k}\right]\left[\mathrm{e}^{-\mathrm{i} k^{\prime 2}} \mathscr{P} \int_{-\infty}^{\infty} \mathrm{d} k^{\prime \prime} \frac{\widetilde{\psi}_{T}\left(k^{\prime \prime}\right)}{k^{\prime}-\sqrt{\alpha} k^{\prime \prime}}\right. \\
\left.-\mathscr{P} \int_{-\infty}^{\infty} \mathrm{d} k^{\prime \prime} \frac{\widetilde{\psi}_{T}\left(k^{\prime \prime}\right) \mathrm{e}^{-\mathrm{i} \alpha k^{\prime \prime 2}}}{k^{\prime}-\sqrt{\alpha} k^{\prime \prime}}\right] .
\end{array}
$$

This formula can be used for the computation of the different orders of the expansion in $\alpha$. When keeping only the lowest order in $\alpha$ the term in the second brackets gives $\left(\mathrm{e}^{-\mathrm{i} k^{\prime 2}}-1\right) / k^{\prime}$. This is an odd function and thus one has

$$
\widetilde{\psi}_{\text {refl. }}(k)=\frac{\sqrt{2 \pi \alpha}}{\pi^{2}} \psi_{T}(x=0) \mathscr{P} \int_{-\infty}^{\infty} \mathrm{d} k^{\prime} \frac{1}{k^{\prime}-\sqrt{\alpha} k} \frac{\mathrm{e}^{-\mathrm{i} k^{\prime 2}}-1}{k^{\prime}}
$$

with

$$
\psi_{T}(x=0)=\frac{1}{\sqrt{2 \pi}} \int_{-\infty}^{\infty} \mathrm{d} k \widetilde{\psi}_{T}(k) .
$$

In order to calculate the norm of the reflected part of the wave packet Equation (5.41) can now be inserted into Equation (5.37) which leads to

$$
\begin{array}{r}
N_{\text {refl. }}(\alpha)=\frac{2 \alpha}{\pi^{3}}\left|\psi_{T}(0)\right|^{2} \int_{-\infty}^{\infty} \mathrm{d} k^{\prime} \int_{-\infty}^{\infty} \mathrm{d} k^{\prime \prime} \int_{-\infty}^{0} \mathrm{~d} k \mathscr{P} \frac{1}{k^{\prime}-\sqrt{\alpha} k} \mathscr{P} \frac{1}{k^{\prime \prime}-\sqrt{\alpha} k} \times \\
\times \frac{\mathrm{e}^{-\mathrm{i} k^{\prime 2}-1}}{k^{\prime}} \frac{\mathrm{e}^{\mathrm{i} k^{\prime \prime 2}}-1}{k^{\prime \prime}} .
\end{array}
$$

Due to the invariance of the integrand under a simultaneous change of sign of all variables, the integration over $k$ just gives an additional factor of two when extended from $-\infty$ to $\infty$. After a further substitution $k \rightarrow k / \sqrt{\alpha}$ one can apply again the identity (5.38), and to lowest order in $\alpha$ the norm of the reflected part of the wave packet is given by

$$
N_{\text {refl. }}(\alpha)=\frac{\sqrt{\alpha}}{\pi}\left|\psi_{T}(0)\right|^{2} \int_{-\infty}^{\infty} \mathrm{d} k \frac{2-2 \cos k^{2}}{k^{2}}=2 \sqrt{\frac{2 \alpha}{\pi}}\left|\psi_{T}(0)\right|^{2}
$$

This is a first major result of the model for the description of the experiment. Namely, for sufficiently short switch-on times of the mirror the norm of the reflected part of the wave packet is proportional to the density at the position $x=0$ of the mirror and at switch-on time $t=T$. This result and further implications of it will be discussed more thoroughly in the next section. 


\section{Higher order terms}

The higher order terms of the norm can be obtained in the same way. For this, the terms in the second pair of brackets of Equation (5.40) have to be expanded in powers of $\sqrt{\alpha}$

$$
\begin{aligned}
\int_{-\infty}^{\infty} \mathrm{d} k^{\prime \prime} \frac{\widetilde{\psi}_{T}\left(k^{\prime \prime}\right)}{k^{\prime}-\sqrt{\alpha} k^{\prime \prime}} & =\frac{1}{k^{\prime}}\left[\int_{-\infty}^{\infty} \mathrm{d} k^{\prime \prime} \widetilde{\psi}_{T}\left(k^{\prime \prime}\right)+\frac{\sqrt{\alpha}}{k^{\prime}} \int_{-\infty}^{\infty} \mathrm{d} k^{\prime \prime} k^{\prime \prime} \widetilde{\psi}_{T}\left(k^{\prime \prime}\right)+\mathcal{O}(\alpha)\right] \\
& =\frac{\sqrt{2 \pi}}{k^{\prime}}\left[\psi_{T}(0)+\frac{\mathrm{i} \sqrt{\alpha}}{k^{\prime}} \psi_{T}^{\prime}(0)-\frac{\alpha}{k^{\prime 2}} \psi_{T}^{\prime \prime}(0)+\mathcal{O}\left(\sqrt{\alpha}^{3}\right)\right]
\end{aligned}
$$

and

$$
\begin{array}{r}
\int_{-\infty}^{\infty} \mathrm{d} k^{\prime \prime} \frac{\widetilde{\psi}_{T}\left(k^{\prime \prime}\right) \mathrm{e}^{-\mathrm{i} \alpha k^{\prime \prime 2}}}{k^{\prime}-\sqrt{\alpha} k^{\prime \prime}}=\frac{1}{k^{\prime}}\left[\int_{-\infty}^{\infty} \mathrm{d} k^{\prime \prime} \widetilde{\psi}_{T}\left(k^{\prime \prime}\right)+\frac{\sqrt{\alpha}}{k^{\prime}} \int_{-\infty}^{\infty} \mathrm{d} k^{\prime \prime} k^{\prime \prime} \widetilde{\psi}_{T}\left(k^{\prime \prime}\right)+\mathcal{O}(\alpha)\right] \\
=\frac{\sqrt{2 \pi}}{k^{\prime}}\left[\psi_{T}(0)+\frac{\mathrm{i} \sqrt{\alpha}}{k^{\prime}} \psi_{T}^{\prime}(0)-\frac{\alpha}{k^{\prime 2}}\left(1+\mathrm{i} k^{\prime 2}\right) \psi_{T}^{\prime \prime}(0)+\mathcal{O}\left(\sqrt{\alpha}^{3}\right)\right] .
\end{array}
$$

As for the first order term the two fractions in Equation (5.40) can be combined by substituting $k^{\prime}$ by $-k^{\prime}$ in the $1 / k^{\prime}+\sqrt{\alpha} k$-term. This cancels the terms with even functions of $k^{\prime}$ in the expansion and leads to

$$
\begin{aligned}
& \widetilde{\psi}_{\text {refl. }}(k)= \\
& \frac{\sqrt{2 \pi \alpha}}{\pi^{2}} \mathscr{P} \int_{-\infty}^{\infty} \mathrm{d} k^{\prime} \frac{1}{k^{\prime}-\sqrt{\alpha} k} \underbrace{\left[\frac{\mathrm{e}^{-\mathrm{i} k^{\prime 2}}-1}{k^{\prime}} \psi_{T}(0)-\alpha \frac{\mathrm{e}^{-\mathrm{i} k^{\prime 2}}-1+\mathrm{i} k^{\prime 2}}{k^{\prime 3}} \psi_{T}^{\prime \prime}(0)+\mathcal{O}\left(\alpha^{2}\right)\right]}_{\equiv f_{\alpha}\left(k^{\prime}\right)} .
\end{aligned}
$$

This can now be inserted into Equation (5.37) and one has

$$
N_{\text {refl. }}(\alpha)=\frac{2 \alpha}{\pi^{3}} \int_{-\infty}^{0} \mathrm{~d} k \int_{-\infty}^{\infty} \mathrm{d} k^{\prime} \int_{-\infty}^{\infty} \mathrm{d} k^{\prime \prime} \mathscr{P} \frac{1}{k^{\prime}-\sqrt{\alpha} k} \mathscr{P} \frac{1}{k^{\prime \prime}-\sqrt{\alpha} k} f_{\alpha}\left(k^{\prime}\right) \overline{f_{\alpha}\left(k^{\prime \prime}\right)} .
$$

By the same argument as above this yields

$$
N_{\text {refl. }}(\alpha)=\frac{\sqrt{\alpha}}{\pi} \int_{-\infty}^{\infty} \mathrm{d} k\left|f_{\alpha}(k)\right|^{2}
$$

and up to third order in $\alpha$ on has

$$
N_{\text {refl. }}(\alpha)=2 \sqrt{\frac{2 \alpha}{\pi}}\left[\left|\psi_{T}(0)\right|^{2}-\alpha\left(\frac{1}{3} \operatorname{Re}\left(\overline{\psi_{T}(0)} \psi_{T}^{\prime \prime}(0)\right)-\operatorname{Im}\left(\overline{\psi_{T}(0)} \psi_{T}^{\prime \prime}(0)\right)\right)\right.
$$




$$
\left.+\alpha^{2}\left(\frac{2}{5}\left|\psi_{T}^{\prime \prime}(0)\right|^{2}-\frac{11}{30} \operatorname{Re}\left(\overline{\psi_{T}(0)} \psi_{T}^{(4)}(0)\right)+\frac{1}{6} \operatorname{Im}\left(\overline{\psi_{T}(0)} \psi_{T}^{(4)}(0)\right)\right)\right] .
$$

Here, $\psi_{T}^{(4)}$ denotes the fourth derivative of $\psi_{T}$ with respect to $x$. So, if the mirror was switched on for a sufficiently short span of time, the signal obtained by the measurement procedure analyzed here is proportional to the density evaluated at the position of the mirror. If one repeats the measurement with a varied switch-on time $T$ one can therefore measure this quantity against time. It is known that it can be seen as a semi-classical arrival-time. Note that an expansion of the wave function in odd powers of $\sqrt{t}$ has recently been obtained in the somewhat related problem of the time evolution of a wave function which at $t=0$ was confined in a region of space with sharp boundaries [97].

\section{The case of arbitrary momentum distributions}

In principal one can also think of a situation in which the wave packet, at the time of preparation, has a momentum amplitude with contributions from negative momenta. In the following the expansion in powers of $\alpha$ of the norm of the reflected part of the wave packet is done for such general momentum amplitudes. In this case the first term in Equation (5.39) has to be taken into account

$$
\begin{aligned}
\widetilde{\psi}_{\text {refl. }}(k) & =\widetilde{\psi}_{T}(k) \mathrm{e}^{-\mathrm{i} \alpha k^{2}}+\frac{\sqrt{2 \pi \alpha}}{\pi^{2}} \int_{-\infty}^{\infty} \mathrm{d} k^{\prime} \mathscr{P} \frac{1}{k^{\prime}-\sqrt{\alpha} k} f_{\alpha}\left(k^{\prime}\right) \\
& \equiv \widetilde{\psi}_{(1)}(k)+\widetilde{\psi}_{(2)}(k) .
\end{aligned}
$$

The reflected norm calculated with this expression then has three contributions

$$
N_{\text {refl. }}(\alpha)=\int_{-\infty}^{0} \mathrm{~d} k\left|\widetilde{\psi}_{(1)}(k)\right|^{2}+\int_{-\infty}^{0} \mathrm{~d} k\left|\widetilde{\psi}_{(2)}(k)\right|^{2}+2 \int_{-\infty}^{0} \mathrm{~d} k \operatorname{Re} \overline{\widetilde{\psi}_{(1)}} \widetilde{\psi}_{(2)} .
$$

The first term simply gives

$$
\int_{-\infty}^{0} \mathrm{~d} k\left|\widetilde{\psi}_{(1)}(k)\right|^{2}=\int_{-\infty}^{0} \mathrm{~d} k\left|\widetilde{\psi}_{T}(k)\right|^{2}
$$

which is a constant term in the expansion. The physical interpretation of this term is obvious. It corresponds to the initially left moving part of the wave packet which reaches the detector in any case, even without switching on the mirror. The second term is the result obtained for the case of only positive incident momenta. The mixed term

$$
2 \int_{-\infty}^{0} \mathrm{~d} k \operatorname{Re} \overline{\widetilde{\psi}_{(1)}} \widetilde{\psi}_{(2)}=2 \frac{\sqrt{2 \pi \alpha}}{\pi^{2}} \int_{-\infty}^{0} \mathrm{~d} k \operatorname{Re}\left\{\overline{\widetilde{\psi}(k)} \mathrm{e}^{\mathrm{i} \alpha k^{2}} \mathscr{P} \int_{-\infty}^{\infty} \mathrm{d} k^{\prime} \frac{1}{k^{\prime}-\sqrt{\alpha} k} f_{\alpha}\left(k^{\prime}\right)\right\}
$$


is going to be evaluated in the following. Since in this term only one Hilbert transform is involved it is not possible to apply the identity (5.38). An explicit evaluation of the Hilbert transforms yields

$$
\begin{aligned}
& 2 \int_{-\infty}^{0} \mathrm{~d} k \operatorname{Re} \overline{\widetilde{\psi}_{(1)}} \widetilde{\psi}_{(2)}= \\
& -2 \sqrt{\frac{2 \alpha}{\pi}} \operatorname{Re}\left\{( 1 + \mathrm { i } ) \left[\psi(0) \int_{-\infty}^{0} \mathrm{~d} k \widetilde{\psi}(k) \frac{\mathrm{C}\left(\sqrt{\frac{2 \alpha}{\pi}} k\right)+\mathrm{iS}\left(\sqrt{\frac{2 \alpha}{\pi}} k\right)}{\sqrt{\alpha} k}\right.\right. \\
& \left.\left.-\alpha \psi^{\prime \prime}(0) \int_{-\infty}^{0} \mathrm{~d} k \overline{\widetilde{\psi}(k)} \frac{\left(\mathrm{C}\left(\sqrt{\frac{2 \alpha}{\pi}} k\right)+\mathrm{iS}\left(\sqrt{\frac{2 \alpha}{\pi}} k\right)\right)-\mathrm{e}^{\mathrm{i} \alpha k^{2}} \sqrt{\frac{2 \alpha}{\pi} k}}{(\sqrt{\alpha} k)^{3}}+\mathcal{O}\left(\alpha^{2}\right)\right]\right\}
\end{aligned}
$$

where $\mathrm{C}(\cdot)$ and $\mathrm{S}(\cdot)$ are the Fresnel cosine and sine functions as defined in appendix C. An expansion of the fractions in this expression then gives

$$
\begin{aligned}
& 2 \int_{-\infty}^{0} \mathrm{~d} k \operatorname{Re} \overline{\widetilde{\psi}_{(1)}} \widetilde{\psi}_{(2)}= \\
& -4 \frac{\sqrt{\alpha}}{\pi} \operatorname{Re}\left\{( 1 + \mathrm { i } ) \left[\psi(0) \int_{-\infty}^{0} \mathrm{~d} k \widetilde{\psi}(k)\left[1+\frac{\mathrm{i} \alpha k^{2}}{3}-\frac{\alpha^{2} k^{4}}{10}+\mathcal{O}\left(\alpha^{3}\right)\right]\right.\right. \\
& -\alpha \psi^{\prime \prime}(0) \int_{-\infty}^{0} \mathrm{~d} k \bar{\psi} \widetilde{\psi}(k)\left[-\frac{2 \mathrm{i}}{3}+\frac{6 \alpha k^{2}}{15}+\mathcal{O}\left(\alpha^{2}\right)\right] \\
& \left.\left.+\alpha^{2} \psi^{(4)}(0) \int_{-\infty}^{0} \mathrm{~d} k \bar{\psi}(k)\left[-\frac{4}{15}+\mathcal{O}(\alpha)\right]+\mathcal{O}\left(\alpha^{3}\right)\right]\right\} \\
& =-4 \frac{\sqrt{\alpha}}{\pi}\left[\operatorname{Re}\left\{(1+\mathrm{i})\left[\psi(0) \int_{-\infty}^{0} \mathrm{~d} k \widetilde{\widetilde{\psi}(k)}\right]\right\}\right. \\
& -\frac{\alpha}{3}\left(\operatorname{Re}\left\{(1-\mathrm{i})\left[\psi(0) \int_{-\infty}^{0} \mathrm{~d} k k^{2} \widetilde{\widetilde{\psi}(k)}\right]\right\}+2 \operatorname{Re}\left\{(1+\mathrm{i})\left[\overline{\psi^{\prime \prime}(0)} \int_{-\infty}^{0} \mathrm{~d} k \widetilde{\psi}(k)\right]\right\}\right) \\
& -\alpha^{2}\left(\frac{2}{5} \operatorname{Re}\left\{(1+\mathrm{i})\left[\psi^{\prime \prime}(0) \int_{-\infty}^{0} \mathrm{~d} k k^{2} \widetilde{\widetilde{\psi}(k)}\right]\right\}+\frac{1}{10} \operatorname{Re}\left\{(1+\mathrm{i})\left[\psi(0) \int_{-\infty}^{0} \mathrm{~d} k k^{4} \widetilde{\psi}(k)\right]\right\}\right. \\
& \left.\left.+\frac{4}{15} \operatorname{Re}\left\{(1-\mathrm{i})\left[\overline{\psi^{(4)}(0)} \int_{-\infty}^{0} \mathrm{~d} k \widetilde{\psi}(k)\right]\right\}\right)+\mathcal{O}\left(\alpha^{3}\right)\right] .
\end{aligned}
$$

There is a simple possibility to check this result. If one shifts the upper integration limit to infinity in Equation (5.52) the result should be unity. The same is true for the integral with $\widetilde{\psi}_{(1)}(k)$ on the right hand side of this equation. Since the integral with $\widetilde{\psi}_{(2)}(k)$ gives the same result when integrating over only positive or only negative $k$ the mixed term integrated over all $k$ should give twice the result for the norm of the reflected wave packet in the case of momentum amplitudes with 
only positive $k$, only with the opposite sign. This is indeed the case for the above result.

In a measurement it would be desirable to be able to distinguish between $\widetilde{\psi}_{(1)}$ and $\widetilde{\psi}_{(2)}$ because only the latter has actually been reflected at the mirror. This is rather simple for the pure term of Equation (5.53). Since it is independent of $k$ it forms a constant background signal which can be extracted by measuring with different values of $\tau$. The interference term of Equation (5.54) is much more problematic because in lowest order it also gives a term proportional to $\sqrt{\tau}$. One might try separate the two terms by measuring the reflected signal for different ranges of $k$. The problem with this is, however, that $\psi_{(1)}$ may have an arbitrary momentum distribution.

\subsection{Numerical analysis of the model}

In order to gain more physical insight into the model, the analytical investigations of the previous section are complemented with some numerical analysis in this section. All the numerical calculations presented in the following use, for convenience, a minimum-uncertainty Gaussian wave packet

$$
\psi_{t}(x)=\sqrt{\frac{\Delta_{x}}{\sqrt{2 \pi}}} \frac{1}{\sqrt{\Delta_{x}^{2}+\mathrm{i} \frac{\hbar t}{2 m}}} \mathrm{e}^{\mathrm{i}\left(k_{0} x-\frac{\hbar k_{0}^{2}}{2 m} t\right)} \mathrm{e}^{-\frac{\left(x-x_{0}-v_{0} t\right)^{2}}{4\left(\Delta_{x}^{2}+\mathrm{i} \frac{\hbar t}{2 m}\right)}}
$$

which is prepared at time $t=0$ at a position $x_{0}$ left of the mirror and with a width $\Delta_{x}$ in position space. This wave packet has in principal also negative momentum components which are, however, negligibly small for numerical purposes. The parameter values given in the following for each figure always correspond to the quantities in the above equation. The corresponding momentum amplitude $\psi_{t}(k)$ can be written in the form

$$
\widetilde{\psi}_{t}(k)=\sqrt{\frac{\Delta_{x}}{\sqrt{2 \pi}}} \mathrm{e}^{-\Delta_{x}^{2} k_{0}^{2}+\mathrm{i} k_{0} x_{0}} \mathrm{e}^{\beta^{2} / \alpha} \mathrm{e}^{-\alpha(k-\beta / \alpha)^{2}},
$$

with $\alpha=\Delta_{x}^{2}+\mathrm{i} \frac{\hbar t}{2 m}$ and $\beta=k_{0} \Delta_{x}^{2}-\mathrm{i} x_{0} / 2$. Therefore, using Equation (C.10) of appendix $\mathrm{C}$, one can perform the Hilbert transform of this function analytically

$$
g_{t}(k)=H\left[\widetilde{\psi}_{t}\right]=-\sqrt{\frac{\Delta_{x}}{\sqrt{2 \pi}}} \mathrm{e}^{-\Delta_{x}^{2} k_{0}^{2}+\mathrm{i} k_{0} x_{0}} \mathrm{e}^{\beta^{2} / \alpha} \mathrm{e}^{-\alpha(k-\beta / \alpha)^{2}} \operatorname{erfi}(\sqrt{\alpha}(k-\beta / \alpha)),
$$

where erfi(.) is the imaginary error function. This can be used to compute the momentum amplitude of the reflected part of the wave function according to Equation (5.18). In Figure 5.2 this has been done for different switch-on times $\tau$ of the mirror. In the calculations parameter values corresponding to the physical situation in the 

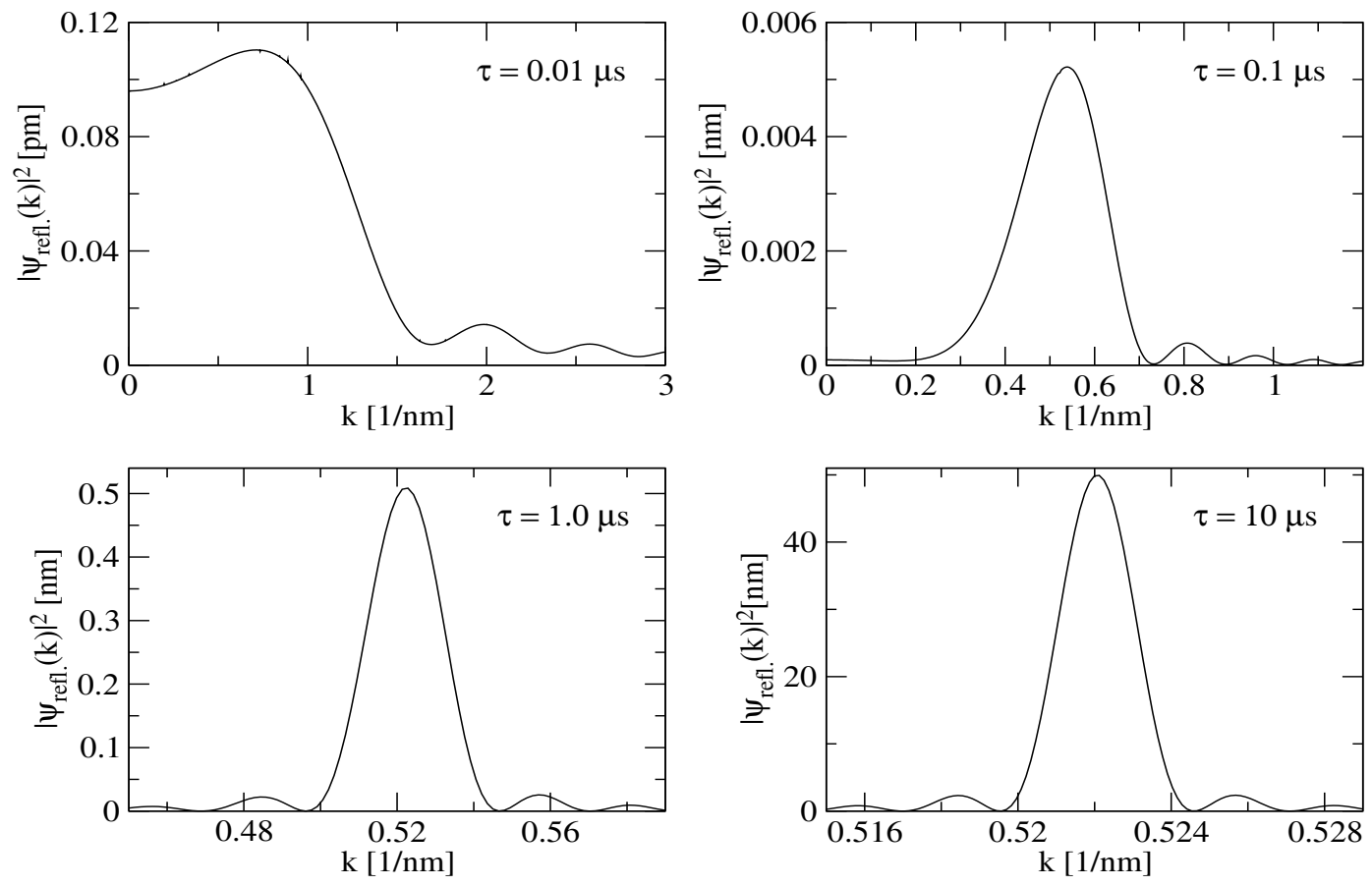

Figure 5.2: Momentum distributions for the reflected part of the wave packet for different switch-on times $\tau$. For very short times the distribution is broad and becomes more and more narrow for longer switch on times.

experiment have been used. The wave packet reaches the measurement pulse at a time $T=50 \mathrm{~ms}$ after the second rebound, which is regarded here as the moment of preparation, with a velocity $v_{0}=25 \mathrm{~cm} / \mathrm{s}$. Note, however, that in the simplified model used here one has free motion instead of free fall in gravity. Thus, the position $x_{0}=-12.5 \mathrm{~mm}$ of preparation is matched such that the wave packet reaches the mirror after the experimental time $T$. Also note, that the wave packet in the experiment has a sinc-shaped form in contrast to the Gaussian used here. One can see in Figure 5.2 that for very small $\tau$ the momentum distribution of the reflected wave packet is much broader than the distribution of the incoming packet with a significant contribution near $k=0$. For increasing $\tau$ the distribution becomes sharper and sharper. However, side-bands remain which originate from the sharp switching processes.

Another important point is the numerical verification of the series expansion for the norm of the reflected part of the wave function given in the last section. For this purpose $N_{\text {refl. }}(\tau)$ may be calculated numerically by integrating the momentum distributions of Figure 5.2 over $k$ and plotting it against $\tau$. This can be compared with the first terms in the expansion of Equation (5.50). In principle, one expects the expansion to give a good approximation to the actual value if the product $\sqrt{\alpha} k$ is small against one, where $k$ ranges over the values for which the momentum distribution gives a noticeable contribution. The former is the case if $\tau<2 m / \hbar k^{2}$. In 


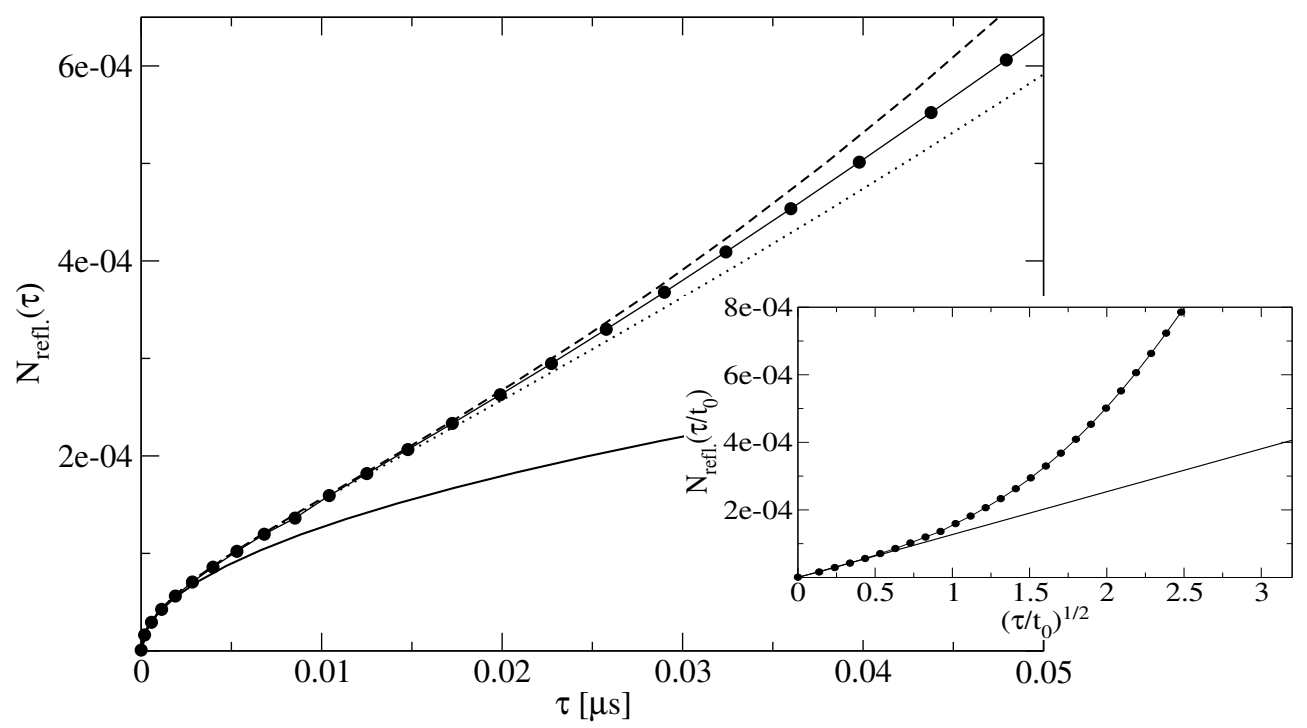

Figure 5.3: Plot of $N_{\text {refl. }}$ against the switch-on time of the mirror for experimental parameter values. Gaussian wave packet prepared at time $t=0$ with mean position $x_{0}=-12 \mathrm{~mm}$, mean velocity $v_{0}=25 \mathrm{~cm} / \mathrm{s}$, initial width $\Delta_{x}=1.6 \mu \mathrm{m}$. Mirror switched on at $T=50 \mathrm{~ms}$. The series expansion (first order: solid line, up to second order: dotted line, up to third order: dashed line) agrees only for very short times $(<0.02 \mu \mathrm{s})$ with the numerical result (thin line with dots). Inset: plot of the numerical and first order result against $\sqrt{\tau / t_{0}}$ with $t_{0}=0.01 \mu \mathrm{s}$.

order to achieve good results for large values of $\tau$ the atom has to be sufficiently slow with a narrow momentum distribution. For a wave packet with the experimental values given above one has the condition $\tau<1.5 \times 10^{-8} \mathrm{~s}$. This is much shorter than the time window used in the experiment. Figure 5.3 shows the numerical result for this case together with the first three orders of the expansion. First of all the $\sqrt{\tau}$-dependence for short switch-on times as predicted by the Equation (5.44) is clearly visible and verified by the inset showing a plot of $N_{\text {refl. }}$ against $\sqrt{\tau}$. The above estimation of the range of validity of the expansion is also confirmed. The first order differs noticeably from the numerical result for $\tau>0.01 \mu \mathrm{s}$. The approximation up to third order gives good results up to $\tau=0.03$. In order to improve the range of validity one may prepare a slower wave packet. This is shown in Figure 5.4 for a wave packet with a mean velocity of $v_{0}=1 \mathrm{~cm} / \mathrm{s}$. One then expects a good agreement of the expansion with the numerical results for $\tau<9.5 \mu \mathrm{s}$.

\subsubsection{Measurement of the spatial distribution of the wave function}

In this section a scheme for the measurement of a spatial distribution with the atomic mirror will be discussed. The basic set-up is the following: An ensemble of atoms 


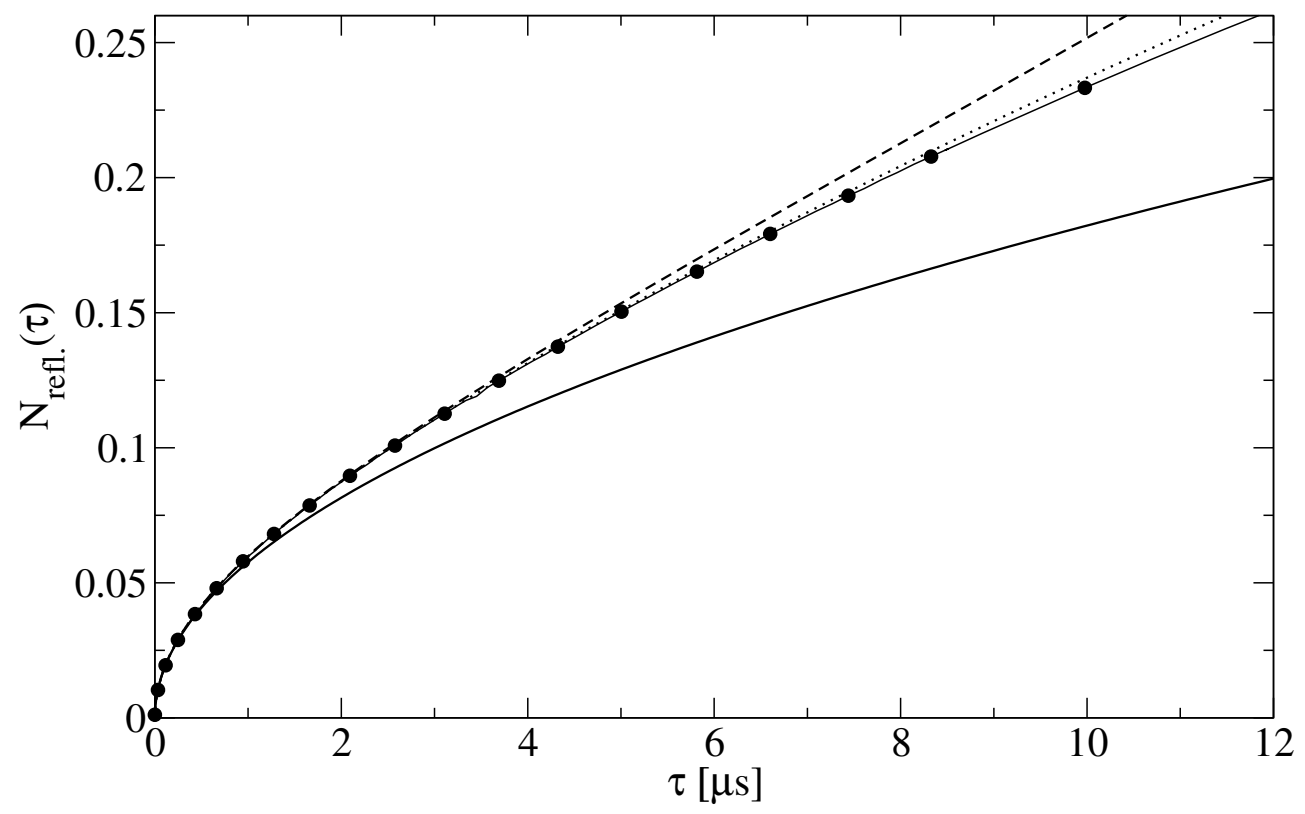

Figure 5.4: Plot of the integrated reflected norm against the switch-on time of the mirror for a slow Gaussian wave packet $v_{0}=1.1 \mathrm{~cm} / \mathrm{s}$ prepared at time $t=0$ with mean position $x_{0}=-0.66 \mu \mathrm{m}$, initial width $\Delta_{x}=0.1 \mu \mathrm{m}$. Mirror switched on at $T=60 \mu \mathrm{s}$. The agreement between the series expansion (up to first order: solid line, up to second order: dashed line, up to third order: dotted line) and the numerical result (thin line with dots) is considerably improved compared to Figure 5.3.

is prepared at some point $x_{0}<0$ in space with a mean velocity $v_{0}>0$. The mirror at $x=0$ is switched on at time $T$ and switched off again at $T+\tau$. Afterwards the number of reflected atoms is counted, for example in a fluorescence measurement. More specifically the reflected atoms pass a laser illuminated region and the number of spontaneously emitted photons is counted by a photon detector. The experiment is repeated and the position of the mirror is changed whereas the instant $T=T_{0}$ of switching on the mirror is kept fixed. There are two different possibilities of moving the mirror. One alternative is to move the prism (and with it the whole set-up for the mirror) mechanically. The other would be to change the laser intensity. In this way the effective reversal point of the atoms is moved. The second technique, however, is limited by the range of laser intensities available.

The distribution which can be measured by the set-up described above will be referred to as $\Lambda_{\text {mirr. }}\left(x, T_{0}\right)$ in the following. For each position $x$ it is given by the norm of the wave packet reflected from the mirror positioned at $x$ and switched-on at $T_{0}$. Due to the finite time window $\tau$ it is more accurate to refer to the point in time in the middle of the time window as the moment of reflection. One therefore has

$$
\Lambda_{\text {mirr. }}\left(x, T_{0}+\tau / 2\right)=N_{T_{0}}^{\text {refl. }}(\tau, x) .
$$




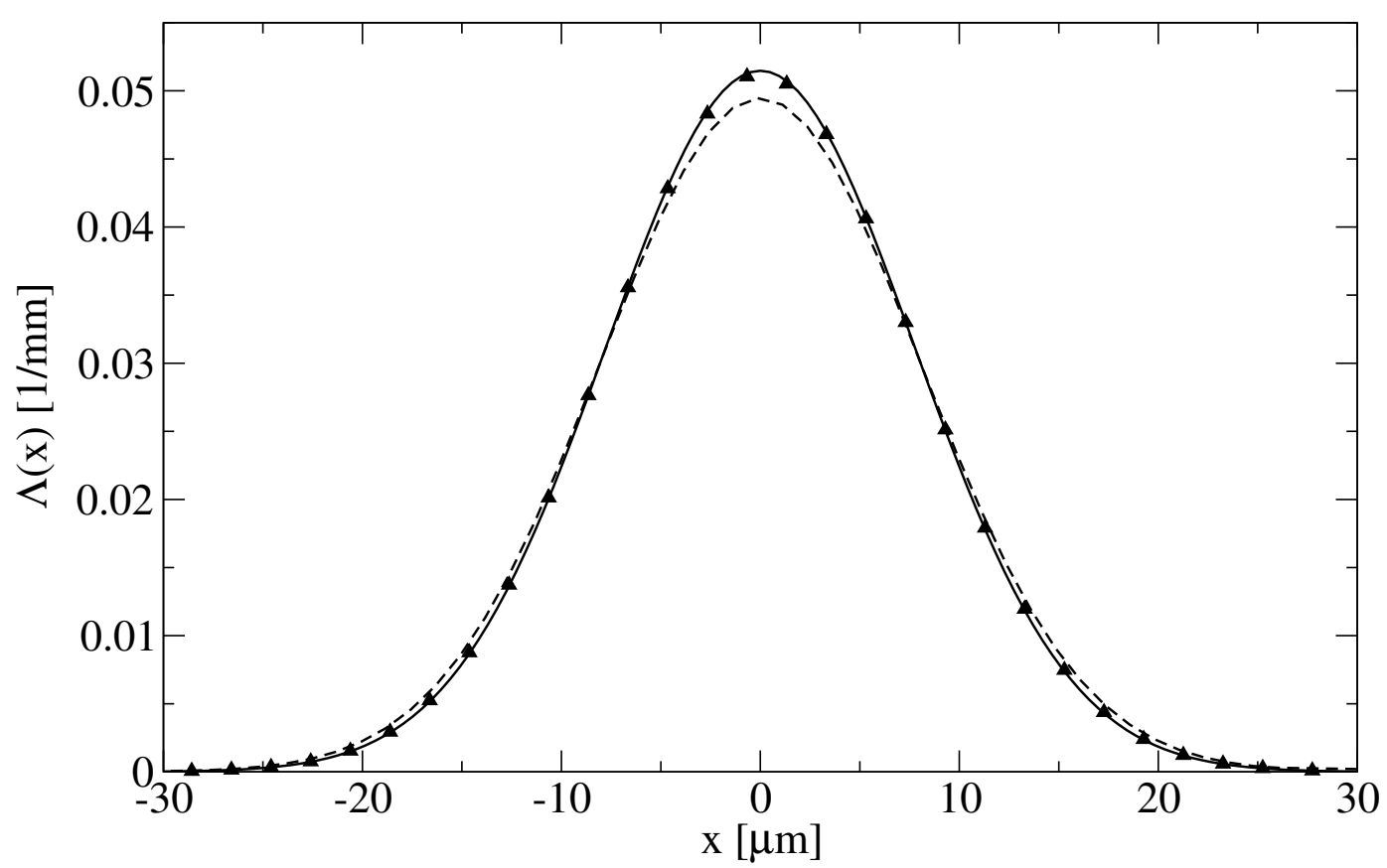

Figure 5.5: Mod squared of the wave function (solid line) and reconstruction for switch-on times $\tau=10 \mu \mathrm{s}$ (dots) and $\tau=30 \mu \mathrm{s}$ (dashed line) for experimental parameter values (see Figure 5.3) at time $T=50 \mathrm{~ms}$.

According to Equation (5.44) the normalized distribution for sufficiently small $\tau$ is given by

$$
\Lambda\left(x, T_{0}\right)=\left|\psi_{T_{0}}(x)\right|^{2} .
$$

The present scheme thus allows to obtain the probability density of the wave function. This gives therefore a realistic possibility of measuring this otherwise not easily accessible quantity and is the main result of this part of the work. Furthermore, it can be regarded as a step towards the quantum state reconstruction of the translational degrees of freedom of a laser cooled atom.

In the field of quantum state reconstruction or estimation one tries to infer the complete information of a quantum state from a series of repeated measurements of this state [53]. This has been very successfully achieved for a number of physical systems, for instance the quantum state of light in a cavity [54] or for the motional and the internal states of trapped ions [55]. Especially the latter has become a standard technique in quantum information processing $[57,58]$. The motional state of unbound atoms is much more difficult to measure. The Wigner function of the transversal state of motion of a supersonic beam of helium has been measured in the group of Mlynek $[60,59]$. This was done in a diffraction experiment with a microfabricated double-slit structure. In such an experiment, however, it is known that the van-der-Waals interaction between the atoms and the grating has a significant influence on the state of the atoms [98]. A somewhat similar experiment has also 


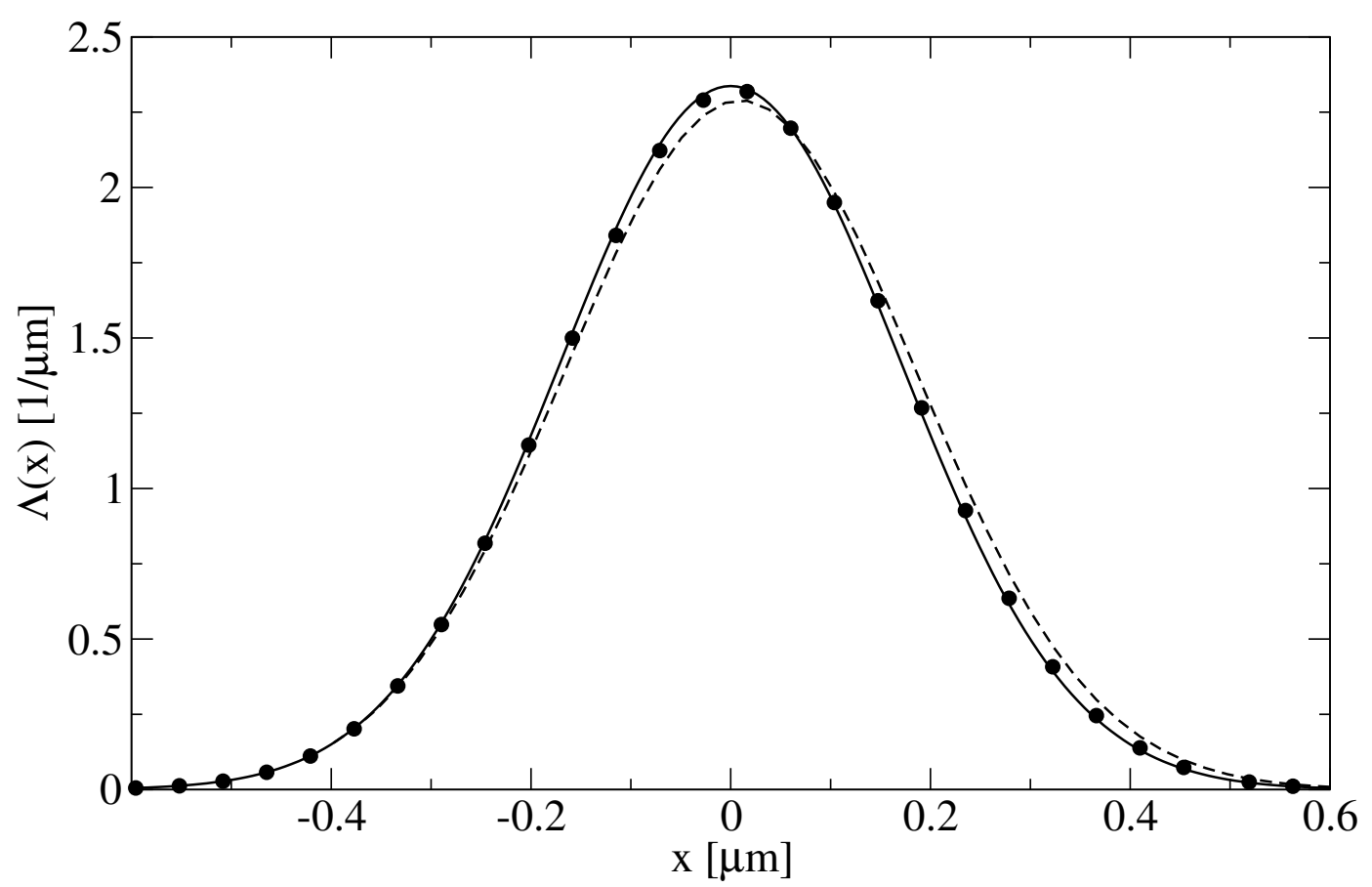

Figure 5.6: Norm squared of the wave function (solid line) and reconstruction for switch-on time $\tau=1 \mu \mathrm{s}$ (dots) and $\tau=5 \mu \mathrm{s}$ for the wave packet of Figure 5.4 at time $T=60 \mu \mathrm{s}$.

been done for a beam of neutrons [99]. In an experiment with a supersonic beam of natrium atoms in the group of Pritchard the longitudinal state of the atoms was analyzed, also by interferometric methods [100, 101, 61, 102, 62, 103]. For theoretical proposals for the measurement of the quantum state of motion of atomic beams see Reference [104] and references therein.

Such a state reconstruction has not yet been achieved for laser cooled atoms. A main problem is the influence of the measurement apparatus on the motional state of the atom. For example, an interferometric measurement using a standing light field as diffraction grating leads to partial reflection of the atomic wave function [105]. The method presented here circumvents this problem by using reflection for which the reduction of losses is easier to achieve. Moreover it is experimentally feasible since it only requires a minor change in set-up of an experiment which has already been performed. However, it only allows to measure the modulus squared of the wave function. The information about the phase of the wave function is lost.

In Figures 5.5 and 5.6 two examples of the reconstruction of the probability density of the wave function are shown. The first shows the numerical result for a wave packet with the experimental parameter values as used for figure 5.7. Surprisingly, a good result is achieved for time windows up to $\tau=10 \mu \mathrm{s}$ although the result for the expansion in $\tau$ suggests that a close approximation to the probability density should only be possible for much shorter values of $\tau$. Apparently, possible devia- 
tions are compensated by the normalization process. Also, the narrow momentum distribution of this wave packet may have a positive influence on the accuracy of the reconstruction. For larger switch-on times deviations occur, as shown in the graph for $\tau=30 \mu \mathrm{s}$. The second plot shows the results for a slow atom with parameter values as in Figure 5.8. In this case deviations from the theoretical curve start to develop for switch-on times larger than $\tau=1 \mu \mathrm{s}$. This is in agreement with the result of Figure 5.4.

\subsubsection{Measurement of temporal distributions}

In this section a measurement scheme will be described and analyzed numerically which in the case of classical mechanics yields the arrival-time distribution of an atomic ensemble. Due to the difficulties outlined in Section 4.3 concerning the quantum version of this distribution it will be a major question here whether the scheme to be described in the following can also quantum mechanically be ascribed to an arrival-time measurement. The scheme is basically the same as described in the last section. Only now, the experiment is repeated with the position $x=0$ of the mirror kept fixed and the switch-on time $T$ varied.

For a cloud of classical particles this set-up would yield the classical arrival-time, as will be shown now. In the classical case one has to calculate the fraction of reflected particles if at $t=0$ the phase-space distribution function of the particles is given by $\rho_{0}(x, p)$ and the mirror at position $x_{\mathrm{A}}$ is switched on in the interval $[T, T+\tau]$. It is supposed that $\rho_{0}(x, p)=0$ for $p \leq 0$. Using the trajectory equation for free motion one finds that all particles which are located in the interval $\left[x_{1}, x_{2}\right]$ with

$$
x_{1}=x_{\mathrm{A}}-\frac{p(T+\tau)}{m}, \quad x_{2}=x_{\mathrm{A}}-\frac{p T}{m}
$$

at time $t$ are reflected. Thus, the fraction of reflected particles is given by

$$
n_{x_{\mathrm{A}}}(\tau)=\int_{0}^{\infty} \mathrm{d} p \int_{x_{1}}^{x_{2}} \mathrm{~d} x \rho_{0}(x, p)=-\int_{0}^{\infty} \mathrm{d} p \int_{x_{\mathrm{A}}-\frac{p T}{m}}^{x_{\mathrm{A}}-\frac{p(T+\tau)}{m}} \mathrm{~d} x \rho_{0}(x, p) .
$$

Now the result for short switch-on times $\tau$ is to be evaluated. For $\tau=0$ one has $n_{x_{\mathrm{A}}}(\tau=0)=0$. The term proportional to $\tau$ yields

$$
\begin{aligned}
\left.\frac{\mathrm{d}}{\mathrm{d} \tau} n_{x_{\mathrm{A}}}(\tau)\right|_{\tau=0} & =\int_{0}^{\infty} \mathrm{d} p \frac{p}{m} \rho_{0}\left(x_{\mathrm{A}}-\frac{p}{m} T, p\right) \\
& =\int_{0}^{\infty} \mathrm{d} p \int_{-\infty}^{\infty} \mathrm{d} x \rho_{T}(x, p) \delta\left(x-x_{\mathrm{A}}\right)=J^{\mathrm{cl}}\left(T, x_{\mathrm{A}}\right) .
\end{aligned}
$$

Therefore for short times $\tau$ the number of reflected particles is proportional to the classical probability flux which has been identified in Section 4.3 with the classical arrival-time distribution. 
It is therefore natural to ask the question which quantity is obtained by this setup according to quantum mechanics. According to Equation (5.44) and assuming perfect reflection the resulting distribution is given by

$$
\Pi_{\exp }(T, 0)=2 \sqrt{\frac{\hbar \tau}{m \pi}}\left|\psi_{T}(0)\right|^{2}
$$

if the mirror has been switched-on for sufficiently short times $\tau$. As before it is more accurate to use $T+\tau / 2$ instead of $T$. This distribution is not normalized to one. The normalization can be done by deviding by the integral $\int \mathrm{d} T \Pi_{\exp }(T)$. This has the advantage that the resulting distribution is free of effects of a less than perfect detector efficiency. The normalized distribution is identical to the semi-classical arrival-time distribution which has been derived in Section 4.3.2 as an approximation to Kijowski's axiomatic distribution for wave packets with a sharply peaked momentum distribution.

The measurement scheme described above is therefore not capable of measuring what is regarded as a true quantum mechanical arrival-time distribution but rather gives an approximation to such a distribution. Consequently, the labeling "arrivaltime" in the diffraction curves measured in Reference [49] should be used with some caution.

Figures 5.7,5.8, and 5.9 show examples for the scheme described above for different wave packets. In Figure 5.8 the result for a wave packet with the values from the experiment is shown. Since in this case the momentum spread is very small Kijowski's distribution $\Pi_{\mathrm{K}}(0, t)$, the flux $J(0, t)$, and the semi-classical distribution $|\psi(0, t)|^{2} / \int \mathrm{d} t$. give the same graph. As for the corresponding case in the last section, the distribution obtained from the model for $\tau=10 \mu \mathrm{s}$ is in very good agreement with the theoretical curves although the expansion in $\tau$ is not valid for such large switch-on times. For $\tau=30 \mu$ s deviations from the theoretical curve start to form which seem to originate from the fact that the time window is too coarse to reproduce the details of the distribution.

Differences between the different arrival-time distributions are expected for wave packets for which $\Delta v / v_{0}$ is sufficiently large, where $\Delta v$ is the velocity spread and $v_{0}$ is the mean velocity of the packet. Such a situation is shown in figure 5.8 using a wave packet with $v_{0}=1.1 \mathrm{~cm} / \mathrm{s}$ and $\Delta_{x}=0.11 \mu \mathrm{m}$. The graph for the reconstructed distribution with $\tau=0.1 \mu \mathrm{s}$ is in good agreement with the normalized probability density. This shows that indeed neither Kijowski's distribution nor the flux is measured by the present set-up. For $\tau=5 \mu$ s noticeable deviations occur which in this case is in good agreement with the expectations from the result for the expansion in $\tau$.

Another good example for a wave packet for which the different theoretical arrival-time distributions are distinguishable is a coherent superposition of two Gaussians with different mean velocity. Such an example is shown in figure 5.9. The two Gaussian states are prepared such that they superimpose at $x=0$. The difference of the mean velocities is of the order of the velocities themselves. The additional 


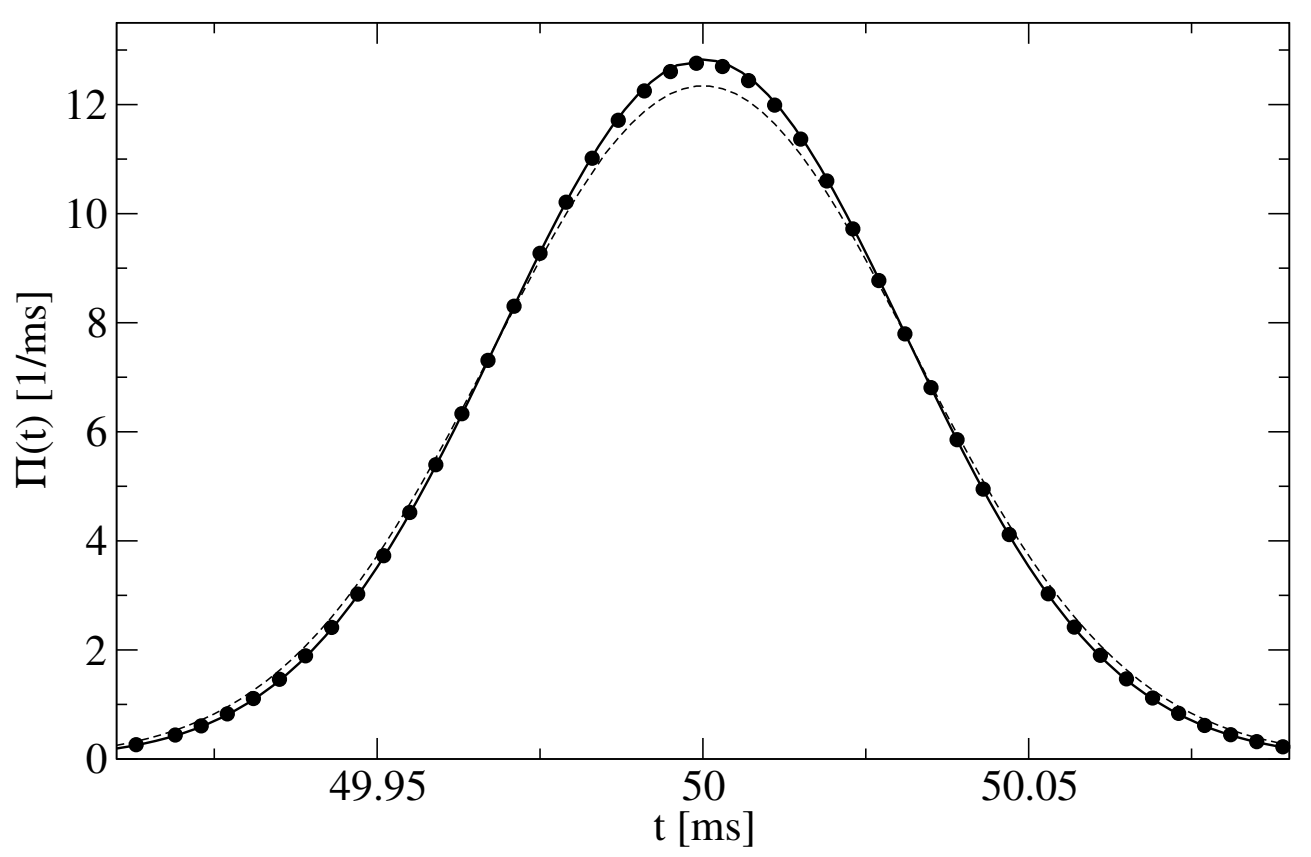

Figure 5.7: Theoretical time-of-arrival distributions and results from the model for a wave packet with experimental parameter values (see Figure 5.3). The different theoretical arrival-time distributions (straight line) are indistinguishable in this case. Reconstructions for time windows $\tau=10 \mu \mathrm{s}$ (dots) and $\tau=30 \mu \mathrm{s}$ (dashed line) are shown.

problem for the measurement of this wave packet is that it has a finer structure due to the inference. Thus, one needs a short enough time window in order to resolve these structures.

Recently, a quantum optical model for the measurement of the time of arrival has been proposed in which the detection time of the first fluorescence photon of a two-level atom impinging on a laser illuminated region is taken as the arrival-time of the atom [64]. This first photon distribution could be linked to ideal quantities like Kijowski's distribution or the quantum mechanical flux [65]. For the experimental realization of such a quantum optical model a major technical challenge is that it is necessary to achieve a laser profile which is sharp compared to the width of the wave packet in order have a well defined arrival point. At the same time the atoms for which the arrival-time is to be observed have to be sufficiently slow so that quantum effects can be observed. The scheme presented here has the advantage of experimental feasibility since a corresponding experiment has already been performed. However, the connection to the named ideal quantities is not established so far. This might be a possibility for further research. 


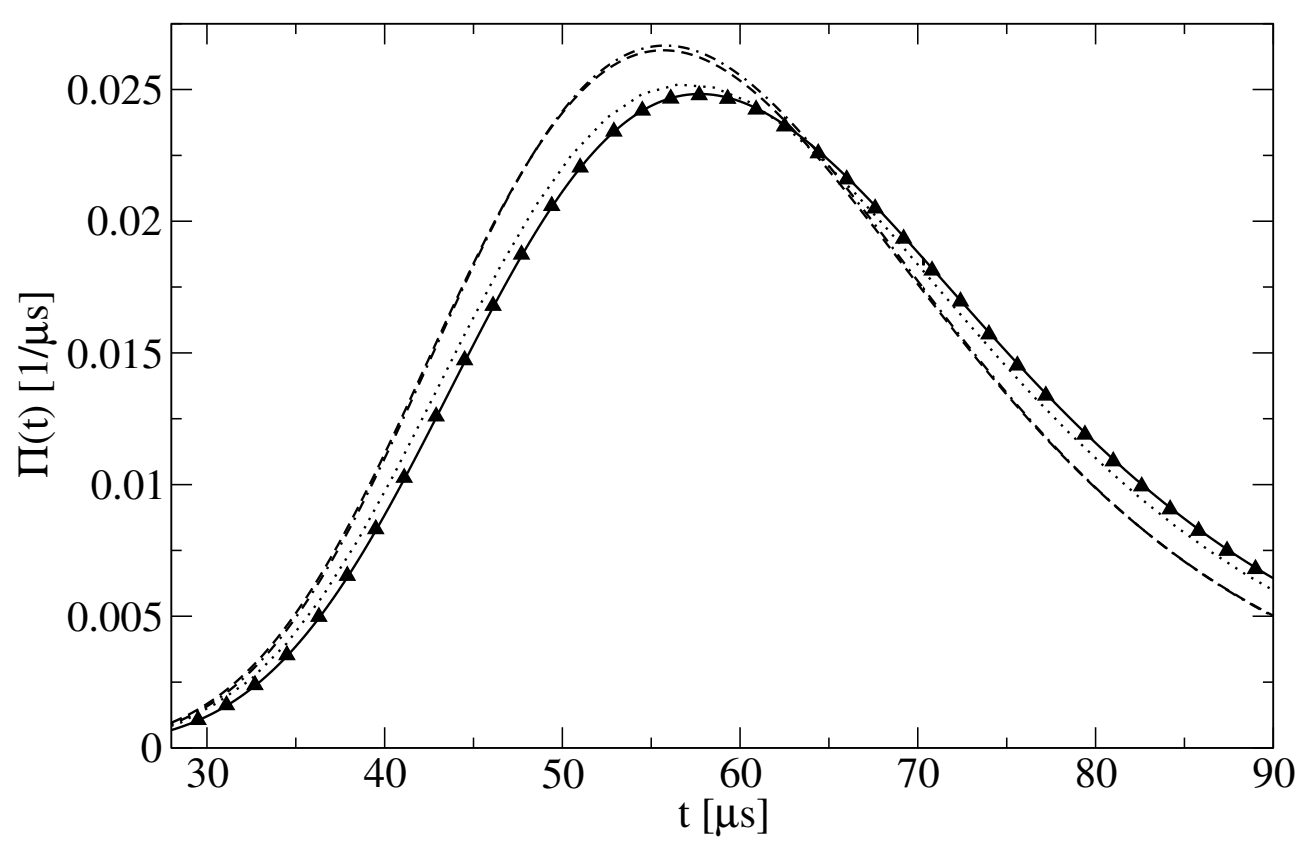

Figure 5.8: Theoretical time-of-arrival distributions and results from the model for a slow wave packet with relatively broad momentum distribution. Minimum uncertainty Gaussian wave packet prepared at $t=0$ with parameter values as in Figure 5.4. The straight line represents the normalized distribution $\Pi_{s c}(t)$, the dashed line is Kijowski's distribution $\Pi_{K}(t)$, and the almost identical dash-dotted line gives the flux $J(0, t)$. Reconstruction for time windows $\tau=0.1 \mu \mathrm{s}$ (triangles) and $\tau=5 \mu \mathrm{s}$ (dotted line) are shown.

\subsubsection{Reflected and transmitted wave packet in position space}

In the case of the measurements for fixed position $x_{0}$ and shifted time $T$ one may ask the question if it is possible to perform multiple measurements at different times in the same measurement cycle. In other words, one might try to switch on the mirror more than once during the passage of the wave packet. In this way one could gain more or less the same data in one measurement instead of having to repeat the experiment for each data point. There are two potential problems with this approach. First, the wave packet which is to be measured might be changed by the mirror, so that the second measurement would be disturbed by the first one. Second, if the time between two subsequent measurement pulses is too short the reflected wave packets of these pulses could overlap and therefore not be distinguished anymore.

In order to investigate these questions the time development of the wave function in position space after switching off the mirror has to be calculated. This can be 


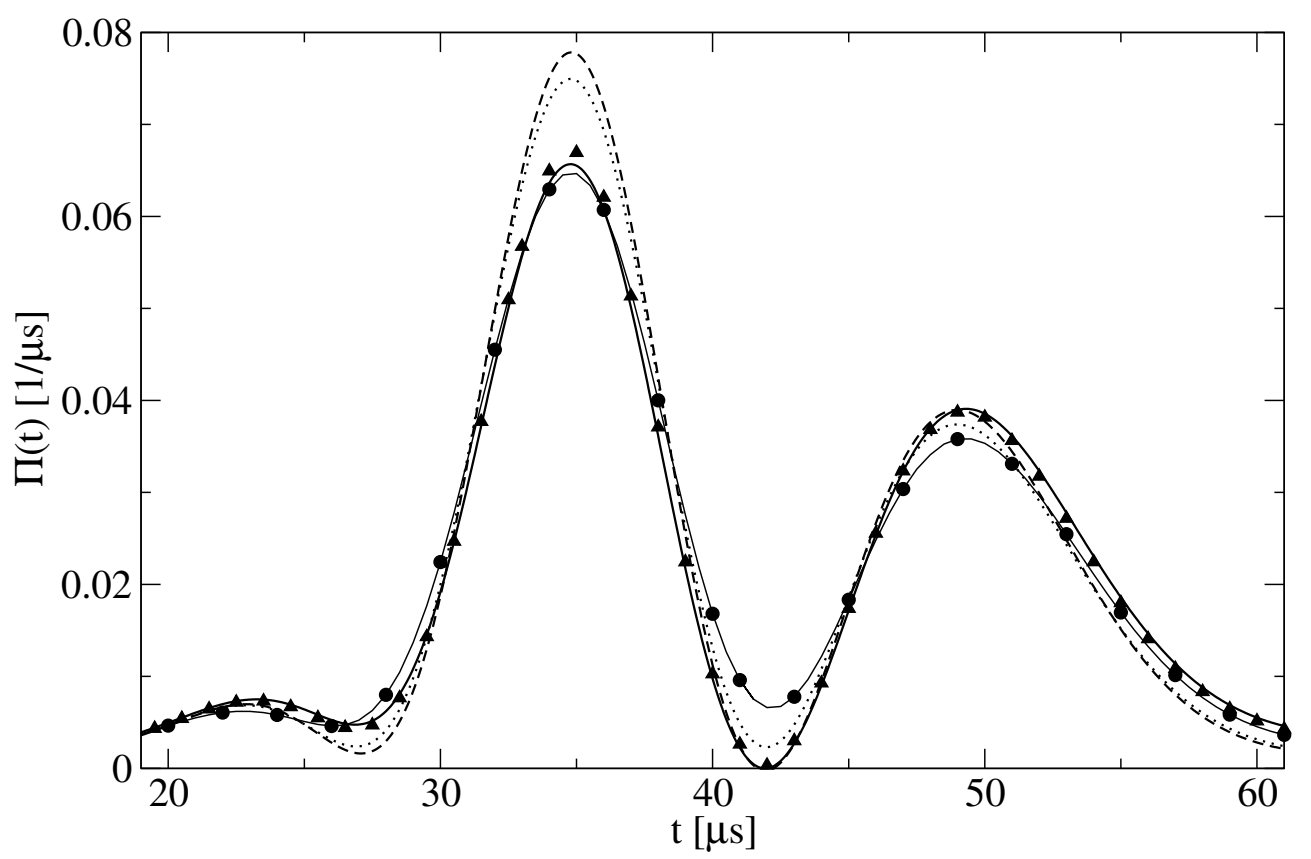

Figure 5.9: Time-of-arrival distributions for coherent superposition of two Gaussians prepared at $t=0$ with $x_{1}=-0.8 \mu \mathrm{m}, x_{2}=-0.4 \mu \mathrm{m}, v_{1}=2.2 \mathrm{~cm} / \mathrm{s}$, $v_{2}=1.1 \mathrm{~cm} / \mathrm{s}$, and $\Delta x=0.1 \mu \mathrm{m}$. Density $\Pi_{s c}(t)$ (solid line), flux $J(0, t)$ (dashed line), and Kijowski's distribution $\Pi_{K}(t)$ (dotted line) differ in this case. The reconstruction for $\tau=1 \mu \mathrm{s}$ (triangles) is still a good approximation to the density whereas for $\tau=5 \mu \mathrm{s}$ (dots with thin solid line) differences appear.

done according to equation (5.29) by the formula

$$
\begin{aligned}
\psi_{T+\tau+t}(x)=G_{t}^{\mathrm{F}} *[ & \theta\left(G_{\tau}^{\mathrm{F}} *\left(\theta \psi_{T}\right)\right)-\theta\left(G_{\tau}^{\mathrm{F}} * \pi\left(\theta \psi_{T}\right)\right) \\
& \left.+\theta_{-}\left(G_{\tau}^{\mathrm{F}} *\left(\theta_{-} \psi_{T}\right)\right)-\theta_{-}\left(G_{\tau}^{\mathrm{F}} * \pi\left(\theta_{-} \psi_{T}\right)\right)\right](x) .
\end{aligned}
$$

As before, $T$ is the switch-on time, $\tau$ is the duration of the time slit, and $t$ is the elapsed time after the mirror has been switched off. If the wave packet at time $T$ is given by the Gaussian of Equation (5.57) the evolution up to time $T+\tau$ can be calculated analytically. The result is lengthy, so it will not be given here. Basically it consists of a linear combination of products of Gaussians and error functions. The second convolution for the time evolution after the switch-off time has to be performed numerically. Figure 5.10 shows $\left|\psi_{\text {refl. }}(x, t)\right|^{2}$ for different times $t$ after reflection from the mirror with a pulse length of $\tau=30 \mu \mathrm{s}$. Here $t$ denotes the time which has passed after the mirror has been switched off. All other parameters are chosen as in the previous example. Shortly after reflection the wave packet has a width corresponding to $v_{0} \tau$ and has a form which resembles the diffraction image of a single slit in the Fresnel limit of classical optics. For longer times the wave packet broadens and reshapes. Eventually, at time $t=50 \mathrm{~ms}$, it has a sinc-function 

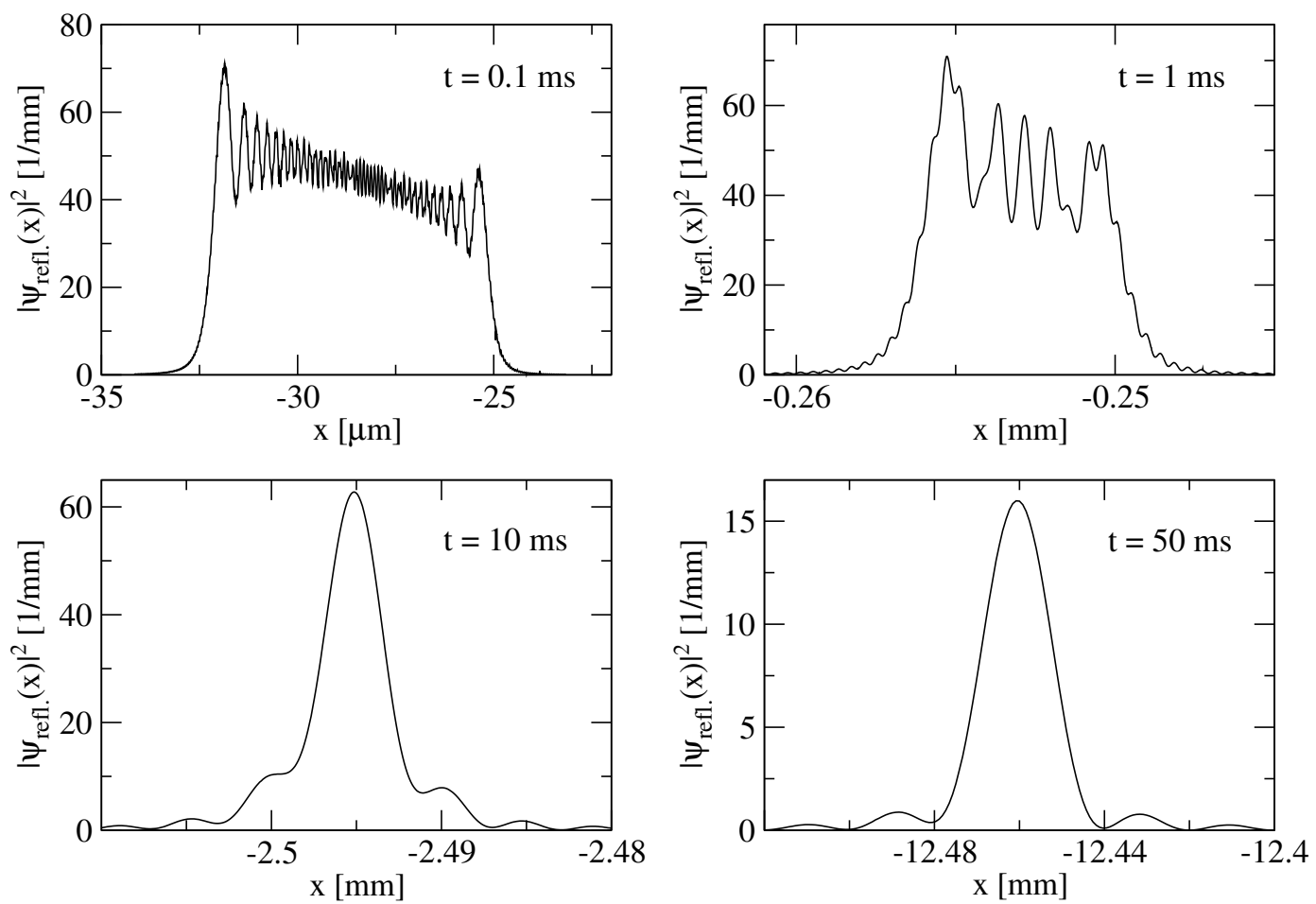

Figure 5.10: Reflected part of the wave packet for the case of a Gaussian wave packet with experimental parameter values at different times $t$ after the switch-off time of the mirror.

form resembling the Fraunhofer limit of classical optics. Moreover, the shape and the width $\Delta_{x}$ are similar to the signal measured for a pulse length of $\tau=30 \mu \mathrm{s}$ in the experiment. One has to keep in mind here that the measured graph shows the wave function after the second rebound whereas here, the wave function reflected by the measurement pulse has been calculated Nevertheless, this can be seen as a confirmation of the validity of the model used here and the approximations connected with it.

In the context of the question posed at the beginning of this section one sees that the determination of the norm of the reflected wave packet has to be done shortly after the reflection in order to be able to resolve subsequent reflected wave packets. In the example shown in Figure 5.10 the wave packet at time $t=1 \mathrm{~ms}$ still has almost the original width of about $7.5 \mu \mathrm{m}$. At time $t=50 \mathrm{~ms}$ it already has a width of about $25 \mu \mathrm{m}$. This corresponds to a time resolution of about $\Delta_{x} / v_{0}=0.1 \mathrm{~ms}$ in contrast to the original $30 \mu \mathrm{s}$ time window of the mirror.

However, the more severe problem is indeed the disturbance of the original wave function by the reflection process, as can be seen in Figure 5.11. Due to the sharp switching process the transmitted wave packet shows strong oscillations with a period much shorter than the width of the wave packet itself. One might hope that for 

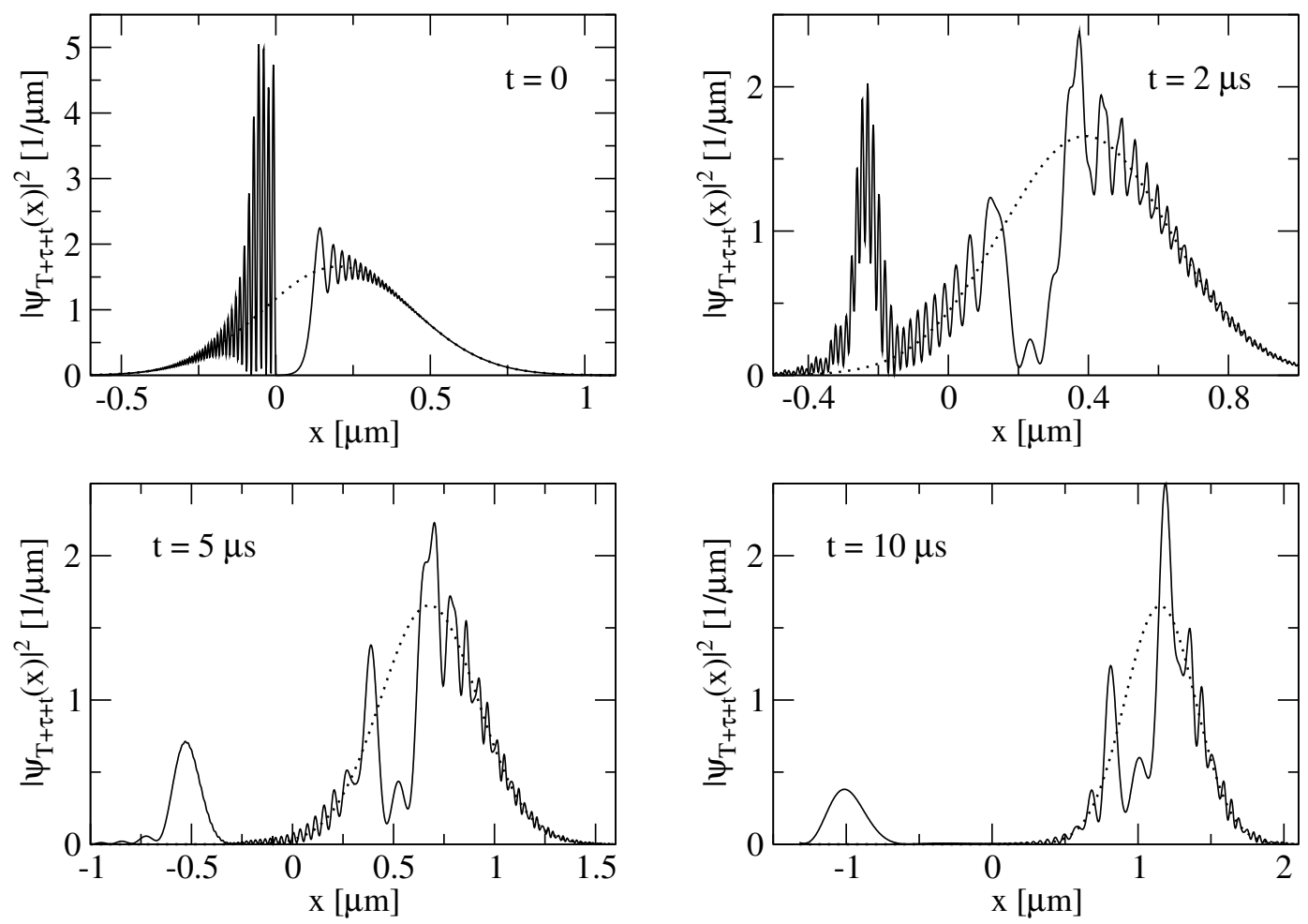

Figure 5.11: Wave packet (solid line) in position space after switching of the mirror $\left(v_{0}=9.5 \mathrm{~cm} / \mathrm{s}, \Delta_{x}=0.24 \mu \mathrm{m}, x_{0}=-1.3 \mu \mathrm{m}, \tau=1 \mu \mathrm{s}, \mathrm{T}=15 \mu \mathrm{s}\right)$. The dotted line shows the freely evolved wave packet.

a reflection time window which cuts out a slice of the wave function that is larger than the period of these oscillations the effects may average out. But this would need a more detailed analysis. Another way around this problem is to use a smoother switching. This may reduce the oscillations. Such a reduction of oscillations by using smoother cuts has been seen in the investigation of a related problem [106].

\subsection{D Model: Tomography of the wave packet}

So far, only a one-dimensional model of the atomic mirror experiment has been considered. In this section it will be shown that there is a straight forward generalization of this model to three dimensions. This, in principal, allows the three dimensional measurement of the probability density of an atom by means of tomographic methods.

In the following the reflection of a three dimensional wave packet, incident from the negative $x$-axis, from plane mirror situated at the position $x=0$ is considered. As before the mirror is modeled by the Dirichlet boundary condition $\psi_{t}(x=0, y, z)=$ 0 . In order to calculate the momentum amplitude of the reflected part of the wave 
packet on can proceed analogously to the Green's function method used in the onedimensional problem. The Green's function for a freely moving atom is given by

$$
G_{t}^{\mathrm{F}}(\boldsymbol{x})=\left(\frac{m}{2 \mathrm{i} \pi \hbar t}\right)^{3 / 2} \mathrm{e}^{-\frac{m}{2 \mathrm{i} \hbar t} \boldsymbol{x}^{2}} .
$$

With the use of the three-dimensional convolution formula

$$
f * g=\int_{-\infty}^{\infty} \mathrm{d}^{3} \boldsymbol{x}^{\prime} f\left(\boldsymbol{x}-\boldsymbol{x}^{\prime}\right) g\left(\boldsymbol{x}^{\prime}\right)
$$

the momentum amplitude at switch-off time $t=T+\tau$ again can again be written as

$$
\begin{aligned}
\psi_{T+\tau}(\boldsymbol{x})= & \theta^{x}\left(G_{\tau}^{\mathrm{F}} *\left(\theta^{x} \psi_{T}\right)\right)(\boldsymbol{x})-\theta^{x}\left(G_{\tau}^{\mathrm{F}} * \pi_{x}\left(\theta^{x} \psi_{T}\right)\right)(\boldsymbol{x}) \\
& +\theta_{-}^{x}\left(G_{\tau}^{\mathrm{F}} *\left(\theta_{-}^{x} \psi_{T}\right)\right)(\boldsymbol{x})-\theta_{-}^{x}\left(G_{\tau}^{\mathrm{F}} * \pi_{x}\left(\theta_{-}^{x} \psi_{T}\right)\right)(\boldsymbol{x}) .
\end{aligned}
$$

Here $\theta^{x}=\theta^{x}(\boldsymbol{x})$ is defined as being equal to 1 for $x>0$ and equal to 0 elsewhere. Accordingly, $\theta_{-}^{x}=\theta^{x}(-x, y, z)$. The operation $\pi_{x}$ stands for change of sign of the $x$-coordinate. Using the Fourier space identities

$$
\widetilde{f * g}=(2 \pi)^{3 / 2} \widetilde{f} \widetilde{g} \quad \text { and } \quad \widetilde{f} * \widetilde{g}=(2 \pi)^{3 / 2} \widetilde{f g}
$$

the momentum amplitude at switch-off time $t=T+\tau$ can again be written as

$$
\begin{aligned}
\widetilde{\psi}_{T+\tau}(\boldsymbol{k})=\widetilde{\psi}_{T+\tau}^{\mathrm{L}}(\boldsymbol{k}) & +\widetilde{\psi}_{T+\tau}^{\mathrm{R}}(\boldsymbol{k}) \\
=\frac{1}{(2 \pi)^{3 / 2}} & {\left[\widetilde{\theta}^{x} *\left(\widetilde{G}_{\tau}^{\mathrm{F}}\left(\widetilde{\theta}^{x} * \widetilde{\psi}_{T}\right)\right)(\boldsymbol{k})-\widetilde{\theta}^{x} *\left(\widetilde{G}_{\tau}^{\mathrm{F}} \pi_{k_{x}}\left(\widetilde{\theta}^{x} * \widetilde{\psi}_{T}\right)\right)(\boldsymbol{k})\right.} \\
& \left.\quad+\widetilde{\theta}_{-}^{x} *\left(\widetilde{G}_{\tau}^{\mathrm{F}}\left(\widetilde{\theta}_{-}^{x} * \widetilde{\psi}_{T}\right)\right)(\boldsymbol{k})-\widetilde{\theta}_{-}^{x} *\left(\widetilde{G}_{\tau}^{\mathrm{F}} \pi_{k_{x}}\left(\widetilde{\theta}_{-}^{x} * \widetilde{\psi}_{T}\right)\right)(\boldsymbol{k})\right] .
\end{aligned}
$$

The Fourier transforms involved in this expression are

$$
\begin{aligned}
\widetilde{G}_{\tau}^{\mathrm{F}}(\boldsymbol{k}) & =\frac{1}{(2 \pi)^{3 / 2}} \mathrm{e}^{-\mathrm{i} \frac{\hbar \boldsymbol{k}^{2}}{2 m} \tau}, \\
\widetilde{\theta}^{x}(\boldsymbol{k}) & =\sqrt{2 \pi} \frac{1}{\mathrm{i} k_{x}+\epsilon} \delta\left(k_{y}\right) \delta\left(k_{z}\right), \quad \widetilde{\theta}_{-}^{x}(\boldsymbol{k})=-\sqrt{2 \pi} \frac{1}{\mathrm{i} k_{x}-\epsilon} \delta\left(k_{y}\right) \delta\left(k_{z}\right) .
\end{aligned}
$$

Thus the explicit form of the momentum amplitude for positive values of $x$ is given by

$$
\begin{gathered}
\widetilde{\psi}_{T+\tau}^{\mathrm{R}}(\boldsymbol{k})=\int_{-\infty}^{\infty} \mathrm{d}^{3} \boldsymbol{k}^{\prime} \widetilde{\theta}\left(\boldsymbol{k}-\boldsymbol{k}^{\prime}\right) G_{\tau}^{\mathrm{F}}\left(\boldsymbol{k}^{\prime}\right) \int_{-\infty}^{\infty} \mathrm{d}^{3} \boldsymbol{k}^{\prime \prime}\left[\widetilde{\theta}\left(\boldsymbol{k}^{\prime}-\boldsymbol{k}^{\prime \prime}\right)+\pi_{k_{x}^{\prime}} \widetilde{\theta}\left(\boldsymbol{k}^{\prime}-\boldsymbol{k}^{\prime \prime}\right)\right] \widetilde{\psi}_{T}\left(\boldsymbol{k}^{\prime \prime}\right) \\
\quad=\frac{\mathrm{e}^{-\mathrm{i} \alpha\left(k_{y}^{2}+k_{z}^{2}\right)}}{4 \pi^{2}} \int_{-\infty}^{\infty} \mathrm{d} k_{x}^{\prime}\left[\frac{1}{\mathrm{i}\left(k_{x}-k_{x}^{\prime}\right)+\epsilon}-\frac{1}{\mathrm{i}\left(k_{x}+k_{x}^{\prime}\right)+\epsilon}\right] \mathrm{e}^{-\mathrm{i} \alpha k_{x}^{2}} \int_{-\infty}^{\infty} \mathrm{d} k_{x}^{\prime \prime} \frac{\widetilde{\psi}_{T}\left(k_{x}^{\prime \prime}, k_{y}, k_{z}\right)}{\mathrm{i}\left(k_{x}^{\prime}-k_{x}^{\prime \prime}\right)+\epsilon}
\end{gathered}
$$


and correspondingly for negative values of $x$ by

$$
\begin{aligned}
& \widetilde{\psi}_{T+\tau}^{\mathrm{L}}(\boldsymbol{k})= \\
& \frac{\mathrm{e}^{-\mathrm{i} \alpha\left(k_{y}^{2}+k_{z}^{2}\right)}}{4 \pi^{2}} \int_{-\infty}^{\infty} \mathrm{d} k_{x}^{\prime}\left[\frac{1}{\mathrm{i}\left(k_{x}-k_{x}^{\prime}\right)-\epsilon}-\frac{1}{\mathrm{i}\left(k_{x}+k_{x}^{\prime}\right)-\epsilon}\right] \mathrm{e}^{-\mathrm{i} \alpha k_{x}^{2}} \int_{-\infty}^{\infty} \mathrm{d} k_{x}^{\prime \prime} \frac{\widetilde{\psi}_{T}\left(k_{x}^{\prime \prime}, k_{y}, k_{z}\right)}{\mathrm{i}\left(k_{x}^{\prime}-k_{x}^{\prime \prime}\right)-\epsilon} .
\end{aligned}
$$

So, after the insertion of delta functions and principal value terms according to the identities (5.35), the momentum amplitude at switch-off can be written as

$$
\begin{aligned}
& \widetilde{\psi}_{T+\tau}(\boldsymbol{k})=\widetilde{\psi}_{T}(\boldsymbol{k}) \mathrm{e}^{-\mathrm{i} \alpha \boldsymbol{k}^{2}}
\end{aligned}
$$

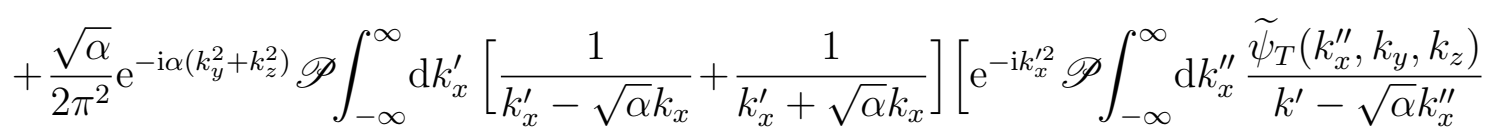

$$
\begin{aligned}
& \left.-\mathscr{P} \int_{-\infty}^{\infty} \mathrm{d} k_{x}^{\prime \prime} \frac{\widetilde{\psi}_{T}\left(k_{x}^{\prime \prime}, k_{y}, k_{z}\right)}{k_{x}^{\prime}-\sqrt{\alpha} k_{x}^{\prime \prime}} \mathrm{e}^{-\mathrm{i} \alpha k_{x}^{\prime \prime 2}}\right],
\end{aligned}
$$

in complete analogy to the one-dimensional model. In the calculation of the norm of the reflected part of the wave packet

$$
N_{\text {refl. }}(\alpha)=\int_{-\infty}^{0} \mathrm{~d} k_{x} \int_{-\infty}^{\infty} \mathrm{d} k_{y} \int_{-\infty}^{\infty} \mathrm{d} k_{z}\left|\widetilde{\psi}_{T+\tau}(\boldsymbol{k})\right|^{2}
$$

the exponential with respect to $k_{y}$ and $k_{z}$ drops out and the expansion in $\alpha$ is identical to the one-dimensional case. The first order term in the expansion gives

$$
N_{\text {refl. }}(\alpha)=2 \sqrt{\frac{2 \alpha}{\pi}} \int_{-\infty}^{\infty} \mathrm{d} y \int_{-\infty}^{\infty} \mathrm{d} z\left|\psi_{T}(0, y, z)\right|^{2} .
$$

In the three-dimensional case one therefore obtains by this experimental configuration the averaged position space density of the wave function in the plane $x=0$, as long as the mirror is switched on for a short enough time span. By rotating the plane of the mirror around the origin $\boldsymbol{x}_{0}=0$, or mathematically equivalent by letting the wave packet impinge from different directions, one can also measure the atom's density averaged over different planes defined by the mirror surface. One may also vary the point $\boldsymbol{x}_{0}$ around which the mirror is rotated. In general, one may parametrize all possible planes in $\mathbb{R}^{3}$ by the pair $(\boldsymbol{\xi}, p)$ with a unit vector $\boldsymbol{\xi}$ and a real number $p$. Then each plane $(\boldsymbol{\xi}, p)$ can be characterized by all $\boldsymbol{x}_{0}$ which satisfy $\boldsymbol{x}_{0} \cdot \boldsymbol{\xi}=p$ and the measured quantity is proportional to

$$
\check{f}(\boldsymbol{\xi}, p)=\int_{\mathbb{R}^{3}} \mathrm{~d}^{3} \boldsymbol{x}_{0} f\left(\boldsymbol{x}_{0}\right)\left|\psi\left(\boldsymbol{x}_{0}\right)\right|^{2} \delta\left(p-\boldsymbol{x}_{0} \cdot \boldsymbol{\xi}\right),
$$

with $f\left(\boldsymbol{x}_{0}\right)=\left|\psi\left(\boldsymbol{x}_{0}\right)\right|^{2}$. From the mathematical point of view this is a so called Radon transform [107]. It is the basis for many applications, as for example in medical imaging technology like computer tomography or nuclear magnetic resonance, 
in which one is interested in reconstructing information about an object which is obtained by profiles on lines or planes through this object. For sufficiently fast decreasing $C^{\infty}$-functions $f$ there exists the following inversion formula

$$
f\left(\boldsymbol{x}_{0}\right)=-\frac{1}{8 \pi^{2}} \Delta_{\boldsymbol{x}_{0}} \int_{|\boldsymbol{\xi}|=1} \mathrm{~d}^{3} \boldsymbol{\xi} \check{f}\left(\boldsymbol{\xi}, \boldsymbol{x}_{0} \cdot \boldsymbol{\xi}\right) .
$$

This means that in order to obtain the value of $|\psi|^{2}$ at the position $\boldsymbol{x}_{0}$ one in principal has to measure the quantity of Equation (5.78) for all planes going through $\boldsymbol{x}_{0}$. Indeed, it can be shown that for an exact reconstruction of $f\left(\boldsymbol{x}_{0}\right)$ one needs an infinite number of planes [107]. However, due to the large number of applications of the Radon transform, many different algorithms have been developed for inversion for practical purposes. For an overview over these methods, see for example References $[108,109]$.

Therefore, in the situation given here, by repeating the measurement for a suitable number of mirror positions, one can reconstruct the density of the wave packet. In analogy to the other applications of the Radon transform one may call this a tomography of the wave function's density. This has to be clearly distinguished from the notion of quantum state tomography in which a tomographic reconstruction of the Wigner function of a given quantum state is calculated [53].

Note that similar situation is at hand in the three dimensional extension of the operational time-of-arrival model mentioned in Chapter 4.3.2 [110]. 


\section{Chapter 6}

\section{Conclusion}

The control and manipulation of the translational and internal degrees of freedom of atoms by atom-laser interaction play a major role in modern quantum optical experiments. In this context, two distinct physical systems have been investigated in this work.

In the first part of the thesis, the influence of the dipole-dipole interaction on the fluorescence of atoms or ions in a trap was studied. Single trapped ions that are subjected to laser radiation may exhibit macroscopic light and dark periods under certain conditions. The constant rate of spontaneously emitted photons vanishes for a certain period of time when the electron is essentially shelved in a meta-stable level. For two or three such ions, three or four periods of different intensity can be seen, respectively. The dipole-dipole interaction may lead to change in the statistics of these intensity periods. Such cooperative effects were found in an experiment with two and three $\mathrm{Ba}^{+}$ions [19]. Namely, a largely increased double jump rate was measured. Double jumps occur if two consecutive upward or downward jumps happen within a period of time which is too short to be resolved experimentally. In experiments with $\mathrm{Hg}^{+}$ions such an effect was not observed [23]. More recently, again contradictory results were reported in experiments with $\mathrm{Ca}^{+}$ions $[24,25,26]$.

The goal of this part of the work was to find a theoretical description of the named experiments, especially those with $\mathrm{Ba}^{+}$and $\mathrm{Hg}^{+}$, to obtain quantitative expressions for the rate of double and triple jumps and to ascertain which of the experiments agreed with the theoretical analysis. Since the statistics of the different intensity periods is governed by the transition rates between these periods, this amounts to calculating the transition rates for level systems which reflect the main features of the effective level systems of the ions used in the experiments.

The starting point of the present work is an approach based on Bloch equations developed by Addicks et al. [17] which they used in order to calculate the transition rates for two dipole-interacting three-level systems in $\mathrm{V}$ configuration. For this system cooperative effects of up to $30 \%$ for inter-atomic distances of the order of one wave length of the strong transition were found. In this method, the level system is divided into three subsystems which can be connected to the different intensity 
periods. For each period the system is assumed to be in the corresponding subsystem. The transition rates are obtained by calculating the build-up of population in the other subsystems using a perturbation theoretical approach based on the Bloch equations.

The $\mathrm{V}$ configuration is not appropriate for the description of the ions in question

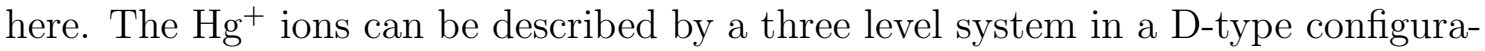
tion in which the meta-stable state is populated via spontaneous decay. The level structure of $\mathrm{Ba}^{+}$is more complicated. Therefore a four-level system was introduced in this work which contains all important features of the original level scheme.

In a first step, the Bloch equation approach of reference [17] was expanded in order to yield a treatment of two dipole-interacting D systems and two four-level systems, respectively. In contrast to the $\mathrm{V}$ system, only the transition rate from double to single intensity was found to be affected by the dipole-dipole interaction in both cases. This can be explained by the mechanisms leading to the transition between the different periods. This mechanism is incoherent in both the $\mathrm{D}$ system and the four-level system. In the case of two D systems, cooperative effects of up to $30 \%$, as for the V system, were found. However, contrary to the V system, large effects occur only for weak driving. In particular the effects are negligible for the experimental parameter values given for the $\mathrm{Hg}^{+}$ions [23], in agreement with the experimental results. In addition it was shown that the approach used in this work is more general than a theoretical treatment of two D systems in reference [37] and includes the results given there.

The cooperative effects in two four-level systems were found to be much smaller than for the $\mathrm{D}$ systems. The transition rate $p_{21}$ only differs about $1 \%$ from the value for independent atoms at an inter-atomic distance of one wave length of the laser-driven transition for the experimental values for $\mathrm{Ba}^{+}$and a value of the Rabi frequency optimized for large effects. The effect is much smaller at experimental distances of about 10 wave lengths. The effects found for the double jump rate are smaller by a factor of ten.

From this result, one may already conclude that the effects reported in reference for $\mathrm{Ba}^{+}$are not likely to be explainable by the dipole-dipole interaction. However, experimental data was only given for the case of three ions in the trap. Therefore, also the case of three dipole-dipole interacting atoms was investigated in this work. The dimensionality of the problem increases dramatically when the number of atoms is increased. Thus, the treatment was first constricted to the three level systems, both in $\mathrm{V}$ and $\mathrm{D}$ configuration. In order to reduce the number of equations, group theoretical methods are used to take advantage of the symmetries of the system. After this reduction the transition rates were obtained also for this case. The basic properties described above for the case of two atoms persist in the case of three atoms. Essentially, the strength of the cooperative effects increases by a factor of two. Effects of up to 100\% were found for both three V systems and three D systems. The effects for the experimental parameter values are still negligible, however.

The treatment of three four-level system with the approach used in the previous 
calculations is difficult, due to the increased complexity. A thorough analysis of the individual steps in this approach led to a surprising simplification permitting explicit analytical results also in this case. Similarly to the three-level systems, an enhancement of cooperative effects by a factor of two was observed. The effects for experimental parameter values are still negligible, though. It thus seems that the dipole-dipole interaction can be ruled out as an explanation of the effects observed in reference [19].

In conclusion, the results of this part of the work show that the magnitude of cooperative effects in the jump statistics of dipole-interacting atoms strongly depends on the specific level scheme, on the choice of the optical parameters (for example weak or strong driving), and also on the number of levels involved. Regarding the experiments which were investigated here, it was shown that the cooperative effects are too small to account for any increase in the double jump rate. However, with the right choice of the named parameters quite large effects may be observed. The methods presented here may also be used for the description of other level schemes, as the basic population mechanisms (coherent and incoherent driving, spontaneous emission) are incorporated in the different approaches.

In the second part of this thesis, a measurement scheme was investigated which allows to gain information about the quantum state of the translational degrees of freedom of a laser cooled atom. The idea to this scheme was taken from an experiment in which an evanescent wave atomic mirror was used to generate a temporal diffraction pattern in an atomic wave packet and most importantly to measure it by means of this mirror.

In the model presented here, the diffraction part of the experiment was merely seen as a means to prepare a wave packet that is sufficiently broad in order to be resolved by the mirror. Our investigations were focused on the detailed analysis of the measurement process. In the experiment, this process consists of switching on the mirror for a short period of time and recording the fraction of reflected atoms via the number of spontaneously emitted photons induced by a probe laser. In this work, this is modeled as follows: a wave packet with only positive momenta is prepared at some point on the negative $x$-axis. This wave packet then travels freely in the direction of positive values of $x$. At some point in time the mirror is switched on. This is done by applying reflection boundary conditions for the left and the right half axis at $x=0$, which can be seen as limiting case of a thin and steep rectangular potential barrier at this point. After a time $\tau$ the mirror is switched off again and the wave packet evolves freely, again. The norm of the reflected part of the wave packet is calculated.

The question of how the distribution obtained in this way is connected to the original wave packet for small switch-on times $\tau$ was then investigated by giving a series expansion of the reflected norm in terms of $\tau$. The lowest order of this expansion was shown to be of the order of $\sqrt{\tau}$ and proportional to the probability density of the wave function at $x=0$ and switch-on time. These findings were validated by numerical investigations. 
This result allows for two different measurement schemes which were further investigated numerically. The first scheme consists of repeating the experiment with the mirror moved to different positions and the switch-on time kept fixed. This amounts to a sampling of the wave function squared and yields the probability density of the wave function $\left|\psi\left(x, T_{0}\right)\right|^{2}$ at switch-on time $T_{0}$. This is an interesting result in itself since it is technically very difficult to resolve this quantity. Furthermore, it can be seen as a step towards quantum state reconstruction of the translational degrees of freedom of ultra-cold atoms. Such a reconstruction, in which one tries to infer the complete knowledge about a quantum state from a series of measurements, has so far only been realized for supersonic atom beams with interferometric methods $[60,62]$. The method given here is a more direct measurement. However, the information about the phase of the wave packet is lost. It might be interesting to look for schemes that also allow for the measurement of the phase using the atomic mirror.

The numerical results for this case confirmed that it is indeed possible to measure the probability density of the wave packet with good accuracy for experimentally feasible parameter values.

In the second scheme, the position of the mirror was kept fixed and the switchon time was shifted in time. This resulted in a distribution in time which if the experiment is treated classically was shown be the classical arrival-time distribution. The question of how to define a quantum mechanical arrival-time distribution is more ambiguous than in the classical case and there are a number of different theoretical proposals for this, most prominently Kijowski's distribution [86] and the quantum mechanical flux. It is therefore interesting to see whether one of these quantities is obtained with the described scheme. It was found that after normalization, the recorded distribution is essentially given by $v_{0}|\psi(0, T)|^{2}$. This quantity has also been studied in the literature and can be regarded as a semi-classical distribution [94]. It is also an approximation to Kijowski's distribution for wave packets with narrow momentum distribution.

It could be shown by numerical calculations for a wave packet prepared with values taken from the experiment, that the named distribution can indeed be measured to good accuracy with reasonable switch-on times of the mirror. For such a wave packet, however, all of the arrival-time distributions mentioned above are identical. Therefore, other wave packets were studied for which this is not the case. The difference between the distribution obtained from the model and Kijowski's distribution are clearly visible for these examples.

An interesting question for further research might be if connections to other arrival-time distributions may be found by modifying the present scheme. The advantage of this scheme over others for which such connections could be established is an easier experimental practicability.

A further result of the work is the generalization of the measurement scheme to three dimensions. If the mirror is orthogonal to the $x$-axis and positioned at $x=0$ it was shown that the measured quantity is proportional to three-dimensional 
probability distribution averaged over the plane $x=0$ for small $\tau$. By rotating the mirror and additionally moving it along the $x$-axis one accordingly obtains averages of the named quantities over all possible planes. From this data one can reconstruct a tomographic image of the probability density. 


\section{Appendix A \\ Quantum jump approach for two D systems}

For two dipole-interacting $\mathrm{D}$ systems it is also possible to apply the quantum jump approach in order to calculate the transition rates between the intensity periods. This allows to calculate higher order corrections in the interaction parameters connected with the weak transition. The procedure will first be explained for a single D system which has just two types of periods, light and dark ones. From its level configuration in Figure 2.2 it is evident that the onset of a dark period is preceded by a photon from the $|3\rangle-|2\rangle$ transition, with frequency $\omega_{2}$. Hence, starting at $t_{0}=0$ in state $|1\rangle$, the probability density for the next photon to occur at time $t$ and to come from the $|3\rangle-|2\rangle$ transition is given by

$$
w_{1 \omega_{2}}(t)=A_{2}\left|\left\langle 3\left|\mathrm{e}^{-\mathrm{i} H_{\text {cond }} t / \hbar}\right| 1\right\rangle\right|^{2}
$$

since $H_{\text {cond }}$ gives the time development between photon emissions [11]. Then its time integral,

$$
P_{\omega_{2}}=\int_{0}^{\infty} \mathrm{d} t w_{1 \omega_{2}}(t)
$$

is the probability for the next emitted photon to come from the $|3\rangle-|2\rangle$ transition. Now, let the photon rate in a light period be denoted by $I_{L}$. Then, after each photon of the light period the system is reset to the ground state and thereafter, with probability $P_{\omega_{2}}$, emits a photon from the $|3\rangle-|2\rangle$ transition. Hence the transition rate from a light to a dark period is

$$
p_{10}=I_{L} P_{\omega_{2}}
$$

This can be carried over to two dipole interacting $D$ systems as follows. An emission trajectory is considered and assumed to be in a particular intensity period, of unit intensity, say. In contrast to a single D system, the reset state after a photon emission 
in this period is not always quite the same, but it is reasonable to start from $\rho_{\mathrm{ss}, 1}$ and to use

$$
\bar{\rho}_{1} \equiv\left\{\left(A_{3}+\operatorname{Re} C_{3}\right) R_{+}^{(3)} \rho_{\mathrm{ss}, 1} R_{+}^{(3)^{\dagger}}+\left(A_{3}-\operatorname{Re} C_{3}\right) R_{-}^{(3)} \rho_{\mathrm{ss}, 1} R_{-}^{(3)^{\dagger}}\right\} / \operatorname{Tr}(\cdot)
$$

as an average reset state. The transition to a double-intensity period is marked by a photon from the $|2\rangle-|1\rangle$ transition, and therefore the probability density for such a transition, starting from the above reset state, is

$$
\begin{aligned}
w_{1 \omega_{1}}(t)=\operatorname{Tr}\left\{\left(A_{1}+\operatorname{Re} C_{1}\right)\right. & R_{+}^{(1)} \mathrm{e}^{-i H_{\text {cond }} t / \hbar} \bar{\rho}_{1} \mathrm{e}^{i H_{\text {cond }}^{\dagger} t / \hbar} R_{+}^{(1)^{\dagger}} \\
& \left.+\left(A_{1}-\operatorname{Re} C_{1}\right) R_{-}^{(1)} \mathrm{e}^{-i H_{\text {cond }} t / \hbar} \bar{\rho}_{1} \mathrm{e}^{i H_{\text {cond }}^{\dagger} t / \hbar} R_{-}^{(1)^{\dagger}}\right\}
\end{aligned}
$$

Integration over $t$ gives the total transition probability, denoted by $P_{1 \omega_{1}}$. The photon rate in a period of unit intensity is that of two dipole interacting two level systems and is given by [111]

$$
I_{\mathrm{ss}}^{(2)}=2 \frac{\Omega_{3}^{2}\left[A_{3}\left(A_{3}^{2}+2 \Omega_{3}^{2}+4 \Delta_{3}^{2}\right)+\operatorname{Re} C_{3}\left(A_{3}^{2}+4 \Delta_{3}^{2}\right)\right]}{\left(A_{3}^{2}+2 \Omega_{3}^{2}+4 \Delta_{3}^{2}\right)^{2}+\left(A_{3}^{2}+4 \Delta_{3}^{2}\right)\left(\left|C_{3}\right|^{2}+2 A_{3} \operatorname{Re} C_{3}+4 \Delta_{3} \operatorname{Im} C_{3}\right)} .
$$

Thus $p_{12}$ is given by

$$
p_{12}=I_{\mathrm{ss}}^{(2)} P_{1 \omega_{1}} \text {. }
$$

In a similar way one obtains $p_{10}$ and $p_{21}$. The transition rate $p_{01}$ can be directly read off from the no-photon probability $e^{-2 A_{1} t}$. One obtains the same results as in Section 3.2 .2 when one expands in the small parameters. In the case $\lambda_{1}, \lambda_{3} \ll r \ll \lambda_{2}$ one can put $C_{1}=C_{3}=0$ and one obtains for example

$$
p_{12}=A_{1}\left(1+\frac{\operatorname{Im} C_{2}^{2}}{A_{3}^{2}+2 \Omega_{3}^{2}+4 \Delta_{3}^{2}}\right) .
$$

The term proportional to $\operatorname{Im} C_{2}^{2}$ may have a noticeable effect for $r / \lambda_{2} \ll 1$ since the imaginary parts of the coupling parameters diverge for $r \rightarrow 0$. For experimental parameter values according to Reference [23] this term is negligible, however. 


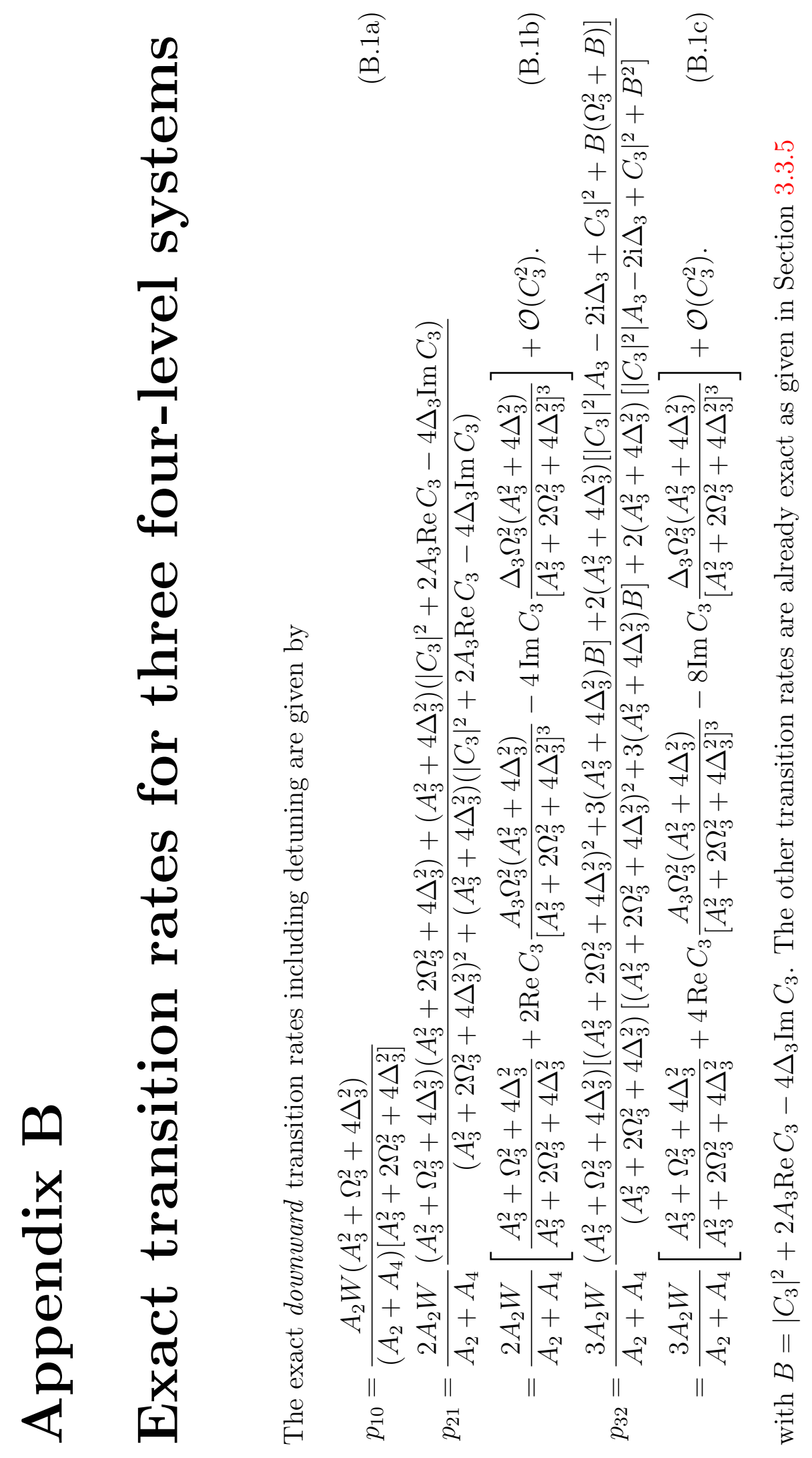




\section{Appendix C}

\section{Hilbert transforms}

Since the Hilbert transform is an integral transform which is not as commonly used as others, a few basic properties of it will be reviewed here. Furthermore the Hilbert transform for two classes of functions will be presented which are not found in the literature. For further information see the References [95, 96].

The Hilbert transform is defined by

$$
g(y)=H[f(x)]=\frac{1}{\pi} \mathscr{P} \int_{-\infty}^{\infty} \mathrm{d} x \frac{f(x)}{x-y}
$$

for real variables $x$ and $y$. In the above formula $\mathscr{P}$. denotes the Cauchy principal value. The function $f(x)$ and its Hilbert transform are skew-reciprocal, that is

$$
H[g(y)]=-f(x) .
$$

Some simple general properties of the Hilbert transform are given in table C.1. In the following a technique for the calculation of Hilbert transforms is given for the example of the function

$$
f(x)=\mathrm{e}^{-\alpha\left(x-\frac{\alpha}{\beta}\right)^{2}} \quad \alpha, \beta \in \mathbb{C}, \quad \operatorname{Re} \alpha, \operatorname{Im} \alpha \geq 0
$$

The Hilbert transform of this function is formally given by

$$
g(y)=H[f(x)]=\frac{1}{\pi} \mathscr{P} \int_{-\infty}^{\infty} \mathrm{d} x \frac{f(x)}{x-y}=\frac{1}{\pi} \mathscr{P} \int_{-\infty}^{\infty} \mathrm{d} x \frac{\mathrm{e}^{-\alpha\left(x-\frac{\alpha}{\beta}\right)^{2}}}{x-y} .
$$

With the substitution $\xi=x-y$ this can be rewritten as

$$
\begin{aligned}
g(y) & =\frac{1}{\pi} \mathscr{P} \int_{-\infty}^{\infty} \mathrm{d} x \frac{\mathrm{e}^{-\alpha\left(\xi+y-\frac{\alpha}{\beta}\right)^{2}}}{\xi}=\mathrm{e}^{-\alpha\left(y-\frac{\alpha}{\beta}\right)^{2}} \frac{1}{\pi} \mathscr{P} \int_{-\infty}^{\infty} \mathrm{d} x \frac{\mathrm{e}^{-\alpha \xi^{2}} \mathrm{e}^{-2 \alpha\left(y-\frac{\alpha}{\beta}\right) \xi}}{\xi} \\
& =\mathrm{e}^{-\alpha\left(y-\frac{\alpha}{\beta}\right)^{2}} \frac{1}{\pi} \underbrace{\mathscr{P} \int_{-\infty}^{\infty} \mathrm{d} x \frac{\mathrm{e}^{-\alpha \xi^{2}} \mathrm{e}^{-2 \alpha z \xi}}{\xi}}_{I(z)},
\end{aligned}
$$




\begin{tabular}{|c|c|c|}
\hline$f(x)$ & & $\frac{1}{\pi} \mathscr{P} \int_{-\infty}^{\infty} \mathrm{d} x \frac{f(x)}{x-y}$ \\
\hline$f(x)$ & & $g(y)$ \\
\hline$g(x)$ & & $-f(y)$ \\
\hline$f(a+x)$ & $a \in \mathbb{R}$ & $g(a+y)$ \\
\hline$f(a x)$ & $a>0$ & $g(a y)$ \\
\hline$f(-a x)$ & $a>0$ & $-g(a y)$ \\
\hline$(x+a) f(x)$ & & $(y+a) g(y)+\frac{1}{\pi} \int_{-\infty}^{\infty} \mathrm{d} x f(x)$ \\
\hline$f^{\prime}(x)$ & & $g^{\prime}(y)$ \\
\hline
\end{tabular}

Table C.1: General properties of the Hilbert transform.

where the new variable $z=y-\beta / \alpha$ has been defined. The integral which is denoted by $I(z)$ will now be evaluated as a function of $\mathrm{z}$. For $z=0$ one has

$$
I(0)=\mathscr{P} \int_{-\infty}^{\infty} \mathrm{d} \xi \frac{\mathrm{e}^{-\alpha \xi^{2}}}{\xi}=0
$$

since the integrand is an odd function. Therefore one can write

$$
I(z)=\int_{0}^{z} \mathrm{~d} t I^{\prime}(t)
$$

Evaluating the derivative of the integral gives

$$
I^{\prime}(t)=-2 \alpha \int_{-\infty}^{\infty} \mathrm{d} \xi \mathrm{e}^{-\alpha \xi^{2}} \mathrm{e}^{-2 \alpha t \xi}=-2 \alpha \mathrm{e}^{\alpha t^{2}} \int_{-\infty}^{\infty} \mathrm{d} \xi \mathrm{e}^{-\alpha(\xi+t)^{2}}=-2 \sqrt{\pi \alpha} \mathrm{e}^{\alpha t^{2}}
$$

Therefore

$$
I(z)=-2 \sqrt{\pi \alpha} \int_{0}^{z} \mathrm{~d} t \mathrm{e}^{\alpha t^{2}}
$$

and

$$
g(y)=-\mathrm{e}^{-\alpha\left(x-\frac{\alpha}{\beta}\right)^{2}} \frac{2}{\sqrt{\pi}} \int_{0}^{\sqrt{\alpha}\left(y-\frac{\beta}{\alpha}\right)} \mathrm{d} t \mathrm{e}^{t^{2}}=-\mathrm{e}^{-\alpha\left(x-\frac{\alpha}{\beta}\right)^{2}} \operatorname{erfi}\left(\sqrt{\alpha}\left(y-\frac{\beta}{\alpha}\right)\right)
$$

with the imaginary error function

$$
\operatorname{erfi}(z)=-\mathrm{i} \operatorname{erf}(\mathrm{i} z)=-\frac{2 \mathrm{i}}{\sqrt{\pi}} \int_{0}^{\mathrm{i} z} \mathrm{~d} t \mathrm{e}^{-t^{2}}=\frac{2}{\sqrt{\pi}} \int_{0}^{z} \mathrm{~d} t \mathrm{e}^{t^{2}}
$$


An analogous calculation gives the Hilbert transform $g(y)$ of the function $f(x)=$ $\mathrm{e}^{-\mathrm{i} x^{2}}$

$$
\begin{aligned}
g(y)=\frac{1}{\pi} \mathscr{P} \int_{-\infty}^{\infty} \mathrm{d} x \frac{\mathrm{e}^{-\mathrm{i} x^{2}}}{x-y} & =-\sqrt{\frac{2}{\pi}}(1+\mathrm{i}) \mathrm{e}^{-\mathrm{i} y^{2}} \int_{0}^{y} \mathrm{~d} t \mathrm{e}^{\mathrm{i} t^{2}} \\
& =-(1+\mathrm{i}) \mathrm{e}^{-\mathrm{i} y^{2}}\left(\mathrm{C}\left(\sqrt{\frac{2}{\pi}} y\right)+\mathrm{iS}\left(\sqrt{\frac{2}{\pi}} y\right)\right)
\end{aligned}
$$

where the Fresnel sine and cosine functions

$$
\mathrm{C}(x)=\int_{0}^{x} \mathrm{~d} t \cos \frac{\pi}{2} t^{2}, \quad \mathrm{~S}(x)=\int_{0}^{x} \mathrm{~d} t \sin \frac{\pi}{2} t^{2}
$$

have been used. Apart from this function also the Hilbert transforms for related functions are needed. Namely these are functions of the form

$$
f_{n}(x)=\frac{1}{x^{2 n+1}}\left[\mathrm{e}^{\mathrm{i} x^{2}}-\sum_{k=0}^{n} \frac{\left(\mathrm{i} x^{2}\right)^{k}}{k !}\right] .
$$

These can be transformed via iteration by means of the formula

$$
H[x f(x)]=y g(y)+\frac{1}{\pi} \int_{-\infty}^{\infty} \mathrm{d} x f(x)
$$

for the known transform $g(y)=H[f(x)]$. Since $H[1]=0$ this formula can be applied to $(f(x)-1) / x$ via

$$
g(y)=H[f(x)-1]=H\left[x \frac{f(x)-1}{x}\right]=y H\left[\frac{f(x)-1}{x}\right]+\frac{1}{\pi} \int_{-\infty}^{\infty} \mathrm{d} x \frac{f(x)-1}{x}
$$

and therefore

$$
H\left[\frac{f(x)-1}{x}\right]=\frac{g(y)}{y}-\frac{1}{y \pi} \int_{-\infty}^{\infty} \mathrm{d} x \frac{f(x)-1}{x} .
$$

For the odd function $f(x)=\mathrm{e}^{-\mathrm{i} x^{2}}$ one has

$$
\mathscr{P} \frac{1}{\pi} \int_{-\infty}^{\infty} \mathrm{d} x \frac{\mathrm{e}^{-\mathrm{i} x^{2}}-1}{x}=0
$$

and therefore

$$
g_{1}(y)=H\left[f_{1}(x)\right]=H\left[\frac{\mathrm{e}^{-\mathrm{i} x^{2}}-1}{x}\right]=-(1+\mathrm{i}) \mathrm{e}^{-\mathrm{i} y^{2}} \frac{\mathrm{C}\left(\sqrt{\frac{2}{\pi}} y\right)+\mathrm{iS}\left(\sqrt{\frac{2}{\pi}} y\right)}{y} .
$$

In the same way using

$$
\mathscr{P} \frac{1}{\pi} \int_{-\infty}^{\infty} \mathrm{d} x \frac{\mathrm{e}^{-\mathrm{i} x^{2}}-1}{x^{2}}=-\sqrt{2 \pi}(1+\mathrm{i})
$$


one has

$$
H\left[\frac{\mathrm{e}^{-\mathrm{i} x^{2}}-1}{x^{2}}\right]=-(1+\mathrm{i}) \frac{\mathrm{e}^{-\mathrm{i} y^{2}}\left(\mathrm{C}\left(\sqrt{\frac{2}{\pi}} y\right)+\mathrm{iS}\left(\sqrt{\frac{2}{\pi}} y\right)\right)-\sqrt{\frac{2}{\pi}} y}{y^{2}}
$$

and

$$
\begin{aligned}
g_{2}(y) & =H\left[\frac{f_{1}(x) / x+\mathrm{i}}{x}\right]=H\left[\frac{\mathrm{e}^{-\mathrm{i} x^{2}}-1+\mathrm{i} x^{2}}{x^{3}}\right] \\
& =-(1+\mathrm{i}) \frac{\mathrm{e}^{-\mathrm{i} y^{2}}\left(\mathrm{C}\left(\sqrt{\frac{2}{\pi}} y\right)+\mathrm{iS}\left(\sqrt{\frac{2}{\pi}} y\right)\right)-\sqrt{\frac{2}{\pi}} y}{y^{3}} .
\end{aligned}
$$

The process can be continued to arbitrary $n$. The next transform is given by

$$
g_{3}(y)=-(1+\mathrm{i}) \frac{\mathrm{e}^{-\mathrm{i} y^{2}}\left(\mathrm{C}\left(\sqrt{\frac{2}{\pi}} y\right)+\mathrm{iS}\left(\sqrt{\frac{2}{\pi}} y\right)\right)-\sqrt{\frac{2}{\pi}}\left(y-\mathrm{i} \frac{2}{3} y^{3}\right)}{y^{5}} .
$$




\section{Bibliography}

[1] H. G. Dehmelt. Proposed $10^{14} \Delta \nu>\nu$ laser fluorescence spectroscopy on $\mathrm{Tl}^{+}$ mono-ion oscillator II. Bull. Am Phys. Soc. 20, 60, 1975. (Cited on page 7.)

[2] W. Nagourney, J. Sandberg, and H. Dehmelt. Shelved optical electron amplifier: Observation of quantum jumps. Phys. Rev. Lett. 56, 2797, 1986. (Cited on page 7. )

[3] T. Sauter, W. Neuhauser, R. Blatt, and P. E. Toschek. Observation of quantum jumps. Phys. Rev. Lett. 57, 1696, 1986. (Cited on pages 7, 8, 25 and 58.)

[4] J. C. Bergquist, R. G. Hulet, W. M. Itano, and D. J. Wineland. Observation of quantum jumps in a single atom. Phys. Rev. Lett. 57, 1699, 1986. (Cited on pages 7 and 19.)

[5] R. J. Cook and H. J. Kimble. Possibility of direct observations of quantum jumps. Phys. Rev. Lett. 54, 1023, 1985. (Cited on page 7.)

[6] H. J. Kimble, R. J. Cook, and A. L. Wells. Intermittent atomic fluorescence. Phys. Rev. A 34, 3190, 1986. (Cited on page 7.)

[7] A. Schenzle and R. G. Brewer. Macroscopic quantum jumps in a single atom. Phys. Rev. A 34, 3127, 1986. (Cited on page 7.)

[8] J. Javanainen. Possibility of quantum jumps in a three-level system. Phys. Rev. A 33, 2121, 1986. (Cited on page 7.)

[9] C. Cohen-Tannoudu and J. Dalibard. Single-atom laser spectroscopy. looking for dark periods in fluorescence light. Europhys. Lett. 1, 441, 1986. (Cited on page 7.)

[10] P. Zoller, M. Marte, and D. F. Walls. Quantum jumps in atomic systems. Phys. Rev. A 35, 198, 1987. (Cited on page 7.)

[11] G. C. Hegerfeldt and T. S. Wilser. In H. D. Doebner, W. Scherer, and F. Schroeck, editors, Proceedings of the Second International Wigner Symposium, Singapore, 1992. World Scientific. (Cited on pages 7, 10 and 112.) 
[12] T. WILSER. Ein quantenmechanisches Modell der makroskopischen Dunkelphasen eines Laser-angeregten Einzelatoms. PhD thesis, Universität Göttingen, 1991. (Cited on pages 7, 10, 15, 16 and 18.)

[13] G. C. Hegerfeldt. How to reset an atom after a photon detection: Applications to photon-counting processes. Phys. Rev. A 47, 449, 1993. (Cited on pages $7,10,12$ and 13.)

[14] G. C. Hegerfeldt and D. G. Sondermann. Conditional Hamiltonian and reset operator in the quantum jump approach. Quantum Semiclass. Opt. 8, 121, 1996. (Cited on pages 7 and 10.)

[15] H. Carmichael. An Open Systems Approach to Quantum Optics. Lecture Notes in Physics. Springer, Berlin, 1993. (Cited on pages 7 and 10.)

[16] J. Dalibard, Y. Castin, and K. Mølmer. Wave-function approach to dissipative processes in quantum optics. Phys. Rev. Lett. 68, 580, 1992. (Cited on pages 7 and 10.)

[17] S. Addicks, A. Beige, M. Dakna, and G. C. Hegerfeldt. Double jumps and transition rates for two dipole-interacting atoms. Eur. Phys. J. D 15, 393, 2001. (Cited on pages 8, 9, 21, 28, 29, 31, 107 and 108.)

[18] Z. FICEK and R. TANAŚ. Entangled states and collective nonclassical effects in two-atom systems. Phys. Rep. 372, 369, 2002. (Cited on pages 8 and 21.)

[19] T. Sauter, R. Blatt, W. Neuhauser, and P. E. ToscheK. 'Quantum jumps' observed in the fluorescence of a single ion. Opt Commun. 60, 287, 1986. (Cited on pages 8, 19, 20, 21, 25, 31, 37, 41, 42, 43, 107 and 109.)

[20] T. SAUTER. Beobachtung von Quantensprüngen in der Resonanzfluoreszenz einzelner $\mathrm{Ba}^{+}$-Ionen. PhD thesis, Universität Hamburg, 1987. (Cited on pages $8,19,20,25,31,37,41,42$ and 43.$)$

[21] T. Sauter, R. Blatt, W. Neuhauser, and P. E. Toschek. Quantum jumps in a single ion. Pysica Scripta T22, 128, 1988. (Cited on page 8.)

[22] R. C. Thompson, D. J. Bates, K. Dholakia, D. M. Segal, and D. C. WILSON. Quantum optics with trapped and laser cooled magnesium ions. Phys. Scripta 46, 285, 1992. (Cited on pages 8, 31 and 43.)

[23] W. M. Itano, J. C. Bergquist, and D. J. Wineland. Photon antibunching and sub-poissonian statistics from quantum jumps in one and two atoms. Phys. Rev. A 38, 559, 1988. (Cited on pages 8, 19, 21, 25, 31, 33, 37, 43, 52, 107, 108 and 113.) 
[24] M. Block, O. Rehm, P. Seibert, and G. Werth. $3 d^{2} D_{5 / 2}$ lifetime in laser cooled $\mathrm{Ca}^{+}$: Influence of cooling laser power. Eur. Phys. J. D 7, 461, 1999. (Cited on pages 8, 19, 21, 43 and 107.)

[25] C. J. S. Donald, D. M. Lucas, P. A. Barton, M. J. McDonnell, J. P. Stacey, D. A. Stevens, D. N. Stacey, and A. M. Steane. Search for correlation effects in linear chains of trapped $\mathrm{Ca}^{+}$ions. Europhys. Lett. 51, 388, 2000. (Cited on pages 8, 19, 21, 43 and 107.)

[26] P. Stannum, I. S. Jensen, R. G. Martinussen, D. Voigt, and M. Drewsen. Lifetime measurement of the metastable $3 d^{2} D_{5 / 2}$ state in the ${ }^{40} \mathrm{Ca}^{+}$ion using the shelving technique on a few-ion string. Phys. Rev. A 69, 032503, 2004. (Cited on pages 8, 19 and 107.)

[27] D. J. Berkeland, D. A. Raymondson, and V. M. Tassin. Tests for nonrandomness in quantum jumps. Phys. Rev. A 69, 052103, 2004. (Cited on pages $8,21,31$ and 43.)

[28] B. H. W. Hendriks and G. Nienhus. Collective quantum jumps in pairs of atoms with two or three states. J. Mod. Opt. 35, 1331, 1988. (Cited on page 8 .)

[29] G. S. Agarwal, S. V. Lawande, and R. D'Souza. Quantum jumps in a system of few trapped ions. IEEE J. Quant. Electron. 24, 1413, 1988. (Cited on page 8.)

[30] S. V. Lawande, Q. V. Lawande, and B. N. Jagatap. Role of the dipoledipole interaction in the interpretation of quantum jumps in two three-level atoms. Phys. Rev. A 40, 3434, 1989. (Cited on page 8.)

[31] C. Fu and C. Gong. Spontaneous emission by two three-level atoms. Phys. Rev. A 45, 5095, 1992. (Cited on page 8.)

[32] M. Lewenstein and J. Javanainen. Cooperative quantum jumps with two atoms. Phys. Rev. Lett. 59, 1289, 1987. (Cited on page 8.)

[33] M. LeWEnstein and J. Javanainen. Quantum jump statistics for two-atom systems. IEEE J. Quant. Electr. 42, 1403, 1988. (Cited on page 8.)

[34] C. Skornia, J. von Zanthier, G. S. Agarwal, E. Werner, and H. WALther. Monitoring the dipole-dipole interaction via quantum jumps of individual atoms. Phys. Rev. A 64, 053803, 2001. (Cited on page 8.)

[35] A. Beige and G. C. Hegerfeldt. Cooperative effects in the light and dark periods of two dipole-interacting atoms. Phys. Rev. A 59, 2385, 1999. (Cited on page 8.) 
[36] P. R. Berman. Resonant interaction between identical atoms including recoil. Phys. Rev. A 55, 4466, 1997. (Cited on pages 9, 11 and 13.)

[37] C. Skornia, J. von Zanthier, G. S. Agarwal, E. Werner, and H. WALTHER. Inhibition of cooperative quantum jumps due to fast spontaneous decay. Europhys. Lett. 56, 665, 2001. (Cited on pages 9, 37 and 108.)

[38] M. B. Plenio and P. L. Knight. The quantum-jump approach to dissipative dynamics in quantum optics. Rev. Mod. Phys. 70, 101, 1998. (Cited on page 10.)

[39] A. Beige and G. C. Hegerfeldt. Projection postulate and quantum Zeno effect. Phys. Rev. A 53, 53, 1996. (Cited on pages 11 and 17.)

[40] The projection postulate as commonly used nowadays is due to G. Lüders, Ann. Phys. 8, 323 (1951). For observables with degenerate eigenvalues his formulation differs from that of J. von Neumann, Mathematische Grundlagen der Quantenmechanik, (Springer, Berlin, 1932). (Cited on page 11.)

[41] G. S. Agarwal. Quantum Statistical Theories of Spontaneous Emission and their Relation to Other Approaches, volume 70 of Springer Tracts in Modern Physics. Springer-Verlag, Berlin, 1974. (Cited on page 13.)

[42] G. C. Hegerfeldt and M. B. Plenio. Macroscopic dark periods without a metastable state. Phys. Rev. A 46, 373, 1992. (Cited on page 16.)

[43] A. Beige, G. C. Hegerfeldt, and D. G. Sondermann. Laser pulses as measurements: application to the quantum Zeno effect. Quantum Semiclass. Opt. 8, 999, 1996. (Cited on page 17.)

[44] A. Beige. Kooperative Effekte in der Fluoreszenz zweier Atome. PhD thesis, Universität Göttingen, Januar 1997. (Cited on pages 23 and 25.)

[45] W. M. Itano, J. C. Bergquist, R. G. Hulet, and D. J. Wineland. Radiative decay rates in $\mathrm{Hg}^{+}$from observation of quantum jumps in a single ion. Phys. Rev. Lett. 59, 2732, 1987. (Cited on pages 33 and 52.)

[46] V. Hannstein and G. C. Hegerfeldt. Cooperative effects for experimentally relevant dipole-dipole interacting level systems. Phys. Rev. A 68, 043826, 2003. (Cited on pages 35 and 42.)

[47] G. C. Hegerfeldt and M. B. Plenio. Coherence with incoherent light: A new type of quantum beats for a single system. Phys. Rev. A 47, 2186, 1993. (Cited on pages 38 and 39.) 
[48] V. Hannstein and G. C. Hegerfeldt. Cooperative quantum jumps for three dipole-dipole interacting atoms. Phys. Rev. A 70, 023820, 2004. (Cited on page 58.)

[49] P. Szriftgiser, D. Guéry-Odelin, M. Arndt, and J. Dalibard. Atomic wave diffraction and interference using temporal slits. Phys. Rev. Lett. 77, 4, 1996. (Cited on pages 61, 65, 66, 67, 79 and 98.)

[50] P. SzRiftgiser. Cavité gravitationelle et optique atomique temporelle. PhD thesis, Université Paris VI, 1996. (Cited on pages 61, 64 and 66.)

[51] A. Steane, P. Szriftgiser, P. Desbiolles, and J. Dalibard. Phase modulation of atomic de broglie waves. Phys. Rev. Lett. 74, 4972, 1995. (Cited on page 61.)

[52] M. Arndt, P. Szriftgiser, J. Dalibard, and A. M. Steane. Atom optics in the time domain. Phys. Rev. A 53, 3369, 1996. (Cited on page 61.)

[53] M. PARIS and J. ŘEHÁČEK, editors. Quantum State Estimation, volume 649 of Lecture Notes in Physics. Springer, 2004. (Cited on pages 62, 95 and 106.)

[54] U. Leonhardt. Measuring the quantum state of light. Cambridge studies in modern physics. Cambridge Univ. Press, 1997. (Cited on pages 62 and 95.)

[55] D. Leibfried, D. M. Meekhof, B. E. King, C. Monroe, W. M. Itano, and D. J. WinELAND. Experimental determination of the motional quantum state of a trapped atom. Phys. Rev. Lett. 77, 4281, 1996. (Cited on pages 62 and 95.)

[56] T. J. Dunn, I. A. Walmsley, and S. Mukamel. Experimental determination of the quantum-mechanical state of a molecular vibrational mode using fluorescence tomography. Phys. Rev. Lett. 74, 884, 1995. (Cited on page 62.)

[57] C. Monroe. Quantum information processing with atoms and photons. Nature 416, 238, 2002. (Cited on pages 62 and 95.)

[58] F. Schmidt-Kaler, H. Häffner, M. Riebe, S. Gulde, G. P. T. Lancaster, T. Deuschle, C. Becher, C. F. Roos, J. Eschner, and R. BlatT. Realization of the cirac-zoller controlled-NOT quantum gate. Nature 422, 408, 2003. (Cited on pages 62 and 95.)

[59] T. Pfau and C. Kurtsiefer. Partial reconstruction of the motional wigner function of an ensemble of helium atoms. J. Mod. Opt. 44, 2551, 1997. (Cited on pages 62 and 95.) 
[60] C. Kurtsiefer, T. Pfau, and J. Mlynek. Measurement of the Wigner function of an ensemble of helium atoms. Nature 386, 150, 1997. (Cited on pages 62,95 and 110.)

[61] R. A. Rubenstein, A.-A. Dhirani, D. A. Kokorowski, T. D. Roberts, E. T. Smith, W. W. Smith, H. J. Bernstein, J. Lehner, S. Gupta, and D. E. PRITCHARD. Search for off-diagonal density matrix elements for atoms in a supersonic beam. Phys. Rev. Lett. 82, 2018, 1999. (Cited on pages 62 and 96.)

[62] R. A. Rubenstein, D. A. Kokorowski, A.-A. Dhirani, T. D. Roberts, S. Gupta, J. Lehner, W. W. Smith, E. T. Smith, H. J. Bernstein, and D. E. PRITCHARD. Measurement of the density matrix of a longitudinally modulated atomic beam. Phys. Rev. Lett. 83, 2285, 1999. (Cited on pages 62, 96 and 110.)

[63] J.G. Muga and G.R. Leavens. Arrival time in quantum mechanics. Phys. Rep. 338, 353, 2000. (Cited on pages 62, 73, 74 and 75.)

[64] J.A. Damborenea, I.L. Egusquiza, G.C. Hegerfeldt, and J.G. Muga. Measurement-based approach to quantum arrival times. Phys. Rev. A 66, 052104, 2002. (Cited on pages 62, 76 and 99.)

[65] G.C. Hegerfeldt, D. Seidel, and J.G. Muga. Quantum arrival times and operator normalization. Phys. Rev. A 68, 022111, 2003. (Cited on pages 62, 76 and 99.)

[66] C. Cohen-Tannoudji. Atomic motion in laser light. In J. Dalibard, J.M. RAIMOND, and J. ZINN-Justin, editors, fundamental systems in quantum optics, Les Houches 1990. North-Holland, 1990. (Cited on pages 65 and 68.)

[67] M. Moshinsky. Diffraction in time. Physical Review 88, 625, 1952. (Cited on page 65.)

[68] M. Moshinsky. Diffraction in time and the time-energy uncertainty principle. Am. J. Phys. 44, 1037, 1976. (Cited on page 65.)

[69] A. S. Gerasimov and M. V. Kazarnovskir. Possibility of observing nonstationary quantum-mechanical effects by means of ultracold neutrons. Sov. Phys. JETP 44, 892, 1976. (Cited on page 66.)

[70] J. Felber, G. Müller, R. Gähler, and R. Golub. Time dependent netron optics. Physica B 162, 191, 1990. (Cited on page 66.)

[71] Časlav Brukner and A. Zeilinger. Diffraction of matter waves in space in time. Phys. Rev. A 56, 3804, 1997. (Cited on page 66.) 
[72] R. J. Cook and R. K. HiLl. An electromagnetic mirror for neutral atoms. Opt. Commun. 43, 258, 1982. (Cited on page 67.)

[73] V. Balykin, V. Letokhov, Y. B. Ovchinnikov, and A. Sidorov. Reflection of an atomic beam from a gradient of an optical field. JETP Lett. 45, 282, 1987. (Cited on page 67.)

[74] V. Balykin, V. Letokhov, Y. B. Ovchinnikov, and A. Sidorov. Quantum-state-selective mirror reflection of atoms by laser light. Phys. Rev. Lett. 60, 2137, 1988. (Cited on page 67.)

[75] M. A. Kasevich, D. S. Weiss, and S. CHu. Normal-incidence reflection of slow atoms from an optical evanescent wave. Opt. Lett. 15, 607, 1990. (Cited on page 67.)

[76] J. D. JaCKson. Classical electrodynamics. Wiley, 1962. (Cited on page 67.)

[77] R. Zwanzig. Ensemble method in the theory of irreversibility. J. Chem. Phys. 33, 1338, 1960. (Cited on page 68.)

[78] H. MorI. Transport, collective motion, and brownian motion. Prog. Theor. Phys. 33, 423, 1965. (Cited on page 68.)

[79] A. Ruschhaupt, J.A. Damborenea, B. Navarro, J.G. Muga, and G.C. HeGERFELDT. Exact and approximate complex potentials for modelling time observables. Europhys. Lett. 67, 1, 2004. (Cited on page 68.)

[80] G. Hegerfeldt. The quantum jump approach and quantum trajectories. In F. Benatti and R. Floreanini, editors, Irreversible Quantum Dynamics, volume 622 of Springer LNP, page 233. Springer, 2003. (Cited on page 68.)

[81] C. Henkel, J.-Y. Courtois, R. Kaiser, and A. Aspect. Phase shifts of atomic de broglie waves at an evanescent wave mirror. Laser Physics 4, 1042, 1994. (Cited on page 71.)

[82] M. Abramowitz and I. A. Stegun, editors. Handbook of Mathematical Functions, chapter 9, pages 374-378. Dover, New York, 10th edition, 1972. (Cited on pages 71, 72 and 73.)

[83] J.G. Muga, R. Sala, I.L. Egusquiza (eds.). Time in Quantum Mechanics. Springer, Berlin, 2002. (Cited on page 73.)

[84] A.J. Bracken and G.F. Melloy. Probability backflow and a new dimensionless quantum number. J. Phys. A: Math. Gen. 27, 2197, 1994. (Cited on page 75 .) 
[85] G.R. Allcock. Ann. Phys. (N.Y.) 53, 253;286;311, 1969. (Cited on page 75.)

[86] J. KIJOwsKI. On the time operator in quantum mechanics and the heisenberg uncertainty relation for energy and time. Rep. Math. Phys. 6, 361, 1974. (Cited on pages 75 and 110.)

[87] J. KiJowski. Comment on "Arrival time in quantum mechanics" and "Time of arrival in quantum mechanics". Phys. Rev. A 59, 897, 1999. (Cited on page 75.$)$

[88] R. Giannitrapani. Int. J. Theor. Phys. 36, 1575, 1997. (Cited on page 76.)

[89] Y. Aharonov and D. Bohm. Time in the quantum theory and the uncertainty relation for time and energy. Phys. Rev. 122, 1649, 1961. (Cited on page 76.)

[90] J. Leon, J. Julve, P. Pitanga, and F.J. De Urries. Time of arrival in the presence of interaction. Phys. Rev. A 61, 062101, 2000. (Cited on page 76.)

[91] A.D. Baute, I.L. Egusquiza, and J.G. Muga. Time-of-arrival distributions for interaction potentials. Phys. Rev. A 64, 012501, 2001. (Cited on page 76. )

[92] G.C. Hegerfeldt, D. Seidel, J.G. Muga, and B. Navarro. Operatornormalized arrival times in the presence of interactions. Phys. Rev. A 70, 012110, 2004. (Cited on page 76.)

[93] J. G. Muga, D. Seidel, and G. C. Hegerfeldt. Quantum kinetic energy densities an operational approach. J. Chem. Phys. 122, 154106, 2005. (Cited on page 76.)

[94] K. Aoki, A. Horikoshi, and E. Nakamura. Time of arrival through interacting environments: Tunneling processes. Phys. Rev. A 62, 022101, 2000. (Cited on pages 76 and 110.)

[95] A. ERdÉLYI, editor. Tables of Integral Transforms, volume II, chapter XV. McGraw-Hill, 1954. (Cited on pages 81 and 115.)

[96] E. C. Titchmarsh. Introduction to the theory of Fourier Integrals, chapter V. Oxford: Clarendon Press, 2nd edition, 1967. (Cited on pages 81 and 115.)

[97] E. Granot and A. Marchewka. Generic short-time propagation of sharpboundaries wave packets. Europhys. Lett. 72, 341, 2005. (Cited on page 89.) 
[98] R. Brühl, P. Fouquet, R. E. Grisenti, J. P. Toennies, G. C. Hegerfeldt, T. Köhler, M. Stoll, and C. Walter. The van-der-Waals potential between meta-stable atoms and solid surfaces: Novel diffraction experiments vs. theory. Europhys. Lett. 59, 357, 2002. (Cited on page 95.)

[99] M. Baron, H. RAuCh, and M. SudA. First attempt of neutron quantum state reconstruction. J. Opt. B: Quantum Semiclass. Opt. 5, S241, 2003. (Cited on page 96.)

[100] D. A. Kokorowski and D. E. Pritchard. Longitudinal quantum beam tomography. J. Mod. Opt. 44, 2575, 1997. (Cited on page 96.)

[101] A.-A. Dhirani, D. A. Kokorowski, R. A. Rubenstein, T. D. Hammond, B. Rohwedder, E. T. Smith, A. D. Roberts, and D. E. PRITCHARD. Determining the density matrix of a molecular beam using a longitudinal matter wave interferometer. J. Mod. Opt. 44, 2583, 1997. (Cited on page 96.)

[102] D. E. Pritchard, R. A. Rubenstein, A.-A. Dhirani, D. A. Kokorowski, E. T. Smith, T. D. Hammond, and B. Rohwedder. Longitudinal atom optics using localized oscillating fields: A fully quantum-mechanical treatment. Phys. Rev. A 59, 4641, 1999. (Cited on page 96.)

[103] D. A. Kokorowski, T. D. Roberts, R. A. Rubenstein, E. T. Smith, and D. E. PRITChARD. Longitudinal atom interferometry. Fortschr. Phys. 48, 615, 2000. (Cited on page 96.)

[104] K. T. Kapale, S. QAmar, and M. S. Zubairy. Spectroscopic measurement of an atomic wave function. Phys. Rev. A 67, 023805, 2003. (Cited on page 96.)

[105] B.-G. Englert, J. Schwinger, A. O. Barut, and M. O. Scully. Reflecting slow atoms from a micromaser field. Europhys. Lett. 14, 25, 1991. (Cited on page 96.)

[106] A. Del CAmpo and J. G. Muga. Single-particle matter wave pulses. J. Phys. A: Math. Gen. 38, 9803, 2005. (Cited on page 103.)

[107] S. Helgason. The Radon transform. Progress in Mathematics. Birkhäuser, 2nd edition, 1999. (Cited on pages 105 and 106.)

[108] S. R. Deans. The Radon Transform and some of its Applications. Krieger Publishing Company, 1993. (Cited on page 106.)

[109] G. T. HeRman, editor. Image reconstruction from projections: implementation and applications, volume 32 of Topics in applied physics. Springer, 1979. (Cited on page 106.) 
[110] V. Hannstein, G. C. Hegerfeldt, and J. G. Muga. Quantum optical time-of-arrival model in three dimensions. J. Phys. B: At. Mol. Opt. Phys. 38, 409, 2005. (Cited on page 106.)

[111] A. Beige and G. C. Hegerfeldt. Transition from antibunching to bunching for two dipole-interacting atoms. Phys. Rev. A 58, 4133, 1998. (Cited on page 113.) 


\section{Danksagung}

An dieser Stelle möchte ich einer Reihe von Personen danken, die in der ein oder anderen Weise dazu beigetragen haben, dass diese Arbeit zustande kommen konnte.

Zuallererst danke ich Herrn Prof. Dr. Gerhard C. Hegerfeldt für die erstklassige Betreuung während der Zeit der Promotion und für die interessante Themenstellung. Herrn Prof. Dr. Kurt Schönhammer danke ich für die Übernahme des Korreferats.

Desweiteren möchte ich Dirk Seidel für die angenehme Zeit im gemeinsamen Büro danken. Ihm und Jens Timo Neumann gilt mein Dank für die vielen hilfreichen Diskussionen im Zusammenhang mit der Arbeit und noch viel mehr für das Korrekturlesen dieser Arbeit. Meinen Eltern danke ich für die Unterstützung während der Promotionszeit.

Inga danke ich dafür, dass sie immer für mich da war und ist.

Finanziell unterstützt wurde diese Arbeit durch ein Georg-Christoph-LichtenbergStipendium des Landes Niedersachsen im Rahmen der Göttingen Graduate School of Physics.

\section{Acknowledgements}

Furthermore, I would like to thank Prof. J. Gonzalo Muga from the University of the Basque Country, Bilbao, for many fruitful discussions which often led to new ideas or solutions. I would also like to thank the people in his group, especially Beatriz Navarro, Fernando Delgado, and Andreas Ruschhaupt, for the good cooperation. I am greatful to Iñigo Egusquiza, Rafael Sala Mayato, José P. Palao, and all the other people from Bilbao named above for the nice time I had during the workshops in Bilbao and Tenerife. 



\section{Lebenslauf}

Volker Martin Hannstein

Nonnenstieg 39

37075 Göttingen

\section{Biographische Daten}

Geburtsdatum: 22. Oktober 1975

Geburtsort: $\quad$ Freiburg im Breisgau

Staatsangehörigkeit: deutsch

\section{Wissenschaftlicher Werdegang}

$07 / 1995$

$1995-1996$

$1996-1999$

$09 / 1998$

$1999-2002$

$05 / 2002$

$06 / 2002-06 / 2003$

ab $07 / 2003$

$08 / 2002-03 / 2006$
Abitur am Ganztagsgymnasium Barsinghausen

Zivildienst beim Blindenverband Niedersachsen in Hannover

Studium der Physik an der Technischen Universität Carolo-Wilhelmina zu Braunschweig

Vordiplom in Physik

Studium der Physik an der Georg-August-Universität Göttingen, Diplomarbeit am Institut für Theoretische Physik unter Anleitung von Prof. Dr. G.C. Hegerfeldt

Diplom in Physik

wissenschaftliche Hilfskraft am Institut für Theoretische Physik

Georg-Christoph-Lichtenberg-Stipendium des Landes Niedersachsen

Promotion am Institut für Theoretische Physik unter Anleitung von Prof. Dr. G.C. Hegerfeldt 